Aus der Klinik für Dermatologie, Venerologie und Allergologie Fachbereich klinische Medizin der Medizinischen Fakultät (Direktor: Univ.-Prof. Dr. med. T. Vogt) Universität des Saarlandes, Homburg/Saar

\title{
Evaluation des Gesundheitsverhaltens und der Lebensqualität dermatoonkologischer Patienten nach dermatochirurgischen Interventionen
}

Dissertation zur Erlangung des Grades eines Doktors der Medizin der Medizinischen Fakultät der UNIVERSITÄT DES SAARLANDES

2018

vorgelegt von

Detlef Peter Werner Hoernecke

geboren am 28.12.1983 in Ludwigsburg 



\section{INHALTSVERZEICHNIS}

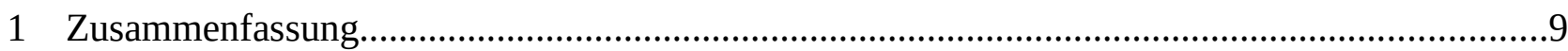

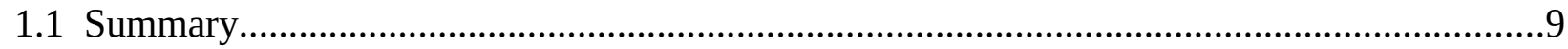

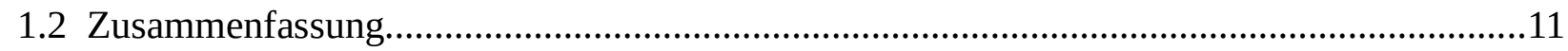

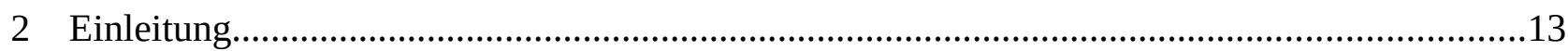

2.1 Ziele und Fragestellungen dieser Arbeit.....................................................................13

2.2 Einteilung und Epidemiologie von Hauttumoren............................................................13

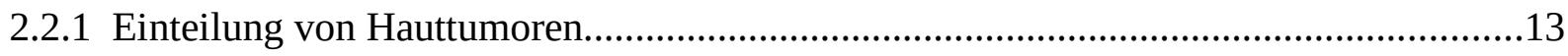

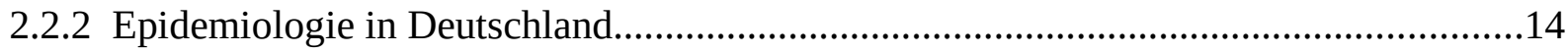

2.2.2.1 Krebserkrankungen im Allgemeinen....................................................................14

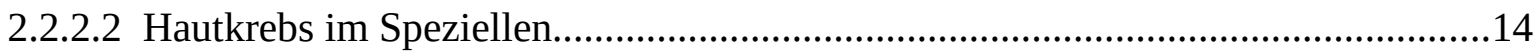

2.3 Wichtige Risikofaktoren für die Entwicklung eines Hauttumors.......................................16

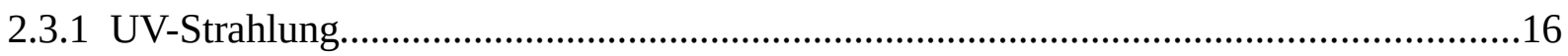

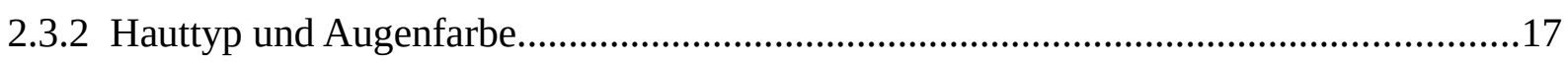

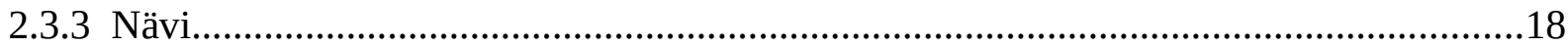

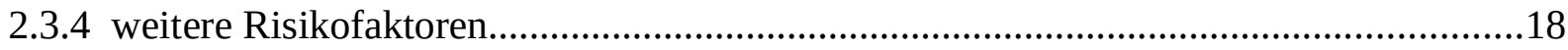

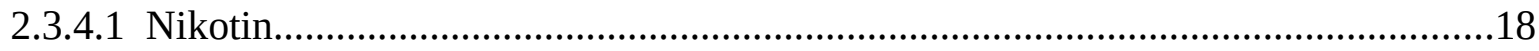

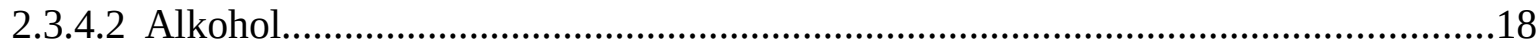

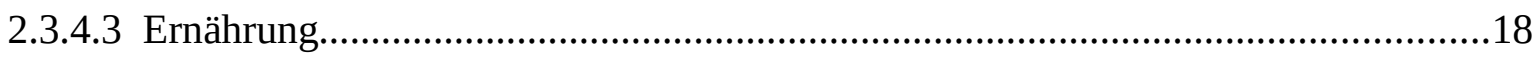

2.4 Prävention von Hautkrebserkrankungen - mögliche Ansatzpunkte....................................19

2.5 Lifestyle changes nach Tumorerkrankungen.................................................................20

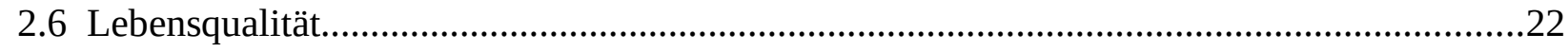

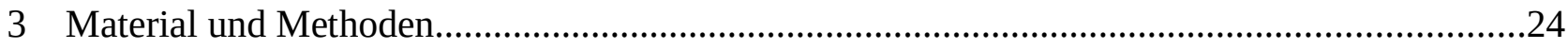

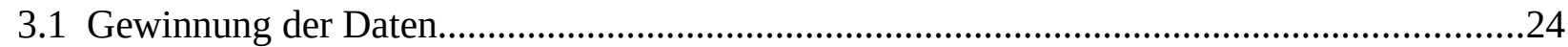

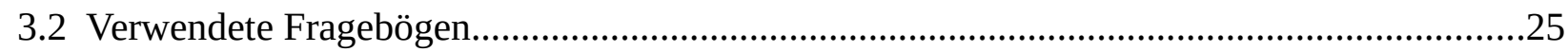

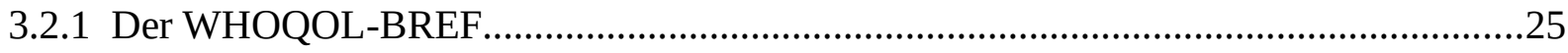

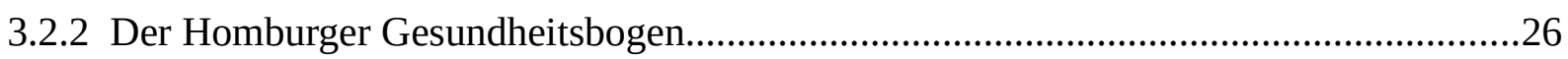

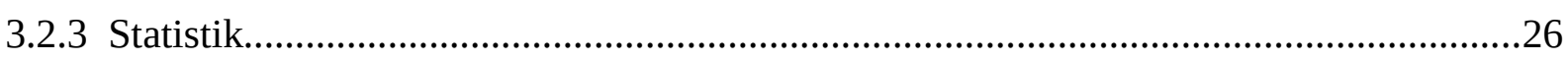

3.2.3.1 Statistische Auswertung des WHOQOL-BREFs..................................................27

3.2.3.2 Statistische Auswertung des Homburger Gesundheitsbogens................................28

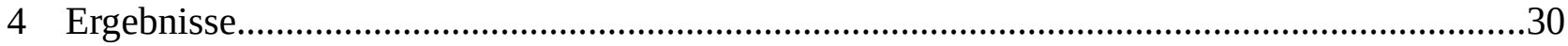

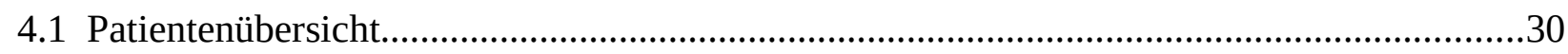




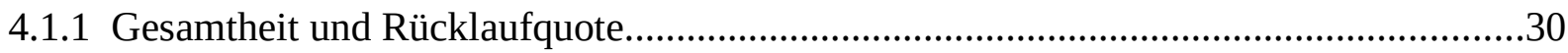

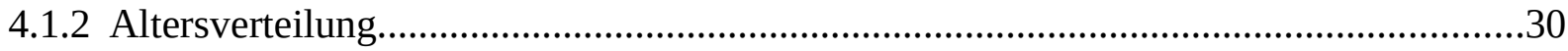

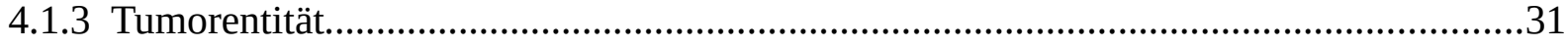

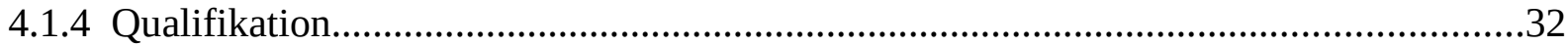

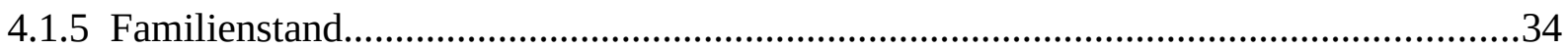

4.2 Ergebnisse des WHOQOL-BREF Fragebogens.........................................................36

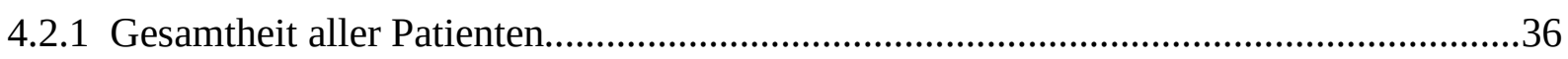

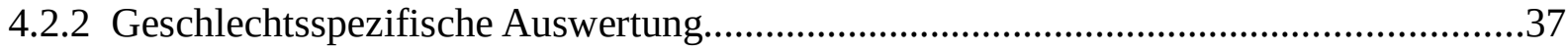

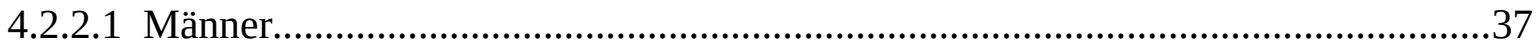

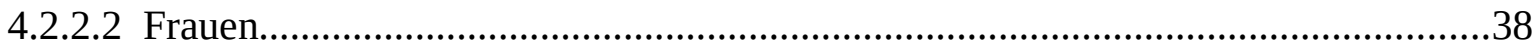

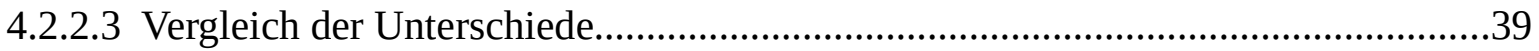

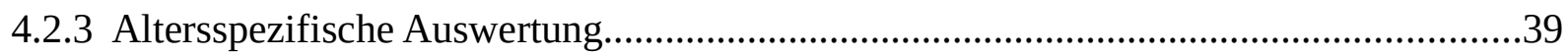

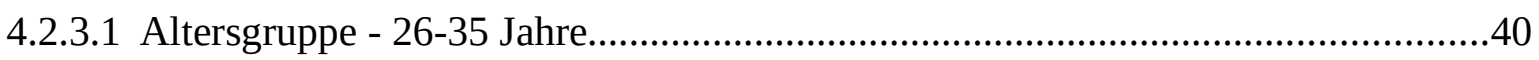

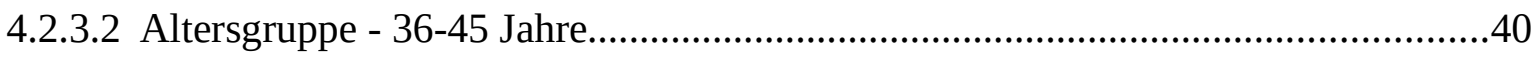

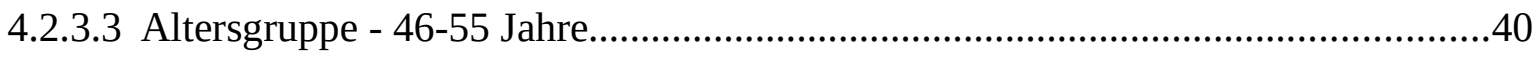

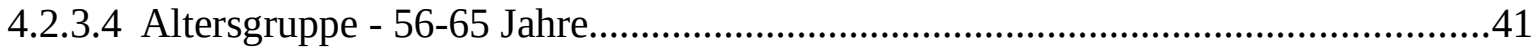

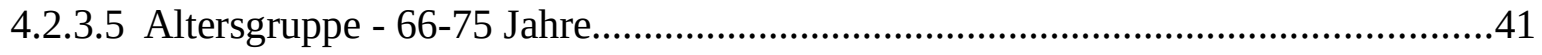

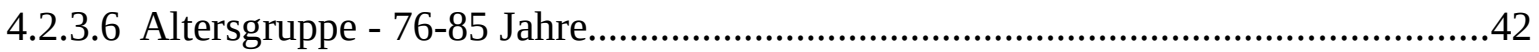

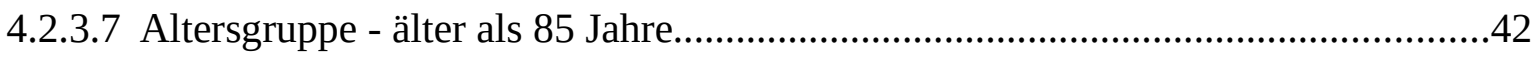

4.2.3.8 Vergleich der verschiedenen Altersgruppen......................................................42

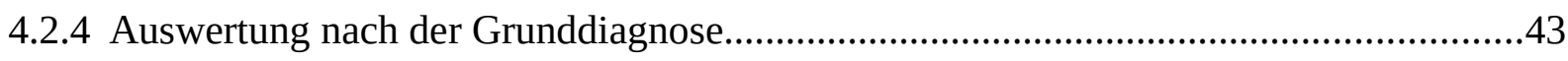

4.2.4.1 Patienten mit melanozytärem Tumor......................................................................43

4.2.4.2 Patienten mit nicht melanozytärem Tumor..........................................................44

4.2.4.3 Patienten mit beiden Tumorentitäten..................................................................44

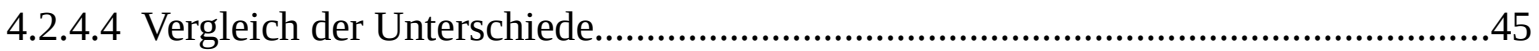

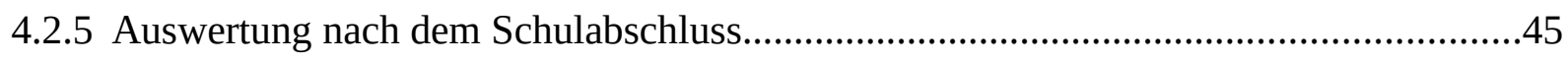

4.2.5.1 Qualifikation: kein Abschluss, Hauptschule, mittlere Reife.................................45

4.2.5.2 Qualifikation: Fachhochschulreife, Abitur, Fachhochschule, Universität,

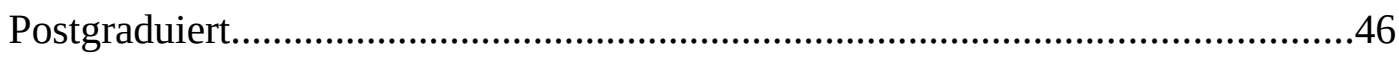

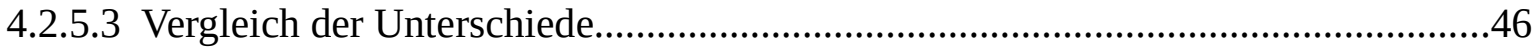

4.2.6 Auswertung nach dem Familienstand.......................................................................47

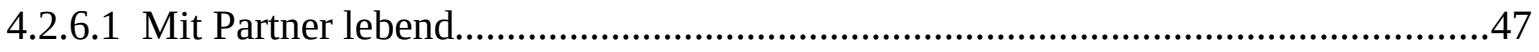




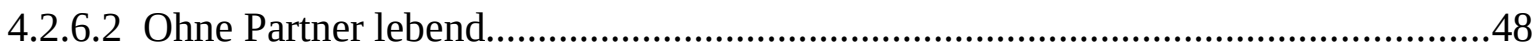

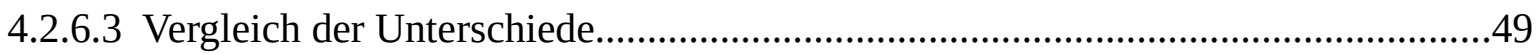

4.3 Ergebnisse des Homburger Gesundheitsbogens...............................................................51

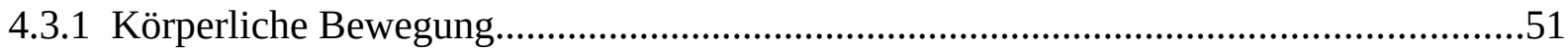

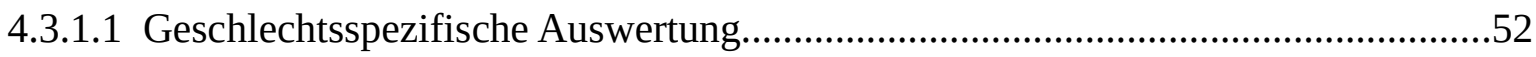

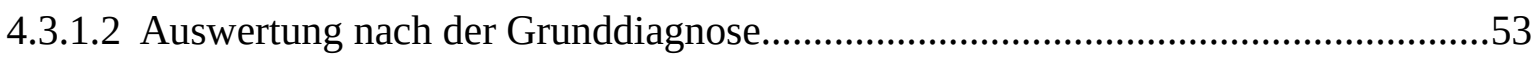

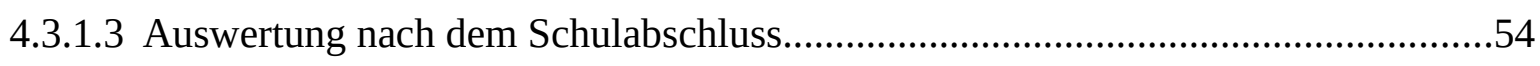

4.3.1.4 Auswertung nach dem Familienstand....................................................................55

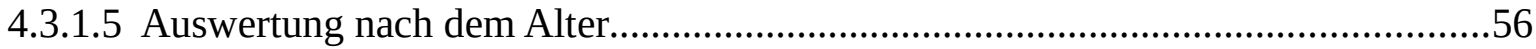

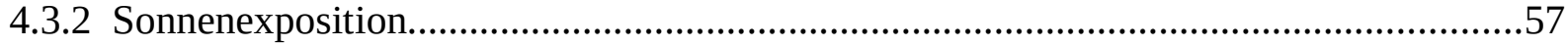

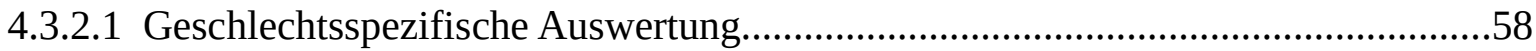

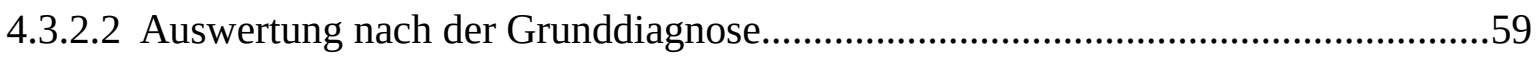

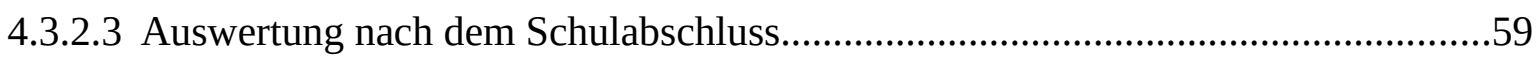

4.3.2.4 Auswertung nach dem Familienstand..................................................................60

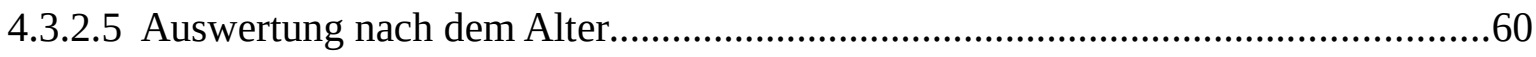

4.3.3 Verwendung von textilen und topischen UV-Schutzmöglichkeiten.............................61

4.3.3.1 Geschlechtsspezifische Auswertung.....................................................................62

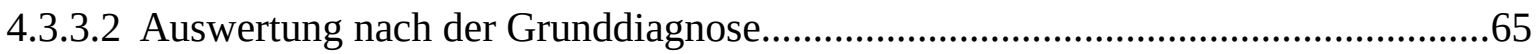

4.3.3.3 Auswertung nach dem Schulabschluss...........................................................66

4.3.3.4 Auswertung nach dem Familienstand..............................................................68

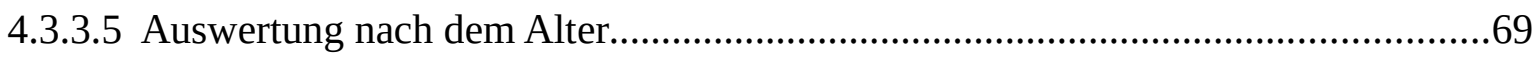

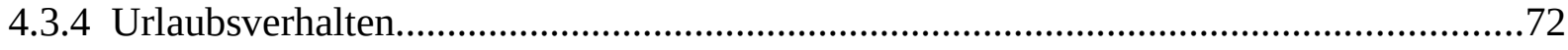

4.3.4.1 Geschlechtsspezifische Auswertung.....................................................................72

4.3.4.2 Auswertung nach der Grunddiagnose..................................................................

4.3.4.3 Auswertung nach dem Schulabschluss...............................................................73

4.3.4.4 Auswertung nach dem Familienstand...............................................................74

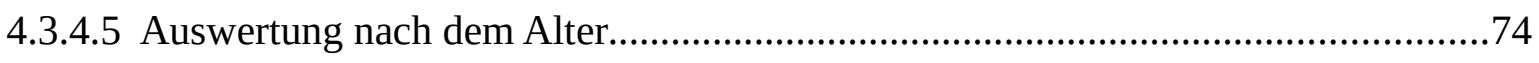

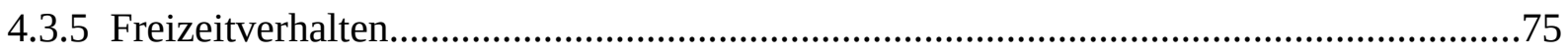

4.3.5.1 Geschlechtsspezifische Auswertung....................................................................76

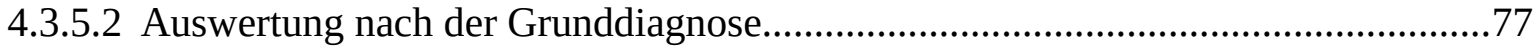

4.3.5.3 Auswertung nach dem Schulabschluss................................................................78

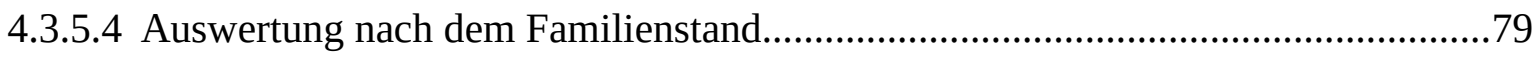




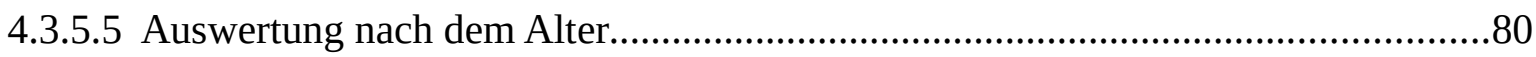

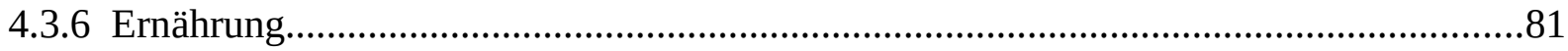

4.3.6.1 Geschlechtsspezifische Auswertung....................................................................82

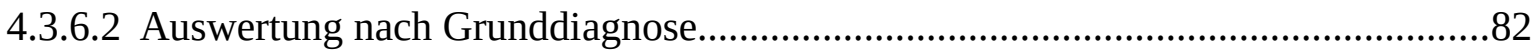

4.3.6.3 Auswertung nach dem Schulabschluss................................................................83

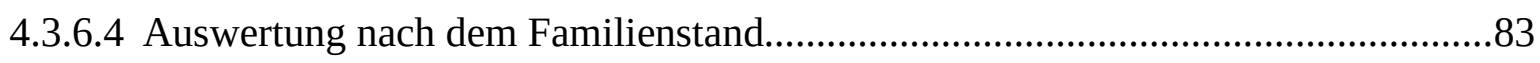

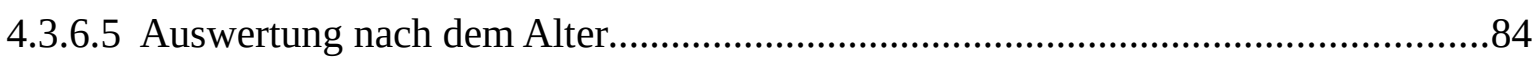

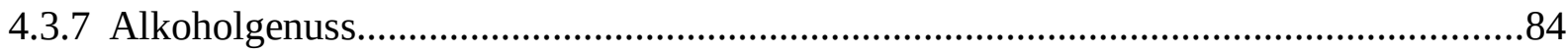

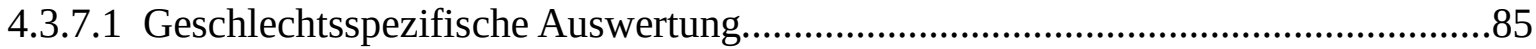

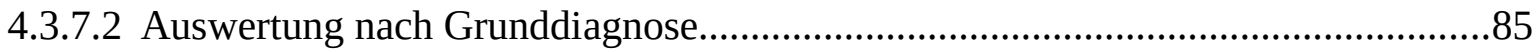

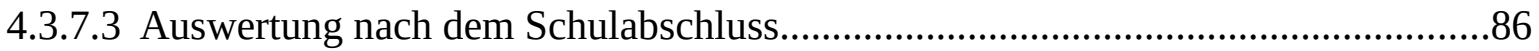

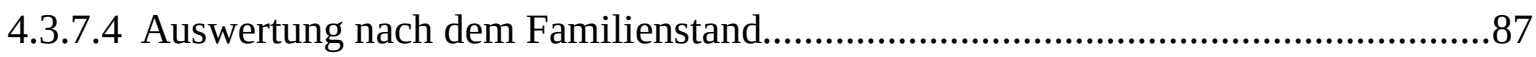

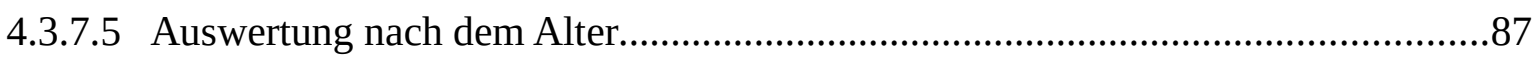

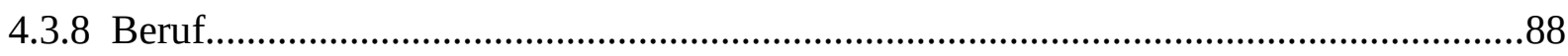

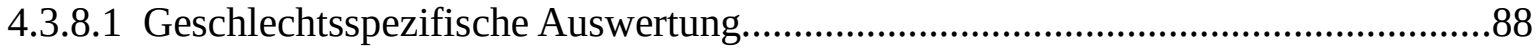

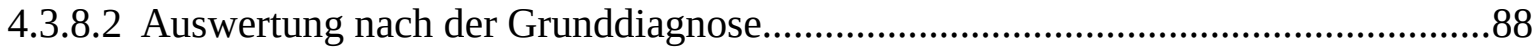

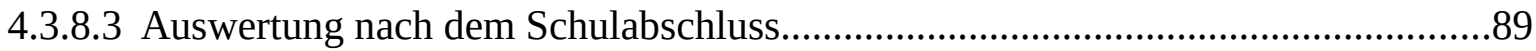

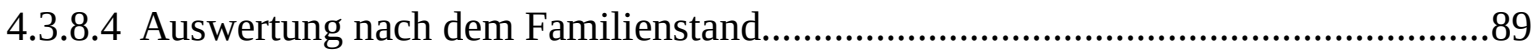

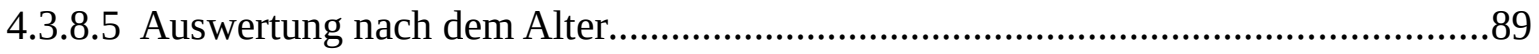

4.3.9 Wahrnehmung der Hautkrebsvorsorgeuntersuchungen..............................................90

4.3.9.1 Geschlechtsspezifische Unterschiede...................................................................91

4.3.9.2 Auswertung nach Grunddiagnose........................................................................93

4.3.9.3 Auswertung nach dem Schulabschluss..............................................................94

4.3.9.4 Auswertung nach dem Familienstand...................................................................95

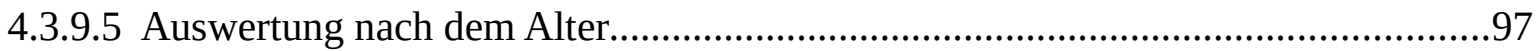

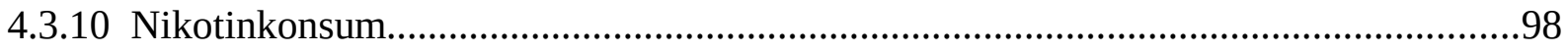

4.3.10.1 Geschlechtsspezifische Auswertung..............................................................99

4.3.10.2 Auswertung nach der Grunddiagnose................................................................99

4.3.10.3 Auswertung nach dem Schulabschluss..............................................................100

4.3.10.4 Auswertung nach dem Familienstand................................................................100

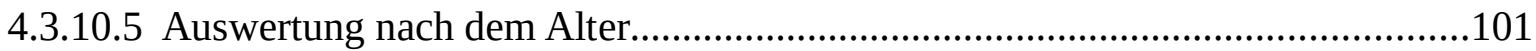




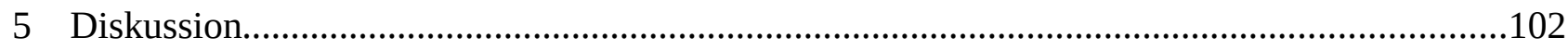

5.1 Allgemeine Limitationen............................................................................................102

5.2 Patientenübersicht und häufigste Antworten......................................................................103

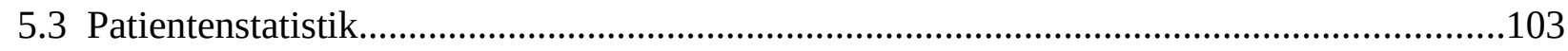

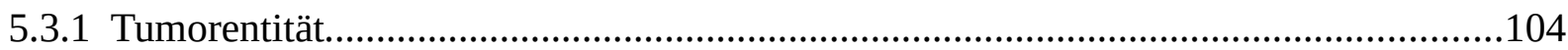

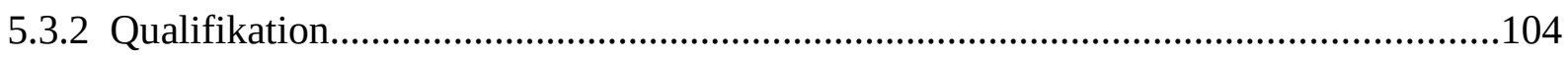

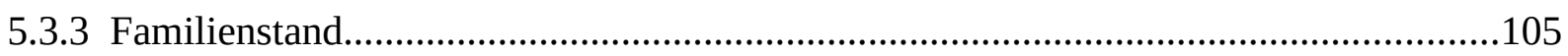

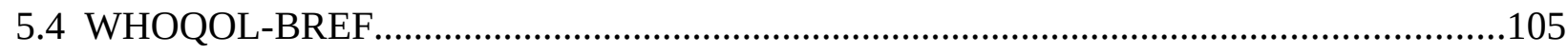

5.4.1 Geschlechtsspezifische Betrachtung..........................................................................108

5.4.2 Familienstand und Qualifikation...............................................................................109

5.4.3 Tumorentität.................................................................................................

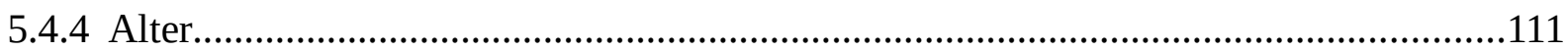

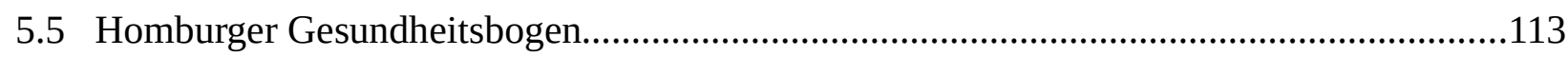

5.5.1 Allgemeine Limitationen des Homburger Gesundheitsbogens......................................113

5.5.2 Körperliche Bewegung..............................................................................................113

5.5.2.1 Vergleich der Subgruppen.................................................................................114

5.5.3 Sonnenexposition, Sonnenschutz und Freizeitverhalten bzw. Urlaubsverhalten...........115

5.5.3.1 Geschlechtsspezifische Betrachtung....................................................................117

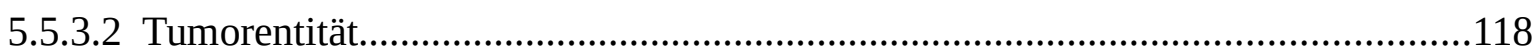

5.5.3.3 Qualifikation................................................................................................119

5.5.3.4 Familienstand.................................................................................................119

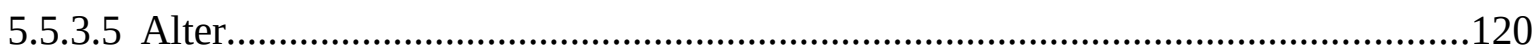

5.5.4 Hautkrebsvorsorgeuntersuchung...........................................................................120

5.5.4.1 Vergleich der Subgruppen................................................................................122

5.5.5 Ennährung und Alkoholkonsum................................................................................123

5.5.5.1 Vergleich der Subgruppen...............................................................................124

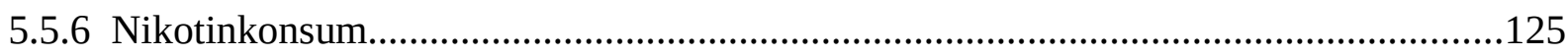

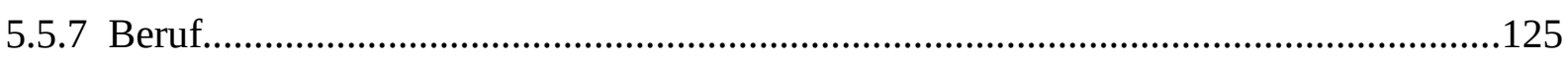

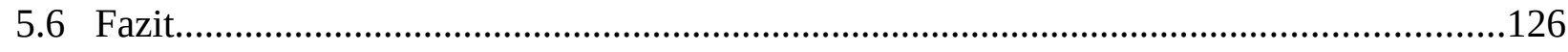

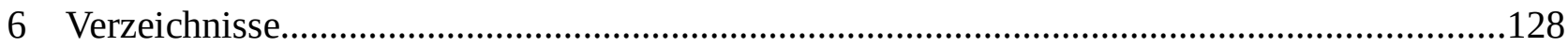

6.1 Abkürzungsverzeichnis..............................................................................................128

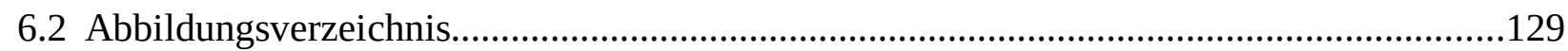




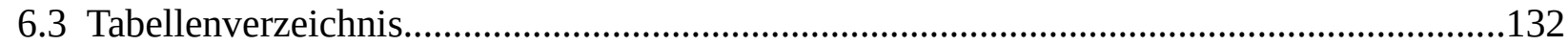

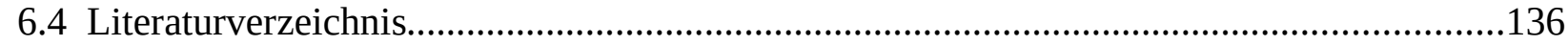

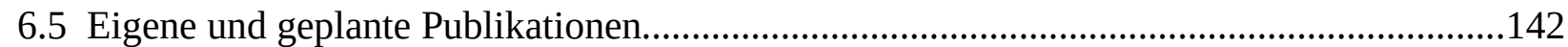

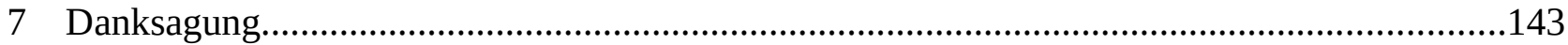

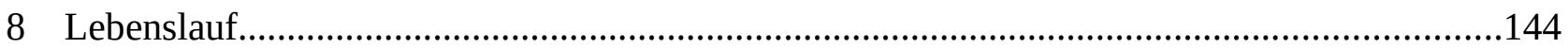

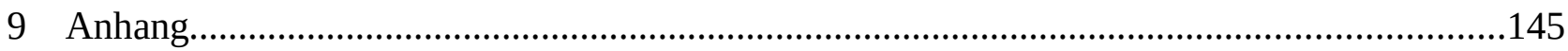

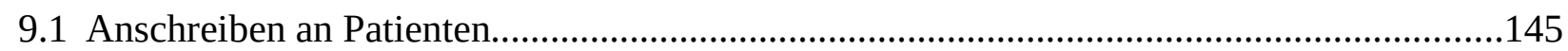

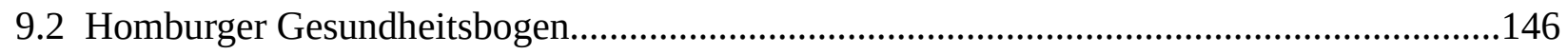

Die im folgenden verwendeten Personalbegriffe beziehen sich gleichermaßen auf Frauen und Männer. 


\section{Zusammenfassung}

\subsection{Summary}

Various risk factors, such as skin type, eye colour, number of melanocytic nevi and, especially, chronic UV exposure increase the possibility of developing skin cancer [80, 26, 27]. It is essentially the responsibility of the individual patient to minimize this risk (i.e. by reducing overall UV exposure). There are also indications that a healthy lifestyle (sufficient exercise, healthy diet, nicotine abstinence, etc.) can help decrease the risk of developing skin cancer [39, 35, 66].

The purpose of our study was to examine to which extent patients who have a history of skin cancers have adopted such preventive measures and as to whether a more intensive program of consultations with patients could lead to improvement in their compliance.

This retrospective study included all patients $(n=653)$ who had been hospitalized in the Dermatology Department, Saarland University Hospital in 2013 (1.1.-31.12.2013) with a skin tumor. In 2014 all these patients were contacted by means of two questionnaires (Homburger Gesundheitsbogen and WHOQOL-BREF). These questionnaires examined 1) whether the patients had improved their health regime (UV exposure, nutrition, regular medical check-ups, physical activity, etc.) after they had been diagnosed with skin cancer and 2) whether the diagnosis of skin cancer had had an influence on the patient's quality of life.

263 patients responded (response rate 40.3\%). 62.0\% $(n=163)$ were male and 38.0\% ( $n=100)$ female. The average age was 69.8 years. $30.0 \%(n=79)$ of patients had a melanocytic tumor, $53.6 \%$ $(n=141)$ a non-melanocytic tumor and $16.3 \%(n=43)$ had both melanocytic and non-melanocytic tumors.

It was encouraging to note that many patients had adopted the recommended action regarding exposure to the sun. After diagnosis $88.5 \%(n=232)$ had reduced their overall exposure to the sun, $90.2 \%$ ( $n=207)$ were less likely to leave the house at midday when the exposure to UV was greatest, $66.8 \%$ ( $n=181$ ) were more likely to use sunscreen, $58.1 \%$ were more likely to protect themselves by wearing a hat and $57.2 \%(n=150)$ wore sunglasses more regularly than they had before diagnosis.

Over half of our patients had changed to a healthier diet and reduced their alcohol consumption after diagnosis (diet changes - 58.2\%, n=154 and reduced alcohol consumption 60.9\%, n=157).

The take up of skin cancer screening and, in particular, follow up examinations was encouraging. 
97.6\% ( $\mathrm{n}=83$ ) of younger patients regularly attended follow up examinations after the diagnosis of skin cancer. Older patients (over 66 years) only attended follow up examinations in $84.8 \%$ of cases ( $n=145, p=0.001$ ). Our results showed that, especially in younger patients, the need for protective measures was well understood whilst older patients with non-melanocytic tumors would benefit from closer patient - physician contact and a greater frequency of medical check-ups. There was no correlation between education, gender or marital status with regard to compliance in attending follow-up examinations.

Surprisingly, the satisfaction of our patients with their quality of life was greater than that of the German population in general. This heightened satisfaction was particularly pronounced in terms of our patients' environment $(\mathrm{p}<0.001)$, their psychological status $(\mathrm{p}=0.001)$ and their social relationships $(\mathrm{p}<0.001)$. Being female, living with a partner, a high level of education and older age had a positive effect on the quality of life of our patients.

Nevertheless, there are still areas (i.e. wearing sun protective clothing) and other risk factors, which patients might not immediately associate with skin cancer. For example, only $25.2 \%$ ( $\mathrm{n}=66$ ) of our patients had increased their level of physical activity after diagnosis. Clearly, therefore, this indicates the need for more education about the causes of skin cancer, as well as warnings about the grave consequences of inattention to the risk factors.

The impact of skin cancer on quality of life was lower than we expected. Although this is positive, it could be the reason for the reduced motivation to make lifestyle changes. The reluctance to adopt health promoting lifestyle changes poses an interesting challenge.

Further studies would be useful to confirm our findings and to evaluate the educational activities already being undertaken in the Saarland. It would also be valuable to identify meaningful measures that could help improve patient compliance in the areas we have identified. 


\subsection{Zusammenfassung}

Verschiedene Risikofaktoren, wie Hauttyp, Augenfarbe, Anzahl vorbestehender melanozytärer Nävi und insbesondere eine chronische UV-Exposition begünstigen die Entstehung von Hautkrebs [80, 26, 27]. Hier liegt es vor allem bei den Patienten, beispielsweise durch Reduzierung der UVExposition, ihr Risiko, an Hautkrebs zu erkranken, zu minimieren. Außerdem gibt es Hinweise darauf, dass sich ein gesunder Lebensstil (ausreichend Bewegung, gesunde Ernährung, Nikotinkarenz, etc.) präventiv auf die Entstehung von Hautkrebs auswirken kann [39, 35, 66].

Ziel unserer Studie war es, zu prüfen, inwiefern Patienten - mit Hauttumoren in der Eigenanamnese - solche präventiven Maßnahmen bereits umsetzten und ob es Ansatzpunkte gibt, bei denen eine intensivere Beratung der Patienten zur Verbesserung ihrer Compliance führen könnte.

In dieser retrospektiven Studie wurden alle Patienten ( $n=653)$ erfasst, die im Jahr 2013 (1.1.31.12.2013) auf Grund einer Hauttumorerkrankung in der Hautklinik des Universitätsklinikums des Saarlandes stationär behandelt wurden. 2014 wurden diese Patienten mittels zweier Fragebögen (Homburger Gesundheitsbogen und WHOQOL-BREF) angeschrieben. Diese Fragebögen prüften zum einen, ob die Patienten, nach Diagnosestellung, ihr Gesundheitsverhalten (UV-Exposition, Ernährung, Vorsorgeuntersuchungen, körperliche Bewegung, etc.) nach Tumordiagnose/-Operation geändert hatten und zum anderen, ob die Diagnose Hautkrebs Einfluss auf die Lebensqualität der Patienten hatte.

Es antworteten 263 Patienten (Rücklaufquote 40,3\%). 62,0\% (n=163) der Patienten waren männlich, 38,0\% ( $n=100)$ weiblich. Der Altersdurchschnitt lag bei 69,8 Jahren. 30,0\% (n=79) der Patienten litten unter einem melanozytären Tumor, 53,6\% (n=141) unter einem nicht melanozytären Turmor und 16,3\% $(n=43)$ der Patienten hatten sowohl einen melanozytären als auch einen nicht melanozytären Tumor.

Sehr erfreulich war die Erkenntnis, dass in Bezug auf Sonnenexposition viele unserer Patienten das empfohlene Verhalten umsetzten. So reduzierten 88,5\% $(n=232)$ ihre Sonnenexposition und 90,2\% ( $n=207)$ achteten darauf, das Haus zur Mittagszeit seltener zu verlassen als vor der Diagnosestellung. 66,8\% ( $\mathrm{n=181)}$ trugen nach Bekanntwerden der Hauttumorerkrankung häufiger Sonnencreme auf, 58,1\% schützen sich gegen eine erhöhte UV-Exposition mit Sonnenhüten und $57,2 \%(n=150)$ mit Sonnenbrillen. 
Auch der Notwendigkeit einer bewussteren Ernährung und Reduzierung des Alkoholkonsums begegnete über die Hälfte (Ernährung - 58,2\%; n=154 und Alkoholkonsum 60,9\%; n=157) unserer Patienten mit Einsicht und verwirklichte eine Besserung des Lebensstils nach Diagnosestellung.

Die Hautkrebsvorsorge und insbesondere die Nachsorge wurden in erfreulich hohem Maße wahrgenommen. 97,6\% (n=83) der jüngeren Patienten gingen nach der Diagnose der Hauttumorerkrankung regelmäßig zur Nachsorge. Bei den älteren Patienten waren es 84,8\% $(n=145 ; \mathrm{p}=0,001)$. Somit traf vor allem bei jungen Patienten die Notwendigkeit dieser Schutzmaßnahmen auf gute Resonanz. Insbesondere ältere Patienten mit nicht-melanozytärem Tumor würden von einer engeren medizinischen Anbindung und einer größeren Intensität ärztlicher Kontrollen profitieren. Eine Abhängigkeit vom Bildungsstand, Geschlecht oder dem Familienstand ergab sich nicht.

Erstaunlicherweise war die Zufriedenheit unserer Patienten mit ihrer Lebensqualität größer als die der deutschen Allgemeinbevölkerung. Diese größere Zufriedenheit war in Bezug auf die Umwelt $(\mathrm{p}<0,001)$, die psychische Verfassung $(\mathrm{p}=0,001)$ und ihre sozialen Beziehungen $(\mathrm{p}<0,001)$ besonders ausgeprägt. Weibliches Geschlecht, das Leben mit einem Partner, ein hohes Bildungsniveau sowie höheres Lebensalter wirkten sich bei unseren Patienten positiv auf die Lebensqualität aus.

Gleichwohl existieren nach wie vor Bereiche (z.B. textiler Sonnenschutz), sowie andere, von den Patienten nicht direkt in Verbindung mit Hautkrebs gebrachte Risikofaktoren (beispielsweise bewegten sich nur 25,2\% ( $\mathrm{n=66)}$ unserer Patienten nach Diagnosestellung mehr als davor), die eindeutig großen Handlungsbedarf für eine intensivere Aufklärung der Patienten erfordern.

Die Auswirkungen der Hautkrebserkrankung auf die Lebensqualität waren geringer als von uns erwartet. In diesem an und für sich erfreulichen Zustand sehen wir einen möglichen Ursprung für den verminderten Antrieb zur Lebensstilveränderung. Die daraus erwachsende Zurückhaltung gegenüber gesundheitsfördernden Veränderungen stellt eine interessante Herausforderung dar.

Weitere Studien wären sinnvoll, um unsere Ergebnisse ggf. zu bestätigen, aber auch um Kenntnis über die Tiefe bereits stattfindender Aufklärungsarbeit im Saarland zu erlangen. Des Weiteren wäre die Feststellung von sinnvollen Maßnahmen, welche geeignet sind, die Compliance der Patienten in den von uns identifizierten Bereichen zu verbessern, von Wert. 


\section{Einleitung}

\subsection{Ziele und Fragestellungen dieser Arbeit}

Diese Arbeit soll eruieren, inwieweit Patienten nach Bekanntwerden und Behandlung ihrer Hautkrebserkrankung ihr Gesundheitsverhalten anpassen.

In jüngerer Zeit konnte für die meisten epithelialen und nicht-epithelialen Hauttumore ein kausaler Zusammenhang mit chronischer UV-Exposition nachgewiesen werden [80]. Kraft Logik sollte sich somit zunehmend, insbesondere vor dem Hintergrund einer intensiven Öffentlichkeitsarbeit und Aufklärung i.S.e. Prävention, eine gesteigerte UV-Prävention in der Bevölkerung - und vor allem bei Patienten mit Hauttumoren in der Eigenanamnese - durchsetzen. Im Fokus dieser Studie stand daher die Frage, ob die Vermeidung der UV-Exposition mittels Sonnencremes, sowie textiler Schutzmittel erfolgte, und ob eine Vermeidung der maximalen Sonneneinstrahlung, u.a. durch ein verändertes Freizeitverhalten, stattfand. Gleichermaßen sollte geprüft werden, ob in den Bereichen „körperliche Bewegung“ und „Ernährung“ eine intensivere Beratung der Patienten im Sinne einer gesünderen Lebensweise notwendig und sinnvoll sein würde.

Darüber hinaus war die Lebensqualität der an Hauttumoren erkrankten Patienten im Vergleich zu der der deutschen Allgemeinbevölkerung von Interesse. Hierbei sollte in 5 verschiedenen Domänen geprüft werden, ob und inwieweit die Hautkrebserkrankung Einfluss auf die Lebensqualität hatte.

Es stand die Frage im Vordergrund, ob es Hinweise gab, dass sich die Diagnose Hautkrebs auf die Lebensqualität auswirkt und, darauf fußend, ob es neue Ansätze gibt, die Compliance und die ArztPatientenbeziehung zu stärken?

\subsection{Einteilung und Epidemiologie von Hauttumoren}

\subsubsection{Einteilung von Hauttumoren}

Generell muss zwischen zwei Arten von Hautkrebserkrankungen differenziert werden. Dem Melanom (nicht epithelialen Hautkrebs) einerseits und den nicht-melanozytären Tumoren (epithelialen Hautkrebsformen) andererseits. Letztere werden in Basalzellkarzinome (Basaliome, 80\%), Plattenepithelkarzinome (20\%), sowie in seltene epitheliale Hautkrebsformen (1\%) unterteilt [43].

Diese Einteilung gilt auch für die vorliegende Studie, in der die Patienten unter anderem nach der Art des Hauttumors (melanozytär oder nicht-melanozytär) aufgeteilt und verglichen wurden. 


\subsubsection{Epidemiologie in Deutschland}

\subsubsection{Krebserkrankungen im Allgemeinen}

Laut statistischem Bundesamt sind bösartige Neubildungen in Deutschland direkt nach kardiovaskulären Erkrankungen die häufigste Todesursache. Im Jahr 2013 waren 39,7\% der Todesfälle auf Herz-Kreislauf Erkrankungen und 25,0\% auf bösartige Neubildungen zurückzuführen [72]. In einer Pressemitteilung des statistischen Bundesamts aus dem Jahr 2013 wird - bei den zum Tode führenden Krebserkrankungen - ein Anstieg von 25\% in den vergangenen 30 Jahren genannt. Bei den Herz-Kreislauferkrankungen mit Todesfolge ist im Vergleich dazu ein Rückgang von ca. 23 \% zu verzeichnen [71]. Auch in der aktuellen Statistik von 2015 zeigen sich ähnliche Zahlen [74]. Eine graphische Darstellung findet sich in Abbildung 2.1.

Todesursachen nach Krankheitsarten 2015

$\%$

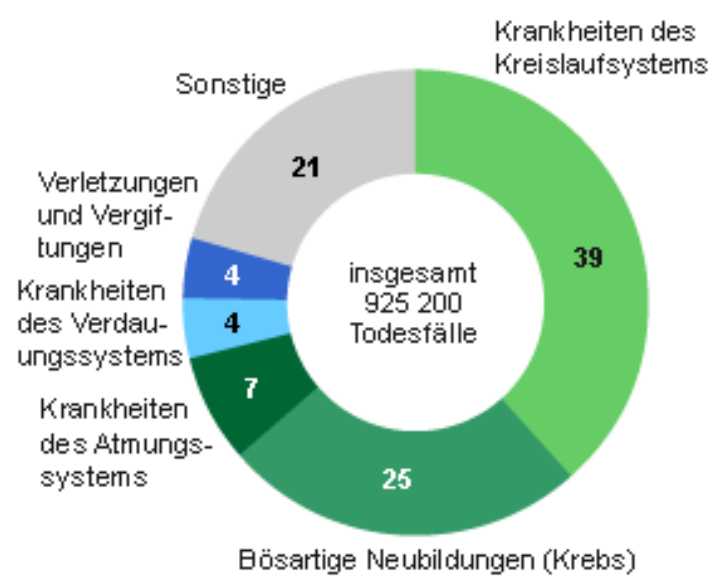

(6) It Statistisches Bundesarnt (Destatis), 2017
Abbildung 2.1: Todesursachen nach Krankheitsarten 2015 (Statistisches Bundesamt (Destatis), 2017. In www.destatis.de (Thematische Recherche: Zahlen \& Fakten Gesellschaft \& Staat - Gesundheit Todesursachen - Dokumentart: Abbildung). Abrufdatum: 09.10.2017

\subsubsection{Hautkrebs im Speziellen}

Nachfolgend wird nun die Häufigkeit von Hautkrebserkrankungen mit dem Ziel diskutiert, die Bedeutung dieser Erkrankung für die Gesellschaft und insbesondere für das Gesundheitssystem aufzuzeigen.

Bei der Betrachtung der Inzidenzrate von Hauttumoren fällt auf, dass diese in den vergangenen Jahrzehnten stetig angestiegen ist [29]. Allerdings kann eine genaue Erfassung der epidemiologischen Daten zu Hautkrebserkrankungen z.Zt. noch nicht vorgenommen werden, da die Registrierung von Hautkrebserkrankungen in Tumorregistern in einigen Bundesländern (z.B. 
Saarland, Hamburg) bereits seit längerem, in anderen Bundesländern (z.B. Baden-Württemberg) erst seit kurzem stattfindet. Daher gibt es bezüglich der Häufigkeit der unterschiedlichen Hautkrebsentitäten in Deutschland bislang keine flächendeckenden Daten [47, 20].

Im Jahre 2012 wurden in einer Studie sowohl die Daten aus dem Krebsregister Schleswig-Holsteins (KRSH), als auch die Schätzungen des Zentrums für Krebsregisterdaten, die Daten der Gesellschaft des epidemiologischen Krebsregisters (GEKID) und schließlich die Todesursachenstatisktik des statistischen Bundesamtes zusammengeführt. Daraus geht hervor, dass in Deutschland jährlich etwa 18000 Patienten an einem invasiven malignen Melanom erkrankten. Hinzu kamen etwa 8400 Patienten jährlich mit einem in-situ Melanom. Bei den epithelialen Hautkrebsentitäten geht man jährlich von über 150000 Neuerkrankungen aus. Der größte Teil davon waren Basalzellkarzinome mit jährlich etwa 130000 Neuerkrankungen. Den kleineren Anteil repräsentierten Plattenepithelkarzinome mit etwa 10000-30000 Neuerkrankungen p.a. [47].

Das mittlere Erkrankungsalter für das maligne Melanom lag laut Robert-Koch-Institut 2014 für Männer bei 67 vis-à-vis bei 60 Jahren für Frauen. Für das Basaliom lag das mittlere Erkrankungsalter für Männer bei 72 und für Frauen bei 71 Jahren, beim Plattenepithelkarzinom für Männer bei 77 vis-à-vis bei 79 Jahren für Frauen [63].

Nach Einführung des bundesweiten Hautkrebsscreeningprogramms im Jahr 2008 zeigte sich ein weiterer Anstieg der Inzidenz, da nun mehr Patienten zuverlässig untersucht und erfasst werden konnten. So gibt es seither in Deutschland ein Programm, bei dem gesetzlich versicherte Patienten ab dem 35. Lebensjahr im zweijährigen Turnus durch ihren Hausarzt oder einen Dermatologen am ganzen Körper auf tumorsuspekte Hautauffälligkeiten hin untersucht werden [46, 22].

In einem Ranking aller Krebsarten (ausgenommen nicht-melanozytärer Hautkrebs) lag die Inzidenzrate für das maligne Melanom im Jahr 2014 sowohl bei Männern als auch Frauen an fünfter Stelle [63]. Somit sind in Deutschland etwa 4\% aller Krebsneuerkrankungen Melanome [47]. Etwa 1\% der durch Krebs verursachten Todesfälle sind auf Hautkrebs, insbesondere auf maligne Melanome, zurückzuführen [46].

Bei den nicht-melanozytären Tumoren ist die Inzidenz noch um ein vielfaches höher. So liegt die Neuerkrankungsrate pro Jahr bei Männern in etwa auf Höhe der Inzidenz des Prostatakarzinoms (häufigster Tumor des Mannes). Bei Frauen schätzt man, dass die Inzidenz des nicht-melanozytären Hautkrebses zwischen der von Brustkrebs (1. Stelle) und Darmkrebs (2.Stelle) einzuordnen ist [20]. 
Kosten und Aufwand für das Gesundheitssystem sind enorm. 2003 wurden über 20.000 Patienten mit Melanomen stationär behandelt. Bei nicht-melanozytären Hauttumoren waren es mehr als 41.000 stationär behandelte Patienten [68].

\subsection{Wichtige Risikofaktoren für die Entwicklung eines Hauttumors}

Für die verschiedenen Hauttumore existieren zahlreiche Risikofaktoren. Davon können die meisten durch Veränderungen des Lebensstils vermieden oder zumindest beeinflusst werden, da sie umweltbedingt sind. Ein wichtiger Aspekt unserer Studie war daher die Frage, ob die Patienten nach Diagnosestellung eines Hauttumors ihr persönliches Risikoprofil minimieren würden. In diesem Zusammenhang werden die wichtigsten Risikofaktoren erläutert und ihre Bedeutung für die Entstehung von Hautkrebs herausgearbeitet.

\subsubsection{UV-Strahlung}

Die Exposition gegenüber Sonnenlicht und der damit verbundenen UV-Strahlung stellt für jede Form des Hautkrebses den bedeutendsten Risikofaktor dar [80, 3].

Es konnte gezeigt werden, dass durch die Exposition mit UV-Strahlung das Risiko für die verschiedenen Hautkrebsarten je nach Lebensalter und Dauer der Exposition („kumulativ“ oder „intermittierend“) unterschiedlich steigt. Während die dauerhafte Einwirkung von UV-Strahlung über das gesamte Leben („,kumulative Exposition“) einen Risikofaktor für das Plattenepithelkarzinom darstellt, spielen beim Basalzellkarzinom und Melanom vor allem Sonnenbrände und die zeitweise Exposition mit UV-Strahlung (,intermittierende Exposition“) eine Rolle [20, 3].

Ebenso ist auch das Lebensalter, in dem die Exposition mit UV-Strahlung stattfindet, wichtig. So wurde in einer 2014 durchgeführten Studie gezeigt, dass kontinuierliche Sonnenexposition über das gesamte Leben mit einer erhöhten Inzidenz von Basalzellkarzinomen und Plattenepithelkarzinomen vergesellschaftet ist, wohingegen das Risiko, an einem Melanom zu erkranken, vor allem durch Sonnenbrände in der Pubertät und Kindheit erhöht wird [84, 80].

Freizeitaktivitäten oder Berufe, die vorwiegend im Freien stattfinden (und somit mehr UVExposition zur Folge haben), erhöhen das Risiko, im Laufe des Lebens einen Hauttumor zu verursachen [80]. So konnte beispielsweise gezeigt werden, dass Gärtner ein erhöhtes Risiko aufweisen, an Basalzellkarzinomen und Plattenepithelkarzinomen zu erkranken [51]. 
Auch bei Solariumbesuchen kommt es zur Exposition der Haut mit UV-Strahlung. Hier konnte in verschiedenen Studien ein Zusammenhang zwischen Hautkrebs und Solariumbesuchen festgestellt werden [80, 34].

\subsubsection{Hauttyp und Augenfarbe}

Menschen mit einem hellen Hauttyp neigen eher dazu, im Laufe ihres Lebens an Hautkrebs zu erkranken. Auch zwischen Haarfarbe und dem Erkrankungsrisiko besteht ein Zusammenhang. Menschen mit roten oder blonden Haaren gelten als gefährdeter als andere. Ähnliches gilt für die Augenfarbe. Menschen mit grünen oder blauen Augen bekommen häufiger Hautkrebserkrankungen als solche mit anderen Augenfarben [80, 26, 27]. In Abbildung 2.2 findet sich eine Übersicht der unterschiedlichen Hauttypen.

\begin{tabular}{|c|c|c|c|c|c|c|}
\hline Hauttyp & I & II & III & IV & $\mathbf{V}$ & VI \\
\hline \multicolumn{7}{|l|}{ Beschreibung } \\
\hline Natürliche Hautfarbe: & sehr hell & hell & $\begin{array}{l}\text { hell bis } \\
\text { hellbraun }\end{array}$ & $\begin{array}{l}\text { hellbraun, } \\
\text { oliv }\end{array}$ & $\begin{array}{l}\text { dunkel- } \\
\text { braun }\end{array}$ & $\begin{array}{l}\text { dunkel- } \\
\text { braun bis } \\
\text { schwarz }\end{array}$ \\
\hline $\begin{array}{l}\text { Sommersprossen/ } \\
\text { Sonnenbrandflecken: }\end{array}$ & sehr häufig & häufig & selten & keine & keine & keine \\
\hline Natürliche Haarfarbe: & $\begin{array}{l}\text { rötlich bis } \\
\text { rötlich- } \\
\text { blond }\end{array}$ & $\begin{array}{l}\text { blond bis } \\
\text { braun }\end{array}$ & $\begin{array}{l}\text { dunkel- } \\
\text { blond bis } \\
\text { braun }\end{array}$ & $\begin{array}{l}\text { dunkel- } \\
\text { braun }\end{array}$ & $\begin{array}{l}\text { dunkel- } \\
\text { braun bis } \\
\text { schwarz }\end{array}$ & schwarz \\
\hline Augenfarbe: & blau, grau & $\begin{array}{l}\text { blau, grün, } \\
\text { grau, } \\
\text { braun }\end{array}$ & $\begin{array}{l}\text { grau, } \\
\text { braun }\end{array}$ & $\begin{array}{l}\text { braun bis } \\
\text { dunkel- } \\
\text { braun }\end{array}$ & $\begin{array}{l}\text { dunkel- } \\
\text { braun }\end{array}$ & $\begin{array}{l}\text { dunkel- } \\
\text { braun }\end{array}$ \\
\hline \multicolumn{7}{|l|}{ Reaktion auf die Sonne } \\
\hline Sonnenbrand: & $\begin{array}{l}\text { immer und } \\
\text { schmerz- } \\
\text { haft }\end{array}$ & $\begin{array}{l}\text { fast immer, } \\
\text { schmerz- } \\
\text { haft }\end{array}$ & $\begin{array}{l}\text { selten bis } \\
\text { mäßig }\end{array}$ & selten & sehr selten & $\begin{array}{l}\text { extrem } \\
\text { selten }\end{array}$ \\
\hline Bräunung: & keine & $\begin{array}{l}\text { kaum bis } \\
\text { mäßig }\end{array}$ & $\begin{array}{l}\text { fort- } \\
\text { schreitend }\end{array}$ & $\begin{array}{l}\text { schnell und } \\
\text { tief }\end{array}$ & keine & keine \\
\hline $\begin{array}{l}\text { Erythemwirksame } \\
\text { Schwellenbestrahlung: }\end{array}$ & $200 \mathrm{Jm}-2$ & $250 \mathrm{Jm}-2$ & $350 \mathrm{Jm}-2$ & $450 \mathrm{Jm}-2$ & $800 \mathrm{Jm}-2$ & $\begin{array}{l}>1000 \\
\mathrm{Jm}-2\end{array}$ \\
\hline
\end{tabular}

Abbildung 2.2: Hauttypen, Quelle:[20] 


\subsubsection{Nävi}

Große kongenitale Nävi stellen einen Risikofaktor für das maligne Melanom dar [48]. Auch die Anzahl der erworbenen Nävi und atypische Pigmentmale stellen einen Risikofaktor für das Melanom dar [20].

\subsection{4 weitere Risikofaktoren}

\subsubsection{Nikotin}

Aus verschiedenen Studien ging hervor, dass Nikotinkonsum ein erhöhtes Risiko für ein Plattenepithelkarzinom mit sich bringt [35]. Es gibt auch Hinweise, dass das Rauchen die Entstehung von malignen Melanomen fördert. Der zugrundeliegende Pathomechanismus ist bislang nicht abschließend geklärt [67].

\subsubsection{Alkohol}

Eine Korrelation zwischen Alkoholkonsum und einem erhöhten Risiko für schwere Sonnenbrände konnte bereits nachgewiesen werden. Es wurde diskutiert, ob Personen, die Alkohol getrunken hatten, die direkte Sonnenexposition als weniger drastisch wahrnahmen und somit keine Verhaltensänderungen zur Prävention aufwiesen. Eine längere Sonnenexposition als bei Personen, die keinen Alkohol getrunken hatten, konnte allerdings nicht beobachtet werden [82]. Trotzdem scheint es wahrscheinlich, dass Alkohol ein Risikofaktor für Hautkrebserkrankungen ist. So fand sich unter anderem bei Patienten, die regelmäßig Wein getrunken hatten, ein gehäuftes Auftreten von Basalzellkarzinomen [41]. WU et al. fand ähnliche Ergebnisse. Ob es sich um die oben genannte Korrelation zwischen Alkoholkonsum und schweren Sonnenbränden oder ggf. auch um Effekte durch Abbauprodukte des Alkohols handelt, die die Entstehung von Hautkrebs begünstigen, wird noch diskutiert [85].

\subsubsection{Ernährung}

Unklar ist noch, ob sich die Art der Ernährung auf die Entstehung von Hautkrebs auswirkt.

IBIEBELE et al. zeigten, das eine fett- und fleischreiche Ernährung die Entstehung von Plattenepithelkarzinomen begünstigen kann [39]. GAMBA et al. konnten diesen Zusammenhang nicht bestätigen [28]. TANG et al. zeigten sogar, dass ein erhöhter BMI mit einem erniedrigten Risiko für nicht melanozytäre Tumoren einherging, was allerdings in Zusammenhang damit gebracht wurde, dass die übergewichtigen Patienten weniger Aktivitäten im Freien nachgingen und das Haus daher seltener verließen. Insofern hatten sie eine geringere UV-Exposition, wodurch ihr Risiko gegenüber den anderen Patienten gesenkt wurde [75]. 


\subsection{Prävention von Hautkrebserkrankungen - mögliche Ansatzpunkte}

Es gibt viele Möglichkeiten der Primärprävention von Hautkrebs. Vorrangig ist der Schutz vor UVStrahlung.

Das Bundesamt für Strahlenschutz (BFS) empfiehlt folgende Verhaltensregeln [12]:

- Sonne in den Mittagsstunden vermeiden

- Schutz durch Textilien (bspw. langarmiges Hemd)

- Schutz der Augen

- $\quad$ keine Sonnenbrände riskieren

- das Aufbringen von Sonnencremes

- beim Schwimmen ausreichend Badebekleidung tragen

- Solarien meiden

- darauf achten, dass Kinder ebenfalls ausreichend vor der Sonne geschützt sind

- Vorbild sein

In den Leitlinien zur Prävention von Hautkrebs der Deutschen Krebsgesellschaft wird erwähnt, dass der textile Schutz und das Vermeiden voller Sonneneinstrahlung (z.B. in der Mittagssonne, Freizeitaktivitäten in der Sonne) Vorrang vor dem topischen Schutz durch Sonnencremes hat. Personen, die auf Grund ihres Berufes erhöhter UV-Exposition ausgesetzt sind, sollten die Belastung so gering wie möglich halten. Dies könne beispielsweise durch Pausen und eine gute Strukturierung der Arbeit geschehen, damit die maximale Sonneneinstrahlung am Mittag gemieden werden kann. Gleiches gilt für Kindertagesstätten und Schulen. Kinder sollten bereits im Schulalter über die verschiedenen Präventionsmaßnahmen aufgeklärt werden. Auch bei erwachsenen Patienten sollte der behandelnde Arzt bei den regelmäßigen Screening Untersuchungen auf die empfohlenen Verhaltensregeln hinweisen [20].

\section{Protektive Wirkung von Sonnenschutzcremes:}

In einer Studie von GREEN et al. konnte gezeigt werden, dass die tägliche Anwendung von Sonnenschutzcremes eine protektive Wirkung hatte und die Entstehung von Plattenepithelkarzinomen im besten Fall verhindern konnte. Im Rahmen der Studie waren die 
Patienten über einen Zeitraum von 4,5 Jahren beobachtet worden [32]. Es wurde kein Effekt auf die Prävention von Basalzellkarzinomen beobachtet, wobei eine präventive Wirkung als wahrscheinlich erachtet wird [16]. Im Verlauf konnte in einer Follow-Up Studie gezeigt werden, dass sich die tägliche Applikation von Sonnencremes auch positiv auf die Vermeidung von Melanomen auswirkte [33]. Personen, die Sonnenschutzcremes aufgetragen hatten, schienen sich jedoch länger in der Sonne aufzuhalten als solche, die keinen topischen Schutz aufgetragen hatten, da sie sich besser und ausreichend geschützt fühlten [5].

\subsection{Lifestyle changes nach Tumorerkrankungen}

Die WHO geht davon aus, dass 30\% - 50\% aller durch Krebserkrankungen hervorgerufenen Todesfälle durch risikobehaftete Lebensweisen - wie beispielsweise Rauchen, Übergewicht, Alkohol, geringe Bewegung etc. - verursacht werden und daher vermeidbar wären [83]. Insbesondere in Hinblick auf diesen Sachverhalt soll in unserer Studie beurteilt werden, ob Hautkrebspatienten nach Diagnosestellung ihr Gesundheitsverhalten verbessern, indem sie versuchen, die auf sie zutreffenden Risikofaktoren für die Entstehung eines Hautkrebses zu minimieren. Allerdings gibt es nur wenige Studien, aus denen hervorgeht, dass eine Bereitschaft zu einer Verbesserung der Risikofaktoren bei den Patienten überhaupt besteht.

So untersuchte MASER et al. 260 Patienten, die mittels "Mohs Micrographic surgery" (eine spezielle Operationstechnik für Hautkrebs aus dem angloamerikanischen Raum) [59] an Hauttumoren operiert wurden. Viele Patienten, die vor der Operation keine Sonnencreme aufgetragen hatten, benutzten im Anschluss an die Operation Sonnencremes und hielten auch ihre Familienangehörigen zur Benutzung solcher Cremes an. Über 50\% der Befragten untersuchten sich häufiger selbst auf verdächtige Nävi. Auch die Zeit, die bei maximaler Sonneneinstrahlung im Freien verbracht wurde, reduzierte sich [52].

Zum Vergleich: In einer Studie aus dem Jahr 2000, die Brustkrebspatientinnen und Prostatakarzinom-Patienten einschloss, zeigte sich, dass sich die Patienten analog zur Gesamtpopulation zwar deutlich gesünder, jedoch trotzdem immer noch zu ungesund ernährten (weniger als 5 Portionen Früchte und Gemüse pro Tag). Es gab weiterhin Bereiche (z.B. Bewegungsverhalten), in denen Änderungen hätten stattfinden sollen [19]. Auch MASER et al. (s.o.) erkannten kein signifikant besseres Verhalten der Patienten in den Bereichen Rauchen, Ernährung oder Alkoholkonsum.

Ähnliches zeigte sich bei BLANCHARD et al., die Patienten mit Brustkrebs, Prostatakrebs, 
colorektalen Karzinomen, Blasentumoren, Uteruskarzinomen und Melanomen untersuchten. Von den 9105 untersuchten Patienten ernährten sich nur knapp 20\% gesünder als vor der Tumorerkrankung. Ähnlich verhielt es sich bei körperlichen Aktivitäten. Insgesamt nur 5\% der Patienten führten gesundheitsfördernde Veränderungen in allen 3 Bereichen ihres Lebensstils (Rauchen, Ernährung, Bewegung) durch [10].

In einer 2003 durchgeführten Studie (50\% Brustkrebspatienten, 50\% andere Krebsarten) zeigte sich, dass nur 46\% der Raucher nach der Diagnose mit dem Rauchen aufgehört hatten. Nur 15,7\% der Patienten bewegten sich mehr als vorher - und erstaunlicherweise bewegten sich $30,1 \%$ nach der Diagnosestellung sogar weniger [11].

Teilweise verhielten sich Tumorpatienten sogar ungesünder als Gesunde. So untersuchten RAUSCH et al. Patientinnen, die an einem Mamma-Karzinom Screening teilnahmen. Es wurden alle Patientinnen befragt, unabhängig von der Krebsart, oder ob sie erkrankt oder gesund waren. Patientinnen, die in Ihrer Vorgeschichte eine Krebserkrankung hatten, wiesen schlechtere Verhaltensweisen auf als solche ohne frühere Krebserkrankung. Unter den Patientinnen mit Krebserkrankung rauchten mehr und achteten weniger auf ihre Gesundheit als Patientinnen in der Vergleichsgruppe. Auch war es wahrscheinlicher, dass sie an Gewicht zugenommen hatten [61].

Aus den oben genannten Studien geht hervor, dass einige Patienten nach Diagnosestellung bereit sind, ihre Risikofaktoren zu minimieren. Umso mehr erstaunt es, dass es viele Patienten gibt, die ihr gesundheitsschädliches Verhalten fortsetzen. Es stellt sich daher die Frage, ob eine Änderung/Verbesserung des Lebensstils durch Motivation und bessere Beratung der Patienten gefördert werden kann.

Indes kann festgestellt werden, dass zahlreiche Patienten selbst eine große Bereitschaft zur Verbesserung ihres Lebensstils erkennen ließen. DEMARK-WAHNEFRIED et al. zeigten, dass bis zu 80\% der untersuchten Krebspatienten daran interessiert waren, an Gesundheitsprogrammen teilzunehmen [19].

Die Beratung durch den Arzt findet allerdings häufig in zu geringem Ausmaß statt. In der Studie von BLANCHARD et al. hatten die behandelnden Ärzte nur bei 22\% der Patienten eine Ernährungsumstellung empfohlen; nur 19\% der Patienten hatten die Empfehlung bekommen, sich mehr zu bewegen. Dies scheint umso bedauerlicher, da die Empfehlung des Arztes, ungeachtet der Tatsache, dass sie nur selten ausgesprochen wurde, einen Einfluss auf das Bewegungs- und 
Ernährungsverhalten der Patienten hatte [11].

Die enorme Bedeutung einer Beratung zeigt sich darin, dass Patienten, die ihr Verhalten positiv verändert hatten, sich als Folge einer höheren Lebensqualität erfreuten als solche, die ihr Verhalten beibehalten hatten. Je mehr sich Patienten in einem gesundheitsfördernden Sinn veränderten, desto positiver nahmen sie ihre Lebensqualität wahr [10]. Körperliche Bewegung wirkte sich sogar positiv auf Behandlung und Nachsorge von Krebserkrankungen aus [78].

\subsection{Lebensqualität}

1946 wurde Gesundheit durch die WHO folgendermaßen definiert: „Gesundheit ist der Zustand des völligen körperlichen, psychischen und sozialen Wohlbefindens und nicht nur das Freisein von Krankheit und Gebrechen“(WHO 1946).

Aus dieser Bestimmung geht hervor, dass auch das psychische und soziale Befinden des Einzelnen eine wichtige Rolle für die Gesundheit spielt. Mit dieser Beschreibung der Gesundheit als Vorlage entstand in den achtziger Jahren der Begriff der Lebensqualität. Es wurde erforderlich, auch das subjektive Empfinden des einzelnen Patienten in die komplette medizinische Behandlung zu integrieren [77].

Das WHOQOL Projekt der WHO hatte die Aufgabe, einen Fragebogen zu entwerfen, mit dem man Lebensqualität messen und somit vergleichbar machen konnte. Dies führte zu einer weiteren Definition: „Lebensqualität ist die individuelle Wahrnehmung der eigenen Lebenssituation im Kontext der jeweiligen Kultur und des jeweiligen Wertesystems und in Bezug auf die eigenen Ziele, Erwartungen, Beurteilungsmaßstäbe und Interessen“ (WHOQOL-Group). In dieser Definition treten schließlich auch der kulturelle Hintergrund sowie die persönlichen Umstände, in denen ein Mensch lebt, in den Vordergrund [2]. Infolgedessen wurde die Erfassung der Lebensqualität ein unentbehrliches Instrument, den Verlauf einer Krankheit sowie den Erfolg einer Therapie genauer einzuordnen [4].

Ein Behandlungserfolg wurde nun also nicht mehr nur am medizinischen Erfolg (Überlebensstatistik, Nebenwirkungen etc.), sondern auch daran, ob der Patient im Anschluss an die Behandlung insgesamt zufrieden war, gemessen. Hinsichtlich der Therapie zeigt sich unter Berücksichtigung der Lebensqualität des Patienten eine deutliche Besserung der Compliance, der Arzt-Patienten-Beziehung und der Zufriedenheit des Patienten mit der Behandlung. Am Beispiel eines onkologischen Palliativpatienten lässt sich dieser Sachverhalt besonders gut darstellen. Indem 
das subjektive Empfinden des Erkrankten in die Behandlung einbezogen wird, lässt sich die Entscheidung über mögliche medizinische und supportive Behandlungsmaßnahmen deutlich erleichtern und verbessern. Eine Lebensverlängerung um wenige Wochen im Rahmen einer Chemotherapie steht unter Umständen in keinem Verhältnis zu den möglichen Nebenwirkungen und dem damit verbundenden Verlust von Lebensqualität [2]. 


\section{Material und Methoden}

\subsection{Gewinnung der Daten}

In dieser retrospektiven Studie wurden alle Patienten erfasst, die im Jahr 2013 (1.1.-31.12.2013) auf Grund einer Hauttumorerkrankung in der dermatologischen Abteilung des Universitätsklinikums des Saarlandes stationär behandelt worden waren. Es wurden die verschiedensten Hauttumorerkrankungen (Melanom, Basaliom, Sarkome, Merkelzellkarzinome etc.) berücksichtigt. 2014 wurden diese Patienten dann mittels zweier Fragebögen (Homburger Gesundheitsbogen und WHOQOL-BREF) angeschrieben und gebeten, diese innerhalb einer Frist von 2 Monaten (Einsendeschluss 31.08.2014) zurückzusenden. Anschreiben und "Homburger Gesundheitsbogen" finden sich im Anhang dieser Dissertation.

Das Anschreiben erfolgte postalisch. Eine Antwort war postalisch oder per Fax möglich. Die Versendung der Bögen erfolgte einmalig, Erinnerungsmaßnahmen erfolgten nicht. Der Versandaktion war die Erhebung folgender Daten aus der Patientenakte vorausgegangen.

- Alter des Patienten

- Geschlecht

- Art des Tumors (melanozytär, nicht-melanozytär oder sowohl als auch)

Fragebögen wurden mit einer Nummer kodiert, so dass mittels einer Entschlüsselung jeder Fragebogen einem Patienten zugeordnet werden konnte. Es war damit gewährleistet, dass die im Vorfeld erhobenen Patientendaten den korrekten Fragebögen zugeteilt werden konnten.

Zwei Kernfragen wurden gestellt:

- Welchen Einfluss hatte die dermatologische Tumorerkrankung auf die Lebensqualität der Patienten?

- Führte die Diagnose der Tumorerkrankung zu einer Änderung im Gesundheitsbewusstsein oder Vorsorgeverhalten der Patienten. Führen die Patienten nach erlittener Hauttumorerkrankung ein gesünderes Leben oder werden „schlechte“ Gewohnheiten beibehalten?

Die Studie wurde durch die Ethik-Kommission der Ärztekammer des Saarlandes geprüft und genehmigt (Ethikvotum, Kenn-Nr. 140/14). 


\subsection{Verwendete Fragebögen}

\subsubsection{Der WHOQOL-BREF}

Der WHOQOL-BREF ist ein Fragebogen zur Erfassung der subjektiven Lebensqualität. Das 1991 von der World Health Organisation initiierte Projekt hatte zum Ziel, Lebensqualität messbar und vergleichbar zu machen. Lebensqualität wurde als individuelle Wahrnehmung der eigenen Lebenssituation im Kontext der jeweiligen Kultur und des jeweiligen Wertesystems sowie in Bezug auf persönliche Ziele, Erwartungen, Beurteilungsmaßstäbe und Interessen gesehen.

Initial erfolgte durch die Arbeitsgruppe die Entwicklung eines ausführlichen Fragebogens (WHOQOL-100), der alle Aspekte der Lebensqualität erfassen sollte. Die Bearbeitungszeit dieses Bogens lag allerdings bei 30-45 Minuten, so dass aus praktischen Gründen eine Kurzversion (WHOQOL-BREF) entwickelt wurde. Durch die kürzere Bearbeitungszeit von nur 5-10 Minuten bot sich dieser Fragebogen an, die Lebensqualität von Teilnehmern beliebiger Studien zu bestimmen und mit der der Allgemeinbevölkerung zu vergleichen. Es wurden insgesamt 26 Fragen konzipiert, die mittels einer fünfstufigen Likert-Skala zu beantworten sind.

Es sind diese 26 Fragen, welche in der Auswertung den folgenden vier Domänen sowie einem Globalwert („allgemeiner Gesundheitszustand“ bzw. „Zufriedenheit mit dem Gesundheitszustand“) zugeordnet werden.

- $\quad$ „Physisch“ (Fragen 3, 10, 16, 15, 17, 4, 18)

- „Psychisch“ (Fragen 5, 7, 19, 11, 26, 6)

- Soziale Beziehungen“ (Fragen 20, 22, 21)

- „Umwelt“ (Fragen 8, 23, 12, 24, 13, 14, 9, 25)

- „Globalwert“ (Fragen 1, 2)

Anschließend erfolgt die Berechnung der „Domänenwerte“, indem man das arithmetische Mittel der zur jeweiligen Domäne gehörenden Fragen mit 4 multipliziert und aus Gründen der Vergleichbarkeit in einen Wertebereich zwischen 0 - 100 überführt (Domäne 0 - $100=$ (Domänenwert -4) 100/16). Es wurden nur Domänen berücksichtigt, in denen höchstens eine Frage der Domäne unbeantwortet geblieben war.

Auf diese Weise konnte aus den erhobenen Daten ein Mittelwert zwischen allen Befragten ermittelt werden; auch Vergleiche zwischen verschiedenen Gruppen (z.B. Patienten mit Melanomen, Patienten mit nicht melanozytären Tumoren) konnten angestellt werden. Es gibt alters- und geschlechtsbezogene Normwerte für die deutsche Allgemeinbevölkerung, die aus Daten von 2073 
Personen ermittelt wurden und dem Vergleich mit den eigenen erhobenen Daten dienen [2].

\subsubsection{Der Homburger Gesundheitsbogen}

Beim Homburger Gesundheitsbogen handelt es sich um einen von uns spezifisch für die vorliegende Studie erdachten Fragebogen. Er dient dem Zweck, Änderungen im Gesundheitsverhalten der Patienten nach Bekanntwerden der Tumorerkrankung zu erfragen.

Mittels insgesamt 15 Fragen (11 Fragen nach Likert-Skala zu beantworten; 4 Fragen mit jeweils zwei Antwortmöglichkeiten) wurden folgende Gesundheitsfaktoren abgefragt:

- Körperliche Bewegung

- Sonnenexposition

- Verwendung von textilen und topischen UV-Schutzmöglichkeiten

- Urlaubsverhalten

- Freizeitverhalten

- Ernährung

- Alkoholgenuss

- Beruf mit erhöhter Sonnenexposition

- Wahrnehmung der Hautkrebsvorsorgeuntersuchung bzw. Hautkrebsnachsogeuntersuchung

- Nikotinkonsum

Hierbei wurde vor allem auf die dynamische Komponente Wert gelegt: hatte sich das o.g. Verhalten durch die Diagnose einer Hauttumorerkrankung geändert? Der Fragebogen ist im Anhang abgebildet.

Wurde eine Frage durch einen Patienten unbeantwortet gelassen, so wurde dieser fehlende Wert bei der Auswertung der Frage nicht berücksichtigt. Bei der jeweiligen Auswertung der Fragen findet sich eine Angabe wie viele Patienten die Frage unbeantwortet ließen.

\subsubsection{Statistik}

Für die Auswertung wurde IBM SPSS Statistics (Version 22) für Windows 7 und Microsoft Excel (2010) verwendet.

Die Auswertung erfolgte in Kooperation mit dem Institut für Medizinische Biometrie, Epidemiologie und Medizinische Informatik des Uniklinikums Homburg/Saar (Universität des Saarlandes). 
P-Werte von kleiner als 0,05 wurden als statistisch signifikant erachtet bei einem zweiseitigen Signifikanzniveau von 5\%. Bei dieser explorativen Studie wurden keine Korrekturen für multiples Testen vorgenommen.

Wie bereits erklärt, hatten wir im Vorfeld aus den Patientenakten Alter und Geschlecht der Patienten, sowie die dermatologische Tumorart bestimmt. Zudem erfragte der WHOQOL-BREF Familienstand und Schulabschluss. Dies ermöglichte einen Vergleich der unterschiedlichen Gruppen miteinander, was wie folgt vonstatten ging:

Alter: Zur besseren Vergleichbarkeit wurden die Patienten beim WHOQOL-BREF, in Gruppen (bspw. zwischen 26-35 Jahren) gefasst. Beim Homburger Gesundheitsbogen erfolgte die Aufteilung in 2 Gruppen. Eine Gruppe schloss Patienten bis zum einschließlich 65. Lebensjahr ein, die andere Gruppe schloss Patienten ab dem 66. Lebensjahr ein.

Geschlecht: Die Trennung erfolgte nach männlich und weiblich.

Schulabschluss: Zur besseren Vergleichbarkeit teilten wir die Patienten in zwei Gruppen ein. Die erste Gruppe bestand aus Patienten, welche als höchste Qualifikation die mittlere Reife absolviert hatten, also entweder keinen Abschluss, einen Hauptschulabschluss oder die mittlere Reife besaßen. Die zweite Gruppe bestand aus Patienten, die einen höheren Abschluss als die mittlere Reife hatten, also mit Fachhochschulreife, Abitur, Fachhochschulabschluss, universitärer oder postgraduierter Qualifikation. Unter postgraduierter Qualifikation wurde eine Promotion oder Professur verstanden.

Familienstand: Auch hier erfolgte die Aufteilung der Patienten in zwei Gruppen. In die erste Gruppe kamen Patienten ohne Partner (verwitwet, getrennt lebend, geschieden und allein lebend); in einer zweiten Gruppe befanden sich Patienten, die mit einem Partner zusammen lebten (verheiratet und mit Partner lebend).

Tumorentität: Diesbezüglich wurden Patienten entsprechend der Histologie des Tumors der Gruppe melanozytärer Tumor oder nicht-melanozytärer Tumor zugeordnet. Litten Patienten unter melanozytärem und nicht-melanozytärem Tumor, wurden sie einer weiteren Gruppe „beide Tumorentitäten“ zugeordnet.

\subsubsection{Statistische Auswertung des WHOQOL-BREFS}

Zum Vergleich der Mittelwerte des WHOQOL-Bref mit den in dieser Studie erhobenen Werten diente der Einstichproben t-Test. Allerdings führten wir zur besseren Vergleichbarkeit noch eine 
Altersstandardisierung durch. Lag in unserer Studie der Altersdurchschnitt bei 69,8 Jahren, war dieser bei der Normstichprobe des WHOQOL-BREF deutlich jünger. Die Altersstandardisierung erfolgte auf dem Wege einer Berechnung des Prozentanteils unserer Patienten an einer gewissen Altersgruppe (bspw. 26-35 Jahre). Die Mittelwerte des WHOQOL-BREF wurden je nach Altersgewichtung unseres Fragebogens ebenso verteilt. Auf diese Weise ergaben sich die auf unser Studienkollektiv altersstandardisierten WHOQOL-BREF Normwerte. Damit waren die Mittelwerte der nun auf unsere Studie altersstandardisierten Normstichprobe des WHOQOL-BREF mit den von uns erhobenen Mittelwerten vergleichbar.

In einem weiteren Schritt erfolgte die Bestimmung der Mittelwertdifferenz zwischen altersstandardisierten Normwerten (WHOQOL-BREF) und Patientenmittelwerten (unsere Studie). Positive Werte indizierten eine höhere Zufriedenheit mit der Lebensqualität der jeweiligen Domäne, negative Werte indizierten eine geringere Zufriedenheit.

Dementsprechend erfolgte die Aufteilung der Patienten nach den oben genannten Kriterien (Geschlecht/Schulabschluss/Familienstand/Tumorentität). Für jede Gruppe wurde erneut die Berechnung der indirekt altersstandardisierten Normwerte des WHOQOL-BREF durchgeführt. Geschlechtsspezifische Mittelwerte der Domäne lagen für den WHOQOL-BREF bereits vor.

Die erhobenen Mittelwertdifferenzen zweier Gruppen (bspw. Männer und Frauen) konnten nun mittels t-Test auf ihre Signifikanz geprüft werden. Wurden 3 Gruppen verglichen (bspw. Melanom/nicht melanozytäre Tumoren/beide Tumorentitäten) kam ANOVA (analysis of variance) zum Einsatz.

Um die unterschiedlichen Altersgruppen miteinander zu vergleichen, war es notwendig, eine altersstratifizierte Auswertung (ohne Altersstandardisierung) durchzuführen. Hierbei wurden die Mittelwerte der einzelnen Altersgruppen mit den nicht standardisierten Normwerten aus dem Handbuch des WHOQOL-BREF verwendet. Dies ermöglichte Aussagen zum Vergleich einer bestimmten Altersgruppe (bspw. 26-35 Jahre) und derselben Altersgruppe der deutschen Allgemeinbevölkerung.

\subsubsection{Statistische Auswertung des Homburger Gesundheitsbogens}

Auch beim Homburger Gesundheitsbogen wurden die Patienten nach oben genannten Merkmalen (Geschlecht/Art des Tumors etc.) aufgeteilt und verglichen.

Der Vergleich zweier Merkmale (z.B. geschlechtsspezifischer Vergleich) erfolgte, indem Fragen, die 
mittels Likert Skala beantwortet worden waren, mittels Mann Whitney U Test auf ihre Signifikanz geprüft wurden. Bei Fragen mit zwei Antwortmöglichkeiten (wie sie im letzten Teil des Homburger Gesundheitsbogens vorkommen), wurde mittels Fisher-Exakt Test auf Signifikanz geprüft.

Die Prüfung, ob sich die Ergebnisse je nach Tumorart des Patienten signifikant unterschieden, erfolgte im ersten Teil (Fragen mit Likert Skala) mit dem Kruskal-Wallis-Test. Im zweiten Teil (zwei Antwortmöglichkeiten) fand der Chi-Quadrat-Test (Pearson-Chi-Quadrat-Test) Anwendung.

Eine Besonderheit stellte die Signifikanzprüfung der Frage nach der Regelmäßigkeit und Frequenz von Vorsorgeuntersuchungen (vor wie nach der Diagnose der Hauttumorerkrankung) dar. Ob Patienten beispielsweise nach der Diagnose ihrer Tumorerkrankung signifikant häufiger zum Hautarzt gingen als zuvor wurde mittels McNemar Test überprüft. 


\section{Ergebnisse}

\subsection{Patientenübersicht}

\subsubsection{Gesamtheit und Rücklaufquote}

Insgesamt wurden 653 Patienten angeschrieben. Davon waren 61,7\% männlich und 38,3\% weiblich. Es antworteten 263 Patienten. Somit betrug die Rücklaufquote rund 40,3\%.

163 der antwortenden Patienten waren männlich (62\%) und 100 weiblich (38\%). Die Rücklaufquote zeigte sich sowohl bei den Männern als auch bei den Frauen mit etwa $40 \%$ nahezu identisch (Abbildung 4.1). Insgesamt konnten 20 Patienten nicht erreicht werden. 12 Patienten hatten ihre Adresse geändert (Brief konnte nicht zugestellt werden); 8 Patienten waren zum Befragungszeitpunkt verstorben.

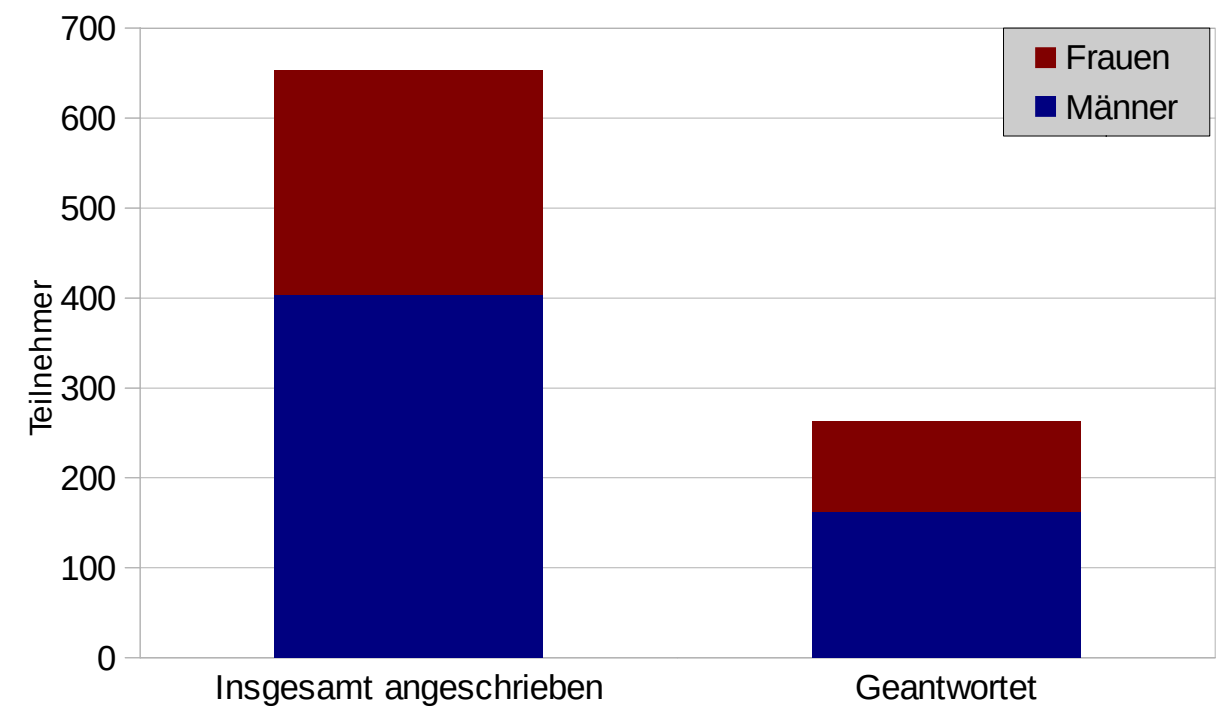

Abbildung 4.1: Patientenübersicht und Rücklaufquote

\subsubsection{Altersverteilung}

Der Altersdurchschnitt der Patienten, die den Fragebogen beantwortet hatten, lag zum Stichdatum am 01.09.2014 bei 69,8 Jahren. Die Patientinnen waren im Mittel mit 64,36 Jahren jünger als die männlichen Patienten mit 73,12 Jahren.

57,5\% ( $n=176)$ der Patienten waren zwischen 66 und 85 Jahren alt. 33,1\% ( $n=87)$ waren jünger als 65 Jahre. Die Frauen waren mit einem Altersdurchschnitt von 64,36 Jahren etwas jünger als die Männer (73,12 Jahre). Eine genaue Altersverteilung, sowie eine Unterteilung nach Geschlecht, 
findet sich in Abbildung 4.2.

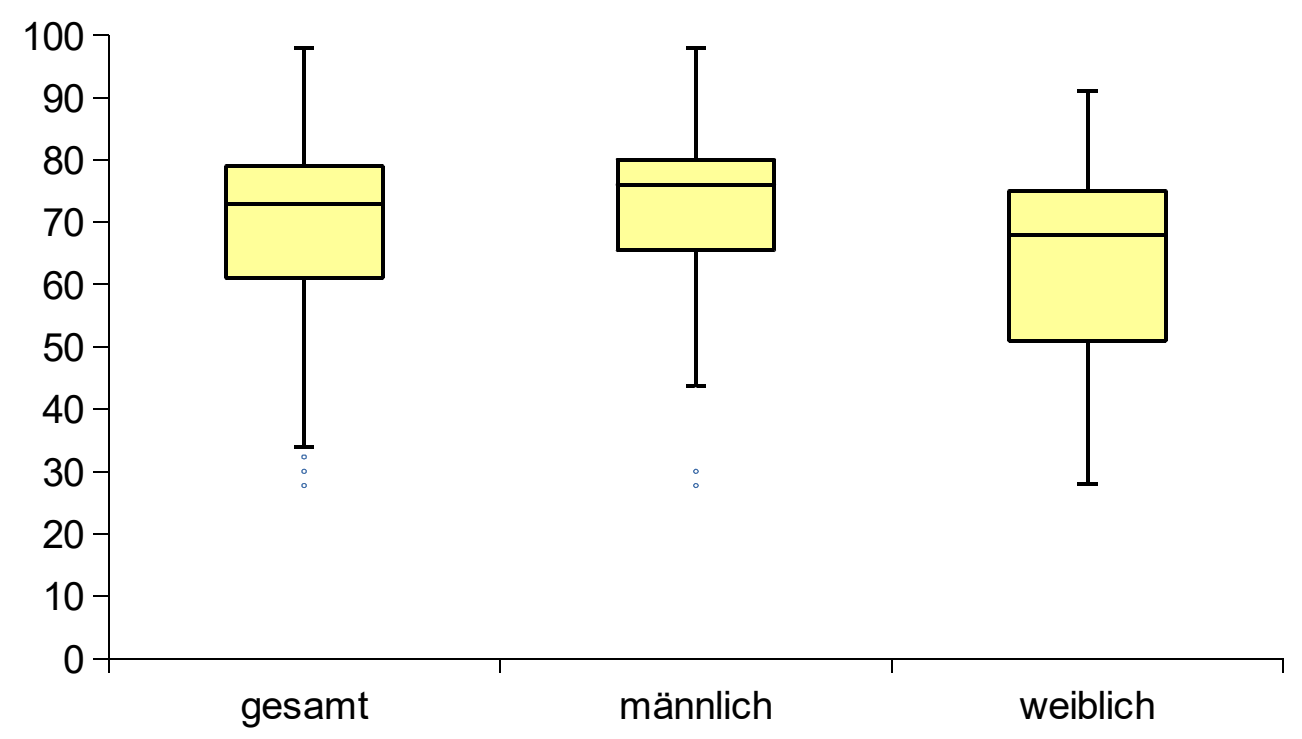

\section{Abbildung 4.2: Boxplot - Altersverteilung}

\subsubsection{Tumorentität}

In der Aufteilung nach der Grunderkrankung zeigte sich, dass 30\% ( $\mathrm{n}=79)$ der Patienten einen melanozytären Tumor hatten und 53,6\% (n=141) unter einem nicht-melanozytären Tumor litten. 16,3\% ( $n=43)$ der Patienten hatten sowohl eine melanozytäre als auch eine nicht-melanozytäre Tumorerkrankung (Abbildung 4.3).

Der Altersdurchschnitt der Patienten mit Melanomen lag bei 63,16 Jahren bzw. bei 73,67 Jahren bei denen mit nicht- melanozytären Tumoren. Der Altersdurchschnitt der Patienten mit beiden Tumorentitäten lag bei 69,26 Jahren.

Bei den Frauen litten 40,2\% (n=39; Altersdurchschnitt: 57,65 Jahre), bei den Männern 22,7\% ( $\mathrm{n}=35$; Altersdurchschnitt: 68,82 Jahre) unter einem melanozytären Tumor. 57,8\% (n=89; Altersdurchschnitt: 75,74) der Männer und 47,4\% (n=46; Altersdurchschnitt: 69,51 Jahre) der Frauen hatten einen nicht-melanozytären Tumor. Mit 19,5\% (n=30; Altersdurchschnitt 70,5 Jahre) bei den Männern und 12,4\% ( $n=12$; Altersdurchschnitt: 66,38) bei den Frauen litt der geringste Anteil bei beiden Geschlechtern unter beiden Tumorentitäten (Abbildung 4.4). 


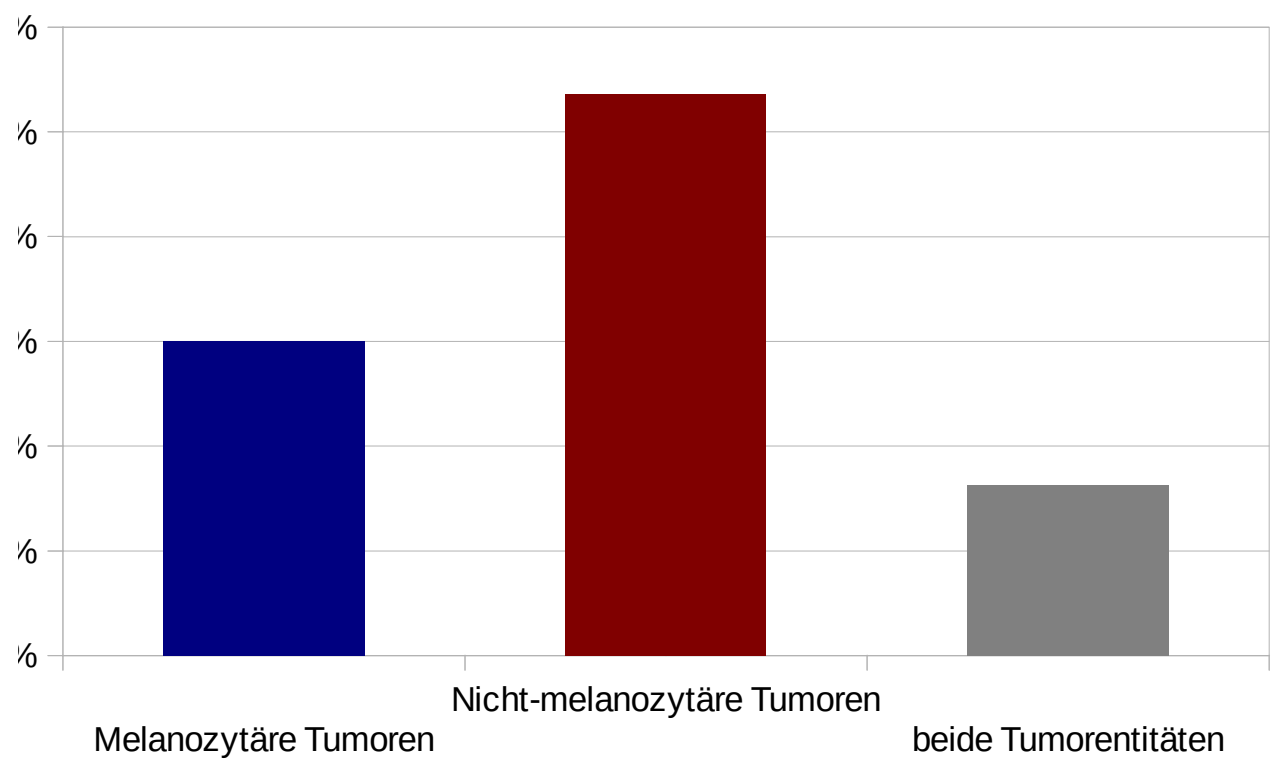

Abbildung 4.3: Tumorentitäten

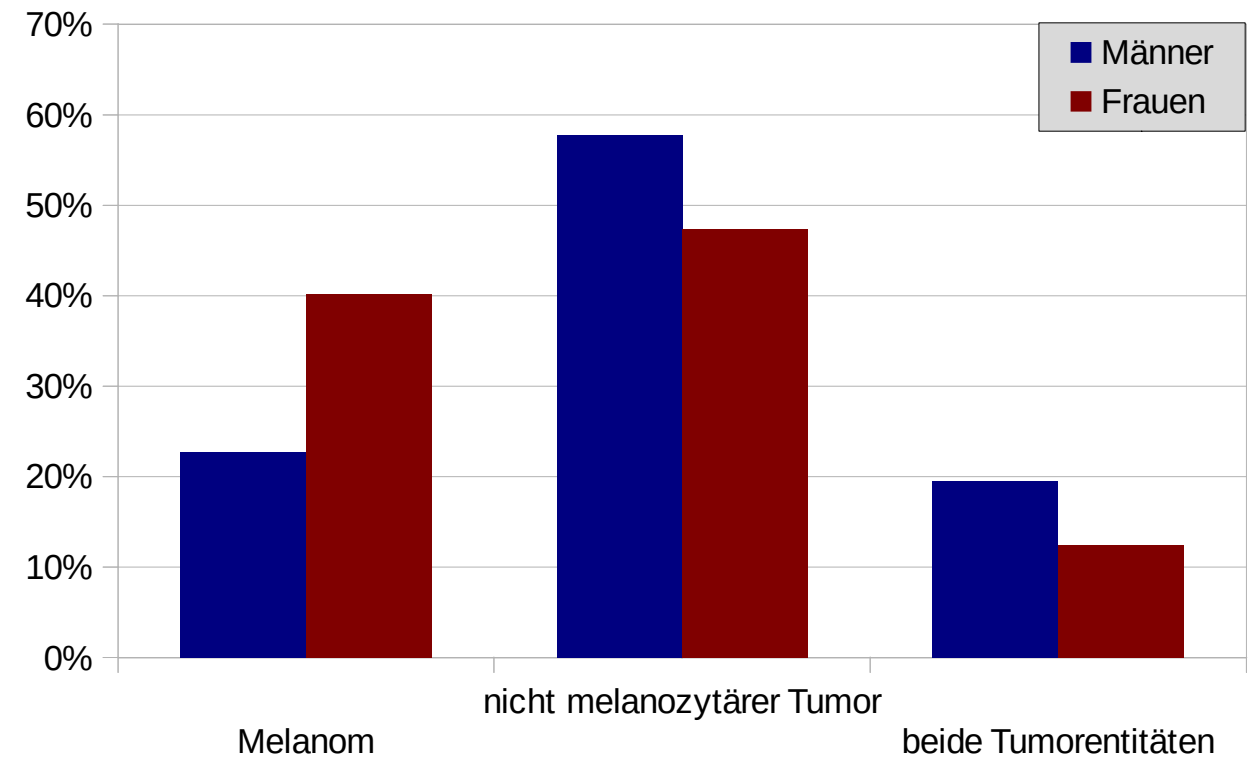

Abbildung 4.4: Tumorentitäten - geschlechtsspezifisch

\subsubsection{Qualifikation}

Bei allen befragten Patienten wurde der jeweils höchste erreichte Abschluss ermittelt. 4 Patienten beantworteten die Frage nicht. Unter den antwortenden Patienten hatten 76,4\% (n=198) einen relativ niedrigen Qualifikationsgrad (kein Abschluss, Hauptschulabschluss oder mittlere Reife). Davon hatten 1,5\% ( $n=4)$ keinen Abschluss, 54,4\% $(n=141)$ hatten einen Hauptschulabschluss und 20,5\% ( $n=53)$ als höchste Qualifikation die mittlere Reife. Insgesamt 23,5\% ( $n=61)$ hatten einen 
höheren Qualifikationsgrad als die mittlere Reife, davon 5,7\% ( $\mathrm{n}=15)$ eine Fachhochschulreife, 2,3\% (n=6) das Abitur, 8,5\% (n=22) einen Fachhochschulabschluss, 5,4\% (n=14) einen universitären Abschluss und 1,5\% $(\mathrm{n}=4)$ waren postgraduiert (Doktor-Titel, Professur etc.). Eine graphische Darstellung findet sich in Abbildung 4.5.

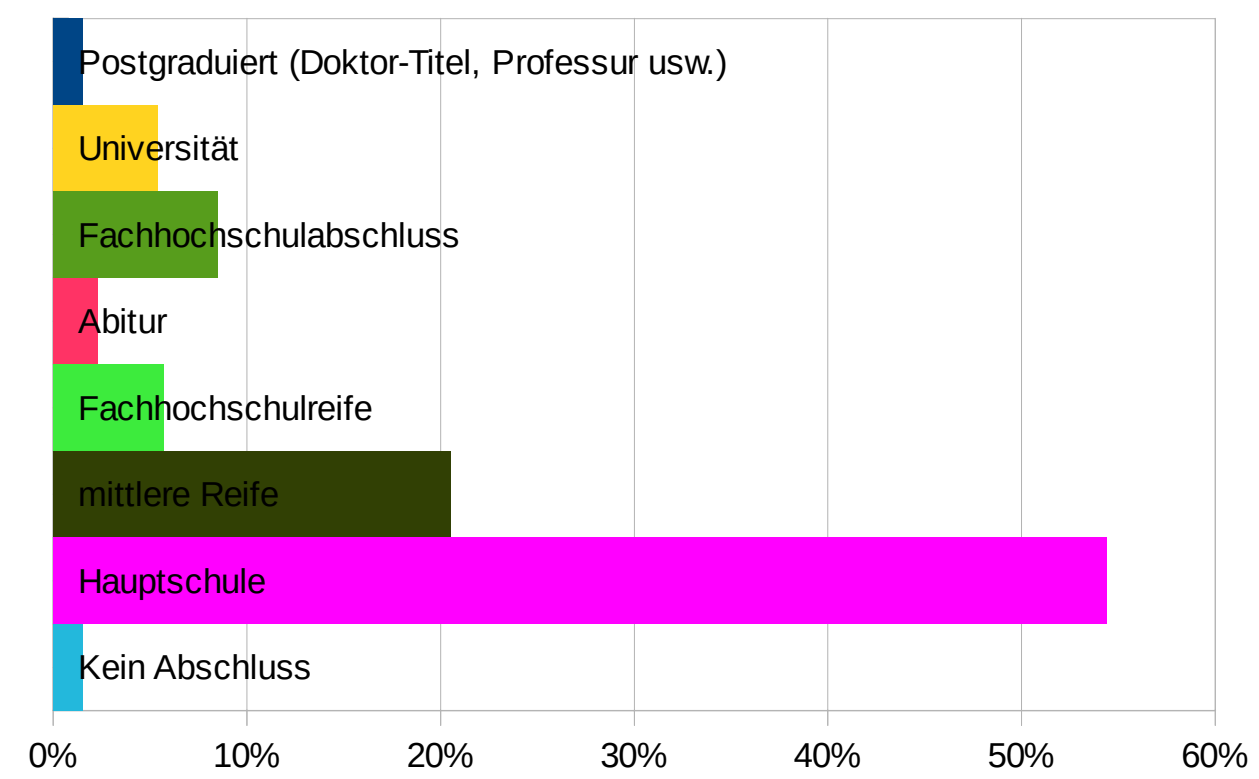

Abbildung 4.5: Qualifikation

In der geschlechtsspezifischen Betrachtung fanden sich bei den Männern 1,2\% (n=2) ohne Abschluss, 51,6\% ( $\mathrm{n}=83)$ mit Hauptschulabschluss, 17,4\% ( $\mathrm{n}=28)$ mit mittlerer Reife, 6,8\% ( $\mathrm{n}=11)$ mit Fachhochschulreife, 1,9\% (n=3) mit Abitur, 11,8\% ( $n=19)$ mit Fachhochschulabschluss und 6,8\% ( $n=11)$ mit einem Universitätsabschluss als höchste Qualifikation. 2,5\% ( $n=4)$ waren postgraduiert. 2 männliche Patienten beantworteten die Frage nicht.

Bei den Frauen hatten 2,0\% (n=2) keinen Abschluss. 59,2\% (n=58) hatten einen Hauptschulabschluss, 25,5\% $(n=25)$ die mittlere Reife, 4,1\% $(n=4)$ eine Fachhochschulreife, 3,1 $(n=3)$ das Abitur, 3,1\% (n=3) einen Fachhochschulabschluss und 3,1\% (n=3) einen Universitätsabschluss. Keine Patientin war postgraduiert. 2 Patientinnen beantworteten die Frage nach dem Schulabschluss nicht.

Zur besseren Übersicht wurden die Patienten beider Gruppen nun entsprechend niedrigerer Qualifikation (bis maximal mittlere Reife), und höherer Qualifikation (ab Fachhochschulreife) zusammengefasst. Eine graphische Darstellung hierzu findet sich in Abbildung 4.6. Es zeigte sich, dass die Männer mit insgesamt 29,8\% (n=48) höher qualifiziert waren als die Frauen mit 13,4\% (n=13). 


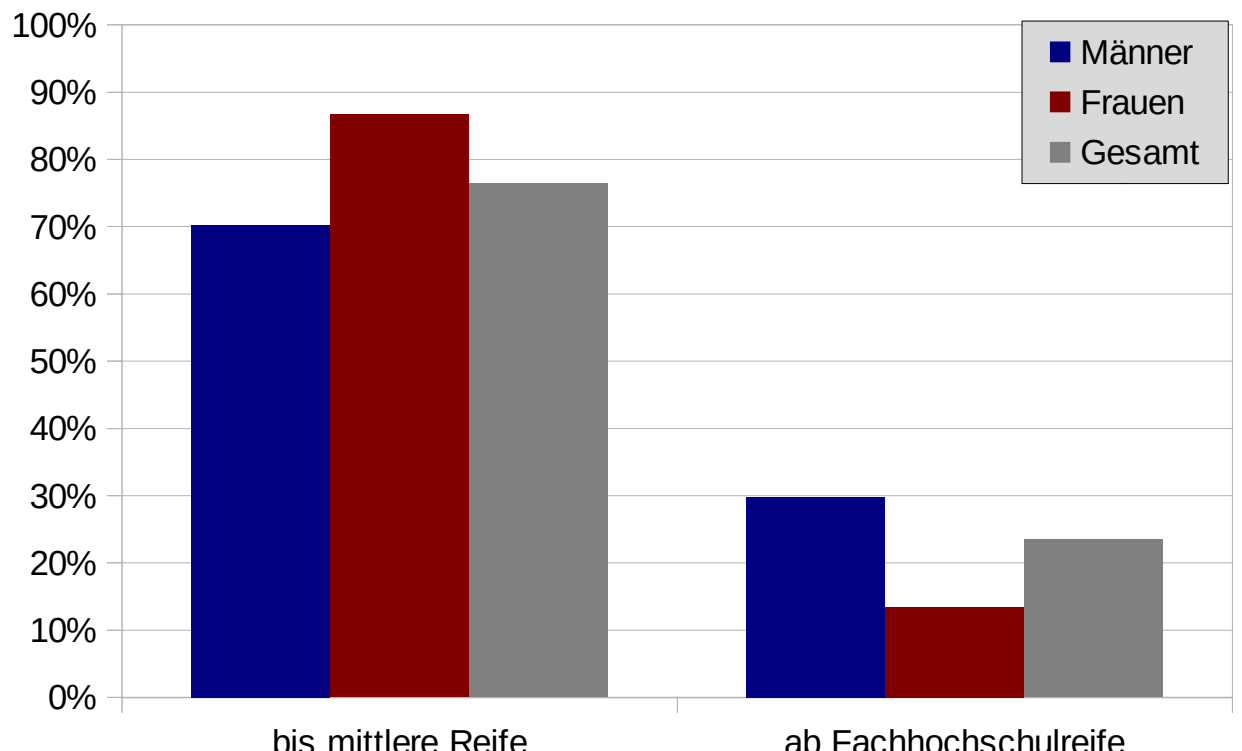

Abbildung 4.6: Qualifikation - geschlechtsspezifisch

\subsubsection{Familienstand}

Unter den Patienten, die die Frage nach dem Familienstand beantwortet hatten, waren mit 67,3\% $(n=175)$ die meisten verheiratet. 13,8\% $(n=36)$ waren verwitwet. 8,1\% $(n=21)$ lebten mit einem Partner, 7,7\% $(n=20)$ lebten allein, 0,4\% $(n=1)$ lebten getrennt und 2,7\% $(n=7)$ waren geschieden. 3 Patienten beantworteten die Frage nicht (Abbildung 4.7).

Unter den Männern gaben 5,6\% $(n=9)$ an allein zu leben, 74,1\% $(n=120)$ waren verheiratet, 9,3\% $(n=15)$ lebten mit einem Partner, 0,6\% $(n=1)$ lebten getrennt, 1,2\% $(n=2)$ waren geschieden und 9,3\% (n=15) waren verwitwet. Ein männlicher Patient beantwortete die Frage nicht.

Bei den Frauen waren 56,1\% $(n=55)$ verheiratet, 21,4\% $(n=21)$ verwitwet, 6,1\% $(n=6)$ lebten mit einem Partner, 11,2\% ( $\mathrm{n}=11)$ allein und 5,1\% $(\mathrm{n}=5)$ waren geschieden. Keine der Frauen gab an getrennt zu leben. 2 Patientinnen beantworteten die Frage nicht.

In Abbildung 4.8 wurden die Patienten danach aufgeteilt, ob sie mit oder ohne Partner lebten. Es zeigt sich das 37,7\% (n=37) der Frauen und 16,7\% (n=27) der Männer ohne Partner lebten. 


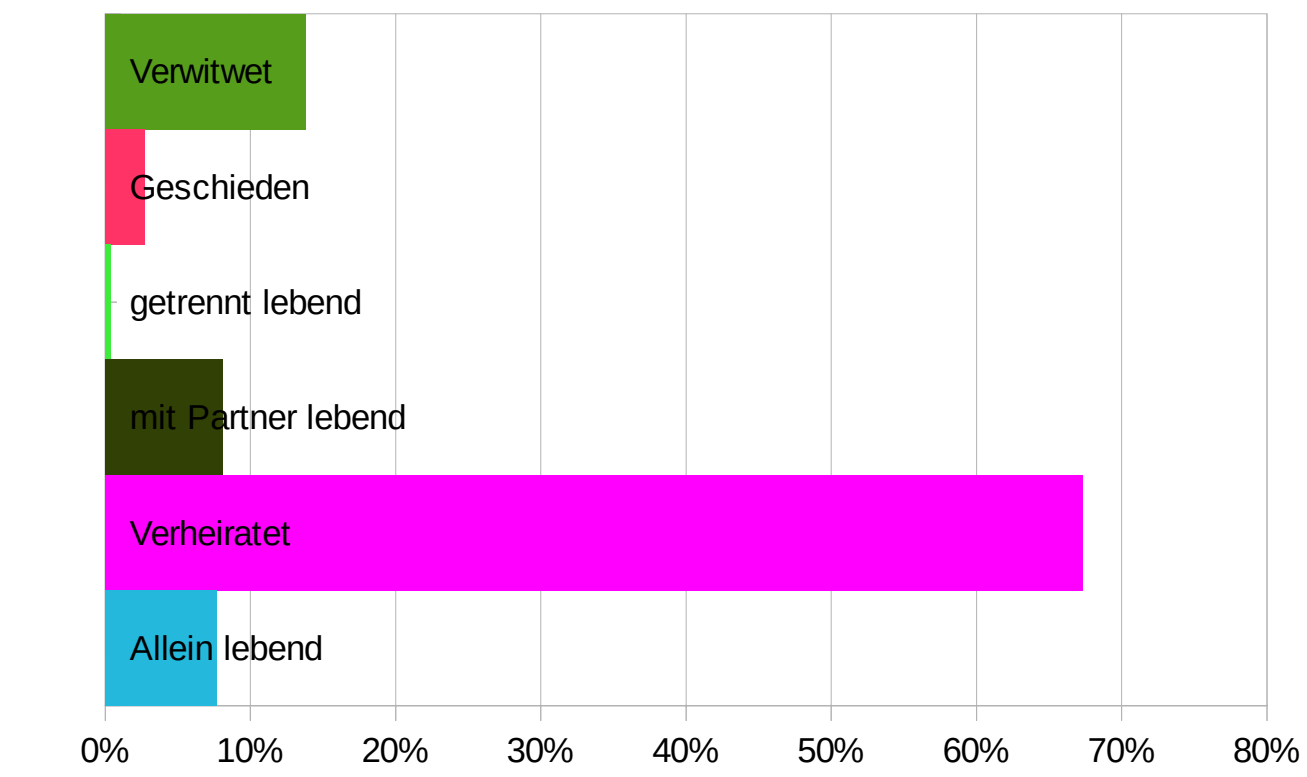

Abbildung 4.7: Familienstand

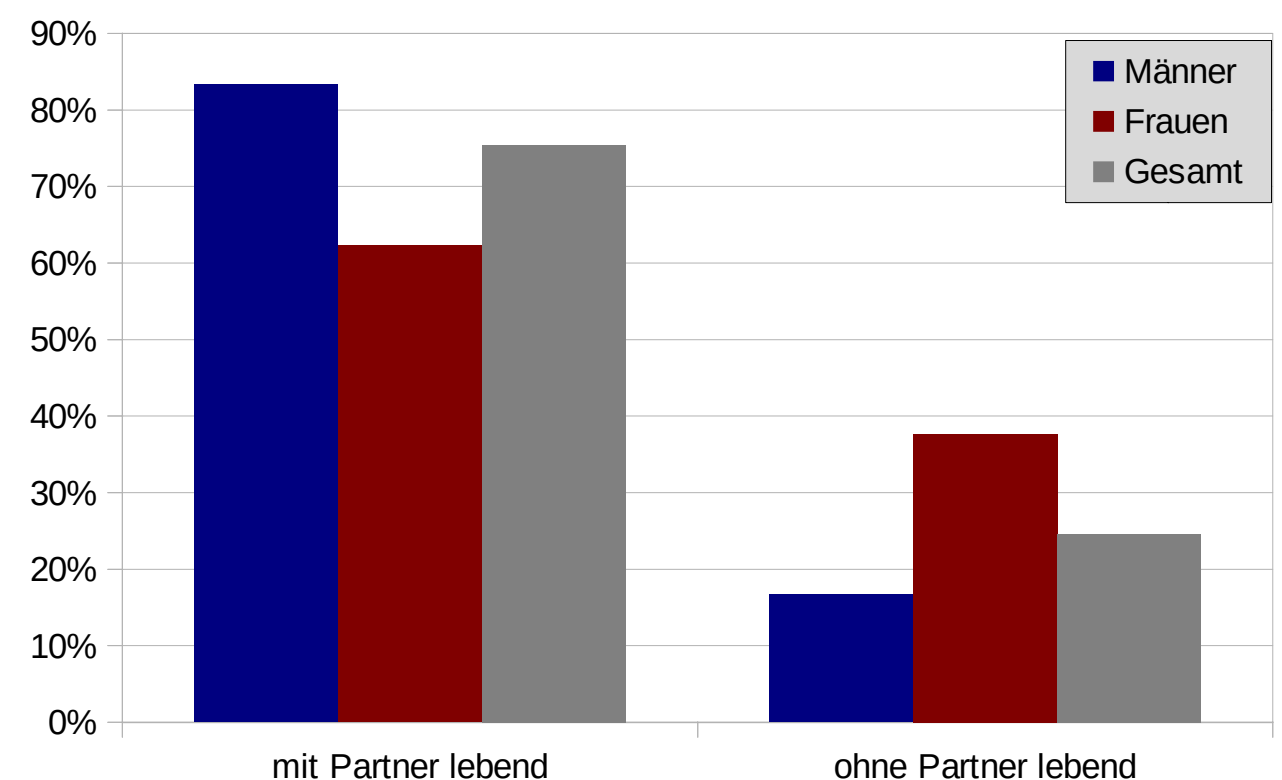

Abbildung 4.8: Familienstand - geschlechtsspezifisch 


\subsection{Ergebnisse des WHOQOL-BREF Fragebogens}

\subsubsection{Gesamtheit aller Patienten}

Die Auswertung des WHOQOL-BREF Fragebogens ergab in allen Domänen eine höhere Zufriedenheit der Patienten dieser Studie im Vergleich zu den altersstandardisierten Normwerten des WHOQOL-BREF Fragebogens. Eine genaue Aufstellung findet sich in Tabelle 4.1 und in Abbildung 4.9. In den Domänen „Psychisch“, „soziale Beziehung“ und insbesondere „Umwelt“ war der Unterschied besonders groß und es zeigte sich eine statistische Signifikanz.

\section{Zur Erläuterung (siehe auch Material und Methoden):}

Die Mittelwertdifferenzen ergaben sich aus dem Unterschied zwischen dem altersstandardisierten Normwert des WHOQOL-BREF und unserem Patientenmittelwert. Ersterer wurde von letzterem abgezogen. Positive Werte indizierten eine höhere Zufriedenheit mit der Lebensqualität in der jeweiligen Domäne, negative Werte indizierten eine geringere Zufriedenheit seitens der Patienten unserer Studie im Vergleich zu den Normwerten des WHOQOL-BREF.

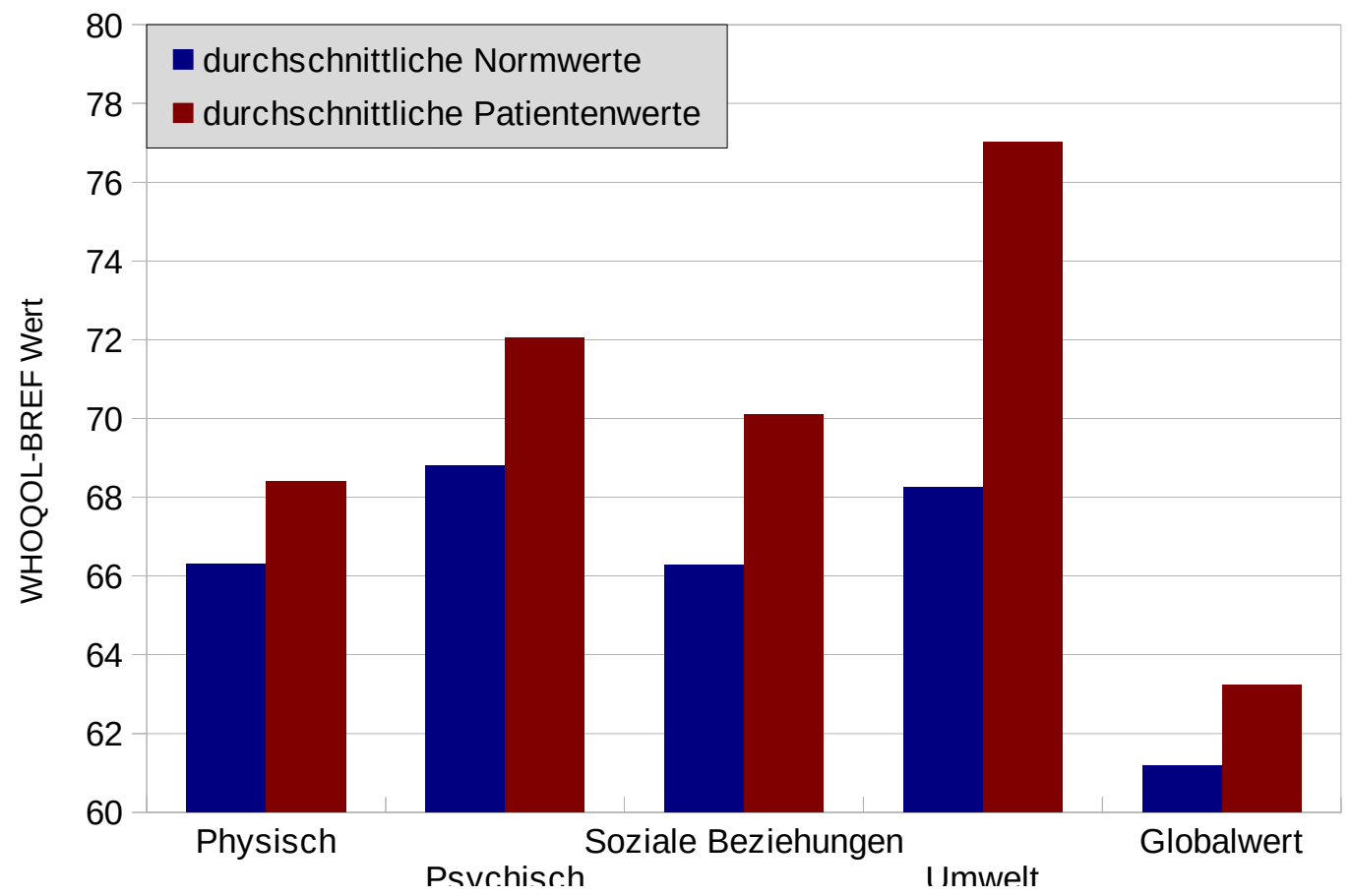

Abbildung 4.9: Vergleich der Lebensqualität aller Patienten mit den altersstandardisierten Normwerten 


\begin{tabular}{|l|c|c|c|c|}
\hline & $\begin{array}{c}\text { altersstandardisierte } \\
\text { Normwerte }\end{array}$ & Patientenmittelwerte & Mittelwertdifferenz & p \\
\hline Physisch & 66,31 & 68,40 & 2,09 & 0,084 \\
\hline Psychisch & 68,81 & 72,05 & 3,24 & $\mathbf{0 , 0 0 1}$ \\
\hline Soziale Beziehung & 66,27 & 70,09 & 3,82 & $<\mathbf{0 , 0 0 1}$ \\
\hline Umwelt & 68,25 & 77,01 & 8,76 & $<\mathbf{0 , 0 0 1}$ \\
\hline Globalwert & 61,18 & 63,23 & 2,05 & 0,081 \\
\hline
\end{tabular}

Tabelle 4.1: Vergleich der Lebensqualität aller Patienten mit den altersstandardisierten Normwerten

\subsubsection{Geschlechtsspezifische Auswertung}

Um herauszufinden, ob es einen Unterschied in der Lebensqualität zwischen Männern und Frauen gab, wurden in der Aufteilung nach Männern und Frauen die in dieser Studie erhobenen Werte mit den altersstandardisierten und geschlechtsspezifischen Normwerten des WHOQOL-BREF verglichen.

\subsubsection{Männer}

Bei den Männern zeigte sich in den Domänen „Physisch“, „Psychisch“, „Soziale Beziehung“ und „Umwelt“ eine positive Mittelwertdifferenz. Im Vergleich zu den altersstandardisierten Normwerten für Männer aus dem WHOQOL-BREF sprach dies zwar durchaus für eine höhere Zufriedenheit mit der Lebensqualität, allerdings in geringerem Maße als bei der Gesamtheit aller Patienten. Im Vergleich zu den altersstandardisierten Normwerten des WHOQOL-BREF zeigte sich die höhere Zufriedenheit in der Domäne „Umwelt“ signifikant. Beim „Globalwert“ zeigte sich eine negative Mittelwertdifferenz, was bei den Männern auf eine schlechtere Empfindung des allgemeinen Gesundheitszustandes und eine geringere Zufriedenheit mit dem Gesundheitszustand hinwies (Tabelle 4.2 und Abbildung 4.10).

\begin{tabular}{|l|c|c|c|c|}
\hline & $\begin{array}{c}\text { altersstandardisierte } \\
\text { Normwerte }\end{array}$ & Patientenmittelwerte & Mittelwertdifferenz & $\mathrm{p}$ \\
\hline Physisch & 65,16 & 65,75 & 0,59 & 0,700 \\
\hline Psychisch & 69,74 & 71,45 & 1,71 & 0,160 \\
\hline Soziale Beziehung & 65,05 & 67,26 & 2,21 & 0,094 \\
\hline Umwelt & 70,71 & 77,14 & 6,43 & $<\mathbf{0 , 0 0 1}$ \\
\hline Globalwert & 61.80 & 61,65 & $-0,15$ & 0,919 \\
\hline
\end{tabular}

Tabelle 4.2: Vergleich der Lebensqualität von Männern mit den altersstandardisierten Normwerten 


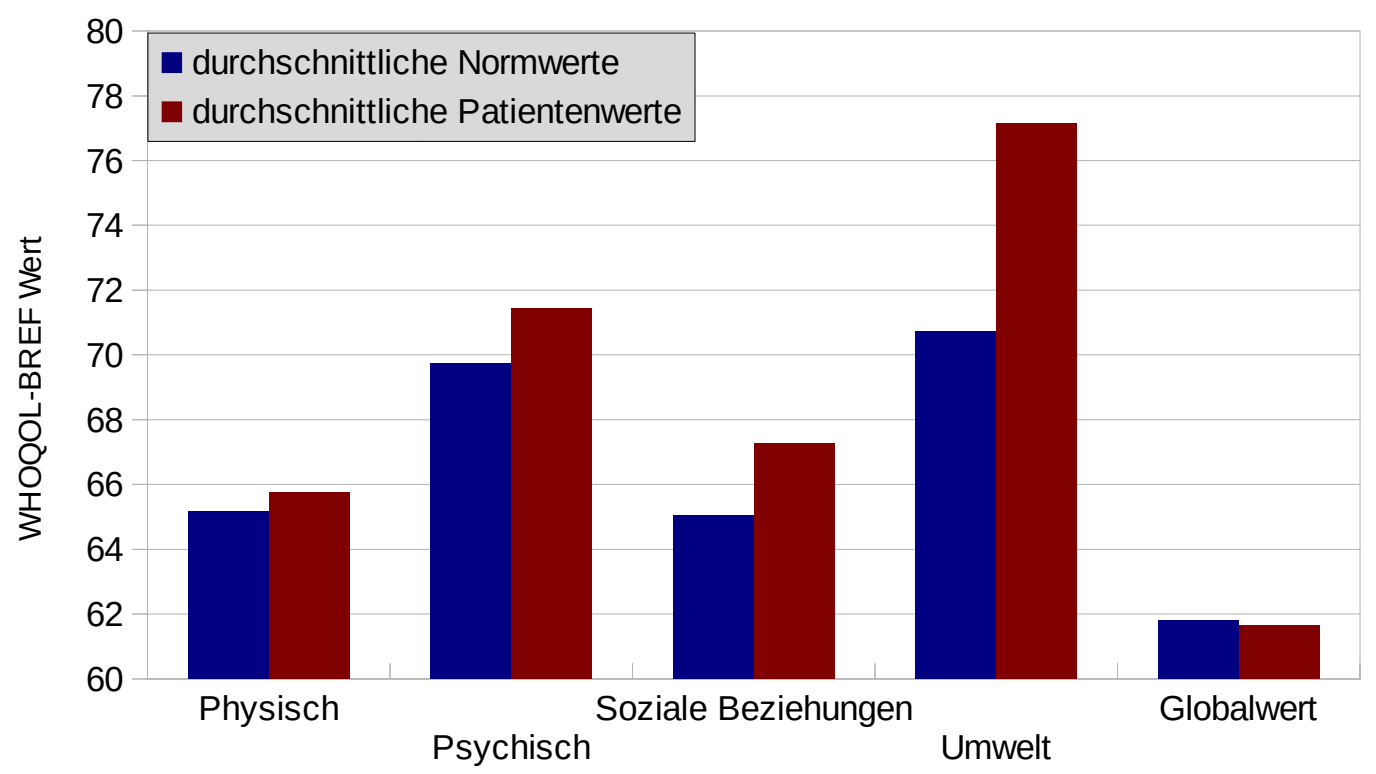

Abbildung 4.10: Vergleich der Lebensqualität von Männern mit den altersstandardisierten Normwerten

\subsubsection{Frauen}

Bei den Frauen zeigte sich in allen Domänen eine positive Mittelwertdifferenz im Vergleich zu den altersstandardisierten Normwerten. Diese fiel in allen Domänen höher aus als dies bei der Gesamtheit aller Patienten war. Im Vergleich $\mathrm{zu}$ den altersstandardisierten und geschlechtsspezifischen Normwerten (Tabelle 4.3 und Abbildung 4.11) zeigte sich in den Domänen „Physisch“, „Psychisch“, „Soziale Beziehung“ und „Umwelt“ eine statistische Signifikanz.

\begin{tabular}{|l|c|c|c|c|}
\hline & $\begin{array}{c}\text { altersstandardisierte } \\
\text { Normwerte }\end{array}$ & Patientenmittelwerte & Mittelwertdifferenz & $\mathrm{p}$ \\
\hline Physisch & 68,91 & 72,88 & 3,97 & $\mathbf{0 , 0 3 3}$ \\
\hline Psychisch & 69,08 & 73,06 & 3,98 & $\mathbf{0 , 0 1 1}$ \\
\hline Soziale Beziehung & 67,57 & 74,87 & 7,30 & $<\mathbf{0 , 0 0 1}$ \\
\hline Umwelt & 67,87 & 76,79 & 8,92 & $<\mathbf{0 , 0 0 1}$ \\
\hline Globalwert & 62,20 & 65,89 & 3,69 & 0,058 \\
\hline
\end{tabular}

Tabelle 4.3: Vergleich der Lebensqualität von Frauen mit den altersstandardisierten Normwerten 


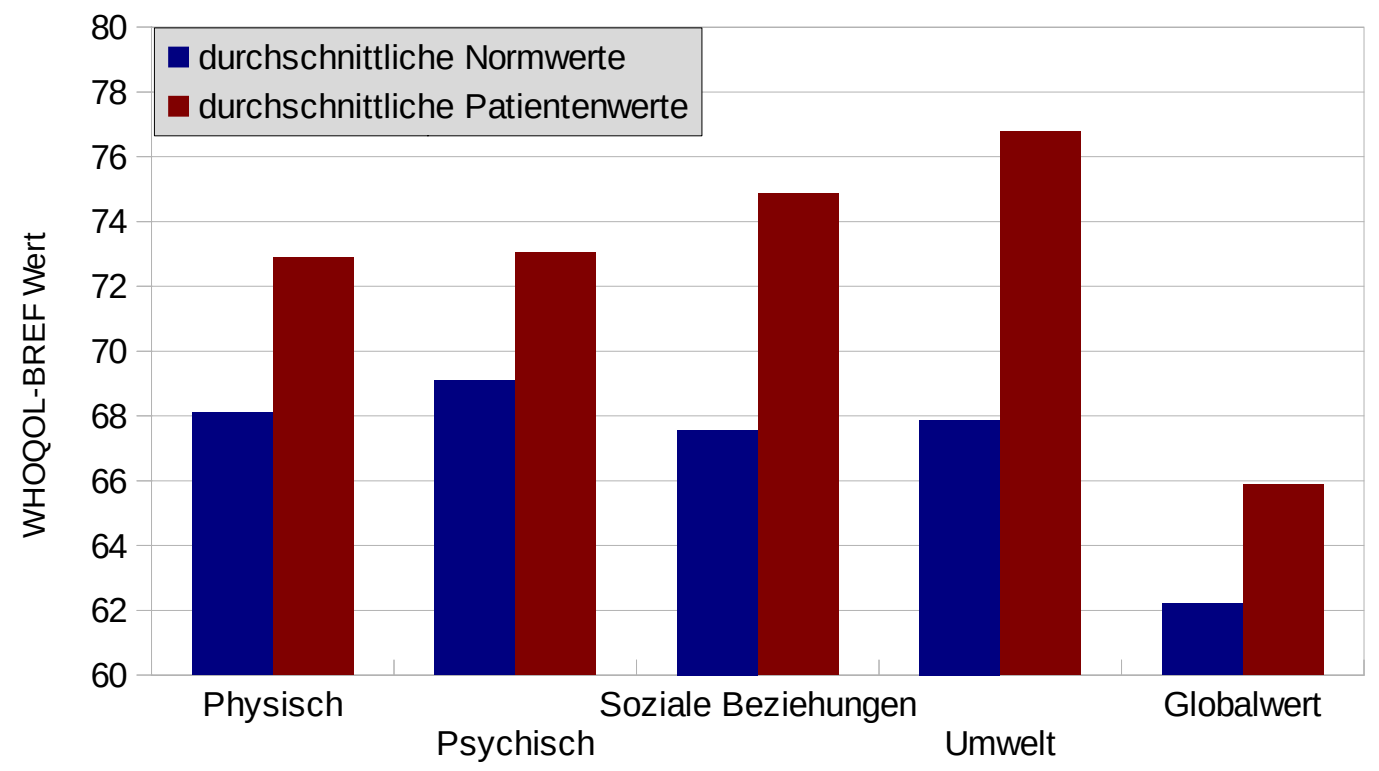

Abbildung 4.11: Vergleich der Lebensqualität von Frauen mit den altersstandardisierten Normwerten

\subsubsection{Vergleich der Unterschiede}

Im Vergleich zwischen Männern und Frauen zeigte sich, dass die befragten Frauen in allen Domänen zufriedener mit ihrer Lebensqualität waren als dies bei den Männern der Fall war (Tabelle 4.4). Allerdings zeigte sich der Unterschied nur in der Domäne „Soziale Beziehung“ signifikant.

\begin{tabular}{|l|c|c|c|}
\hline & $\begin{array}{c}\text { Mittelwertdifferenz } \\
\text { Männer }\end{array}$ & $\begin{array}{c}\text { Mittelwertdifferenz } \\
\text { Frauen }\end{array}$ & $\mathrm{p}$ \\
\hline Physisch & 0,59 & 3,97 & 0,170 \\
\hline Psychisch & 1,71 & 3,98 & 0,251 \\
\hline Soziale Beziehung & 2,21 & 7,30 & $\mathbf{0 , 0 1 9}$ \\
\hline Umwelt & 6,44 & 8,92 & 0,154 \\
\hline Globalwert & $-0,15$ & 3,69 & 0,113 \\
\hline
\end{tabular}

Tabelle 4.4: Vergleich der Lebensqualität von Männern und Frauen

\subsubsection{Altersspezifische Auswertung}

Nachfolgend wurden die verschiedenen Altersgruppen getrennt betrachtet und mit den im WHOQOL-BREF Handbuch angegebenen Normwerten für die deutsche Allgemeinbevölkerung derselben Altersgruppe (also nicht altersstandardisiert) verglichen. Auf diese Weise sollte untersucht werden, ob es in den Altersgruppen Unterschiede in Bezug auf die Lebensqualität gab. 


\subsubsection{Altersgruppe - 26-35 Jahre}

In der Gruppe der 26-35 jährigen gab es insgesamt nur 6 Patienten. In den Domänen „Physisch“, „Psychisch“, „Soziale Beziehung“ und „Umwelt“ wiesen diese eine positive Mittelwertdifferenz auf, was für eine höhere Zufriedenheit mit der Lebensqualität in diesen Domänen sprach. Beim „Globalwert“ ergab sich eine negative Mittelwertdifferenz, was wiederum auf eine geringere Zufriedenheit, u.a. in Bezug auf den allgemeinen Gesundheitszustand, hinwies. Mit Ausnahme der Domäne „Umwelt“ war keiner der Unterschiede signifikant (Tabelle 4.5).

\begin{tabular}{|l|c|c|c|c|}
\hline & Normwerte & Patientenmittelwerte & Mittelwertdifferenz & $\mathrm{p}$ \\
\hline Physisch & 84,11 & 86,90 & 2,79 & 0,552 \\
\hline Psychisch & 77,63 & 79,17 & 1,54 & 0,803 \\
\hline Soziale Beziehung & 74,83 & 79,17 & 4,34 & 0,677 \\
\hline Umwelt & 71,47 & 85,42 & 13,95 & $\mathbf{0 , 0 0 5}$ \\
\hline Globalwert & 73,29 & 64,58 & $-8,71$ & 0,256 \\
\hline
\end{tabular}

Tabelle 4.5: Vergleich der Lebensqualität von 26-35 jährigen mit den Normwerten

\subsubsection{Altersgruppe - 36-45 Jahre}

In dieser Gruppe gab es 8 Patienten. Sie wiesen in allen Domänen negative Mittelwertdifferenzen auf und waren somit unzufriedener mit der Lebensqualität als die deutsche Allgemeinbevölkerung derselben Altersgruppe. In den Domänen „Physisch“ und „Psychisch“ war der Unterschied besonders groß, sodass sich auch eine statistische Signifikanz ergab (Tabelle 4.6).

\begin{tabular}{|l|c|c|c|c|}
\hline & Normwerte & Patientenmittelwerte & Mittelwertdifferenz & p \\
\hline Physisch & 80,79 & 65,63 & $-15,16$ & $\mathbf{0 , 0 4 0}$ \\
\hline Psychisch & 74,19 & 64,06 & $-10,13$ & $\mathbf{0 , 0 0 7}$ \\
\hline Soziale Beziehung & 72,70 & 70,83 & $-1,87$ & 0,803 \\
\hline Umwelt & 70,05 & 67,97 & $-2,08$ & 0,431 \\
\hline Globalwert & 68,92 & 54,69 & $-14,23$ & 0,070 \\
\hline
\end{tabular}

Tabelle 4.6: Vergleich der Lebensqualität von 36-45 jährigen mit den Normwerten

\subsubsection{Altersgruppe - 46-55 Jahre}

In dieser Gruppe befanden sich 29 Patienten. Sie waren, bis auf die Domäne „Umwelt“, unzufriedener mit ihrer Lebensqualität, als dies für die Vergleichsgruppe (Normwerte des WHOQOL-BREF) der Fall war. In Bezug auf die Lebensqualität in der Domäne „Psychisch“ zeigte sich dies statistisch signifikant (Tabelle 4.7). 


\begin{tabular}{|l|c|c|c|c|}
\hline & Normwerte & Patientenmittelwerte & Mittelwertdifferenz & p \\
\hline Physisch & 76,85 & 70,07 & $-6,78$ & 0,051 \\
\hline Psychisch & 74,00 & 66,24 & $-7,76$ & $\mathbf{0 , 0 2 3}$ \\
\hline Soziale Beziehung & 71,49 & 66,95 & $-4,54$ & 0,202 \\
\hline Umwelt & 70,29 & 71,32 & 1,03 & 0,736 \\
\hline Globalwert & 66,31 & 60,34 & $-5,97$ & 0,125 \\
\hline
\end{tabular}

Tabelle 4.7: Vergleich der Lebensqualität von 46-55 jährigen mit den Normwerten

\subsubsection{Altersgruppe - 56-65 Jahre}

In dieser Altersgruppe gab es insgesamt 44 Patienten. Mit Ausnahme der Domäne „Physisch“ (Mittelwertdifferenz -0,38) zeigten sich in allen Domänen positive Mittelwertdifferenzen - dies sprach für eine höhere Zufriedenheit mit der Lebensqualität im Vergleich zur deutschen Allgemeinbevölkerung. Die Differenz fiel insbesondere in der Domäne „Umwelt“ hoch aus und zeigte sich dort auch signifikant (Tabelle 4.8).

\begin{tabular}{|l|c|c|c|c|}
\hline & Normwerte & Patientenmittelwerte & Mittelwertdifferenz & $\mathrm{p}$ \\
\hline Physisch & 72,94 & 72,56 & $-0,38$ & 0,885 \\
\hline Psychisch & 72,82 & 74,43 & 1,61 & 0,426 \\
\hline Soziale Beziehung & 70,08 & 72,82 & 2,74 & 0,266 \\
\hline Umwelt & 70,67 & 79,38 & 8,71 & $<\mathbf{0 , 0 0 1}$ \\
\hline Globalwert & 63,20 & 67,90 & 4,70 & 0,065 \\
\hline
\end{tabular}

Tabelle 4.8: Vergleich der Lebensqualität von 56-65 jährigen mit den Normwerten

\subsubsection{Altersgruppe - 66-75 Jahre}

In dieser Altersgruppe gab es 68 Patienten die in allen Domänen eine positive Mittelwertdifferenz aufwiesen. In der Domäne „Umwelt“ zeigte sich dieser Unterschied wiederum statistisch signifikant (Tabelle 4.9).

\begin{tabular}{|l|c|c|c|c|}
\hline & Normwerte & Patientenmittelwerte & Mittelwertdifferenz & $\mathrm{p}$ \\
\hline Physisch & 66,48 & 70,02 & 3,54 & 0,160 \\
\hline Psychisch & 70,53 & 74,24 & 3,71 & 0,059 \\
\hline Soziale Beziehung & 68,41 & 70,52 & 2,11 & 0,302 \\
\hline Umwelt & 70,20 & 79,11 & 8,91 & $<\mathbf{0 , 0 0 1}$ \\
\hline Globalwert & 62,13 & 65,15 & 3,02 & 0,244 \\
\hline
\end{tabular}

Tabelle 4.9: Vergleich der Lebensqualität von 66-75 jährigen mit den Normwerten 


\subsubsection{Altersgruppe - 76-85 Jahre}

Mit 83 Patienten war dies größte Gruppe. Auch hier zeigte sich in allen Domänen der Lebensqualität eine positive Mittelwertdifferenz und somit eine höhere Zufriedenheit mit der Lebensqualität als in der deutschen Allgemeinbevölkerung. Bis auf den „Globalwert“ ergaben die Unterschiede eine statistische Signifikanz (Tabelle 4.10).

\begin{tabular}{|l|c|c|c|c|}
\hline & Normwerte & Patientenmittelwerte & Mittelwertdifferenz & $\mathrm{p}$ \\
\hline Physisch & 60,18 & 64,87 & 4,69 & $\mathbf{0 , 0 3 3}$ \\
\hline Psychisch & 65,70 & 72,01 & 6,31 & $<\mathbf{0 , 0 0 1}$ \\
\hline Soziale Beziehung & 64,40 & 69,72 & 5,32 & $\mathbf{0 , 0 0 2}$ \\
\hline Umwelt & 67,30 & 76,57 & 9,27 & $<\mathbf{0 , 0 0 1}$ \\
\hline Globalwert & 58,58 & 61,73 & 3,15 & 0,077 \\
\hline
\end{tabular}

Tabelle 4.10: Vergleich der Lebensqualität von 76-85 jährigen mit den Normwerten

\subsubsection{Altersgruppe - älter als 85 Jahre}

In dieser Gruppe gab es 25 Patienten. Es zeigten sich noch höhere Mittelwertdifferenzen als in der Gruppe der 76-85 jährigen. Die Unterschiede waren wiederum in allen Domänen bis auf den „Globalwert“ signifikant (Tabelle 4.11).

\subsubsection{Vergleich der verschiedenen Altersgruppen}

Führte man die Mittelwertdifferenzen für die verschiedenen Altersgruppen in den unterschiedlichen Domänen zusammen, ergab sich folgendes Diagramm (Abbildung 4.12). In fast allen Dömanen ergab sich mit zunehmendem Alter eine höhere Zufriedenheit mit der Lebensqualität. Eine Ausnahme stellten die Patienten zwischen 26-35 Jahren dar. Bei ihnen zeigte sich eine höhere Zufriedenheit mit der Lebensqualität in allen Domänen als bei den 36-45 jährigen.

\begin{tabular}{|l|c|c|c|c|}
\hline & Normwerte & Patientenmittelwerte & Mittelwertdifferenz & p \\
\hline Physisch & 51,98 & 62,42 & 10,44 & $\mathbf{0 , 0 2 6}$ \\
\hline Psychisch & 56.60 & 69,57 & 12,97 & $\mathbf{0 , 0 0 3}$ \\
\hline Soziale Beziehung & 48,48 & 66,30 & 17,82 & $\mathbf{0 , 0 0 1}$ \\
\hline Umwelt & 57,74 & 76,23 & 18,49 & $<\mathbf{0 , 0 0 1}$ \\
\hline Globalwert & 52,08 & 60,42 & 8,34 & 0,076 \\
\hline
\end{tabular}

Tabelle 4.11: Vergleich der Lebensqualität von über 85 jährigen mit den Normwerten 


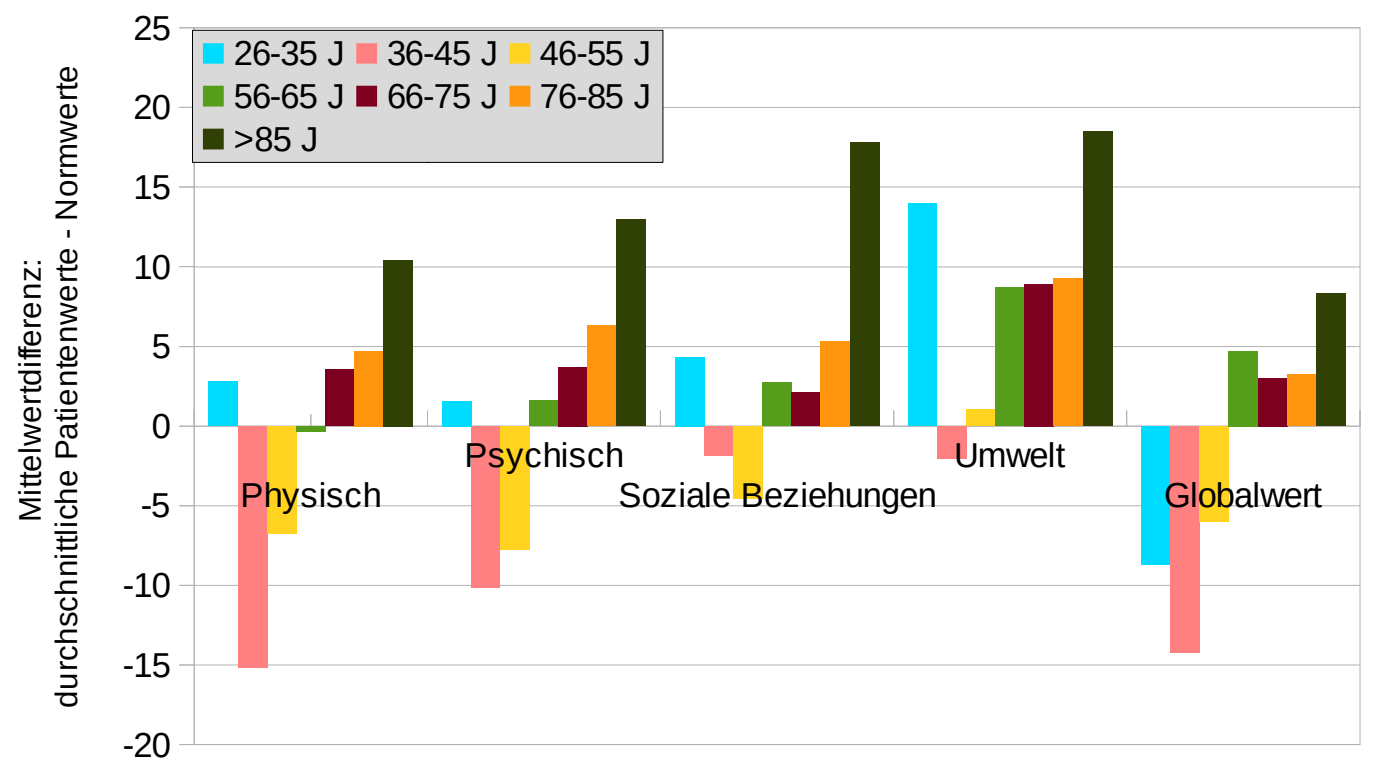

Abbildung 4.12: Vergleich der Lebensqualität gemäß der verschiedenen Altersgruppen

\subsubsection{Auswertung nach der Grunddiagnose}

In der folgenden Auswertung wurden die Patienten entsprechend ihrer dermatologischen Grunderkrankungen aufgeteilt. Ziel war die Beurteilung der Lebensqualität gemäß Tumorentität. Zu diesem Zweck erfolgte nun wieder der Vergleich mit den altersstandardisierten Normwerten.

\subsubsection{Patienten mit melanozytärem Tumor}

In dieser Gruppe gab es 79 Patienten. Im Vergleich zu den altersstandardisierten Normwerten zeigte sich in den Domänen „Physisch“, „Psychisch“, „Soziale Beziehung“ und „Umwelt“ eine positive Mittelwertdifferenz. Die Patienten waren vor allem mit ihrer Umwelt und ihren sozialen Beziehungen zufriedener als die deutsche Normalbevölkerung; hier zeigte sich der Unterschied statistisch signifikant (Tabelle 4.12). Die Mittelwertdifferenz für den Globalwert lag nur knapp im positiven Bereich.

\begin{tabular}{|l|c|c|c|c|}
\hline & $\begin{array}{c}\text { altersstandardisierte } \\
\text { Normwerte }\end{array}$ & Patientenmittelwerte & Mittelwertdifferenz & $\mathrm{p}$ \\
\hline Physisch & 69,87 & 71,07 & 1,20 & 0,570 \\
\hline Psychisch & 70,84 & 73,92 & 3,08 & 0,065 \\
\hline Soziale Beziehung & 68,40 & 72,63 & 4,23 & $\mathbf{0 , 0 3 9}$ \\
\hline Umwelt & 69,37 & 78,67 & 9,30 & $<\mathbf{0 , 0 0 1}$ \\
\hline Globalwert & 63,11 & 63,13 & 0,02 & 0,991 \\
\hline
\end{tabular}

Tabelle 4.12: Vergleich der Lebensqualität von Patienten mit Melanom mit den altersstandardisierten Normwerten 


\subsubsection{Patienten mit nicht melanozytärem Tumor}

Mit 141 Patienten war dies die größte Gruppe. Hier zeigten sich in allen Domänen positive Mittelwertdifferenzen und somit eine höhere Zufriedenheit mit der Lebensqualität als in der Vergleichsgruppe. In den Domänen „Psychisch“, „Soziale Beziehung“ und „Umwelt“ war dieser Unterschied signifikant. Auch in dieser Gruppe zeigten sich die Patienten vor allem mit ihrer Umwelt zufrieden (Tabelle 4.13).

\begin{tabular}{|l|c|c|c|c|}
\hline & $\begin{array}{c}\text { altersstandardisierte } \\
\text { Normwerte }\end{array}$ & Patientenmittelwerte & Mittelwertdifferenz & $\mathrm{p}$ \\
\hline Physisch & 64,03 & 66,93 & 2,90 & 0,080 \\
\hline Psychisch & 67,43 & 70,15 & 2,72 & $\mathbf{0 , 0 4 7}$ \\
\hline Soziale Beziehung & 64,76 & 68,17 & 3,41 & $\mathbf{0 , 0 1 9}$ \\
\hline Umwelt & 67,42 & 76,22 & 8,80 & $<\mathbf{0 , 0 0 1}$ \\
\hline Globalwert & 60,10 & 62,96 & 2,86 & 0,082 \\
\hline
\end{tabular}

Tabelle 4.13: Vergleich der Lebensqualität von Patienten mit nicht-melanozytärem Tumor mit den altersstandardisierten Normwerten

\subsubsection{Patienten mit beiden Tumorentitäten}

Insgesamt litten 43 Patienten unter beiden Tumorentitäten. Auch hier zeigte sich ein ähnliches Bild. Die Patienten waren in allen Domänen zufriedener als die deutsche Normalbevölkerung (positive Mittelwertdifferenz). In den Domänen „Psychisch“ und „Soziale Beziehung“ war der Unterschied statistisch signifikant (Tabelle 4.14).

\begin{tabular}{|l|c|c|c|c|}
\hline & $\begin{array}{c}\text { altersstandardisierte } \\
\text { Normwerte }\end{array}$ & Patientenmittelwerte & Mittelwertdifferenz & $\mathrm{p}$ \\
\hline Physisch & 66,88 & 68,16 & 1,28 & 0,692 \\
\hline Psychisch & 69,42 & 74,61 & 5,19 & $\mathbf{0 , 0 2 8}$ \\
\hline Soziale Beziehung & 67,11 & 71,51 & 4,40 & 0,092 \\
\hline Umwelt & 68,84 & 76,60 & 7,76 & $<\mathbf{0 , 0 0 1}$ \\
\hline Globalwert & 61,60 & 64,24 & 2,64 & 0,399 \\
\hline
\end{tabular}

Tabelle 4.14: Vergleich Lebensqualität von Patienten mit beiden Tumorentitäten und den altersstandardisierten Normwerten 


\subsubsection{Vergleich der Unterschiede}

Beim Vergleich aller Mittelwertdifferenzen der verschiedenen Gruppen ergaben sich keine signifikanten Unterschiede. Eine genaue Aufstellung findet sich in Tabelle 4.15.

\begin{tabular}{|l|c|c|c|c|}
\hline & $\begin{array}{c}\text { Mittelwertdifferenz } \\
\text { Melanom }\end{array}$ & $\begin{array}{c}\text { Mittelwertdifferenz } \\
\text { nicht melanozytärer } \\
\text { Tumor }\end{array}$ & $\begin{array}{c}\text { Mittelwertdifferenz } \\
\text { sowohl Melanom als } \\
\text { auch nicht } \\
\text { melanozytärer Tumor }\end{array}$ & p \\
\hline Physisch & 1,20 & 2,90 & 1,28 & 0,786 \\
\hline Psychisch & 3,08 & 2,72 & 5,19 & 0,649 \\
\hline Soziale Beziehung & 4,23 & 3,40 & 4,40 & 0,918 \\
\hline Umwelt & 9,31 & 8,80 & 7,76 & 0,834 \\
\hline Globalwert & 0,02 & 2,86 & 2,64 & 0,548 \\
\hline
\end{tabular}

Tabelle 4.15: Vergleich der Lebensqualität gemäß verschiedener Tumorentitäten

\subsubsection{Auswertung nach dem Schulabschluss}

Hier wurde geprüft ob es zwischen Lebensqualität und der höchsten erreichten Qualifikation des Patienten Zusammenhänge gab.

\subsubsection{Qualifikation: kein Abschluss, Hauptschule, mittlere Reife}

In dieser Gruppe befanden sich 198 Patienten. Es zeigten sich durchgehend positive Mittelwertdifferenzen im Vergleich zu den altersbezogenen Normwerten. Allerdings waren diese Mittelwertdifferenzen u.a. in den Domänen „Physisch“ und beim „Globalwert“ sehr niedrig. Eine statistische Signifikanz zeigte sich in den Domänen „Psychisch“, „Soziale Beziehung“ und „Umwelt“ (Tabelle 4.16).

\begin{tabular}{|l|c|c|c|c|}
\hline & $\begin{array}{c}\text { altersstandardisierte } \\
\text { Normwerte }\end{array}$ & Patientenmittelwerte & Mittelwertdifferenz & $\mathrm{p}$ \\
\hline Physisch & 66,14 & 66,88 & 0,74 & 0,596 \\
\hline Psychisch & 68,72 & 71,15 & 2,43 & $\mathbf{0 , 0 2 7}$ \\
\hline Soziale Beziehung & 66,14 & 70,09 & 3,95 & $\mathbf{0 , 0 0 2}$ \\
\hline Umwelt & 68,23 & 76,08 & 7,85 & $<\mathbf{0 , 0 0 1}$ \\
\hline Globalwert & 61,10 & 62,50 & 1,40 & 0,306 \\
\hline
\end{tabular}

Tabelle 4.16: Vergleich der Lebensqualität von Patienten mit geringerem Bildungsniveau mit den altersstandardisierten Normwerten 


\subsubsection{Qualifikation: Fachhochschulreife, Abitur, Fachhochschule, Universität, Postgraduiert}

Diese Gruppe beinhaltete 61 Patienten, die in allen Domänen ebenfalls positive Mittelwertdifferenzen aufwiesen. Dies entspricht einer höheren Lebensqualität als die der altersstandardisierten deutschen Normalbevölkerung. Die höhere Lebensqualität zeigte sich in den Domänen „Physisch“, Psychisch“ und „Umwelt“ statistisch signifikant (Tabelle 4.17).

\begin{tabular}{|l|c|c|c|c|}
\hline & $\begin{array}{c}\text { altersstandardisierte } \\
\text { Normwerte }\end{array}$ & Patientenmittelwerte & Mittelwertdifferenz & $\mathrm{p}$ \\
\hline Physisch & 66,99 & 73,20 & 6,21 & $\mathbf{0 , 0 1 3}$ \\
\hline Psychisch & 69,26 & 74,59 & 5,33 & $\mathbf{0 , 0 1 0}$ \\
\hline Soziale Beziehung & 66,97 & 69,99 & 3,02 & 0,158 \\
\hline Umwelt & 68,44 & 79,82 & 11,38 & $<\mathbf{0 , 0 0 1}$ \\
\hline Globalwert & 61,53 & 65,42 & 3,89 & 0,089 \\
\hline
\end{tabular}

Tabelle 4.17: Vergleich der Lebensqualität von Patienten mit höherem Bildungsniveau mit den altersstandardisierten Normwerten

\subsubsection{Vergleich der Unterschiede}

Im direkten Vergleich beider Gruppen stellte sich heraus, dass die Patienten mit höherer Qualifikation in den Domänen „Physisch“, „Psychisch“, „Umwelt“ und dem „Globalwert“ eine größere Zufriedenheit mit ihrer Lebensqualität aufwiesen. In der Domäne „Physisch“ war dieser Unterschied besonders groß. Umgekehrt war es bei der Domäne „Soziale Beziehung“. Hier waren die geringer qualifizierten Patienten geringfügig zufriedener als jene mit höherem Abschluss. Eine statistische Signifikanz lag in keiner der Domänen vor (Tabelle 4.18 und Abbildung 4.13).

\begin{tabular}{|l|c|c|c|}
\hline & $\begin{array}{c}\text { Mittelwertdifferenz } \\
\text { bis mittlere Reife }\end{array}$ & $\begin{array}{c}\text { Mittelwertdifferenz } \\
\text { ab Fachhochschule }\end{array}$ & $\mathrm{p}$ \\
\hline Physisch & 0,74 & 6,21 & 0,056 \\
\hline Psychisch & 2,43 & 5,33 & 0,205 \\
\hline Soziale Beziehung & 3,953 & 3,02 & 0,714 \\
\hline Umwelt & 7,85 & 11,38 & 0,075 \\
\hline Globalwert & 1,40 & 3,89 & 0,370 \\
\hline
\end{tabular}

Tabelle 4.18: Vergleich der Lebensqualität gemäß Bildungsniveau 


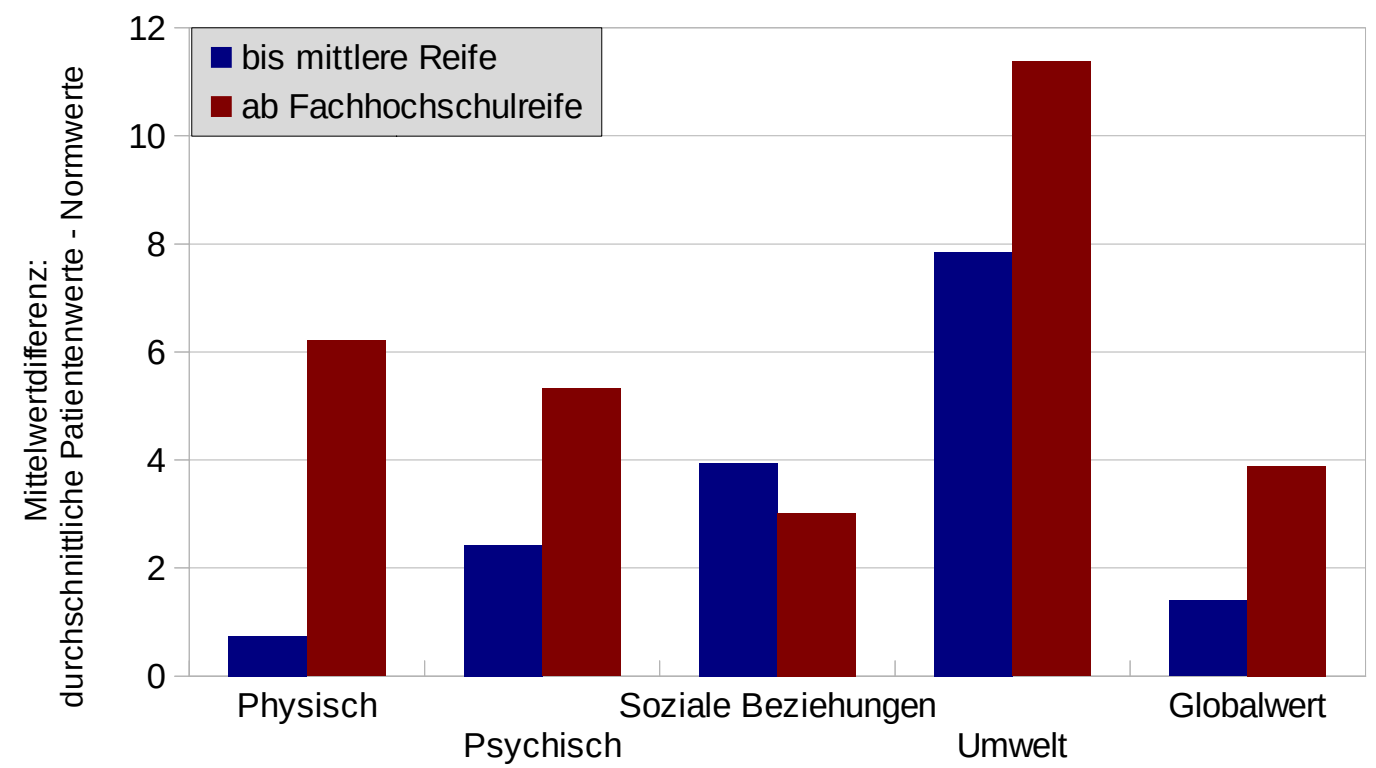

Abbildung 4.13: Vergleich der Lebensqualität gemäß Bildungsniveau

\subsubsection{Auswertung nach dem Familienstand}

In dieser Auswertung sollte festgestellt werden, ob der Familienstand eine Auswirkung auf die Lebensqualität der Patienten dieser Studie hatte.

\subsubsection{Mit Partner lebend}

Mit Partner (verheiratet oder mit Partner lebend) lebten 196 der befragten Patienten. Es ergab sich in allen Domänen eine positive Mittelwertdifferenz und somit eine erhöhte Zufriedenheit mit der Lebensqualität im Vergleich zu den altersbezogenen Normwerten. In den Domänen „Psychisch“, „Soziale Beziehung“ und „Umwelt“ hatte die höhere Zufriedenheit eine statistische Signifikanz und fiel in der Domäne „Umwelt“ am höchsten aus (Mittelwertdifferenz 9,78). Eine Übersicht findet sich in Tabelle 4.19 und Abbildung 4.14.

\begin{tabular}{|l|c|c|c|c|}
\hline & $\begin{array}{c}\text { altersstandardisierte } \\
\text { Normwerte }\end{array}$ & Patientenmittelwerte & Mittelwertdifferenz & $\mathrm{p}$ \\
\hline Physisch & 66,79 & 69,19 & 2,40 & 0,086 \\
\hline Psychisch & 69,18 & 72,98 & 3,80 & $<\mathbf{0 , 0 0 1}$ \\
\hline Soziale Beziehung & 66,76 & 71,13 & 4,37 & $<\mathbf{0 , 0 0 1}$ \\
\hline Umwelt & 68,59 & 78,37 & 9,78 & $<\mathbf{0 , 0 0 1}$ \\
\hline Globalwert & 61,43 & 63,53 & 2,10 & 0,110 \\
\hline
\end{tabular}

Tabelle 4.19: Vergleich der Lebensqualität von mit Partner lebenden Patienten mit den altersstandardisierten Normwerten 


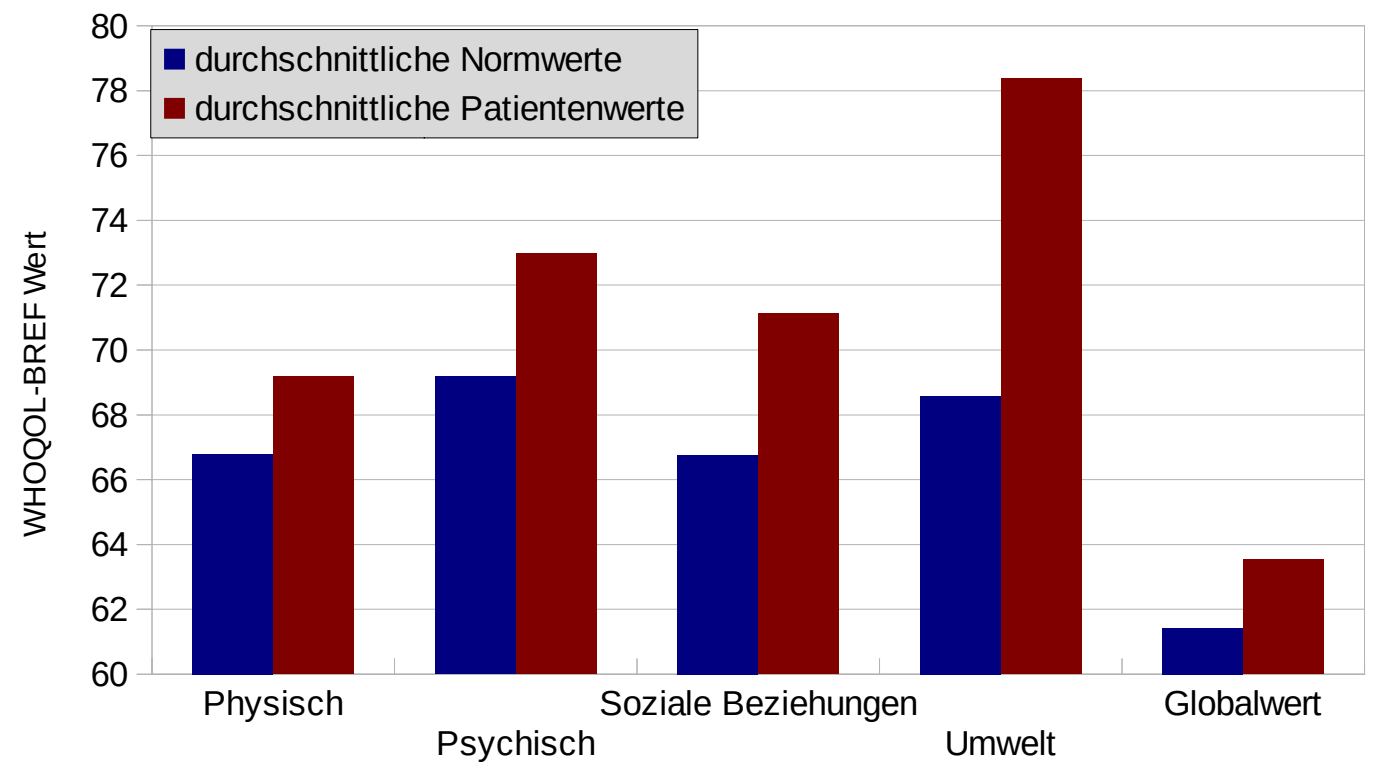

Abbildung 4.14: Vergleich der Lebensqualität von mit Partner lebenden Patienten mit den altersstandardisierten Normwerten

\subsubsection{Ohne Partner lebend}

Hierbei handelte es sich um 64 Patienten, die entweder verwitwet oder geschieden waren, allein oder getrennt lebten. Es zeigten sich in allen Domänen positive Mittelwertdifferenzen, jedoch waren die Unterschiede lediglich in der Domäne „Umwelt“ signifikant. Eine Aufführung der Daten findet sich in Tabelle 4.20 und Abbildung 4.15.

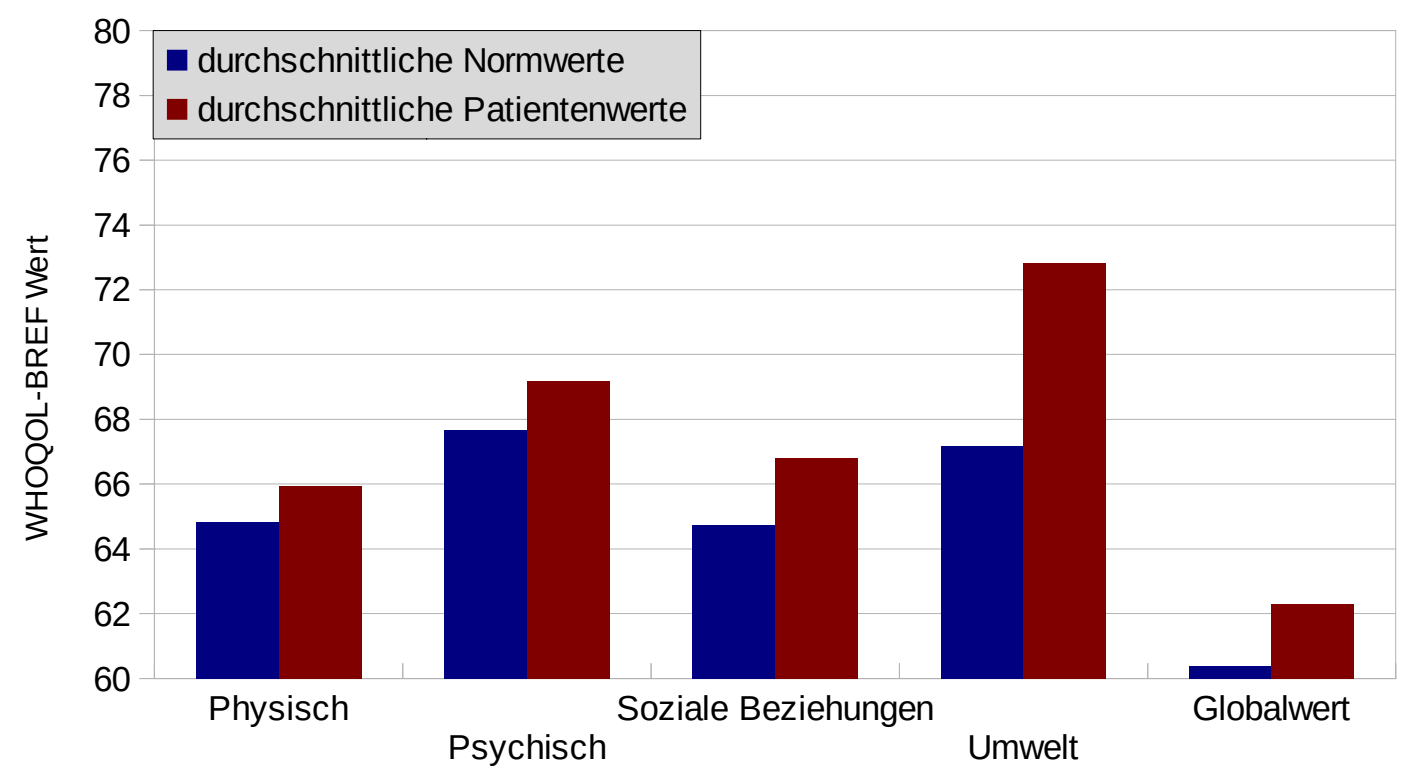

Abbildung 4.15: Vergleich der Lebensqualität von ohne Partner lebenden Patienten mit den altersstandardisierten Normwerten 


\begin{tabular}{|l|c|c|c|c|}
\hline & $\begin{array}{c}\text { altersstandardisierte } \\
\text { Normwerte }\end{array}$ & Patientenmittelwerte & Mittelwertdifferenz & $\mathrm{p}$ \\
\hline Physisch & 64,82 & 65,95 & 1,13 & 0,639 \\
\hline Psychisch & 67,68 & 69,17 & 1,49 & 0,486 \\
\hline Soziale Beziehung & 64,73 & 66,80 & 2,07 & 0,437 \\
\hline Umwelt & 67,17 & 72,81 & 5,64 & $\mathbf{0 , 0 1 0}$ \\
\hline Globalwert & 60,40 & 62,30 & 1,90 & 0,457 \\
\hline
\end{tabular}

Tabelle 4.20: Vergleich der Lebensqualität von ohne Partner lebenden Patienten mit den altersstandardisierten Normwerten

\subsubsection{Vergleich der Unterschiede}

Im Vergleich beider Gruppen zeigte sich, dass die Patienten, die mit Partner lebten, in allen Domänen eine höhere Zufriedenheit mit ihrer Lebensqualität empfanden. Dieser Unterschied hatte seine größte Ausprägung in der Domäne „Umwelt“ und zeigten sich hier auch signifikant. Mit dem allgemeinen Gesundheitszustand („Globalwerte“) zeigten sich beide Gruppen ähnlich zufrieden (Tabelle 4.21 und Abbildung 4.16).

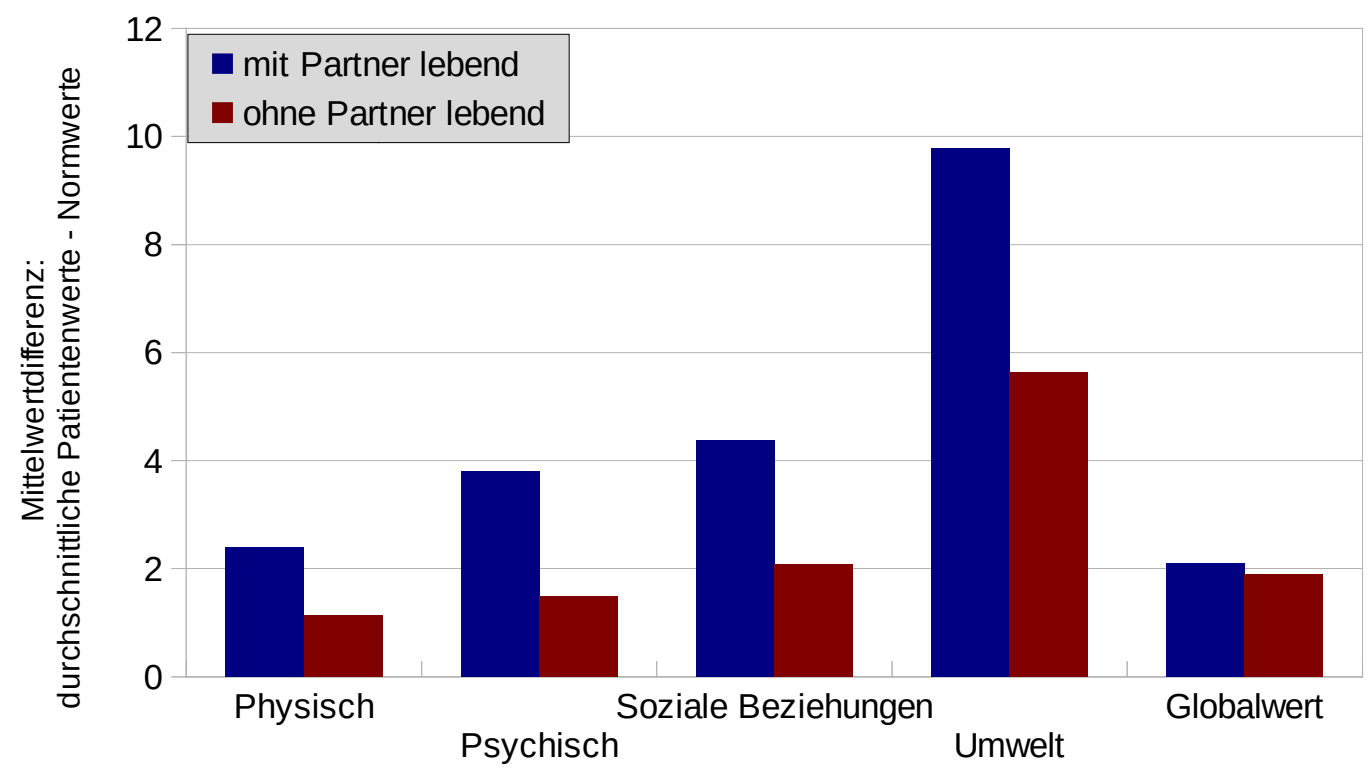

Abbildung 4.16: Vergleich der Lebensqualität gemäß Familienstand 


\begin{tabular}{|l|c|c|c|}
\hline & $\begin{array}{c}\text { Mittelwertdifferenz } \\
\text { zusammen lebend }\end{array}$ & $\begin{array}{c}\text { Mittelwertdifferenz } \\
\text { alleine lebend }\end{array}$ & $\mathrm{p}$ \\
\hline Physisch & 2,40 & 1,13 & 0,650 \\
\hline Psychisch & 3,80 & 1,49 & 0,299 \\
\hline Soziale Beziehung & 4,37 & 2,07 & 0,357 \\
\hline Umwelt & 9,78 & 5,64 & $\mathbf{0 , 0 3 2}$ \\
\hline Globalwert & 2,10 & 1,90 & 0,943 \\
\hline
\end{tabular}

Tabelle 4.21: Vergleich der Lebensqualität gemäß Familienstand 


\subsection{Ergebnisse des Homburger Gesundheitsbogens}

Bei diesem Fragebogen wurden die Patienten gebeten, 11 Fragen mittels fünfstufiger Likert-Skala, und 4 Fragen mit jeweils zwei Antwortmöglichkeiten zu beantworten. Es sollte ergründet werden, inwiefern sich nach Bekanntwerden der Hauttumorerkrankung das Gesundheitsbewusstsein verändert hatte. Die Fragen konnten daraufhin verschiedenen Rubriken zugeführt werden (Sonnenexposition, Ernährung, Freizeitverhalten usw.). Bei den 11 Fragen, die mittels der fünfstufigen Likert-Skala zu beantworten waren, zeigte eine Antwort mit 1-3 (,trifft voll zu“, „trifft weitgehend zu“ oder „trifft ziemlich zu“), dass eine Veränderung im Vergleich zum ursprünglichen Verhalten stattgefunden hatte, wohingegen eine Antwort mit 4 und 5 (,trifft kaum“ zu und „trifft gar nicht zu“) indizierte, dass das alte Verhalten beibehalten wurde. In den einzelnen Rubriken wurde zudem ein Vergleich der verschiedenen Untergruppen vorgenommen. Auf diese Weisen konnten die Ergebnisse geschlechtsspezifisch, je nachdem welche Grunderkrankung (Melanom, nichtmelanozytär bzw. beides), welchen Bildungsgrad, welchen Familienstand oder welches Alter der jeweilige Patient hatte, beleuchtet werden.

\subsubsection{Körperliche Bewegung}

In diesem Teil sollte geprüft werden inwiefern die Patienten sich nach Bekanntwerden ihrer Hauttumorerkrankung mehr körperlich bewegten, und ob sie eine neue Sportart begonnen hatten.

Der Großteil der Patienten (74,8\%; n=195) beantwortete die Frage, ob sie sich nach Bekanntwerden ihrer Hauttumorerkrankung vermehrt körperlich bewegten, mit „trifft gar nicht zu“ oder „trifft kaum zu“. Von den 25,2\% $(n=66)$ die dazu bereit waren ihr Verhalten zu ändern, antworteten 5,7\% ( $n=15)$

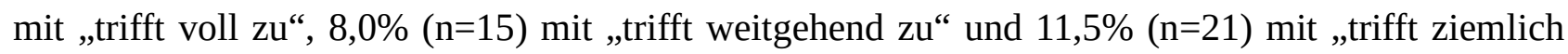
zu“. Eine graphische Darstellung findet sich in Abbildung 4.17. 2 Patienten beantworteten die Frage nicht.

Auf die Frage, ob nach Diagnosestellung eine neue Sportart begonnen wurde, antworteten 11,0\% ( $\mathrm{n}=29$ ) der Patienten mit „trifft voll zu“, „trifft weitgehend zu“ oder „trifft ziemlich zu“. 89,0\% ( $n=232)$ hatten keine neue Sportart angefangen, antworteten also mit „trifft kaum“ bzw. „trifft gar nicht zu“ (Abbildung 4.18). 2 Patienten hatten diese Frage nicht beantwortet. 


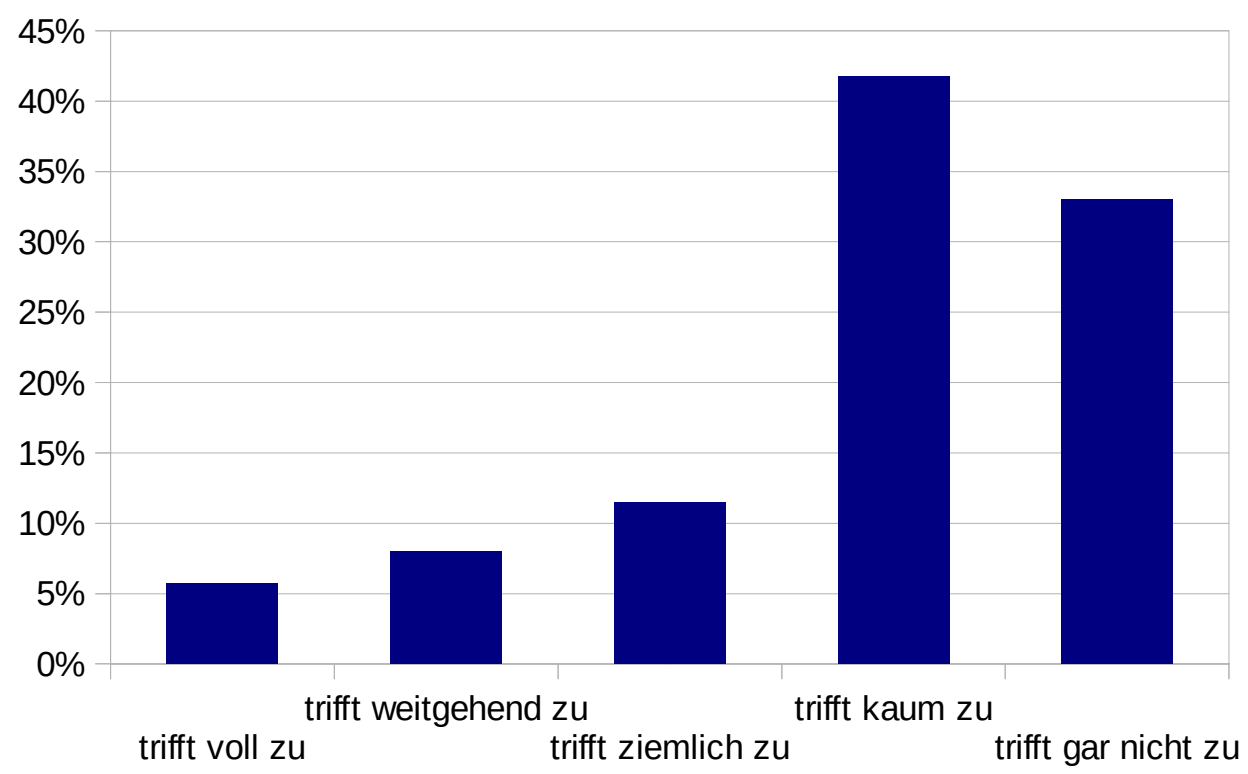

Abbildung 4.17: ...bewege ich mich körperlich mehr als vorher

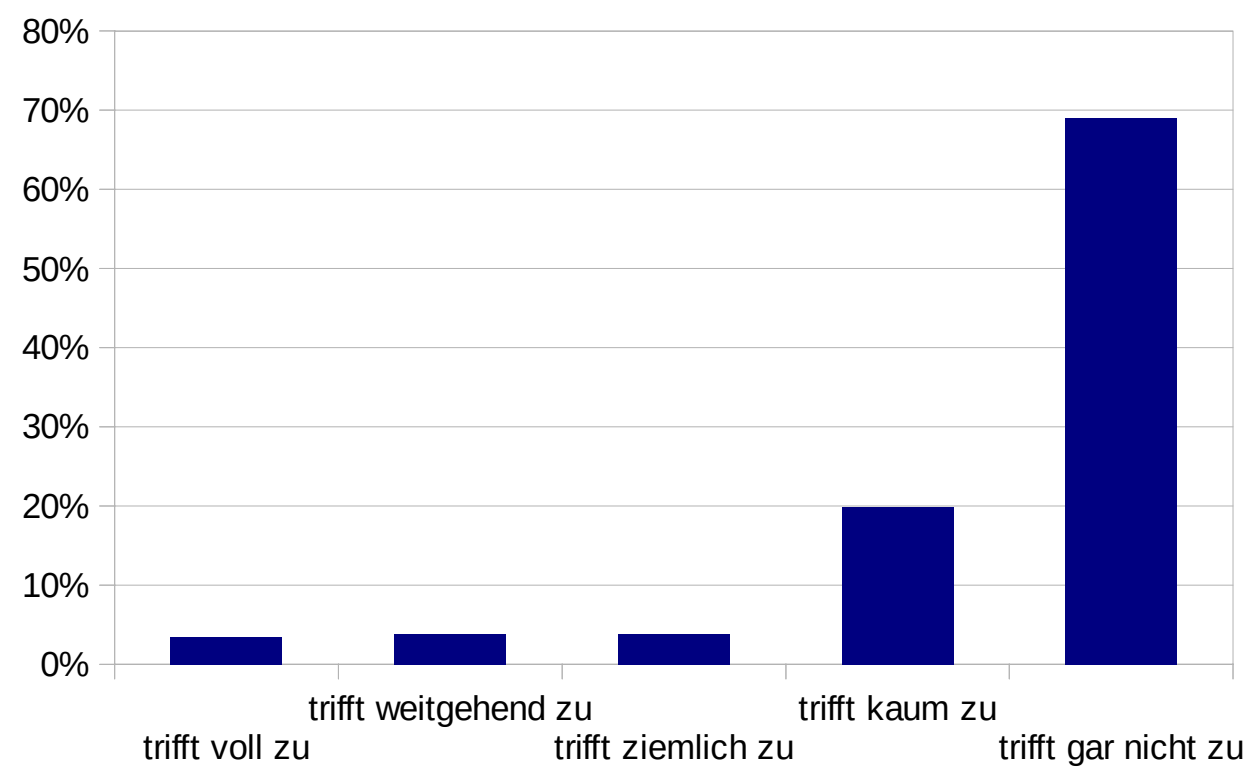

Abbildung 4.18: ... habe ich eine neue Sportart angefangen, die ich nun regelmäßig verfolge

\subsubsection{Geschlechtsspezifische Auswertung}

Zwischen Männern und Frauen zeigten sich bezüglich der körperlichen Bewegung lediglich geringfügige Unterschiede. Bei den Männern waren es 25,1\% $(n=41)$ die sich mehr bewegten, bei den Frauen 25,5\% ( $n=25)$. Der Unterschied war nicht signifikant $(\mathrm{p}=0,366)$ Eine detaillierte Aufstellung der Ergebnisse ist Tabelle 4.22 zu entnehmen. 


\begin{tabular}{|l|l|l|}
\hline & m & W \\
\hline Trifft voll zu & $5,5 \%$ & $6,1 \%$ \\
\hline Trifft weitgehend zu & $6,7 \%$ & $10,2 \%$ \\
\hline Trifft ziemlich zu & $12,9 \%$ & $9,2 \%$ \\
\hline Trifft kaum zu & $39,3 \%$ & $45,9 \%$ \\
\hline Trifft gar nicht zu & $35,6 \%$ & $28,6 \%$ \\
\hline P-Wert & & \\
\hline
\end{tabular}

Tabelle 4.22: ...bewege ich mich körperlich mehr als vorher (geschlechtsspezifische Auswertung)

Auch bei der Frage, ob eine neue Sportart begonnen wurde, zeigten sich keine signifikanten geschlechtsspezifischen Unterschiede ( $\mathrm{p}=0,661)$. Bei den Männern waren es 88,9\% ( $\mathrm{n}=145)$, bei den Frauen 88,7\% (n=87), die nicht mehr Sport ausübten als vor Bekanntwerden ihrer Tumorerkrankung (Tabelle 4.23).

\begin{tabular}{|l|l|l|}
\hline & m & W \\
\hline Trifft voll zu & $1,8 \%$ & $6,1 \%$ \\
\hline Trifft weitgehend zu & $5,5 \%$ & $1,0 \%$ \\
\hline Trifft ziemlich zu & $3,7 \%$ & $4,1 \%$ \\
\hline Trifft kaum zu & $19,0 \%$ & $21,4 \%$ \\
\hline Trifft gar nicht zu & $69,9 \%$ & $67,3 \%$ \\
\hline P-Wert & & \\
\hline
\end{tabular}

Tabelle 4.23: ... habe ich eine neue Sportart begonnen, die ich regelmäßig verfolge (geschlechtsspezifische Auswertung)

\subsubsection{Auswertung nach der Grunddiagnose}

Von den Melanompatienten bewegten sich 21,5\% ( $n=17)$ mehr, bei den Patienten mit nichtmelanozytären Tumoren waren es 23,6\% ( $n=33)$, und bei den Patienten, die unter beiden Tumorentitäten litten, bewegten sich 38,1\% ( $\mathrm{n=16)}$ mehr als vor Diagnosestellung. Zwar zeigten insbesondere die Patienten, die unter beiden Tumorentitäten litten, eine höhere Bereitschaft, sich mehr zu bewegen, als dies bei den anderen Gruppen der Fall war, jedoch war der Unterschied nicht statistisch signifikant $(\mathrm{p}=0,095)$ Eine genaue Aufstellung der Ergebnisse ist Tabelle $4.24 \mathrm{zu}$ entnehmen. 


\begin{tabular}{|l|l|l|l|}
\hline & Melanom & $\begin{array}{l}\text { Nicht-melanozytäre } \\
\text { Tumoren }\end{array}$ & $\begin{array}{l}\text { Melanom und } \\
\text { Nicht-melanozytäre } \\
\text { Tumoren }\end{array}$ \\
\hline Trifft voll zu & $7,6 \%$ & $3,6 \%$ & $9,5 \%$ \\
\hline Trifft weitgehend zu & $6,3 \%$ & $6,4 \%$ & $16,7 \%$ \\
\hline Trifft ziemlich zu & $7,6 \%$ & $13,6 \%$ & $11,9 \%$ \\
\hline Trifft kaum zu & $50,6 \%$ & $38,6 \%$ & $35,7 \%$ \\
\hline Trifft gar nicht zu & $27,8 \%$ & $37,9 \%$ & $26,2 \%$ \\
\hline P-Wert & & & 0,095 \\
\hline
\end{tabular}

Tabelle 4.24: ... bewege ich mich körperlich mehr als vorher (Auswertung nach Grunddiagnose)

Auf die Frage, ob eine neue Sportart begonnen wurde, antworteten 16,4\% ( $\mathrm{n}=13)$ der Melanompatienten mit „trifft voll zu“, „trifft weitgehend zu“ oder „trifft ziemlich zu“, bei Patienten mit beiden Tumoren waren es $14,4 \%(n=6)$, und bei den Patienten mit nicht melanozytären Tumoren waren es mit 7,1\% $(n=10)$ am wenigsten. Die Ergebnisse zeigten sich mit $\mathrm{p}=0,055$ nicht statistisch signifikant (Tabelle 4.25).

\begin{tabular}{|l|l|l|l|}
\hline & Melanom & $\begin{array}{l}\text { Nicht-melanozytäre } \\
\text { Tumoren }\end{array}$ & $\begin{array}{l}\text { Melanom und } \\
\text { Nicht-melanozytäre } \\
\text { Tumoren }\end{array}$ \\
\hline Trifft voll zu & $3,8 \%$ & $2,9 \%$ & $4,8 \%$ \\
\hline Trifft weitgehend zu & $6,3 \%$ & $2,1 \%$ & $4,8 \%$ \\
\hline Trifft ziemlich zu & $6,3 \%$ & $2,1 \%$ & $4,8 \%$ \\
\hline Trifft kaum zu & $21,5 \%$ & $17,9 \%$ & $23,8 \%$ \\
\hline Trifft gar nicht zu & $62,0 \%$ & $75,0 \%$ & $61,9 \%$ \\
\hline P-Wert & & & 0,055 \\
\hline
\end{tabular}

Tabelle 4.25: ...habe ich eine neue Sportart begonnen, die ich regelmäßig verfolge (Auswertung nach Grunddiagnose)

\subsubsection{Auswertung nach dem Schulabschluss}

28,0\% ( $\mathrm{n}=55)$ der Patienten mit niedrigerem Qualifikationsniveau (Schulabschluss bis einschließlich mittlerer Reife) bewegten sich mehr als noch vor Diagnosestellung. Bei den Patienten mit höherem Qualifikationsniveau (Schulabschluss ab Fachhochschulreife) waren es mit 18,0\% $(n=11)$ weniger. Dieser Unterschied zeigte sich nicht signifikant $(\mathrm{p}=0,051)$. Die einzelnen Werte finden sich in Tabelle 4.26. 


\begin{tabular}{|l|l|l|}
\hline & bis einschließlich mittlere Reife & ab Fachhochschulreife \\
\hline Trifft voll zu & $6,1 \%$ & $4,9 \%$ \\
\hline Trifft weitgehend zu & $10,7 \%$ & $0,0 \%$ \\
\hline Trifft ziemlich zu & $11,2 \%$ & $13,1 \%$ \\
\hline Trifft kaum zu & $41,6 \%$ & $41,0 \%$ \\
\hline Trifft gar nicht zu & $30,5 \%$ & $41,0 \%$ \\
\hline P-Wert & & \\
\hline
\end{tabular}

Tabelle 4.26: ...bewege ich mich körperlich mehr als vorher (Auswertung nach Schulabschluss)

Bei der Frage, ob nach Diagnosestellung eine neue Sportart begonnen wurde, antworteten von den Befragten mit niedrigerer Qualifikation 10,7\% ( $=21)$ mit „trifft voll zu“, „trifft weitgehend zu“ oder „trifft ziemlich zu“. Bei Patienten mit höherer Qualifikation waren es mit 13,1\% (n=8) etwas mehr. Der Unterschied war nicht signifikant ( $\mathrm{p}=0,94)$. In beiden Gruppen beantworteten über 68\% die Frage mit „trifft gar nicht zu“ (Tabelle 4.27).

\begin{tabular}{|l|l|l|}
\hline & bis einschließlich mittlere Reife & ab Fachhochschulreife \\
\hline Trifft voll zu & $3,0 \%$ & $4,9 \%$ \\
\hline Trifft weitgehend zu & $4,1 \%$ & $3,3 \%$ \\
\hline Trifft ziemlich zu & $3,6 \%$ & $4,9 \%$ \\
\hline Trifft kaum zu & $20,8 \%$ & $18,0 \%$ \\
\hline Trifft gar nicht zu & $68,5 \%$ & $68,9 \%$ \\
\hline P-Wert & & \\
\hline
\end{tabular}

Tabelle 4.27: ...habe ich eine neue Sportart begonnen, die ich regelmäßig verfolge (Auswertung nach Schulabschluss)

\subsubsection{Auswertung nach dem Familienstand}

25,1\% ( $\mathrm{n}=48)$ der Befragten, die mit Partner lebten, bewegten sich mehr als das noch vor Diagnosestellung der Fall war. Bei Patienten, die ohne Partner lebten, waren es mit 26,6\% (n=17) geringfügig mehr (Tabelle 4.28). Der Unterschied zwischen beiden Gruppen war nicht signifikant $(p=0,229)$.

Bei den Patienten die mit Partner lebten begannen 11,3\% $(n=22)$ eine neue Sportart. Bei Patienten ohne Partner waren es mit 11,0\% ( $n=7)$ ebenso viele (Tabelle 4.29). Dementsprechend war der Unterschied nicht signifikant $(\mathrm{p}=0,798)$. 


\begin{tabular}{|c|c|c|}
\hline & mit Partner & ohne Partner \\
\hline Trifft voll zu & $5,6 \%$ & $6,3 \%$ \\
\hline Trifft weitgehend zu & $7,2 \%$ & $10,9 \%$ \\
\hline Trifft ziemlich zu & $12,3 \%$ & $9,4 \%$ \\
\hline Trifft kaum zu & $39,5 \%$ & $48,4 \%$ \\
\hline Trifft gar nicht zu & $35,4 \%$ & $25,0 \%$ \\
\hline P-Wert & & 0,229 \\
\hline
\end{tabular}

Tabelle 4.28: ...bewege ich mich körperlich mehr als vorher (Auswertung nach Familienstand)

\begin{tabular}{|l|l|l|}
\hline & mit Partner & ohne Partner \\
\hline Trifft voll zu & $3,1 \%$ & $4,7 \%$ \\
\hline Trifft weitgehend zu & $4,6 \%$ & $1,6 \%$ \\
\hline Trifft ziemlich zu & $3,6 \%$ & $4,7 \%$ \\
\hline Trifft kaum zu & $19,5 \%$ & $21,9 \%$ \\
\hline Trifft gar nicht zu & $69,2 \%$ & $67,2 \%$ \\
\hline P-Wert & & \\
\hline
\end{tabular}

Tabelle 4.29: ...habe ich eine neue Sportart begonnen, die ich regelmäßig verfolge (Auswertung nach Familienstand)

\subsubsection{Auswertung nach dem Alter}

Mit 28,0\% ( $n=24)$ waren es eher die jüngeren Patienten (bis 65 Jahre), die sich mehr bewegten als noch vor der Diagnosestellung. Bei den älteren Patienten (ab 66 Jahre) waren es 24,0\% (n=42) die die Frage mit „trifft voll zu“, „trifft weitgehend zu“ oder „trifft ziemlich zu“ beantworteten (Tabelle 4.30). Der Unterschied zwischen beiden Gruppen war nicht signifikant ( $p=0,282)$.

Ein statistisch signifikanter Unterschied $(p=0,001)$ ergab sich bei der Frage, ob die Patienten nach Bekanntwerden ihrer Hauttumorerkrankung eine neue Sportart begonnen hatten.Zwar zeigte sich in beiden Gruppen, dass kaum Patienten ihr Verhalten geändert hatten, allerdings waren bei den jüngeren Patienten immerhin 18,6\% ( $\mathrm{n}=16)$, bei den älteren Patienten hingegen nur 7,4\% $(\mathrm{n}=13)$ bereit, eine neue Sportart zu beginnen (Tabelle 4.31 und Abbildung 4.20). 


\begin{tabular}{|l|l|l|}
\hline & Unter 65 & Über 66 \\
\hline Trifft voll zu & $7,0 \%$ & $5,1 \%$ \\
\hline Trifft weitgehend zu & $10,5 \%$ & $6,9 \%$ \\
\hline Trifft ziemlich zu & $10,5 \%$ & $12,0 \%$ \\
\hline Trifft kaum zu & $43,0 \%$ & $41,1 \%$ \\
\hline Trifft gar nicht zu & $29,1 \%$ & $34,9 \%$ \\
\hline P-Wert & & \\
\hline
\end{tabular}

Tabelle 4.30: ...bewege ich mich körperlich mehr als vorher (Auswertung nach Alter)

\begin{tabular}{|l|l|l|}
\hline & Unter 65 & Über 66 \\
\hline Trifft voll zu & $8,1 \%$ & $1,1 \%$ \\
\hline Trifft weitgehend zu & $5,8 \%$ & $2,9 \%$ \\
\hline Trifft ziemlich zu & $4,7 \%$ & $3,4 \%$ \\
\hline Trifft kaum zu & $24,4 \%$ & $17,7 \%$ \\
\hline Trifft gar nicht zu & $57,0 \%$ & $74,9 \%$ \\
\hline P-Wert & & \\
\hline
\end{tabular}

Tabelle 4.31: ...habe ich eine neue Sportart begonnen, die ich regelmäßig verfolge (Auswertung nach Alter)

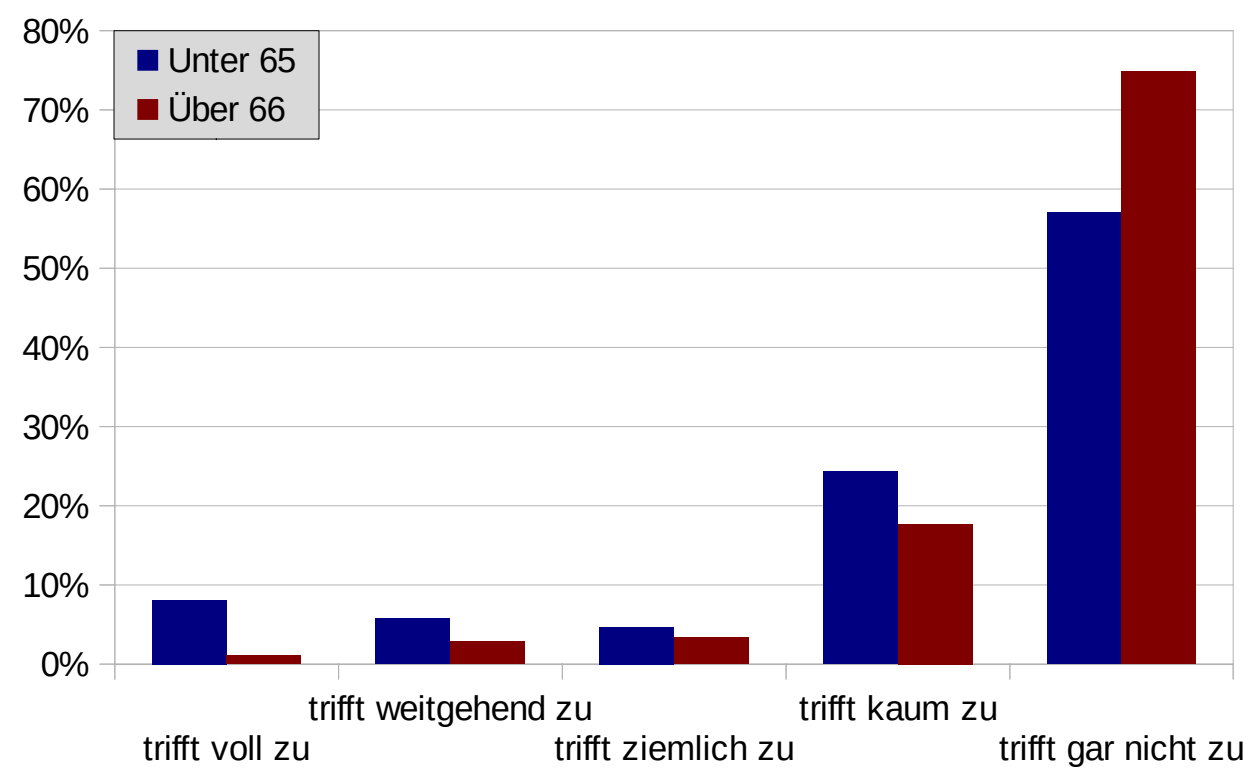

Abbildung 4.19: ...habe ich eine neue Sportart begonnen, die ich regelmäßig verfolge (Auswertung nach Alter)

\subsubsection{Sonnenexposition}

Auf die Frage, ob darauf geachtet wurde, sich weniger in der Sonne aufzuhalten, hatten 88,5\% 
( $n=232)$ ihr Verhalten nach Bekanntwerden der Tumorerkrankung verändert. Hiervon antworteten 48,5\% ( $n=127)$ der Patienten mit „trifft voll zu“. Nur 11,5\% $(n=30)$ der Befragten änderten ihr Verhalten kaum oder gar nicht (Abbildung 4.20). Die Frage blieb durch einen Patienten unbeantwortet.

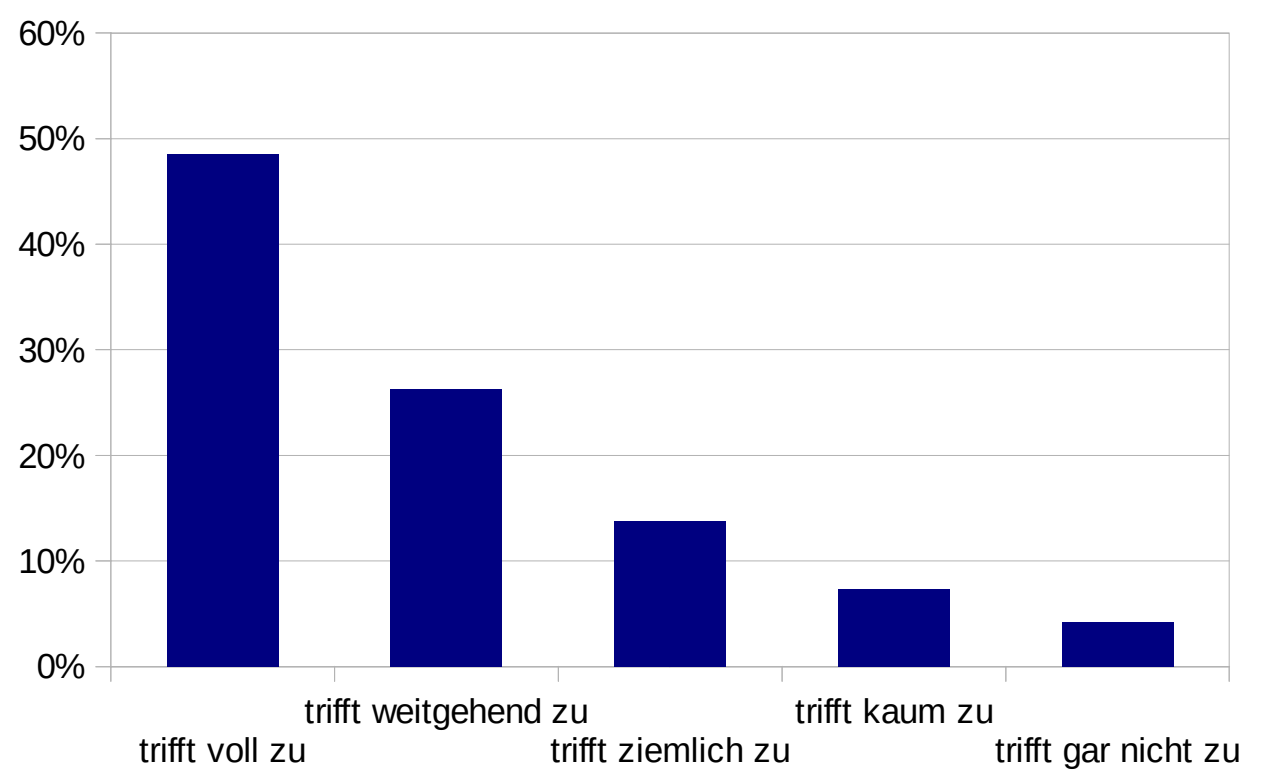

Abbildung 4.20: ... achte ich mehr darauf, mich nicht lange in der Sonne aufzuhalten

\subsubsection{Geschlechtsspezifische Auswertung}

54\% ( $n=54)$ der Frauen gegenüber 45,1\% (n=73) bei den Männern antworteten auf die Frage, ob sie die Aufenthaltsdauer in der Sonne nach Bekanntwerden der Hauttumorerkrankung reduziert hätten, mit „trifft voll zu“. Weitere 37\% ( $\mathrm{n}=37)$ der Frauen antworteten mit „trifft weitgehend“ bzw. „trifft ziemlich zu“. Bei den Männern waren es weitere 41,9\% (n=68). Dieser Unterschied war allerdings nicht signifikant ( $p=0,096)$ (Tabelle 4.32).

\begin{tabular}{|l|l|l|}
\hline & m & W \\
\hline Trifft voll zu & $45,1 \%$ & $54,0 \%$ \\
\hline Trifft weitgehend zu & $25,9 \%$ & $27,0 \%$ \\
\hline Trifft ziemlich zu & $16,0 \%$ & $10,0 \%$ \\
\hline Trifft kaum zu & $9,3 \%$ & $4,0 \%$ \\
\hline Trifft gar nicht zu & $3,7 \%$ & $5,0 \%$ \\
\hline P-Wert & & \\
\hline
\end{tabular}

Tabelle 4.32: ... achte ich mehr darauf, mich nicht lange in der Sonne aufzuhalten (geschlechtsspezifische Auswertung) 


\subsubsection{Auswertung nach der Grunddiagnose}

80,8\% ( $n=63)$ der Melanompatienten achteten darauf, sich nicht mehr soviel in der Sonne aufzuhalten wie noch vor ihrer Hautkrebserkrankung. Bei den Patienten mit nicht-melanozytären Tumoren waren es mit 90,8\% ( $n=128)$ mehr, und bei den Patienten, die unter beiden Tumorentitäten litten, waren es mit 95,3\% (n=41) am meisten (Tabelle 4.33). Der Unterschied erwies sich jedoch als nicht statistisch signifikant $(\mathrm{p}=0,533)$.

\begin{tabular}{|l|l|l|l|}
\hline & Melanom & $\begin{array}{l}\text { Nicht-melanozytäre } \\
\text { Tumoren }\end{array}$ & $\begin{array}{l}\text { Melanom und } \\
\text { Nicht-melanozytäre } \\
\text { Tumoren }\end{array}$ \\
\hline Trifft voll zu & $48,7 \%$ & $46,1 \%$ & $55,8 \%$ \\
\hline Trifft weitgehend zu & $23,1 \%$ & $29,8 \%$ & $20,9 \%$ \\
\hline Trifft ziemlich zu & $9,0 \%$ & $14,9 \%$ & $18,6 \%$ \\
\hline Trifft kaum zu & $12,8 \%$ & $5,0 \%$ & $4,7 \%$ \\
\hline Trifft gar nicht zu & $6,4 \%$ & $4,3 \%$ & $0,0 \%$ \\
\hline P-Wert & & & \\
\hline
\end{tabular}

Tabelle 4.33: ...achte ich mehr darauf, mich nicht lange in der Sonne aufzuhalten (Auswertung nach der Grunddiagnose)

\subsubsection{Auswertung nach dem Schulabschluss}

Sowohl die Patienten mit niedrigerer Qualifikation (90,8\%; $n=179)$, als auch jene mit höherer Qualifikation (82,0\%; $\mathrm{n}=50$ ) verminderten die Sonnenexposition nach Bekanntwerden ihrer Tumorerkrankung vollständig oder zum Teil (Tabelle 4.34). Einen signifikanten Unterschied zwischen den Gruppen gab es nicht $(\mathrm{p}=0,074)$.

\begin{tabular}{|l|l|l|}
\hline & bis einschließlich mittlere Reife & ab Fachhochschulreife \\
\hline Trifft voll zu & $49,7 \%$ & $44,3 \%$ \\
\hline Trifft weitgehend zu & $29,4 \%$ & $16,4 \%$ \\
\hline Trifft ziemlich zu & $11,7 \%$ & $21,3 \%$ \\
\hline Trifft kaum zu & $6,6 \%$ & $9,8 \%$ \\
\hline Trifft gar nicht zu & $2,5 \%$ & $8,2 \%$ \\
\hline P-Wert & & \\
\hline
\end{tabular}

Tabelle 4.34: ...achte ich mehr darauf, mich nicht lange in der Sonne aufzuhalten (Auswertung nach Schulabschluss) 


\subsubsection{Auswertung nach dem Familienstand}

Patienten, die mit Partner lebten, verminderten die Zeit der Sonnenexposition in 88,7\% (n=173) der

Fälle. Bei Patienten ohne Partner beantworteten 89,1\% ( $n=57)$ die Frage mit „trifft voll zu“, „trifft weitgehend“ bzw. „trifft ziemlich zu“ (Tabelle 4.35). Einen signifikanten Unterschied zwischen den Gruppen gab es nicht ( $\mathrm{p}=0,399)$.

\begin{tabular}{|l|l|l|}
\hline & mit Partner & ohne Partner \\
\hline Trifft voll zu & $50,3 \%$ & $43,8 \%$ \\
\hline Trifft weitgehend zu & $25,6 \%$ & $28,1 \%$ \\
\hline Trifft ziemlich zu & $12,8 \%$ & $17,2 \%$ \\
\hline Trifft kaum zu & $7,7 \%$ & $6,3 \%$ \\
\hline Trifft gar nicht zu & $3,6 \%$ & $4,7 \%$ \\
\hline P-Wert & & \\
\hline
\end{tabular}

Tabelle 4.35: ...achte ich mehr darauf, mich nicht lange in der Sonne aufzuhalten (Auswertung nach Familienstand)

\subsubsection{Auswertung nach dem Alter}

90,3\% ( $n=159)$ der älteren Patienten reduzierten ihre Sonnenexposition nach Bekanntwerden der Hauttumorerkrankung. Bei den Patienten unter 65 Jahren waren es mit 84,8\% (n=73) ähnlich viele (Tabelle 4.36). Der Unterschied zwischen beiden Gruppen war nicht statistisch signifikant $(p=0,982)$.

\begin{tabular}{|l|l|l|}
\hline & Unter 65 & Über 66 \\
\hline Trifft voll zu & $48,8 \%$ & $48,3 \%$ \\
\hline Trifft weitgehend zu & $26,7 \%$ & $26,1 \%$ \\
\hline Trifft ziemlich zu & $9,3 \%$ & $15,9 \%$ \\
\hline Trifft kaum zu & $11,6 \%$ & $5,1 \%$ \\
\hline Trifft gar nicht zu & $3,5 \%$ & $4,5 \%$ \\
\hline P-Wert & & \\
\hline
\end{tabular}

Tabelle 4.36: ...achte ich mehr darauf, mich nicht lange in der Sonne aufzuhalten (Auswertung nach Alter) 


\subsubsection{Verwendung von textilen und topischen UV-Schutzmöglichkeiten}

Auf die Frage, ob seit Diagnosestellung Sonnencremes häufiger verwendet wurden als noch davor, antworteten 68,8\% (n=181) mit „trifft voll zu“, „trifft weitgehend“ und „trifft ziemlich zu“. 31,1\% (n=82) hatten ihr Verhalten kaum oder gar nicht verändert (Abbildung 4.21).

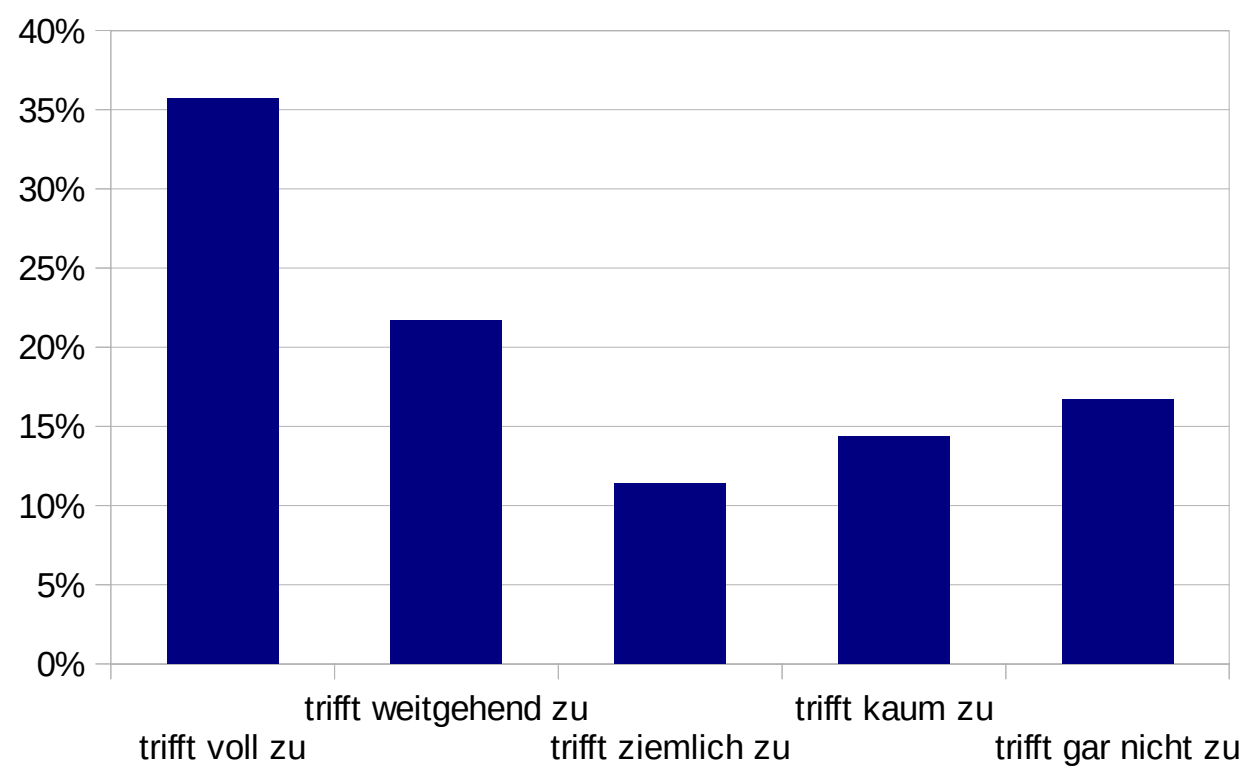

Abbildung 4.21: ...benutze ich häufiger als früher Sonnenschutzcremes mit Lichtschutzfaktor

32,7\% ( $n=86)$ beantworteten die Frage, ob sie nach der Diagnose des Hauttumors regelmäßig einen Sonnenhut trugen, mit „trifft voll zu“. Weitere 25,4\% $(n=80)$ beantworteten die Frage mit „trifft weitgehend“ bzw. „trifft ziemlich zu“. 36,9\% (n=97) trugen nach der Diagnose keine (bzw. selten) eine Kopfbedeckungen (Abbildung 4.22).

Auf die Frage, ob nach Bekanntwerden der Hauttumorerkrankung regelmäßig eine Sonnenbrille getragen wurde, antworteten 23,3\% ( $\mathrm{n}=61)$ mit „trifft voll zu“, 20,2\% (n=53) mit „trifft weitgehend zu“ und 13,7\% (n=36) mit „trifft ziemlich zu“. Fast die Hälfte (42,8\%; n=112) trugen nicht häufiger als früher einen Sonnenbrille. Davon antworteten 21,0\% (n=55) mit „trifft kaum zu“ und 21,8\% (n=57) mit „trifft gar nicht zu“. Ein Patient beantwortete die Frage nicht. 


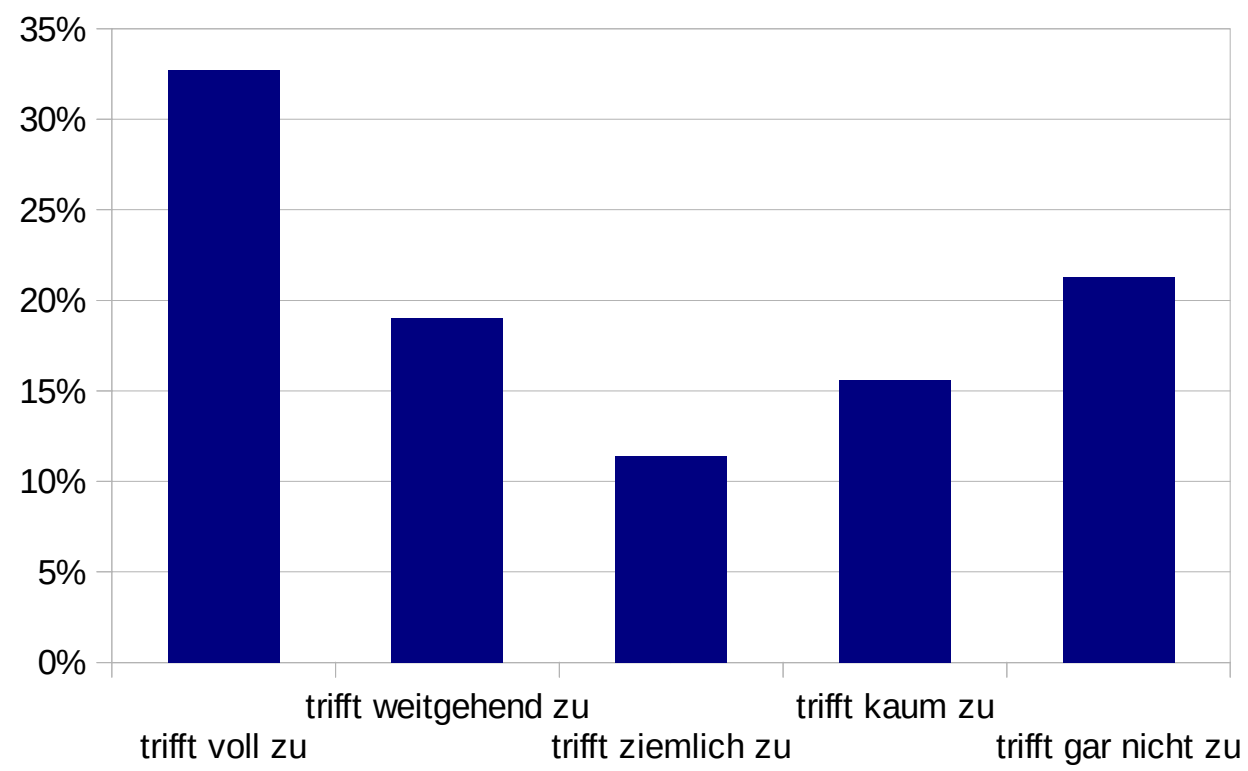

Abbildung 4.22: ...trage ich regelmäßig einen Sonnenhut

\subsubsection{Geschlechtsspezifische Auswertung}

In der geschlechtsspezifischen Aufteilung zeigte sich bezüglich des Aufbringens von Sonnenschutzcremes, dass Frauen nach der Diagnosestellung Sonnencremes häufiger verwendeten als Männer. 81\% (n=81) aller Frauen änderten ihr Verhalten, wovon 45\% (n=45) mit „trifft voll zu“ antworteten. Bei den Männern waren es hingegen nur 61,3\% (n=100) die mit „trifft voll zu“, „trifft

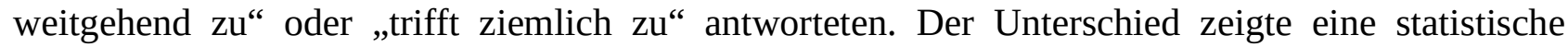
Signifikanz $(\mathrm{p}=0,001)$. Eine graphische Übersicht ist in Tabelle 4.37 und Abbildung 4.23 dargestellt.

Auf die Frage, ob nach Bekanntwerden der Tumorerkrankung ein Sonnenhut getragen wurde zeigte sich eine entgegengesetzte Tendenz. Hier waren es eher die Männer, von denen 74,3\% (n=121) häufiger eine Kopfbedeckung trugen als vor der Diagnose ihrer Hautkrebserkrankung. Bei den Frauen waren es hingegen nur 45\% $(n=45)$. Auch dieses Ergebnis zeigte sich signifikant $(p<0,001)$. Details, sowie eine graphische Darstellung sind Tabelle 4.38 bzw. Abbildung 4.24 zu entnehmen. 


\begin{tabular}{|l|l|l|}
\hline & m & W \\
\hline Trifft voll zu & $30,1 \%$ & $45,0 \%$ \\
\hline Trifft weitgehend zu & $20,2 \%$ & $24,0 \%$ \\
\hline Trifft ziemlich zu & $11,0 \%$ & $12,0 \%$ \\
\hline Trifft kaum zu & $18,4 \%$ & $8,0 \%$ \\
\hline Trifft gar nicht zu & $20,2 \%$ & $11,0 \%$ \\
\hline P-Wert & & \\
\hline
\end{tabular}

Tabelle 4.37: ...benutze ich häufiger als früher Sonnenschutzcremes mit Lichtschutzfaktor (geschlechtsspezifische Auswertung)

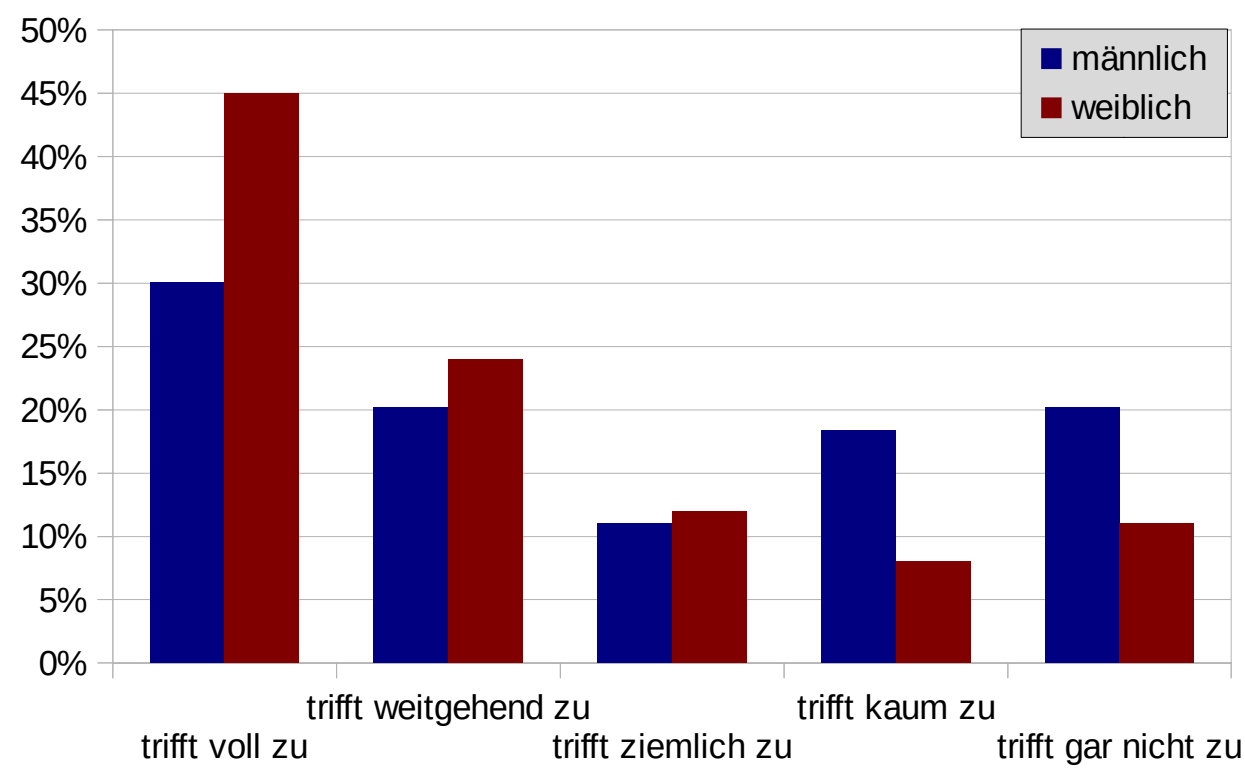

Abbildung 4.23: ...benutze ich häufiger als früher Sonnenschutzcremes mit Lichtschutzfaktor (geschlechtsspezifische Auswertung)

\begin{tabular}{|l|l|l|}
\hline & m & W \\
\hline Trifft voll zu & $39,9 \%$ & $21,0 \%$ \\
\hline Trifft weitgehend zu & $21,5 \%$ & $15,0 \%$ \\
\hline Trifft ziemlich zu & $12,9 \%$ & $9,0 \%$ \\
\hline Trifft kaum zu & $11,0 \%$ & $23,0 \%$ \\
\hline Trifft gar nicht zu & $14,7 \%$ & $32,0 \%$ \\
\hline P-Wert & & \\
\hline
\end{tabular}

Tabelle 4.38: ...trage ich regelmäßig einen Sonnenhut (geschlechtsspezifische Auswertung) 


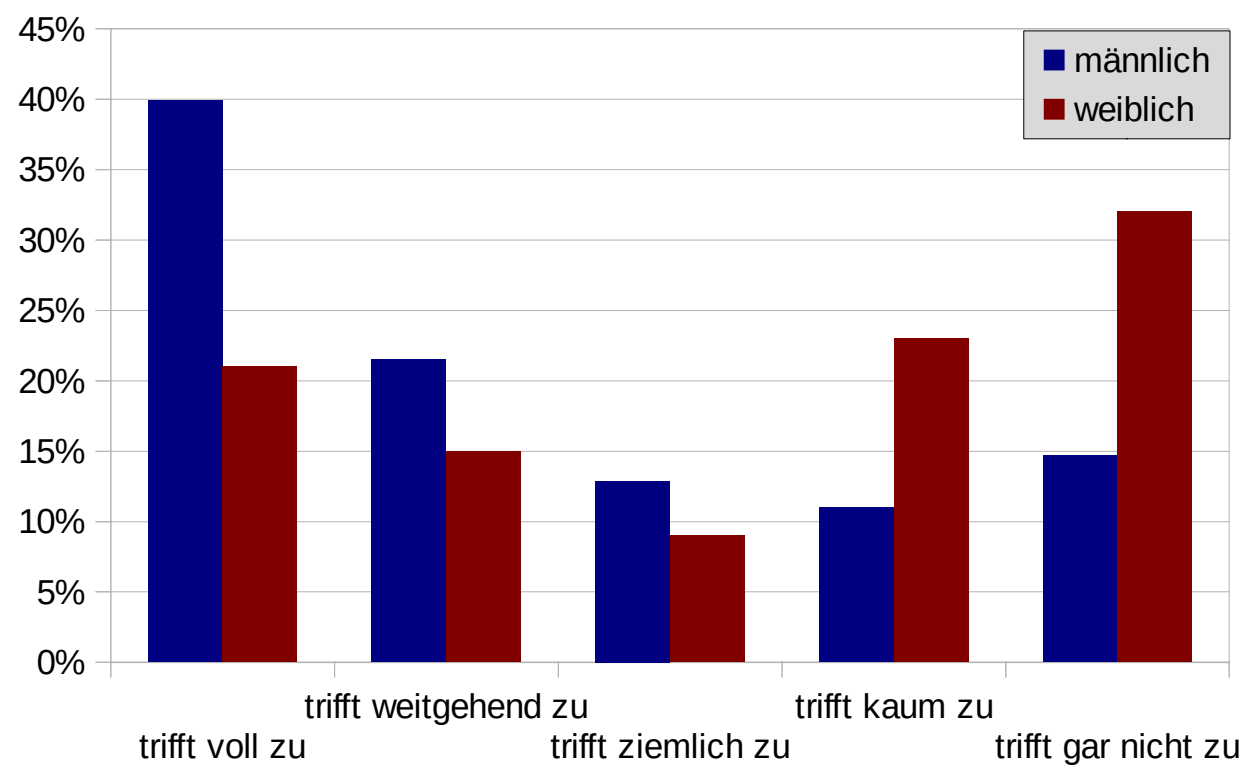

Abbildung 4.24: ...trage ich regelmäßig einen Sonnenhut (geschlechtsspezifische Auswertung)

Die Frage, ob seit Bekanntwerden der Hautkrebserkrankung regelmäßig eine Sonnenbrille getragen wurde, beantworteten 65,0\% ( $\mathrm{n}=65)$ der Frauen mit „trifft voll zu“, „trifft weitgehend“ zu oder „trifft ziemlich zu“. Bei den Männern waren es mit 52,5\% (n=85) weniger (Tabelle 4.39 bzw. Abbildung 4.25). Der Unterschied war statistisch signifikant $(\mathrm{p}=0,008)$.

\begin{tabular}{|l|l|l|}
\hline & m & w \\
\hline Trifft voll zu & $17,3 \%$ & $33,0 \%$ \\
\hline Trifft weitgehend zu & $21,0 \%$ & $19,0 \%$ \\
\hline Trifft ziemlich zu & $14,2 \%$ & $13,0 \%$ \\
\hline Trifft kaum zu & $22,8 \%$ & $18,0 \%$ \\
\hline Trifft gar nicht zu & $24,7 \%$ & $17,0 \%$ \\
\hline P-Wert & & \\
\hline
\end{tabular}

Tabelle 4.39: ...trage ich regelmäßig eine Sonnenbrille (geschlechtsspezifische Auswertung) 


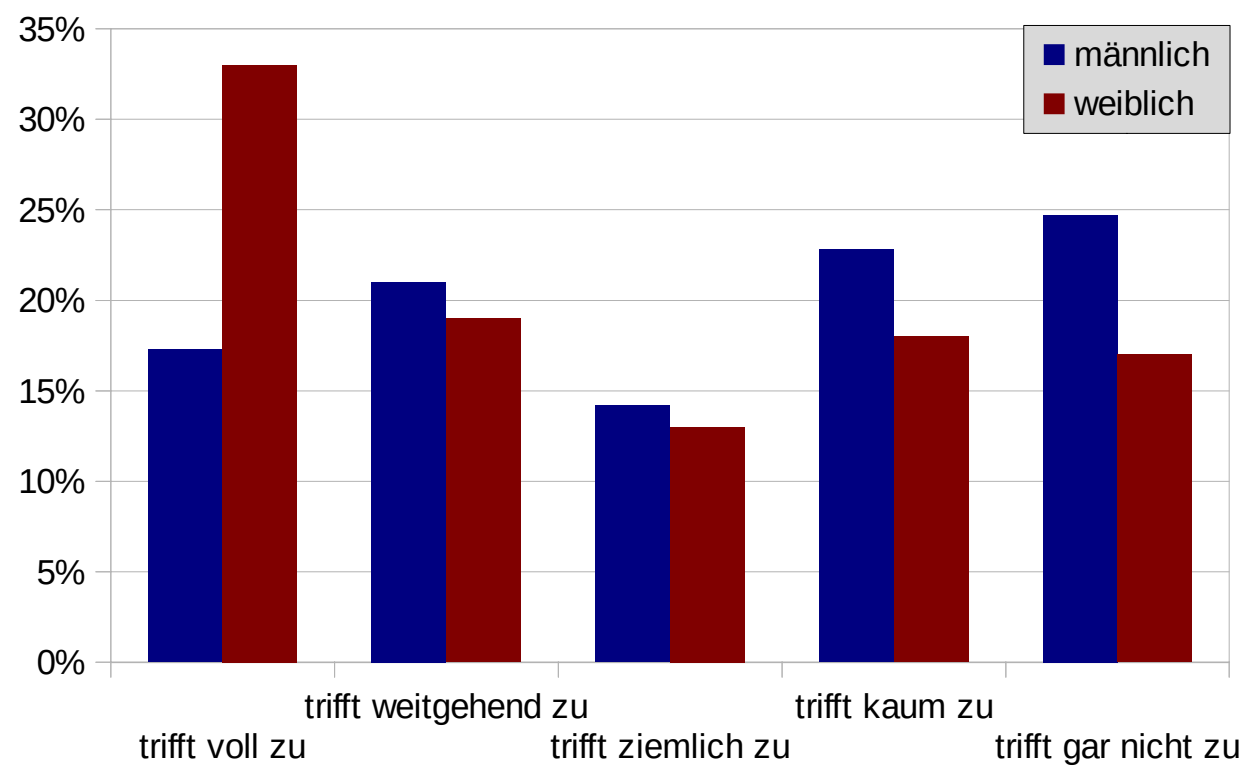

Abbildung 4.25: ...trage ich regelmäßig eine Sonnenbrille (geschlechtsspezifische Auswertung)

\subsubsection{Auswertung nach der Grunddiagnose}

Mit 73,4\% ( $n=58)$ bei den Melanompatienten, und 72,1\% ( $n=31)$ bei Patienten mit Tumoren aus beiden Gruppen, benutzten diese Patienten häufiger Sonnenschutzcremes als Patienten mit nichtmelanozytäten Tumoren (65,3\%; n=92). Details finden sich in Tabelle 4.40. Der Unterschied war nicht signifikant $(\mathrm{p}=0,361)$.

\begin{tabular}{|l|c|c|c|}
\hline & Melanom & $\begin{array}{l}\text { Nicht-melanozytäre } \\
\text { Tumoren }\end{array}$ & $\begin{array}{l}\text { Melanom und } \\
\text { Nicht-melanozytäre } \\
\text { Tumoren }\end{array}$ \\
\hline Trifft voll zu & $36,7 \%$ & $34,8 \%$ & $37,2 \%$ \\
\hline Trifft weitgehend zu & $25,3 \%$ & $17,7 \%$ & $27,9 \%$ \\
\hline Trifft ziemlich zu & $11,4 \%$ & $12,8 \%$ & $7,0 \%$ \\
\hline Trifft kaum zu & $13,9 \%$ & $13,5 \%$ & $18,6 \%$ \\
\hline Trifft gar nicht zu & $12,7 \%$ & $21,3 \%$ & $9,3 \%$ \\
\hline P-Wert & \multicolumn{3}{|c|}{0,361} \\
\hline
\end{tabular}

Tabelle 4.40: ...benutze ich häufiger als früher Sonnenschutzcremes mit Lichtschutzfaktor (Auswertung nach der Grunddiagnose)

Bei der Frage, ob häufiger ein Sonnenhut getragen wurde, waren es vor allem die Patienten mit nicht-melanozytären Tumoren (67,4\%; $n=95)$, sowie die Patienten mit beiden Tumorentitäten (65,1\%; n=28), die nun häufiger einen Sonnenhut trugen. Bei den Melanompatienten waren es 
54,5\% $(n=43)$. Auch hier zeigte sich keine statistische Signifikanz $(p=0,158)$. Details finden sich in Tabelle 4.41 .

\begin{tabular}{|l|c|c|c|}
\hline & Melanom & $\begin{array}{l}\text { Nicht-melanozytäre } \\
\text { Tumoren }\end{array}$ & $\begin{array}{l}\text { Melanom und } \\
\text { Nicht-melanozytäre } \\
\text { Tumoren }\end{array}$ \\
\hline Trifft voll zu & $26,6 \%$ & $36,2 \%$ & $32,6 \%$ \\
\hline Trifft weitgehend zu & $16,5 \%$ & $19,9 \%$ & $20,9 \%$ \\
\hline Trifft ziemlich zu & $11,4 \%$ & $11,3 \%$ & $11,6 \%$ \\
\hline Trifft kaum zu & $19,0 \%$ & $12,8 \%$ & $18,6 \%$ \\
\hline Trifft gar nicht zu & $26,6 \%$ & $19,9 \%$ & $16,3 \%$ \\
\hline P-Wert & \multicolumn{3}{|c|}{0,158} \\
\hline
\end{tabular}

Tabelle 4.41: ...trage ich regelmäßig einen Sonnenhut (Auswertung nach der Grunddiagnose)

Sonnenbrillen wurden vor allem in der Gruppe, die unter beiden Tumorentitäten litt, vermehrt getragen (65,1\%; n=28). Bei den anderen beiden Gruppen waren es jeweils 55,7\%, die ihr Verhalten änderten - bei nicht-melanozytären Tumoren entsprach dies $n=78$; bei melanozytären Tumoren $\mathrm{n}=44$. Der Unterschied zeigte keine Signifikanz $(\mathrm{p}=0,76)$. Details finden sich in Tabelle 4.42.

\begin{tabular}{|l|c|c|c|}
\hline & Melanom & $\begin{array}{l}\text { Nicht-melanozytäre } \\
\text { Tumoren }\end{array}$ & $\begin{array}{l}\text { Melanom und } \\
\text { Nicht-melanozytäre } \\
\text { Tumoren }\end{array}$ \\
\hline Trifft voll zu & $22,8 \%$ & $25,0 \%$ & $18,6 \%$ \\
\hline Trifft weitgehend zu & $21,5 \%$ & $16,4 \%$ & $30,2 \%$ \\
\hline Trifft ziemlich zu & $11,4 \%$ & $14,3 \%$ & $16,3 \%$ \\
\hline Trifft kaum zu & $21,5 \%$ & $20,7 \%$ & $20,9 \%$ \\
\hline Trifft gar nicht zu & $22,8 \%$ & $23,6 \%$ & $14,0 \%$ \\
\hline P-Wert & \multicolumn{2}{|}{0,760} \\
\hline
\end{tabular}

Tabelle 4.42: ...trage ich regelmäßig eine Sonnenbrille (Auswertung nach der Grunddiagnose)

\subsubsection{Auswertung nach dem Schulabschluss}

Mit $71,7 \% \quad(n=142)$ benutzten die Befragten mit niedrigerem Qualifikationsniveau nach Bekanntwerden ihrer Tumorerkrankung etwas häufiger Sonnenschutzcremes als die mit höherem Qualifikationsniveau (58,9\%; $n=36)$. Details finden sich in Tabelle 4.43. Der Unterschied zwischen den Gruppen war nicht signifikant $(p=0,173)$. 
Bei der Frage nach einem textilen Schutz mittels Sonnenhut antworteten von den Befragten mit höherer Qualifikation etwas mehr (68,9\%; n=42) mit „trifft voll zu“, „trifft weitgehend“ zu oder „trifft ziemlich zu“ als dies bei den Befragten mit niedrigerer Qualifikation (60,6\%; $n=120$ ) der Fall war (Tabelle 4.44). Dieser Unterschied war jedoch nicht signifikant ( $\mathrm{p}=0,304)$.

Ein ähnliches Verteilungsmuster, mit 56,3\% $(n=111)$ bei Patienten mit niedrigerem Bildungsniveau gegenüber 60,7\% ( $\mathrm{n}=37)$ bei Patienten mit höherem Bildungsniveau, ergab die Frage nach dem Tragen einer Sonnenbrille nach Diagnosestellung (Tabelle 4.45). Der Unterschied war wiederum nicht signifikant $(\mathrm{p}=0,482)$.

\begin{tabular}{|l|l|l|}
\hline & bis einschließlich mittlere Reife & ab Fachhochschulreife \\
\hline Trifft voll zu & $36,4 \%$ & $34,3 \%$ \\
\hline Trifft weitgehend zu & $23,7 \%$ & $13,1 \%$ \\
\hline Trifft ziemlich zu & $11,6 \%$ & $11,5 \%$ \\
\hline Trifft kaum zu & $13,6 \%$ & $18,0 \%$ \\
\hline Trifft gar nicht zu & $14,6 \%$ & $23,0 \%$ \\
\hline P-Wert & & \\
\hline
\end{tabular}

Tabelle 4.43: ...benutze ich häufiger als früher Sonnenschutzcremes mit Lichtschutzfaktor (Auswertung nach Schulabschluss)

\begin{tabular}{|l|l|l|}
\hline & bis einschließlich mittlere Reife & ab Fachhochschulreife \\
\hline Trifft voll zu & $30,8 \%$ & $37,7 \%$ \\
\hline Trifft weitgehend zu & $20,2 \%$ & $16,4 \%$ \\
\hline Trifft ziemlich zu & $9,6 \%$ & $14,8 \%$ \\
\hline Trifft kaum zu & $16,7 \%$ & $13,1 \%$ \\
\hline Trifft gar nicht zu & $22,7 \%$ & $18,0 \%$ \\
\hline P-Wert & & \\
\hline
\end{tabular}

Tabelle 4.44: ...trage ich regelmäßig einen Sonnenhut (Auswertung nach Schulabschluss)

\begin{tabular}{|l|l|l|}
\hline & bis einschließlich mittlere Reife & ab Fachhochschulreife \\
\hline Trifft voll zu & $22,3 \%$ & $24,6 \%$ \\
\hline Trifft weitgehend zu & $20,8 \%$ & $19,7 \%$ \\
\hline Trifft ziemlich zu & $13,2 \%$ & $16,4 \%$ \\
\hline Trifft kaum zu & $20,3 \%$ & $23,0 \%$ \\
\hline Trifft gar nicht zu & $23,4 \%$ & $16,4 \%$ \\
\hline P-Wert & & \\
\hline
\end{tabular}

Tabelle 4.45: ...trage ich regelmäßig eine Sonnenbrille (Auswertung nach dem Schulabschluss) 


\subsubsection{Auswertung nach dem Familienstand}

70,4\% ( $\mathrm{n=138)}$ der Patienten, die mit Partner lebten, benutzen häufiger Sonnenschutzcremes als noch vor Bekanntwerden der Tumorerkrankung. Bei den allein lebenden Patienten waren es mit 64,1\% ( $n=41)$ etwas weniger (Tabelle 4.46). Der Unterschied beider Gruppen war nicht signifikant $(\mathrm{p}=0,281)$.

\begin{tabular}{|l|l|l|}
\hline & mit Partner & ohne Partner \\
\hline Trifft voll zu & $36,7 \%$ & $32,8 \%$ \\
\hline Trifft weitgehend zu & $23,0 \%$ & $17,2 \%$ \\
\hline Trifft ziemlich zu & $10,7 \%$ & $14,1 \%$ \\
\hline Trifft kaum zu & $14,3 \%$ & $15,6 \%$ \\
\hline Trifft gar nicht zu & $15,3 \%$ & $20,3 \%$ \\
\hline P-Wert & & \\
\hline
\end{tabular}

Tabelle 4.46: ...benutze ich häufiger als früher Sonnenschutzcremes mit Lichtschutzfaktor (Auswertung nach Familienstand)

Das häufigere Tragen eines Sonnenhutes wurde mit 64,3\% ( $n=126)$ eher von den Patienten mit Partner praktiziert. Bei Patienten ohne Partner waren es 57,8\% ( $\mathrm{n}=37)$. Details sind Tabelle 4.47 bzw. Abbildung 4.26 zu entnehmen. Dieser Unterschied war signifikant $(\mathrm{p}=0,042)$.

Bei Patienten mit Partner trugen 59,1\% ( $n=115)$, bei Patienten ohne Partner 53,1\% ( $n=34)$ häufiger eine Sonnenbrille als vor Bekanntwerden ihrer Hauttumorerkrankung. Das Ergebnis zeigte keine statistische Signifikanz ( $\mathrm{p}=0,518$ ). Details sind Tabelle $4.48 \mathrm{zu}$ entnehmen.

\begin{tabular}{|l|l|l|}
\hline & mit Partner & ohne Partner \\
\hline Trifft voll zu & $35,2 \%$ & $25,0 \%$ \\
\hline Trifft weitgehend zu & $19,9 \%$ & $17,2 \%$ \\
\hline Trifft ziemlich zu & $9,2 \%$ & $15,6 \%$ \\
\hline Trifft kaum zu & $17,9 \%$ & $9,4 \%$ \\
\hline Trifft gar nicht zu & $17,9 \%$ & $32,8 \%$ \\
\hline P-Wert & & \\
\hline
\end{tabular}

Tabelle 4.47: ...trage ich regelmäßig einen Sonnenhut (Auswertung nach Familienstand) 


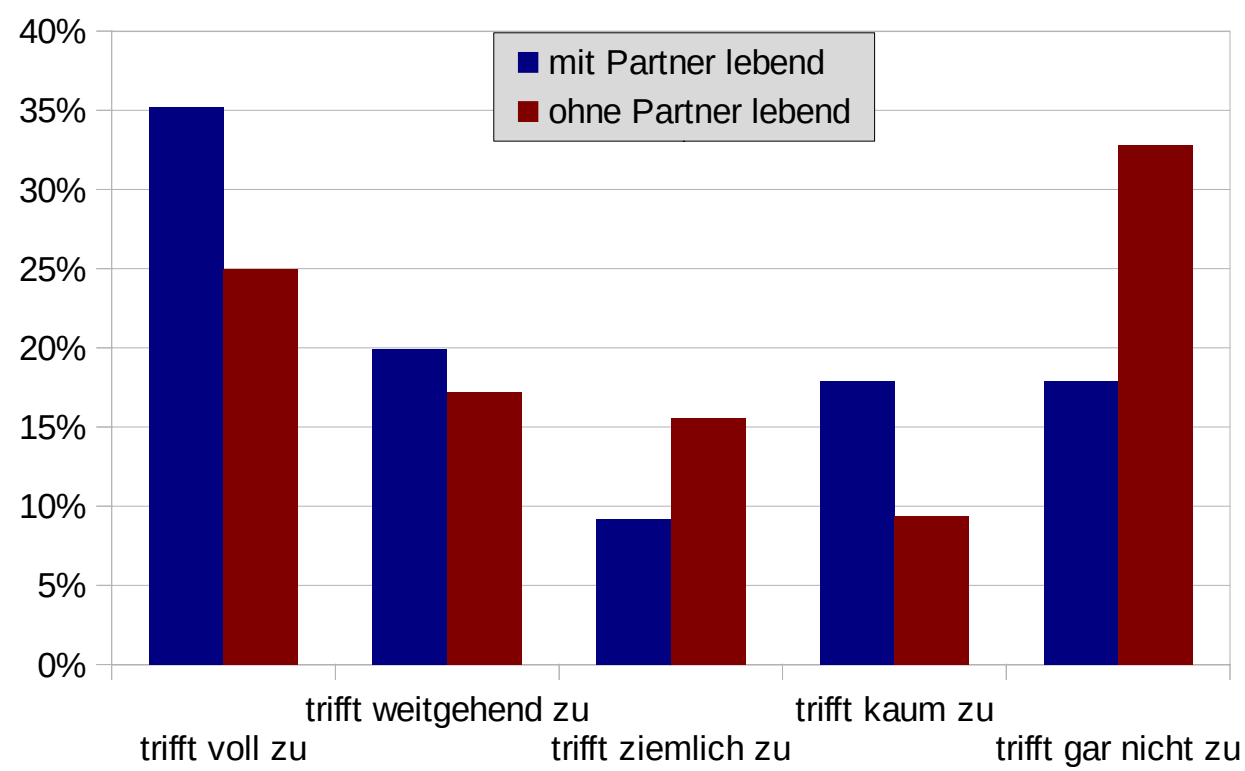

Abbildung 4.26: ...trage ich regelmäßig einen Sonnenhut (Auswertung nach Familienstand)

\begin{tabular}{|l|l|l|}
\hline & mit Partner & ohne Partner \\
\hline Trifft voll zu & $22,1 \%$ & $26,6 \%$ \\
\hline Trifft weitgehend zu & $22,1 \%$ & $15,6 \%$ \\
\hline Trifft ziemlich zu & $14,9 \%$ & $10,9 \%$ \\
\hline Trifft kaum zu & $22,0 \%$ & $17,2 \%$ \\
\hline Trifft gar nicht zu & $19,0 \%$ & $29,7 \%$ \\
\hline P-Wert & & \\
\hline
\end{tabular}

Tabelle 4.48: ...trage ich regelmäßig eine Sonnenbrille (Auswertung nach Familienstand)

\subsubsection{Auswertung nach dem Alter}

Mit 79,3\% (n=69) waren es vor allem die jüngeren Patienten die nach Diagnosestellung vermehrt Sonnencremes auf trugen. Bei den älteren Patienten waren es mit 63,6\% $(n=112)$ deutlich weniger. Details hierzu finden sich in Tabelle 4.49 bzw. Abbildung 4.27. Dieser Unterschied war statistisch signifikant $(\mathrm{p}=0,003)$. 


\begin{tabular}{|l|l|l|}
\hline & Unter 65 & Über 66 \\
\hline Trifft voll zu & $46,0 \%$ & $30,7 \%$ \\
\hline Trifft weitgehend zu & $23,0 \%$ & $21,0 \%$ \\
\hline Trifft ziemlich zu & $10,3 \%$ & $11,9 \%$ \\
\hline Trifft kaum zu & $10,3 \%$ & $16,5 \%$ \\
\hline Trifft gar nicht zu & $10,3 \%$ & $19,9 \%$ \\
\hline P-Wert & & \\
\hline
\end{tabular}

Tabelle 4.49: ...benutze ich häufiger als früher Sonnenschutzcremes mit Lichtschutzfaktor (Auswertung nach Alter)

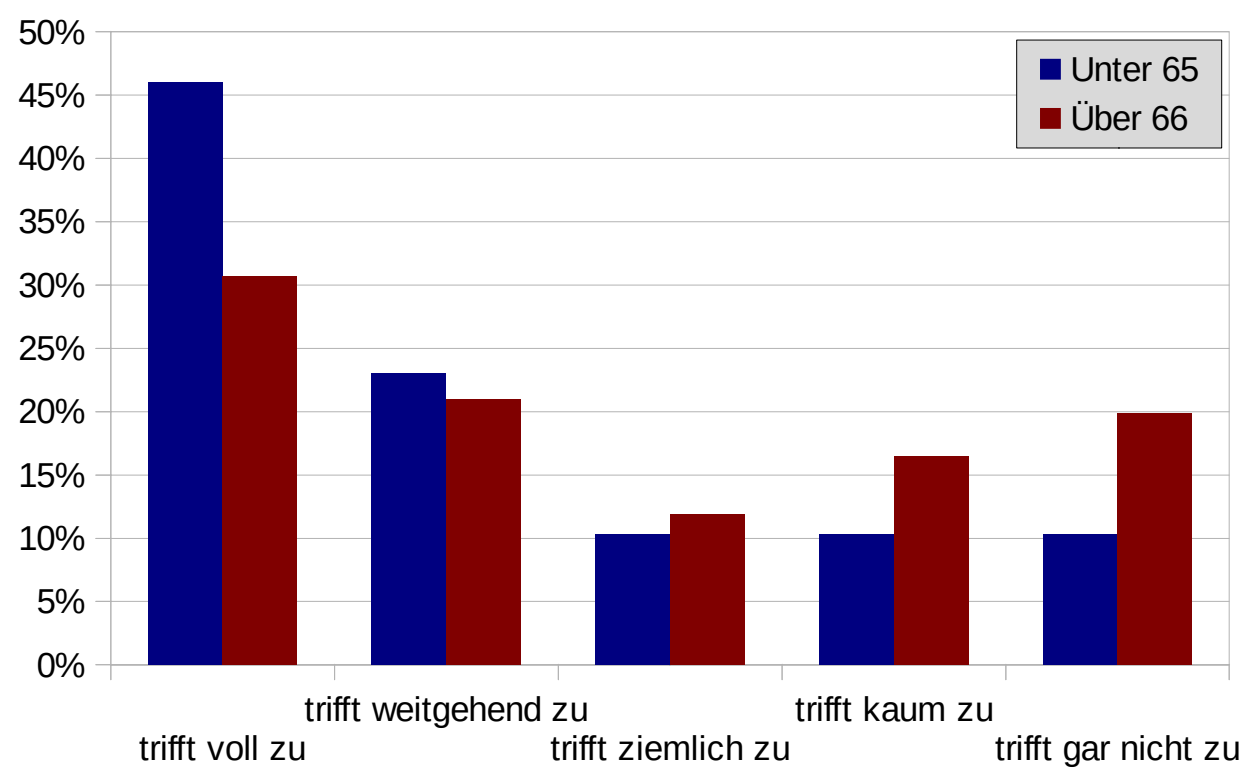

Abbildung 4.27: ...benutze ich häufiger als früher Sonnenschutzcremes mit Lichtschutzfaktor (Auswertung nach Alter)

Bei der Frage, ob nach Bekanntwerden der Hautkrebsdiagnose häufiger ein Sonnenhut getragen wurde, zeigte sich eine gegenläufige Tendenz. Hier waren es mit 68,8\% ( $n=121)$ vor allem die älteren Patienten, die die Frage mit „trifft voll zu“, „trifft weitgehend zu“ und „trifft ziemlich zu“ beantworteten. Bei den jüngeren Patienten waren dies nur 51,7\% $(n=45)$. Der Unterschied ergab eine statistische Signifikanz (p=0,002). Details finden sich in Tabelle 4.50 bzw. Abbildung 4.28. 


\begin{tabular}{|l|l|l|}
\hline & Unter 65 & Über 66 \\
\hline Trifft voll zu & $20,7 \%$ & $38,6 \%$ \\
\hline Trifft weitgehend zu & $16,1 \%$ & $20,5 \%$ \\
\hline Trifft ziemlich zu & $14,9 \%$ & $9,7 \%$ \\
\hline Trifft kaum zu & $23,0 \%$ & $11,9 \%$ \\
\hline Trifft gar nicht zu & $25,3 \%$ & $19,3 \%$ \\
\hline P-Wert & & \\
\hline
\end{tabular}

Tabelle 4.50: ...trage ich regelmäßig einen Sonnenhut (Auswertung nach Alter)

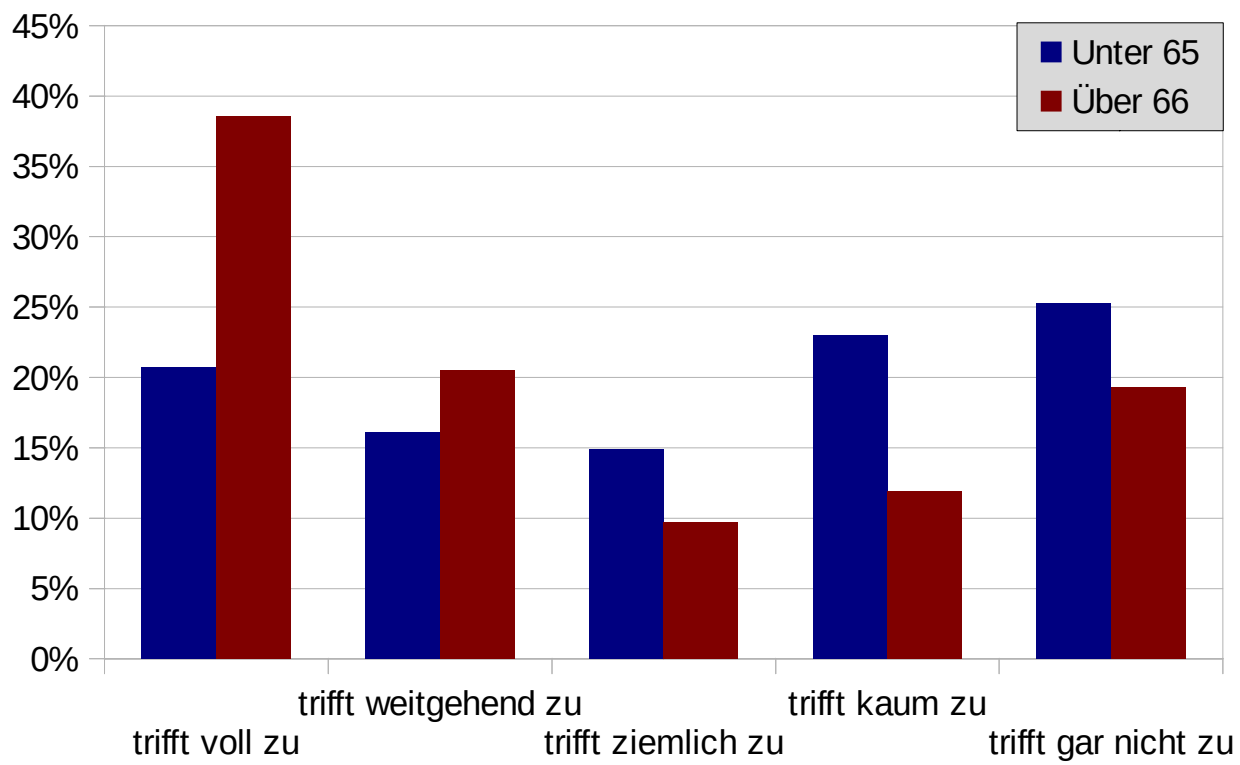

Abbildung 4.28: ...trage ich regelmäßig einen Sonnenhut (Auswertung nach Alter)

Die Frage, ob nach Bekanntwerden der Hauttumorerkrankung vermehrt Sonnenbrillen getragen wurden, ergab zwischen beiden Gruppen keine statistisch signifikanten Unterschiede $(p=0,294)$. Unter den Patienten, die ihr Verhalten geändert hatten, waren es bei den jüngeren mit $63,2 \%(n=55)$ etwas mehr, bei den älteren mit 54,3\% (=95) etwas weniger. Detaillierte Daten dazu werden in Tabelle 4.51 dargestellt. 


\begin{tabular}{|l|l|l|}
\hline & Unter 65 & Über 66 \\
\hline Trifft voll zu & $23,0 \%$ & $23,4 \%$ \\
\hline Trifft weitgehend zu & $23,0 \%$ & $18,9 \%$ \\
\hline Trifft ziemlich zu & $17,2 \%$ & $12,0 \%$ \\
\hline Trifft kaum zu & $20,7 \%$ & $21,1 \%$ \\
\hline Trifft gar nicht zu & $16,1 \%$ & $24,6 \%$ \\
\hline P-Wert & & \\
\hline
\end{tabular}

Tabelle 4.51: ...trage ich regelmäßig eine Sonnenbrille (Auswertung nach Alter)

\subsubsection{Urlaubsverhalten}

Die Frage, ob das Urlaubsverhalten geändert wurde (die Patienten also bspw. keinen Strandurlaub mehr machten) wurde von 56,5\% ( $\mathrm{n}=144)$ der Befragten positiv beantwortet. Die restlichen 43,5\% ( $\mathrm{n}=111$ ) antworteten mit „trifft kaum zu“ bzw. „trifft gar nicht zu“ (Abbildung 4.29). 8 Patienten ließen diese Frage unbeantwortet.

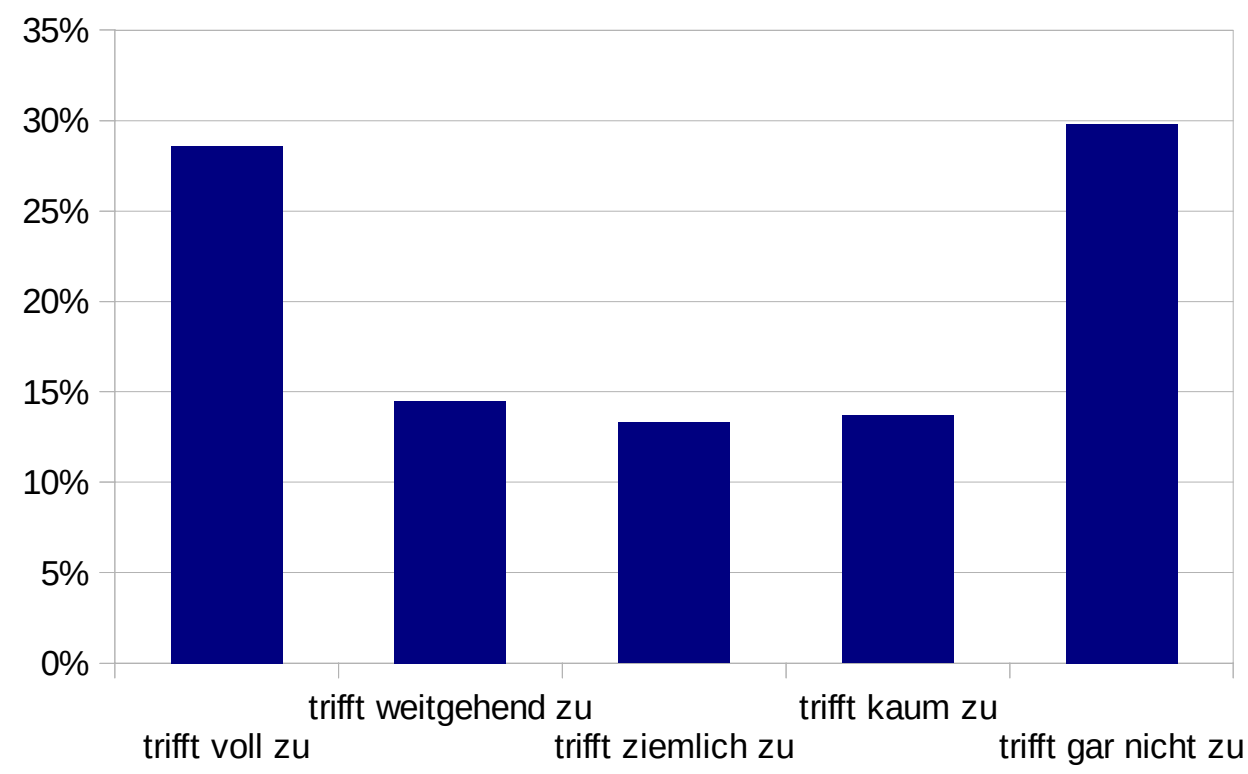

Abbildung 4.29: ...habe ich mein Urlaubsverhalten geändert

\subsubsection{Geschlechtsspezifische Auswertung}

Bei den Männern veränderten 58,4\% (n=94) ihr Verhalten, bei den Frauen waren es mit 53,2\% $(n=50)$ weniger. Eine statistische Signifikanz dieses Unterschieds bestand nicht $(p=0,701)$. Details finden sich in Tabelle 4.52. 


\begin{tabular}{|l|c|c|}
\hline & m & w \\
\hline Trifft voll zu & $27,3 \%$ & $30,9 \%$ \\
\hline Trifft weitgehend zu & $16,8 \%$ & $10,6 \%$ \\
\hline Trifft ziemlich zu & $14,3 \%$ & $11,7 \%$ \\
\hline Trifft kaum zu & $13,7 \%$ & $13,8 \%$ \\
\hline Trifft gar nicht zu & $28,0 \%$ & $33,0 \%$ \\
\hline P-Wert & & \\
\hline
\end{tabular}

Tabelle 4.52: ...habe ich mein Urlaubsverhalten geändert (geschlechtsspezifische Auswertung)

\subsubsection{Auswertung nach der Grunddiagnose}

Auch bei der Auswertung nach der Grunddiagnose gab es keine signifikanten Unterschiede ( $\mathrm{p}=$ 0,274). 61,1\% $(n=47)$ der Melanompatienten, bzw. 62,8\% $(n=70)$ der Patienten mit einer melanozytären sowie einer nicht-melanozytären Grunddiagnose bestätigten, ihr Urlaubsverhalten überdacht zu haben. Unter den Patienten mit nicht-melanozytärem Tumor erklärten dies nur 51,9\% ( $n=27)$. Details finden sich in Tabelle 4.53.

\begin{tabular}{|l|c|c|c|}
\hline & Melanom & $\begin{array}{l}\text { Nicht-melanozytäre } \\
\text { Tumoren }\end{array}$ & $\begin{array}{l}\text { Melanom und } \\
\text { Nicht-melanozytäre } \\
\text { Tumoren }\end{array}$ \\
\hline Trifft voll zu & $28,6 \%$ & $26,7 \%$ & $34,9 \%$ \\
\hline Trifft weitgehend zu & $14,3 \%$ & $13,3 \%$ & $18,6 \%$ \\
\hline Trifft ziemlich zu & $18,2 \%$ & $11,9 \%$ & $9,3 \%$ \\
\hline Trifft kaum zu & $13,0 \%$ & $14,1 \%$ & $14,0 \%$ \\
\hline Trifft gar nicht zu & $26,0 \%$ & $34,1 \%$ & $23,3 \%$ \\
\hline P-Wert & \multicolumn{2}{|}{0,274} \\
\hline
\end{tabular}

Tabelle 4.53: ...habe ich mein Urlaubsverhalten geändert (Auswertung nach der Grunddiagnose)

\subsubsection{Auswertung nach dem Schulabschluss}

Bei der Differenzierung gemäß Schulabschluss zeigten sich ähnliche Ergebnisse ohne signifikanten Unterschied $(p=0,122)$. In beiden Gruppen antworteten etwas über die Hälfte, ihr Urlaubsverhalten vollständig oder zumindest etwas verändert zu haben (niedrigeres Qualifikationsniveau: 58,5\% ( $n=113)$; höheres Qualifikationsniveau: 50,8\% $(n=30)$ ). Details finden sich in Tabelle 4.54. 


\begin{tabular}{|l|l|l|}
\hline & bis einschließlich mittlere Reife & ab Fachhochschulreife \\
\hline Trifft voll zu & $31,6 \%$ & $20,3 \%$ \\
\hline Trifft weitgehend zu & $14,5 \%$ & $13,6 \%$ \\
\hline Trifft ziemlich zu & $12,4 \%$ & $16,9 \%$ \\
\hline Trifft kaum zu & $13,5 \%$ & $15,3 \%$ \\
\hline Trifft gar nicht zu & $28,0 \%$ & $33,9 \%$ \\
\hline P-Wert & & \\
\hline
\end{tabular}

Tabelle 4.54: „..habe ich mein Urlaubsverhalten geändert (Auswertung nach Schulabschluss)

\subsubsection{Auswertung nach dem Familienstand}

Ähnliche Werte ergaben sich bei der Aufteilung nach dem Familienstand. Die Patienten mit Partner waren zu 58,0\% ( $n=112)$ bereit, ihr Urlaubsverhalten ganz oder teilweise zu ändern. Bei Patienten ohne Partner waren es mit 53,4\% (n=32) etwas weniger (Tabelle 4.55). Der Unterschied ergab keine statistische Signifikanz $(\mathrm{p}=0,579)$.

\begin{tabular}{|l|l|l|}
\hline & mit Partner & ohne Partner \\
\hline Trifft voll zu & $29,5 \%$ & $26,7 \%$ \\
\hline Trifft weitgehend zu & $13,0 \%$ & $20,0 \%$ \\
\hline Trifft ziemlich zu & $15,5 \%$ & $6,7 \%$ \\
\hline Trifft kaum zu & $14,5 \%$ & $11,7 \%$ \\
\hline Trifft gar nicht zu & $27,5 \%$ & $35,0 \%$ \\
\hline P-Wert & & \\
\hline
\end{tabular}

Tabelle 4.55: ...habe ich mein Urlaubsverhalten geändert (Auswertung nach Familienstand)

\subsubsection{Auswertung nach dem Alter}

Die Frage, ob das Urlaubsverhalten nach Bekanntwerden der Tumorerkrankung verändert wurde, beantworteten 52,3\% ( $n=44)$ der jüngeren und 58,4\% $(n=100)$ der älteren Patienten mit „, trifft voll zu“, „trifft weitgehend“ zu oder „trifft ziemlich zu“ (Tabelle 4.56). Der Unterschied ergab keine statistische Signifikanz $(\mathrm{p}=0,121)$. 


\begin{tabular}{|l|l|l|}
\hline & Unter 65 & Über 66 \\
\hline Trifft voll zu & $20,2 \%$ & $32,7 \%$ \\
\hline Trifft weitgehend zu & $11,9 \%$ & $15,8 \%$ \\
\hline Trifft ziemlich zu & $20,2 \%$ & $9,9 \%$ \\
\hline Trifft kaum zu & $17,9 \%$ & $11,7 \%$ \\
\hline Trifft gar nicht zu & $29,8 \%$ & $29,8 \%$ \\
\hline P-Wert & & \\
\hline
\end{tabular}

Tabelle 4.56: ...habe ich mein Urlaubsverhalten geändert (Auswertung nach Alter)

\subsubsection{Freizeitverhalten}

Es wurde geprüft, ob die Befragten nach der Diagnosestellung bereit waren, zu vermeiden, das Haus in den Mittagsstunden (also bei voller Sonneneinstrahlung) zu verlassen. Die Frage wurde von 80,2\% (n=207) mit „trifft voll zu“, „trifft weitgehend“ zu oder „trifft ziemlich zu“ beantwortet. Nur 19,8\% ( $n=51)$ hatten ihr Verhalten nicht oder kaum geändert. Eine graphische Darstellung findet sich in Abbildung 4.30. 5 Patienten beantworteten diese Frage nicht.

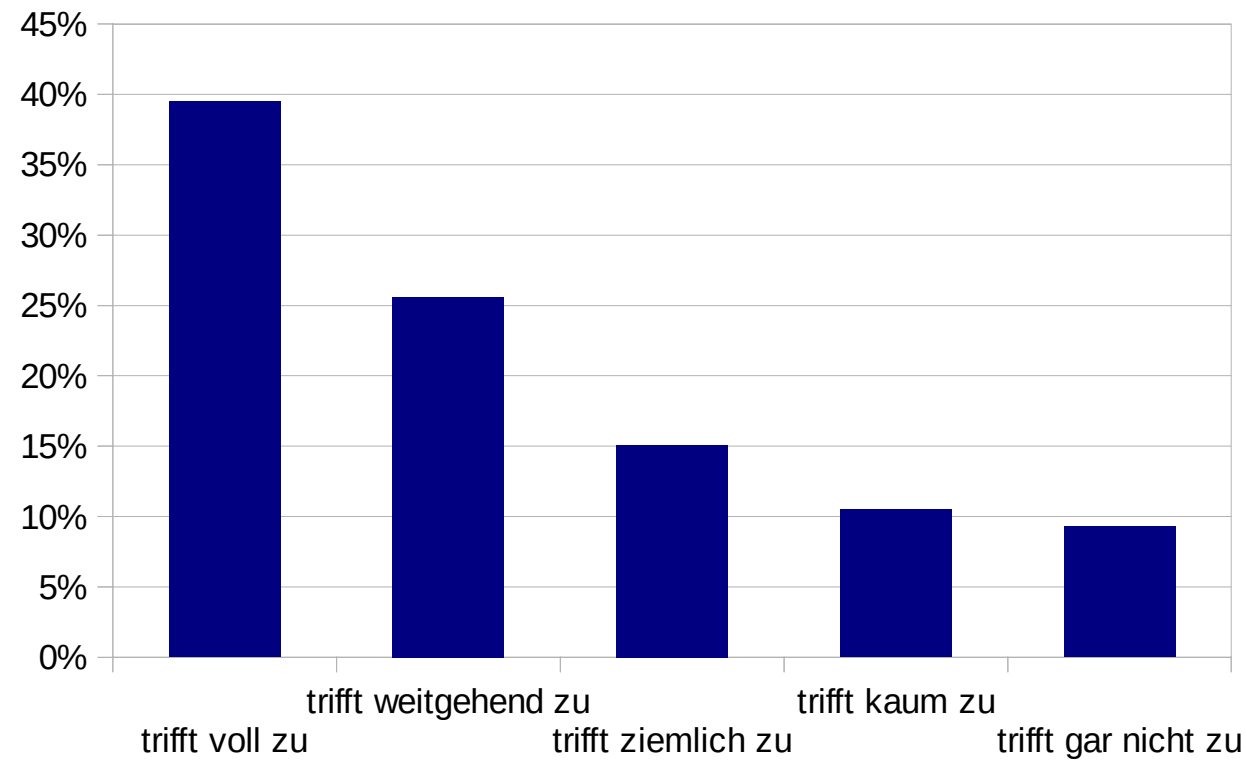

Abbildung 4.30: ...vermeide ich es in den Mittagsstunden, bei voller Sonneneinstrahlung, das Haus zu verlassen

Die Frage, ob die Patienten ihr Freizeitverhalten (weniger Freibadbesuche, Radtouren) verändert hätten, beantworteten 45,6\% (n=118) mit „trifft kaum“ bzw. „trifft gar nicht zu“. 54,4\% (n=141) hätten ihr Verhalten geändert (Abbildung 4.31). Diese Frage wurde von insgesamt 4 Patienten unbeantwortet gelassen. 


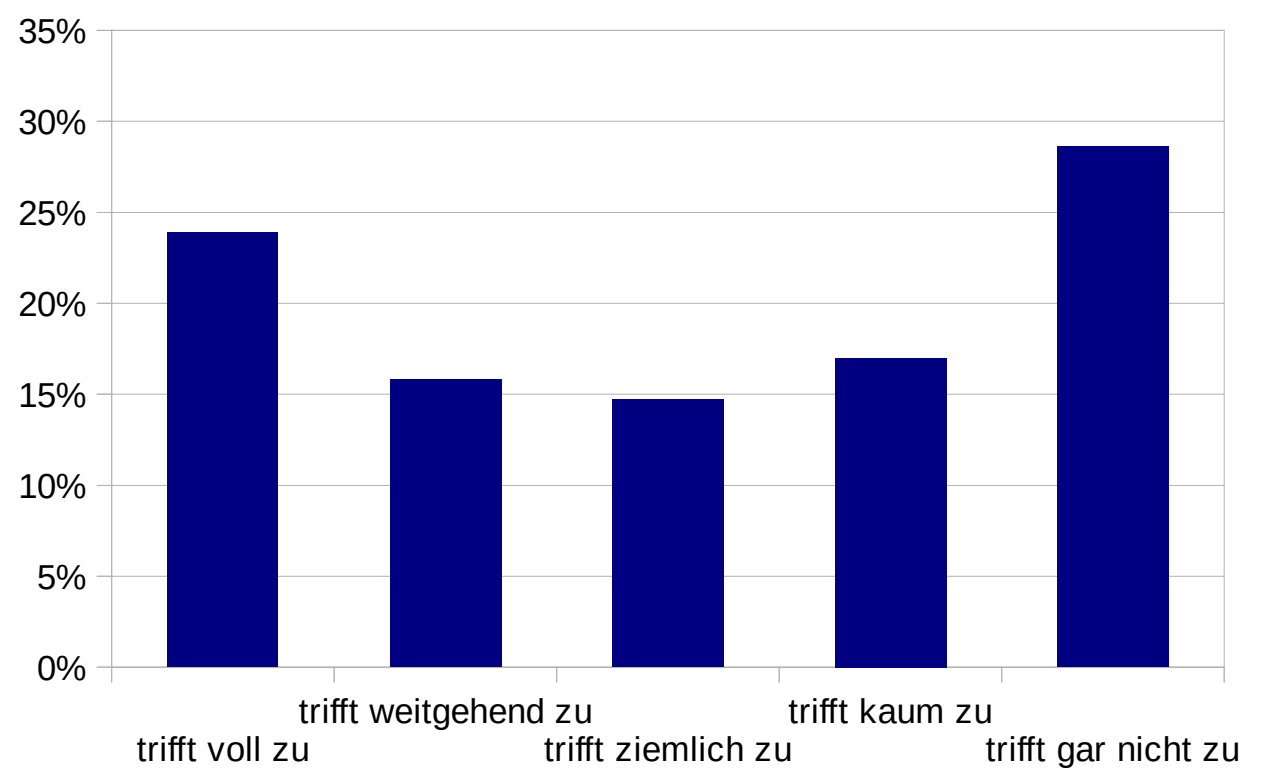

Abbildung 4.31: ...habe ich mein Freizeitverhalten (bspw. Freibadbesuche, Radtouren) verändert

\subsubsection{Geschlechtsspezifische Auswertung}

Bei den Frauen antworteten 50\% ( $\mathrm{n}=49)$ auf die Frage, ob sie es vermieden, das Haus in den Mittagsstunden zu verlassen mit „trifft voll zu“. Demgegenüber antworteten nur 33,1\% (n=53) der Männer mit „trifft voll zu“. Der Anteil der Befragten, die ihr Verhalten völlig oder zum Teil veränderten hatten („trifft voll zu“, „trifft weitgehend“ zu oder „trifft ziemlich zu“) war bei den Männern mit 81,2\% ( $n=130)$ gegenüber 78,6\% $(n=77)$ bei den Frauen geringfügig höher (Abbildung 4.32). Die Unterschiede zeigten keine statistische Signifikanz ( $\mathrm{p}=0,198)$.

Bei der Frage nach der Änderung des Freizeitverhaltens zeigten sich keine signifikanten Unterschiede $(\mathrm{p}=0,543)$. Unter den Männern, die ihr Verhalten vollständig oder zum Teil geändert hatten, waren es 57,5\% (n=93), bei den Frauen waren es mit 49,4\% ( $n=48)$ weniger (Tabelle 4.57).

\begin{tabular}{|l|c|c|}
\hline & $\mathrm{m}$ & $\mathrm{w}$ \\
\hline Trifft voll zu & $24,1 \%$ & $23,7 \%$ \\
\hline Trifft weitgehend zu & $16,7 \%$ & $14,4 \%$ \\
\hline Trifft ziemlich zu & $16,7 \%$ & $11,3 \%$ \\
\hline Trifft kaum zu & $14,8 \%$ & $20,6 \%$ \\
\hline Trifft gar nicht zu & $27,8 \%$ & $29,9 \%$ \\
\hline P-Wert & & \\
\hline
\end{tabular}

Tabelle 4.57: ...habe ich mein Freizeitverhalten (bspw. Freibadbesuche, Radtouren) verändert (geschlechtsspezifische Auswertung) 


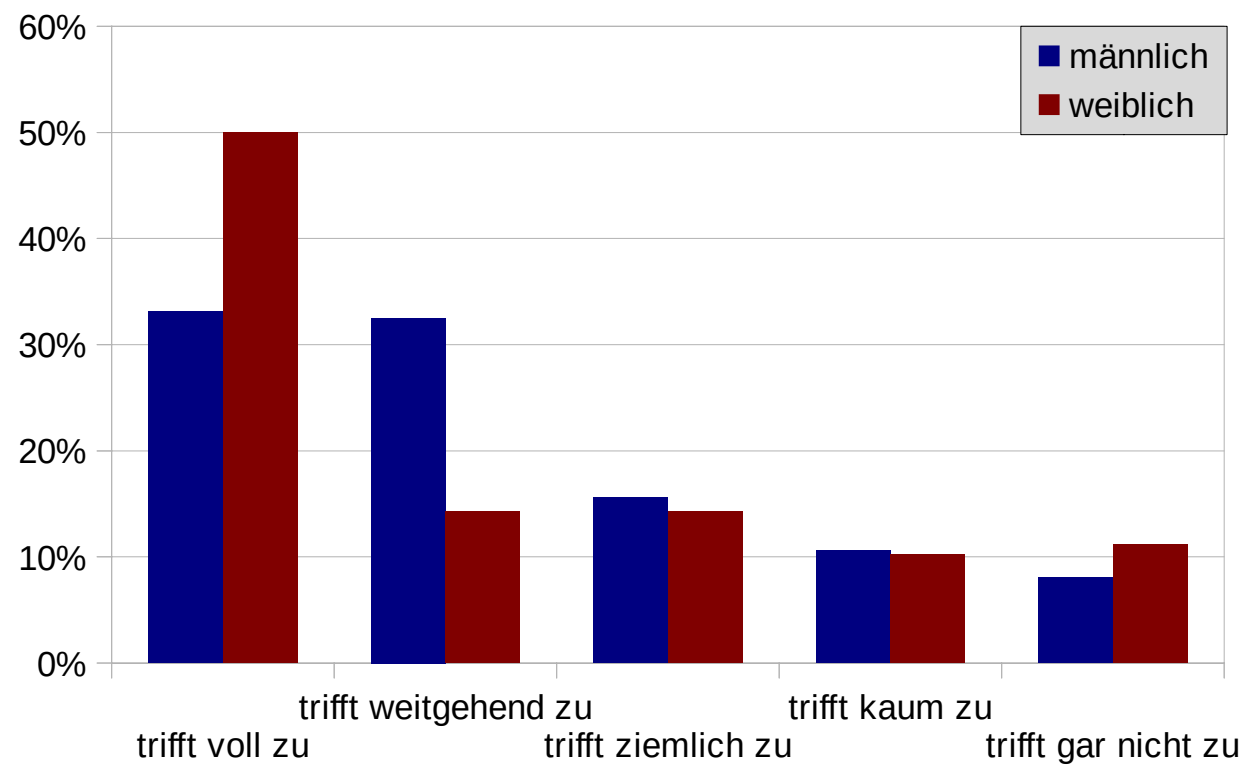

Abbildung 4.32: ...vermeide ich es in den Mittagsstunden, bei voller Sonneneinstrahlung, das Haus zu verlassen (geschlechtsspezifische Auswertung)

\subsubsection{Auswertung nach der Grunddiagnose}

Bei den Patienten mit nicht-melanozytärem Tumor $(83,9 \%$; $n=115)$, sowie Patienten mit beiden Tumorarten (81,4\%; $n=35)$ zeigte sich nach der Diagnose eine erhöhte Bereitschaft, das Haus in den Mittagsstunden nicht zu verlassen. Bei den Melanompatienten waren es 73,1\% ( $n=57)$. Details finden sich in Tabelle 4.58. Der Unterschied war statistisch nicht signifikant $(p=0,353)$.

\begin{tabular}{|l|c|c|c|}
\hline & Melanom & $\begin{array}{l}\text { Nicht-melanozytäre } \\
\text { Tumoren }\end{array}$ & $\begin{array}{l}\text { Melanom und } \\
\text { Nicht-melanozytäre } \\
\text { Tumoren }\end{array}$ \\
\hline Trifft voll zu & $37,2 \%$ & $40,9 \%$ & $39,5 \%$ \\
\hline Trifft weitgehend zu & $19,2 \%$ & $29,9 \%$ & $23,3 \%$ \\
\hline Trifft ziemlich zu & $16,7 \%$ & $13,1 \%$ & $18,6 \%$ \\
\hline Trifft kaum zu & $15,4 \%$ & $5,8 \%$ & $16,3 \%$ \\
\hline Trifft gar nicht zu & $11,5 \%$ & $10,2 \%$ & $2,3 \%$ \\
\hline P-Wert & \multicolumn{3}{|c|}{0,353} \\
\hline
\end{tabular}

Tabelle 4.58: ...vermeide ich es in den Mittagsstunden, bei voller Sonneneinstrahlung, das Haus zu verlassen (Auswertung nach der Grunddiagnose)

Die Frage, ob die Tumorerkrankung das Freizeitverhalten beeinflusst habe, wurde von allen Gruppen mit ähnlichen Werten beantwortet. Mit „trifft voll zu“, „trifft weitgehend zu“ und „trifft 
ziemlich zu“ beantworteten diese Frage unter den Melanompatienten 51,3\% ( $n=40)$, unter Patienten mit nicht-melanozytärem Tumor 54,4\% $(\mathrm{n}=75)$ und unter Patienten mit beiden Tumorentitäten 60,6\% ( $\mathrm{n}=26)$. Der Unterschied war statistisch nicht signifikant $(\mathrm{p}=0,314)$. Details finden such in Tabelle 4.59. Der Unterschied wies keine statistische Signifikanz auf ( $p=0,314)$.

\begin{tabular}{|l|c|c|c|}
\hline & Melanom & $\begin{array}{l}\text { Nicht-melanozytäre } \\
\text { Tumoren }\end{array}$ & $\begin{array}{l}\text { Melanom und } \\
\text { Nicht-melanozytäre } \\
\text { Tumoren }\end{array}$ \\
\hline Trifft voll zu & $19,2 \%$ & $23,9 \%$ & $32,6 \%$ \\
\hline Trifft weitgehend zu & $15,4 \%$ & $16,7 \%$ & $14,0 \%$ \\
\hline Trifft ziemlich zu & $16,7 \%$ & $13,8 \%$ & $14,0 \%$ \\
\hline Trifft kaum zu & $25,6 \%$ & $10,9 \%$ & $20,9 \%$ \\
\hline Trifft gar nicht zu & $23,1 \%$ & $34,8 \%$ & $18,6 \%$ \\
\hline P-Wert & \multicolumn{3}{|c}{0,314} \\
\hline
\end{tabular}

Tabelle 4.59: ...habe ich mein Freizeitverhalten (bspw. Freibadbesuche, Radtouren) verändert (Auswertung nach der Grunddiagnose)

\subsubsection{Auswertung nach dem Schulabschluss}

Auch bei der Unterteilung der Patienten gemäß Schulabschluss zeigten sich nur minimale Unterschiede ( $\mathrm{p}=0,147)$. 80,9\% $(\mathrm{n}=157)$ der Patienten mit niedrigerem Schulabschluss vis-à-vis 78,3\% ( $n=47)$ der Patienten mit höherem Schulabschluss vermieden es, das Haus zur Mittagszeit zu verlassen (Tabelle 4.60).

Bei den geringer ausgebildeten Patienten waren es 57\% ( $n=111)$, die Freibadbesuche und ähnliche Aktivitäten teilweise oder vollständig vermieden. Bei den Patienten mit höherer Qualifikation waren es 46,6\% $(n=28)$. Details finden sich in Tabelle 4.61. Statistisch signifikante Unterschiede gab es nicht $(\mathrm{p}=0,081)$.

\begin{tabular}{|l|l|l|}
\hline & bis einschließlich mittlere Reife & ab Fachhochschulreife \\
\hline Trifft voll zu & $42,8 \%$ & $28,3 \%$ \\
\hline Trifft weitgehend zu & $23,2 \%$ & $35,0 \%$ \\
\hline Trifft ziemlich zu & $14,9 \%$ & $15,0 \%$ \\
\hline Trifft kaum zu & $10,8 \%$ & $10,0 \%$ \\
\hline Trifft gar nicht zu & $8,2 \%$ & $11,7 \%$ \\
\hline P-Wert & & \\
\hline
\end{tabular}

Tabelle 4.60: ...vermeide ich es in den Mittagsstunden, bei voller Sonneneinstrahlung, das Haus zu verlassen (Auswertung nach Schulabschluss) 


\begin{tabular}{|l|l|l|}
\hline & bis einschließlich mittlere Reife & ab Fachhochschulreife \\
\hline Trifft voll zu & $26,2 \%$ & $18,3 \%$ \\
\hline Trifft weitgehend zu & $15,9 \%$ & $13,3 \%$ \\
\hline Trifft ziemlich zu & $14,9 \%$ & $15,0 \%$ \\
\hline Trifft kaum zu & $17,4 \%$ & $16,7 \%$ \\
\hline Trifft gar nicht zu & $25,6 \%$ & $36,7 \%$ \\
\hline P-Wert & & \\
\hline
\end{tabular}

Tabelle 4.61: ...habe ich mein Freizeitverhalten (bspw. Freibadbesuche, Radtouren) verändert (Auswertung nach Schulabschluss)

\subsubsection{Auswertung nach dem Familienstand}

Die Auswertung gemäß Familienstand ergab, dass nach der Diagnosestellung 79,2\% (n=153) der Patienten mit Partner, und 83,8\% ( $\mathrm{n}=52)$ der Patienten ohne Partner, das direkte Sonnenlicht zur Mittagszeit, teilweise oder ganz vermieden (Tabelle 4.62) Der Unterschied war nicht signifikant $(\mathrm{p}=0,522)$.

Bezüglich der Freizeitaktivitäten hatten 55,1\% ( $\mathrm{n}=107)$ der Patienten mit Partner ihr Verhalten verändert. Bei Patienten ohne Partner waren es 53,2\% $(n=33)$. Details finden sich in Tabelle 4.63. Der Unterschied war ohne Signifikanz ( $\mathrm{p}=0,465)$.

\begin{tabular}{|l|l|l|}
\hline & mit Partner & ohne Partner \\
\hline Trifft voll zu & $38,3 \%$ & $43,5 \%$ \\
\hline Trifft weitgehend zu & $26,9 \%$ & $22,6 \%$ \\
\hline Trifft ziemlich zu & $14,0 \%$ & $17,7 \%$ \\
\hline Trifft kaum zu & $11,4 \%$ & $8,1 \%$ \\
\hline Trifft gar nicht zu & $9,3 \%$ & $8,1 \%$ \\
\hline P-Wert & & \\
\hline
\end{tabular}

Tabelle 4.62: ...vermeide ich es in den Mittagsstunden, bei voller Sonneneinstrahlung, das Haus zu verlassen (Auswertung nach Familienstand) 


\begin{tabular}{|l|l|l|}
\hline & mit Partner & ohne Partner \\
\hline Trifft voll zu & $24,7 \%$ & $22,6 \%$ \\
\hline Trifft weitgehend zu & $16,0 \%$ & $14,5 \%$ \\
\hline Trifft ziemlich zu & $14,4 \%$ & $16,1 \%$ \\
\hline Trifft kaum zu & $18,6 \%$ & $12,9 \%$ \\
\hline Trifft gar nicht zu & $26,3 \%$ & $33,9 \%$ \\
\hline P-Wert & & \\
\hline
\end{tabular}

Tabelle 4.63: ...habe ich mein Freizeitverhalten (bspw. Freibadbesuche, Radtouren) verändert (Auswertung nach Familienstand)

\subsubsection{Auswertung nach dem Alter}

84,3\% ( $n=144)$ der älteren Patienten vermieden es nach Diagnosestellung, das Haus in den Mittagsstunden zu verlassen. Bei den jüngeren Patienten waren es 72,4\% ( $n=63)$. Der Unterschied ergab mit $\mathrm{p}=0,013$ eine statistische Signifikanz. Details finden sich in Tabelle 4.64 und Abbildung 4.33.

Etwas über die Hälfte der jüngeren Patienten (55,8\%; n=48) und der älteren Patienten (53,8\%; n=93) veränderten ihr Freizeitverhalten (Tabelle 4.65). Es ergab sich keine statistische Signifikanz $(\mathrm{p}=0,862)$.

\begin{tabular}{|l|l|l|}
\hline & Unter 65 & Über 66 \\
\hline Trifft voll zu & $32,2 \%$ & $43,3 \%$ \\
\hline Trifft weitgehend zu & $21,8 \%$ & $27,5 \%$ \\
\hline Trifft ziemlich zu & $18,4 \%$ & $13,5 \%$ \\
\hline Trifft kaum zu & $14,9 \%$ & $8,2 \%$ \\
\hline Trifft gar nicht zu & $12,6 \%$ & $7,6 \%$ \\
\hline P-Wert & & \\
\hline
\end{tabular}

Tabelle 4.64: ...vermeide ich es in den Mittagsstunden, bei voller Sonneneinstrahlung, das Haus zu verlassen (Auswertung nach Alter) 


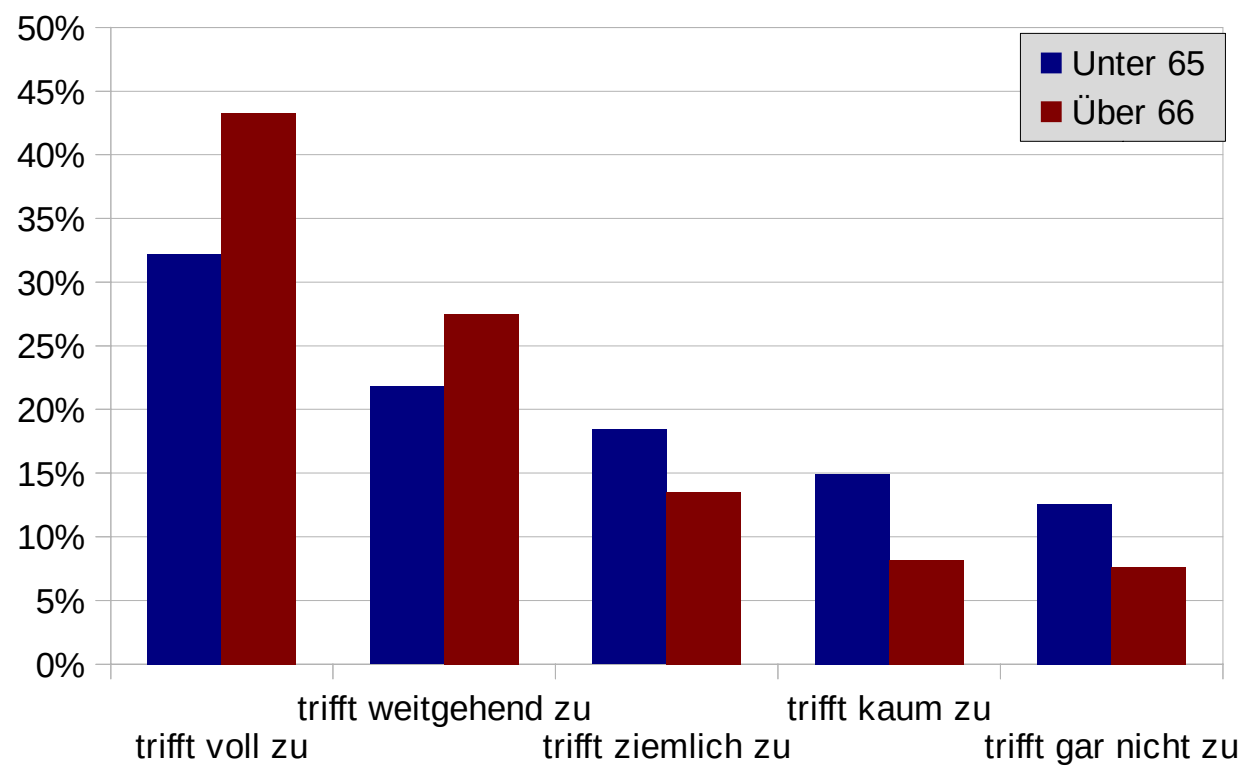

Abbildung 4.33: ...vermeide ich es in den Mittagsstunden, bei voller Sonneneinstrahlung, das Haus zu verlassen (Auswertung nach Alter)

\begin{tabular}{|l|l|l|}
\hline & Unter 65 & Über 66 \\
\hline Trifft voll zu & $17,4 \%$ & $27,2 \%$ \\
\hline Trifft weitgehend zu & $18,6 \%$ & $14,5 \%$ \\
\hline Trifft ziemlich zu & $19,8 \%$ & $12,1 \%$ \\
\hline Trifft kaum zu & $20,9 \%$ & $15,0 \%$ \\
\hline Trifft gar nicht zu & $23,3 \%$ & $31,2 \%$ \\
\hline P-Wert & & \\
\hline
\end{tabular}

Tabelle 4.65: ...habe ich mein Freizeitverhalten (bspw. Freibadbesuche, Radtouren) verändert (Auswertung nach Alter)

\subsubsection{Ernährung}

Das Ernährungsverhalten wurde ebenfalls erfragt. Ernährten sich die Patienten nach Bekanntwerden ihrer Tumorerkrankung gesünder und bewusster?

Über die Hälfte der Befragten (59,2\%; n=154) hatten ihr Ernährungsverhalten überdacht, hiervon antworteten 14,6\% (n=38) der Patienten mit „trifft voll zu“. Hingegen hatten 40,8\% (n=106) ihr Ernährungsverhalten nicht oder kaum verändert (Abbildung 4.34). Diese Frage blieb von insgesamt 3 Patienten unbeantwortet. 


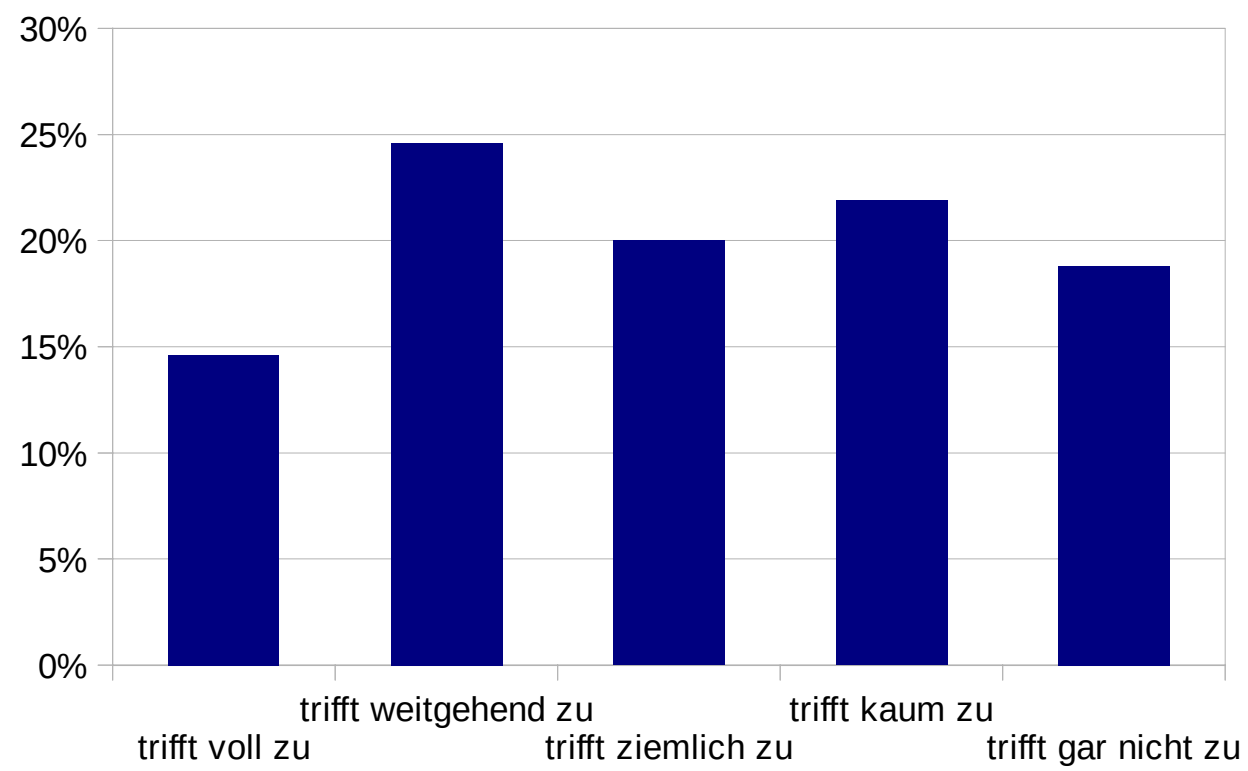

Abbildung 4.34: ...ernähre ich mich gesünder und bewusster

\subsubsection{Geschlechtsspezifische Auswertung}

Bei den Frauen änderten 63,3\% $(n=62)$ ihr Verhalten teilweise oder ganz. Sie ernährten sich gesünder als noch vor Bekanntwerden ihrer Tumorerkrankung. Hingegen waren es bei den Männern mit 56,8\% ( $n=92)$ etwas weniger (Tabelle 4.66). Eine statistische Signifikanz $(p=0,260)$ war jedoch nicht erkennbar.

\begin{tabular}{|l|c|c|}
\hline & m & w \\
\hline Trifft voll zu & $14,8 \%$ & $14,3 \%$ \\
\hline Trifft weitgehend zu & $21,6 \%$ & $29,6 \%$ \\
\hline Trifft ziemlich zu & $20,4 \%$ & $19,4 \%$ \\
\hline Trifft kaum zu & $22,2 \%$ & $21,4 \%$ \\
\hline Trifft gar nicht zu & $21,0 \%$ & $15,3 \%$ \\
\hline P-Wert & & \\
\hline
\end{tabular}

Tabelle 4.66: ...ernähre ich mich gesünder und bewusster (geschlechtsspezifische Auswertung)

\subsubsection{Auswertung nach Grunddiagnose}

Mit 60,8\% ( $n=48)$ waren es vor allem die Melanompatienten und mit 65,1\% $(n=28)$ die Patienten mit beiden Tumorentitäten, die sich nach der Diagnose teilweise oder ganz gesünder ernährten. Bei den Patienten mit nicht-melanozytärem Tumor waren es mit 56,5\% (n=78) etwas weniger die ihre Ernährung ganz oder zum Teil umstellten (Tabelle 4.67). Der Unterschied war nicht signifikant ( $p=0,353)$. 


\begin{tabular}{|l|c|c|c|}
\hline & Melanom & $\begin{array}{l}\text { Nicht-melanozytäre } \\
\text { Tumoren }\end{array}$ & $\begin{array}{l}\text { Melanom und } \\
\text { Nicht-melanozytäre } \\
\text { Tumoren }\end{array}$ \\
\hline Trifft voll zu & $22,8 \%$ & $10,9 \%$ & $11,6 \%$ \\
\hline Trifft weitgehend zu & $19,0 \%$ & $27,5 \%$ & $25,6 \%$ \\
\hline Trifft ziemlich zu & $19,0 \%$ & $18,1 \%$ & $27,9 \%$ \\
\hline Trifft kaum zu & $25,3 \%$ & $21,0 \%$ & $18,6 \%$ \\
\hline Trifft gar nicht zu & $13,9 \%$ & $22,5 \%$ & $16,3 \%$ \\
\hline P-Wert & \multicolumn{3}{|c|}{0,353} \\
\hline
\end{tabular}

Tabelle 4.67: ...ernähre ich mich gesünder und bewusster (Auswertung nach der Grunddiagnose)

\subsubsection{Auswertung nach dem Schulabschluss}

Bei den Befragten mit niedrigerer Qualifikation waren mehr (62,2\%; n=122) dazu bereit, sich nach der Diagnosestellung gesünder zu ernähren. Bei der Gruppe mit höherer Qualifikation waren es nur 50,0\% ( $\mathrm{n=30}$ ) die mit „trifft voll zu“, „trifft weitgehend zu“ oder „trifft ziemlich zu“ antworteten. Der Unterschied war nicht signifikant $(\mathrm{p}=0,118)$. Details finden sich in Tabelle 4.68.

\begin{tabular}{|l|l|l|}
\hline & bis einschließlich mittlere Reife & ab Fachhochschulreife \\
\hline Trifft voll zu & $14,8 \%$ & $15,0 \%$ \\
\hline Trifft weitgehend zu & $26,5 \%$ & $18,3 \%$ \\
\hline Trifft ziemlich zu & $20,9 \%$ & $16,7 \%$ \\
\hline Trifft kaum zu & $21,9 \%$ & $23,3 \%$ \\
\hline Trifft gar nicht zu & $15,8 \%$ & $26,7 \%$ \\
\hline P-Wert & & \\
\hline
\end{tabular}

Tabelle 4.68: ...ernähre ich mich gesünder und bewusster (Auswertung nach Schulabschluss)

\subsubsection{Auswertung nach dem Familienstand}

Bei der Differenzierung nach dem Familienstand zeigte sich, dass sowohl die Patienten mit Partner (59,5\%; n=116) als auch die ohne Partner (59,7\%; n=37) bereit waren ihr Ernährungsverhalten zu überdenken (Tabelle 4.69). Der Unterschied war nicht signifikant ( $\mathrm{p}=0,759)$. 


\begin{tabular}{|l|l|l|}
\hline & mit Partner & ohne Partner \\
\hline Trifft voll zu & $14,4 \%$ & $16,1 \%$ \\
\hline Trifft weitgehend zu & $24,6 \%$ & $24,2 \%$ \\
\hline Trifft ziemlich zu & $20,5 \%$ & $19,4 \%$ \\
\hline Trifft kaum zu & $21,5 \%$ & $24,2 \%$ \\
\hline Trifft gar nicht zu & $19,0 \%$ & $16,1 \%$ \\
\hline P-Wert & & \\
\hline
\end{tabular}

Tabelle 4.69: ...ernähre ich mich gesünder und bewusster (Auswertung nach Familienstand)

\subsubsection{Auswertung nach dem Alter}

Mit 56,2\% ( $n=49)$ bei den jüngeren, und 60,8\% ( $n=105)$ bei den älteren Patienten ernährten sich in beiden Gruppen ähnlich viele nach Diagnosestellung gesünder als noch davor (Tabelle 4.70). Der Unterschied war nicht signifikant $(\mathrm{p}=0,606)$.

\begin{tabular}{|l|l|l|}
\hline & Unter 65 & Über 66 \\
\hline Trifft voll zu & $14,9 \%$ & $14,5 \%$ \\
\hline Trifft weitgehend zu & $19,5 \%$ & $27,2 \%$ \\
\hline Trifft ziemlich zu & $21,8 \%$ & $19,1 \%$ \\
\hline Trifft kaum zu & $26,4 \%$ & $19,7 \%$ \\
\hline Trifft gar nicht zu & $17,2 \%$ & $19,7 \%$ \\
\hline P-Wert & & \\
\hline
\end{tabular}

Tabelle 4.70: ...ernähre ich mich gesünder und bewusster (Auswertung nach Alter)

\subsubsection{Alkoholgenuss}

60,9\% ( $n=157)$ der Befragten beantworteten die Frage, ob sie nach Bekanntwerden der Hauttumorerkrankung weniger Alkohol getrunken hatten mit „trifft voll zu“, „trifft weitgehend zu“ oder „trifft ziemlich zu“. Ein großer Teil (29,1\%; n=75) änderte das Trinkverhalten grundlegend und beantwortete die Frage mit „trifft voll zu“. Nur 39,1\% (n=101) veränderten ihr Verhalten kaum oder gar nicht (Abbildung 4.35). Diese Frage wurde von 5 Patienten nicht beantwortet. 


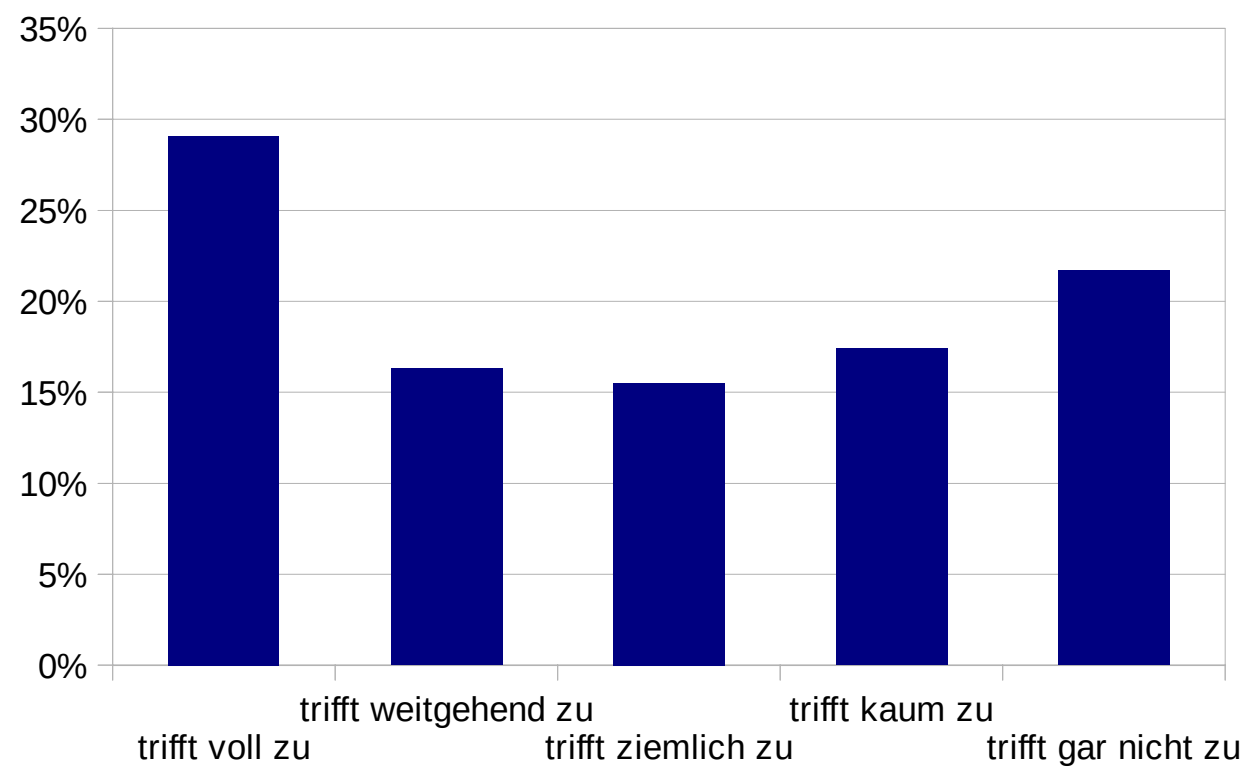

Abbildung 4.35: ...trinke ich weniger Alkohol

\subsubsection{Geschlechtsspezifische Auswertung}

Eine Unterteilung in Männer vis-à-vis Frauen zeigte ein ausgeglichenes Resultat ( $\mathrm{p}=0,81)$. Bei den Männern reduzierten 60,8\% ( $n=98)$, bei den Frauen 64,8\% (n=59) ihren Alkoholkonsum. 33\% $(n=32)$ der Frauen antworteten auf die Frage, ob weniger Alkohol getrunken würde mit „trifft voll zu“. Details finden sich in Tabelle 4.71.

\begin{tabular}{|l|c|c|}
\hline & m & w \\
\hline Trifft voll zu & $26,7 \%$ & $33,0 \%$ \\
\hline Trifft weitgehend zu & $16,1 \%$ & $16,5 \%$ \\
\hline Trifft ziemlich zu & $18,0 \%$ & $11,3 \%$ \\
\hline Trifft kaum zu & $20,5 \%$ & $12,4 \%$ \\
\hline Trifft gar nicht zu & $18,6 \%$ & $26,8 \%$ \\
\hline P-Wert & & \\
\hline
\end{tabular}

Tabelle 4.71: ...trinke ich weniger Alkohol (geschlechtsspezifische Auswertung)

\subsubsection{Auswertung nach Grunddiagnose}

Mit „trifft voll zu“, „trifft weitgehend zu“ oder „trifft ziemlich zu“ wurde die Frage, ob sie weniger Alkohol trinken würden, wie folgt beantwortet: Unter Patienten mit Melanom von 62,1\% (n=49); mit nicht-melanozytärem Tumor von 59,6\% ( $\mathrm{n}=81)$; und mit beiden Tumorentitäten von 62,8\% ( $n=27)$. Dem begrenzten Attribut „trifft voll zu“ pflichteten unter den Melanompatienten allerdings 
mit 20,3\% ( $n=16)$ wesentlich weniger Patienten bei. Trotzdem waren statistisch keine signifikanten Unterschiede festzustellen ( $\mathrm{p}=0,649)$. Details finden sich in Tabelle 4.72.

\begin{tabular}{|l|c|c|c|}
\hline & Melanom & $\begin{array}{l}\text { Nicht-melanozytäre } \\
\text { Tumoren }\end{array}$ & $\begin{array}{l}\text { Melanom und } \\
\text { Nicht-melanozytäre } \\
\text { Tumoren }\end{array}$ \\
\hline Trifft voll zu & $20,3 \%$ & $32,4 \%$ & $34,9 \%$ \\
\hline Trifft weitgehend zu & $24,1 \%$ & $14,0 \%$ & $9,3 \%$ \\
\hline Trifft ziemlich zu & $17,7 \%$ & $13,2 \%$ & $18,6 \%$ \\
\hline Trifft kaum zu & $13,9 \%$ & $19,9 \%$ & $16,3 \%$ \\
\hline Trifft gar nicht zu & $24,1 \%$ & $20,6 \%$ & $20,9 \%$ \\
\hline P-Wert & \multicolumn{2}{|}{0,649} \\
\hline
\end{tabular}

Tabelle 4.72: ...trinke ich weniger Alkohol (Auswertung nach der Grunddiagnose)

\subsubsection{Auswertung nach dem Schulabschluss}

Patienten mit niedrigerer Qualifikation (66\%; n=128) verringerten ihren Alkoholkonsum deutlich häufiger als solche mit höherer Qualifikation $(48,4 \%$; $=29)$. Die Frage, ob sie ihren Alkoholkonsum verringert hätten, beantworteten unter den Patienten mit niedrigerer Qualifikation sogar 33,5\% (n=65) mit „trifft voll zu“. Der sich daraus ergebende Unterschied war statistisch signifikant $(\mathrm{p}=0,02)$. Details finden sich in Tabelle 4.73 und Abbildung 4.36.

\begin{tabular}{|l|l|l|}
\hline & bis einschließlich mittlere Reife & ab Fachhochschulreife \\
\hline Trifft voll zu & $33,5 \%$ & $16,7 \%$ \\
\hline Trifft weitgehend zu & $15,5 \%$ & $20,0 \%$ \\
\hline Trifft ziemlich zu & $17,0 \%$ & $11,7 \%$ \\
\hline Trifft kaum zu & $13,9 \%$ & $26,7 \%$ \\
\hline Trifft gar nicht zu & $20,1 \%$ & $25,0 \%$ \\
\hline P-Wert & & \\
\hline
\end{tabular}

Tabelle 4.73: ...trinke ich weniger Alkohol (Auswertung nach Schulabschluss) 


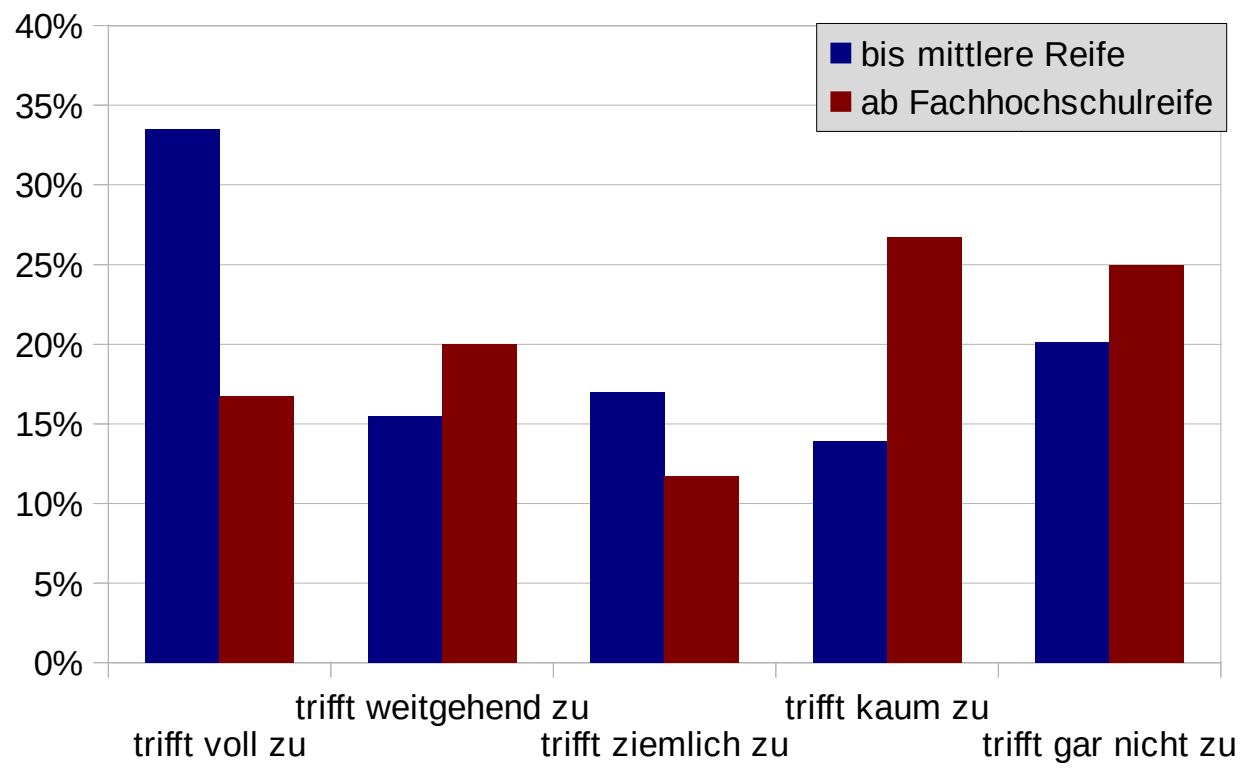

Abbildung 4.36: ...trinke ich weniger Alkohol (Auswertung nach Schulabschluss)

\subsubsection{Auswertung nach dem Familienstand}

Bei den Patienten mit Partner waren mit 63,8\% (n=123) Patienten bereit, ihren Alkoholkonsum teilweise oder ganz zu überdenken. Bei Patienten ohne Partner waren es demgegenüber 54,9\% $(n=34)$. Details finden sich in Tabelle 4.74. Der Unterschied war nicht signifikant $(p=0,463)$.

\begin{tabular}{|l|l|l|}
\hline & mit Partner & ohne Partner \\
\hline Trifft voll zu & $28,5 \%$ & $32,3 \%$ \\
\hline Trifft weitgehend zu & $18,7 \%$ & $9,7 \%$ \\
\hline Trifft ziemlich zu & $16,6 \%$ & $12,9 \%$ \\
\hline Trifft kaum zu & $17,1 \%$ & $17,7 \%$ \\
\hline Trifft gar nicht zu & $19,2 \%$ & $27,4 \%$ \\
\hline P-Wert & & \\
\hline
\end{tabular}

Tabelle 4.74: ...trinke ich weniger Alkohol (Auswertung nach Familienstand)

\subsubsection{Auswertung nach dem Alter}

Mit 57,0\% ( $n=49)$ bei den jüngeren und 62,9\% ( $n=108)$ bei den älteren beantworteten ähnlich viele Patienten in beiden Gruppen die Frage, ob sie nach Diagnosestellung weniger Alkohol getrunken hätten, mit „trifft voll zu“, „trifft weitgehend zu“ und „trifft ziemlich zu“ (Tabelle 4.75). Der Unterschied war nicht signifikant $(\mathrm{p}=0,100)$. 


\begin{tabular}{|c|c|c|}
\hline & Unter 65 & Über 66 \\
\hline Trifft voll zu & $22,1 \%$ & $32,6 \%$ \\
\hline Trifft weitgehend zu & $16,3 \%$ & $16,3 \%$ \\
\hline Trifft ziemlich zu & $18,6 \%$ & $14,0 \%$ \\
\hline Trifft kaum zu & $17,4 \%$ & $17,4 \%$ \\
\hline Trifft gar nicht zu & $25,6 \%$ & $19,8 \%$ \\
\hline P-Wert & & \\
\hline
\end{tabular}

Tabelle 4.75: ...trinke ich weniger Alkohol (Auswertung nach Alter)

\subsubsection{Beruf}

18,0\% ( $n=46)$ der Befragten übten/üben einen Beruf aus, der vornehmlich „draußen“, also ggf. unter Sonneneinstrahlung stattfindet/stattfand. 7 Patienten ließen die Frage unbeantwortet. Von den insgesamt 46 Befragten, die einen solchen Beruf ausübten, hatten 52,2\% ( $n=24)$ ihren Beruf nach Bekanntwerden ihrer Diagnose gewechselt. Dementsprechend behielten 47,8\% ( $\mathrm{n}=22)$ ihren Beruf bei.

\subsubsection{Geschlechtsspezifische Auswertung}

Mit 25,5\% ( $n=40)$ übten deutlich mehr Männer als Frauen (6,1\%; n=6) einen solchen Beruf aus. Zahlen zu der Anzahl der Patienten beiderlei Geschlechts, die ihren Beruf (mit vermehrter Sonnenexposition) wechselten, finden sich in Tabelle 4.76 .

\begin{tabular}{|l|l|lr|}
\hline & m & W & \\
\hline Beruf gewechselt & $47,5 \%$ & $83,3 \%$ & 0,190 \\
\hline Beruf nicht gewechselt & $52,5 \%$ & $16,7 \%$ & \\
\hline P-Wert & & &
\end{tabular}

Tabelle 4.76: Berufswechsel (geschlechtsspezifische Auswertung)

\subsubsection{Auswertung nach der Grunddiagnose}

26,8\% ( $n=11)$ der Patienten mit beiden Tumorentitäten, 19,1\% ( $n=26)$ mit nicht melanozytärem Tumor und 11,4\% (n=9) mit Melanom hatten einen Beruf mit vermehrter Sonnenexposition. Zahlen zu der Anzahl der Patienten (je nach Grunddiagnose), die ihren Beruf (mit vermehrter Sonnenexposition) wechselten, finden sich in Tabelle 4.77. 


\begin{tabular}{|l|l|l|l|}
\hline & Melanom & $\begin{array}{l}\text { Nicht-melanozytäre } \\
\text { Tumoren }\end{array}$ & $\begin{array}{l}\text { Melanom und } \\
\text { Nicht-melanozytäre } \\
\text { Tumoren }\end{array}$ \\
\hline Beruf gewechselt & $33,3 \%$ & $61,5 \%$ & $45,5 \%$ \\
\hline Beruf nicht gewechselt & $66,7 \%$ & $38,5 \%$ & $54,5 \%$ \\
\hline P-Wert & \multicolumn{2}{|l}{0,302} \\
\hline
\end{tabular}

Tabelle 4.77: Berufswechsel (Auswertung nach der Grunddiagnose)

\subsubsection{Auswertung nach dem Schulabschluss}

17,7\% ( $\mathrm{n}=34)$ der Patienten mit niedrigerem Schulabschluss übten einen Beruf aus, der mit vermehrter Sonnenexposition einherging. Bei den Patienten mit höherem Schulabschluss waren es 16,7\% ( $\mathrm{n}=10$ ). Zahlen zu der Anzahl der Patienten (je nach Schulabschluss), die ihren Beruf (mit vermehrter Sonnenexposition) wechselten, finden sich in Tabelle 4.78.

\begin{tabular}{|l|l|l|}
\hline & bis einschließlich mittlere Reife & ab Fachhochschulreife \\
\hline Beruf gewechselt & $47,1 \%$ & $60,0 \%$ \\
\hline Beruf nicht gewechselt & $52,9 \%$ & $40,0 \%$ \\
\hline P-Wert & \multicolumn{2}{|l|}{0,721} \\
\hline
\end{tabular}

Tabelle 4.78: Berufswechsel (Auswertung nach Schulabschluss)

\subsubsection{Auswertung nach dem Familienstand}

21,0\% ( $n=13)$ der Patienten ohne und 16,8\% ( $n=32)$ der Patienten mit Partner hatten einen Beruf mit vermehrter Sonnenexposition. Zahlen zu der Anzahl der Patienten (je nach Familienstand), die einen Beruf mit vermehrter Sonnenexposition ausgeübt hatten, und diesen wechselten, finden sich in Tabelle 4.79.

\begin{tabular}{|l|l|l|}
\hline & mit Partner & ohne Partner \\
\hline Beruf gewechselt & $53,1 \%$ & $46,2 \%$ \\
\hline Beruf nicht gewechselt & $46,9 \%$ & $53,8 \%$ \\
\hline P-Wert & & \\
\hline
\end{tabular}

Tabelle 4.79: Berufswechsel (Auswertung nach Familienstand)

\subsubsection{Auswertung nach dem Alter}

Unter den jüngeren Patienten (bis 65) übten 14,9\% ( $n=13$ ), unter den Älteren (über 66) 19,5\% $(n=33)$ einen Beruf mit erhöhter Sonnenexposition aus. Zahlen zu der Anzahl der Patienten (je nach 
Alter), die einen Beruf mit vermehrter Sonnenexposition ausgeübt hatten, und diesen wechselten, finden sich in Tabelle 4.80 .

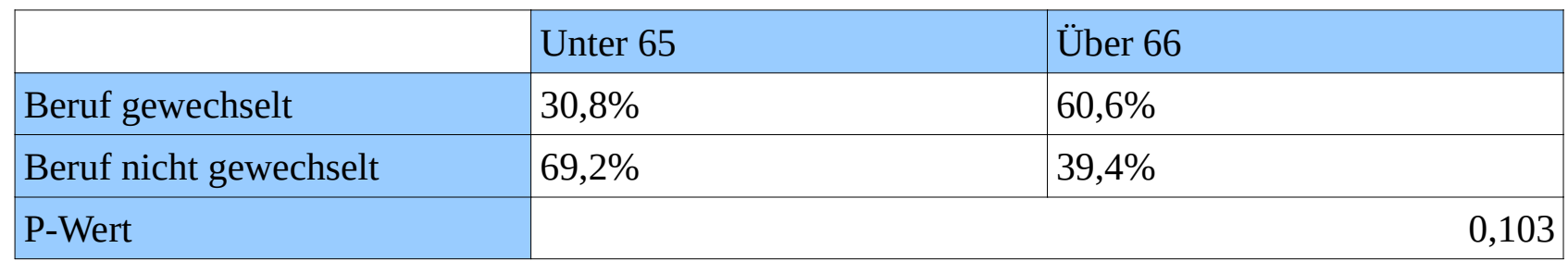

Tabelle 4.80: Berufswechsel (Auswertung nach Alter)

\subsubsection{Wahrnehmung der Hautkrebsvorsorgeuntersuchungen}

Die Patienten wurden befragt, inwiefern sie Hautkrebsvorsorgeuntersuchungen vor und nach ihrer Diagnose wahrgenommen hatten. Zudem wurde erfragt, wie oft sie diese Vorsorgeuntersuchung wahrgenommen hatten (einmal jährlich bzw. öfter). Gingen vor Bekanntwerden der Tumorerkrankung nur 34,9\% $(n=89)$ der Patienten zur Vorsorgeuntersuchung, waren es im Anschluss 89,1\% ( $\mathrm{n}=228$ ) der Befragten (Abbildung 4.37). Der Unterschied zeigte mit $\mathrm{p}<0,001$ eine statistische Signifikanz.

Auch kam es zu einer Zunahme hinsichtlich der Regelmäßigkeit der Wahrnehmung von Vorsorgeuntersuchungen. Es wurde erfragt, ob die Patienten, die die Vorsorgeuntersuchung wahrnahmen, mehr als einmal jährlich zur Vorsorgeuntersuchung gegangen waren. Von den 34,9\% ( $n=89$ ), die bereits vor der Diagnose einen Arzt aufgesucht hatten gingen 28,4\% ( $n=25)$ öfter als einmal jährlich zur Vorsorgeuntersuchung. Von den 89,1\% ( $\mathrm{n}=228)$, die nach der Diagnose ihres Tumors regelmäßig einen Arzt aufsuchten, gingen nun 77,5\% (n=172) öfter als einmal im Jahr zur Hautkrebsvorsorge (Abbildung 4.38). Auch dieser Unterschied ergab eine statistische Signifikanz $(\mathrm{p}<0,001)$. 


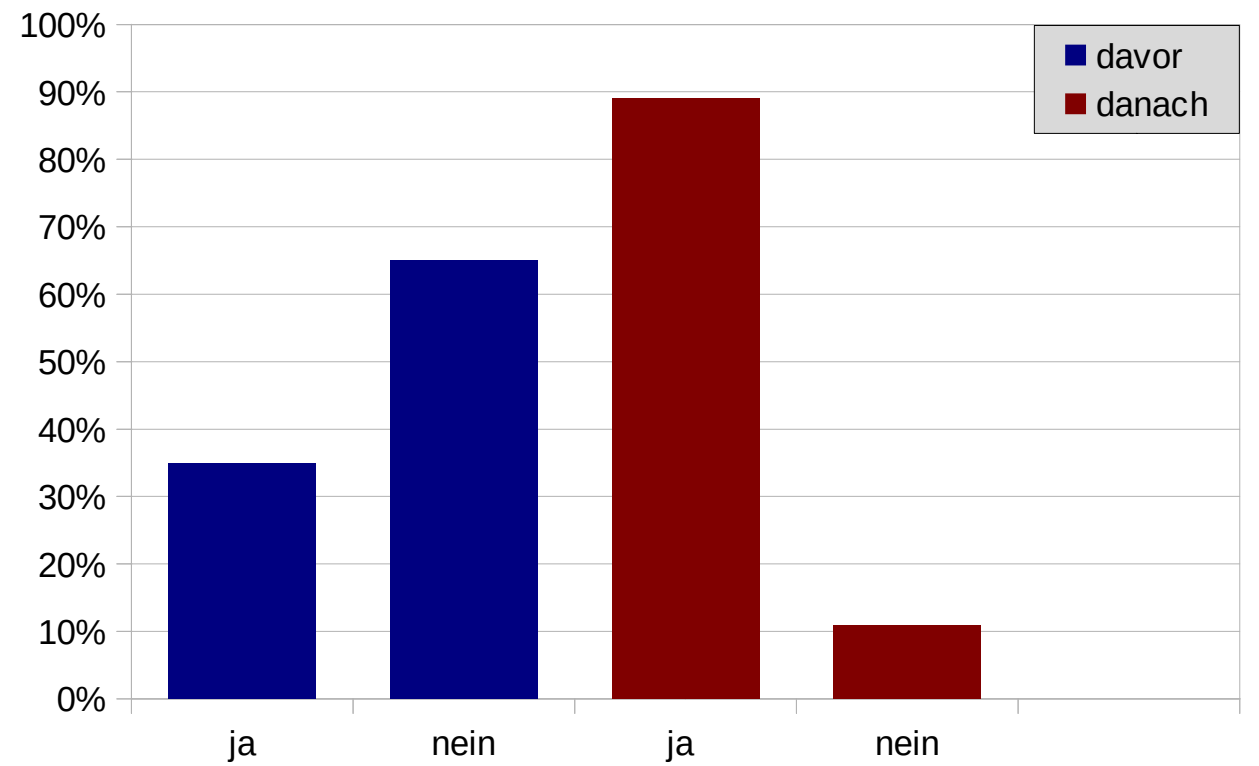

Abbildung 4.37: Sind Sie regelmäßig zur Hautkrebsvorsorgeuntersuchung gegangen?

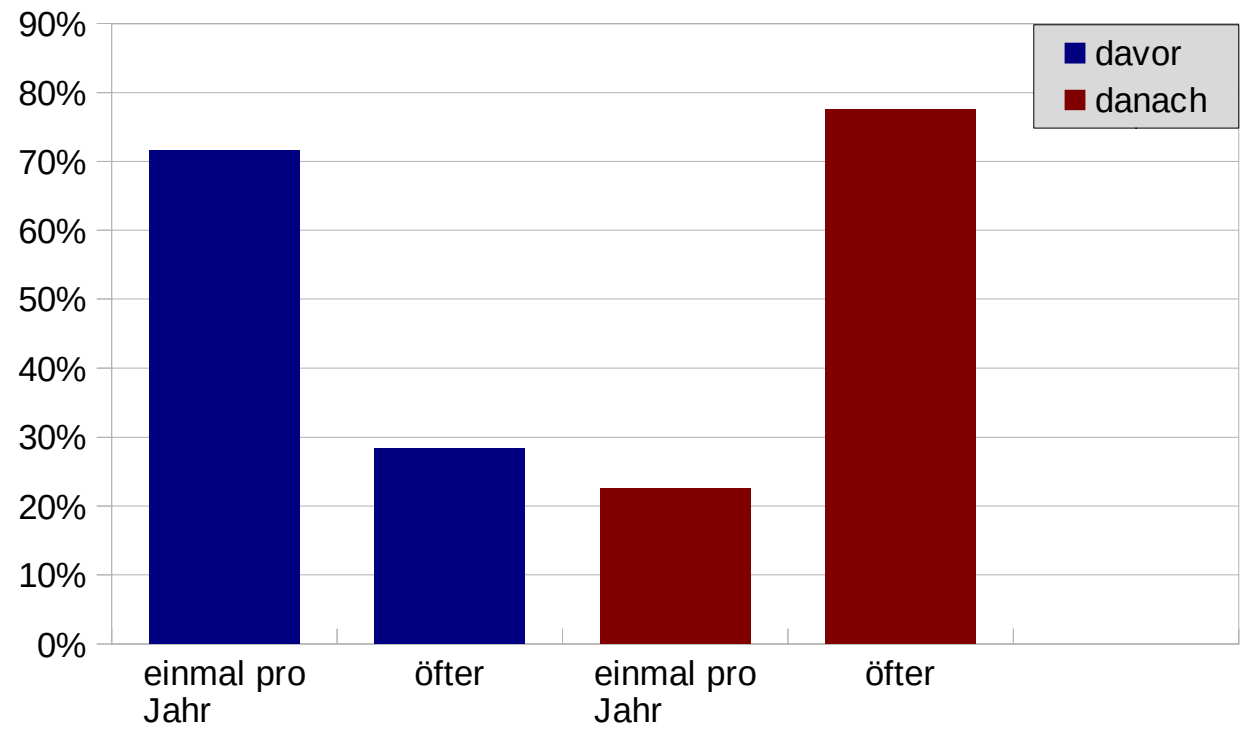

Abbildung 4.38: Frequenz der Vorsorgeuntersuchung

\subsubsection{Geschlechtsspezifische Unterschiede}

Bei Männern und Frauen zeigten sich ähnliche Verhaltensmuster. Bei den Männern gingen vor Bekanntwerden der Tumorerkrankung 32,5\% (n=51) regelmäßig zur Vorsorgeuntersuchung, danach 86,7\% ( $n=137)$. Bei den Frauen gingen davor 38,8\% $(n=38)$ regelmäßig zur Vorsorge, danach 92,9\% ( $\mathrm{n}=91)$. Sowohl davor, als auch danach waren es also vor allem die weiblichen Teilnehmer, die zur Vorsorge gingen. Der Unterschied zwischen Männern und Frauen zeigte sich weder vor 
$(\mathrm{p}=0,345)$ noch nach $(\mathrm{p}=0,151)$ der Diagnose statistisch signifikant (Abbildung 4.39).

Auf die Frage, ob die Vorsorgeuntersuchung einmal oder mehrfach jährlich wahrgenommen wurde, zeigte sich, dass vor der Diagnose, bei den Männern 32,0\% $(n=16)$ öfter als einmal im Jahr gingen, bei den Frauen hingegen nur 23,7\% (n=9). Danach waren es bei den Männern 74,4\% (n=99) und bei den Frauen 82,0\% ( $n=73)$. Der Unterschied zwischen Männern und Frauen war weder davor ( $\mathrm{p}=0,477)$ noch danach $(\mathrm{p}=0,195)$ statistisch signifikant (Abbildung 4.40).

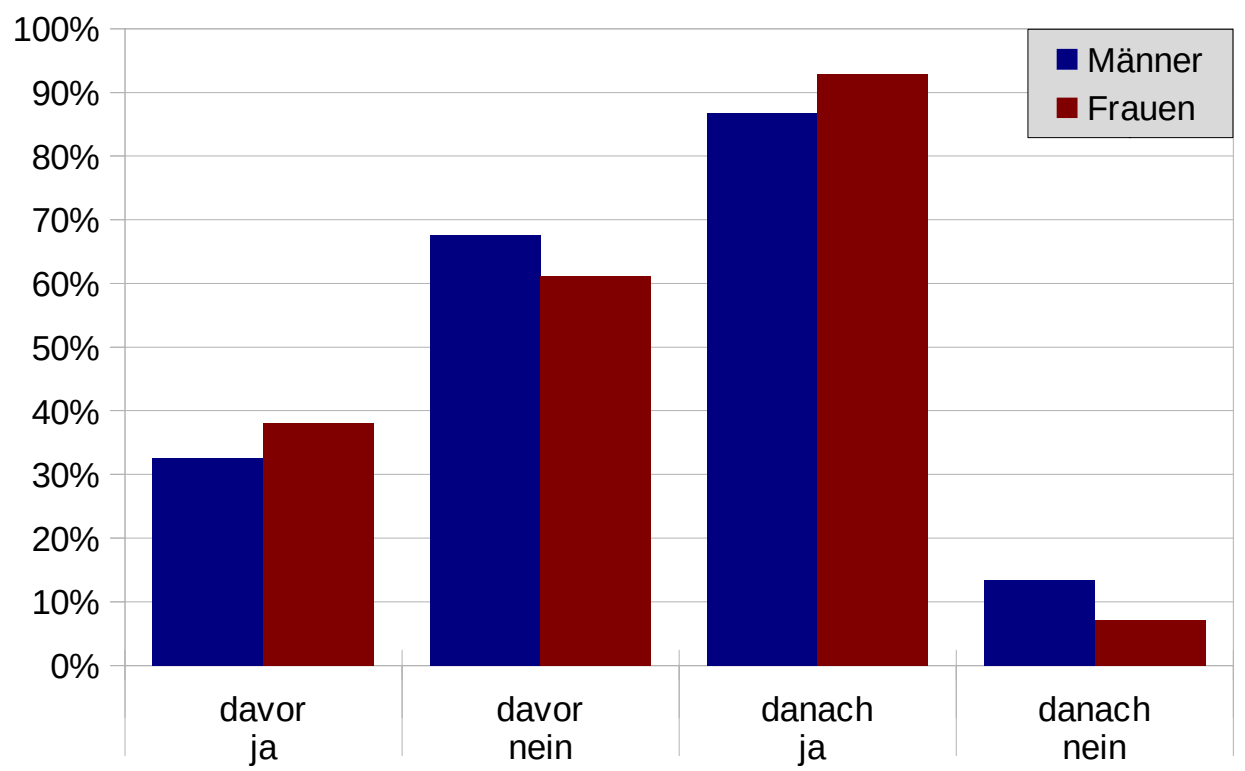

Abbildung 4.39: Sind Sie regelmäßig zur Hautkrebsvorsorgeuntersuchung gegangen? (geschlechtsspezifische Auswertung)

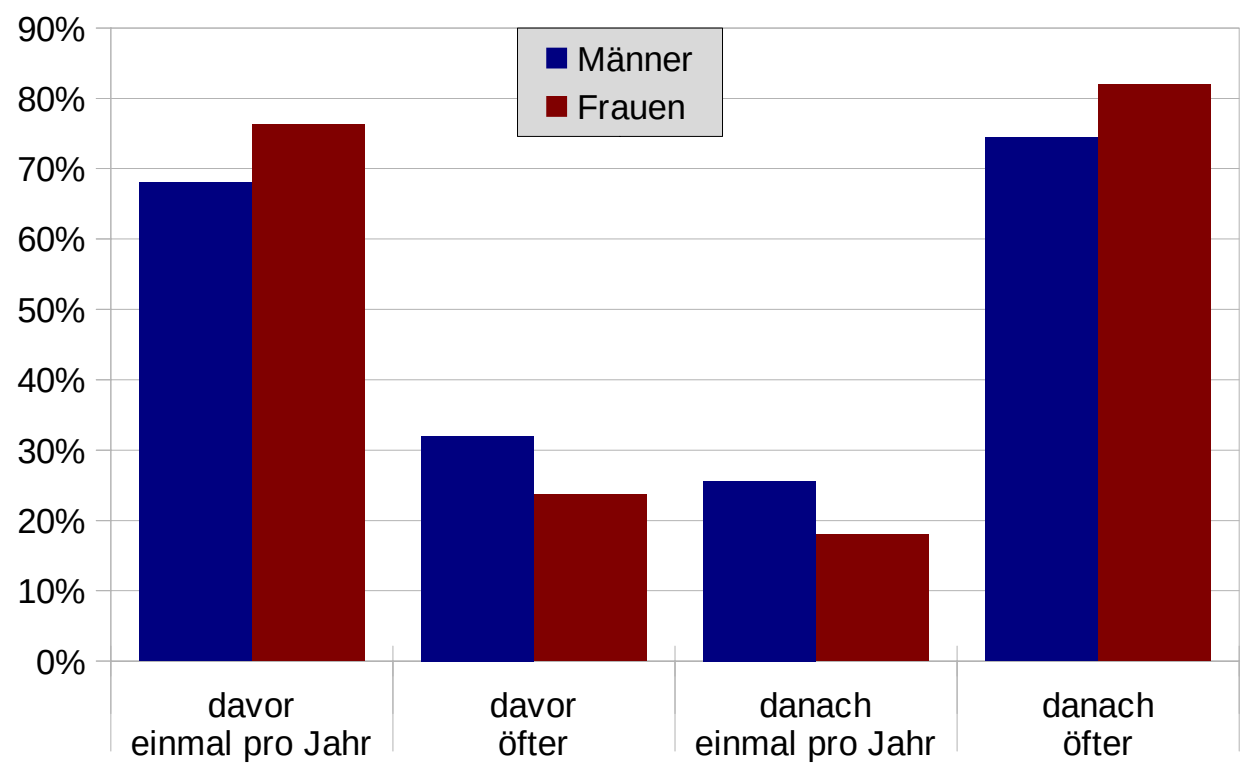

Abbildung 4.40: Frequenz der Vorsorgeuntersuchung (geschlechtsspezifische Auswertung) 


\subsubsection{Auswertung nach Grunddiagnose}

Auch hier zeigten sich in allen Gruppen ähnliche Verhaltensmuster. Bei Patienten, die unter beiden Tumorentitäten litten, gingen vor der Diagnose im Vergleich zu den anderen Gruppen mit 47,6\% $(n=20)$ bereits relativ viele zur Vorsorgeuntersuchung, danach waren es 92,5\% $(n=37)$. Bei den Melanompatienten waren es davor nur 31,2\% $(n=24)$, danach war es im Vergleich mit den anderen Gruppen mit 94,8\% (n=73) die größte Anzahl. Bei den Patienten mit nicht-melanozytärem Tumor waren es davor 33,1\% $(n=45)$ und danach 84,9\% $(n=118)$. Eine graphische Darstellung findet sich in Abbildung 4.41.

Die Unterschiede zwischen den verschiedenen Gruppen zeigten sich allerdings weder davor ( $\mathrm{p}=0,161)$ noch danach $(\mathrm{p}=0,062)$ signifikant.

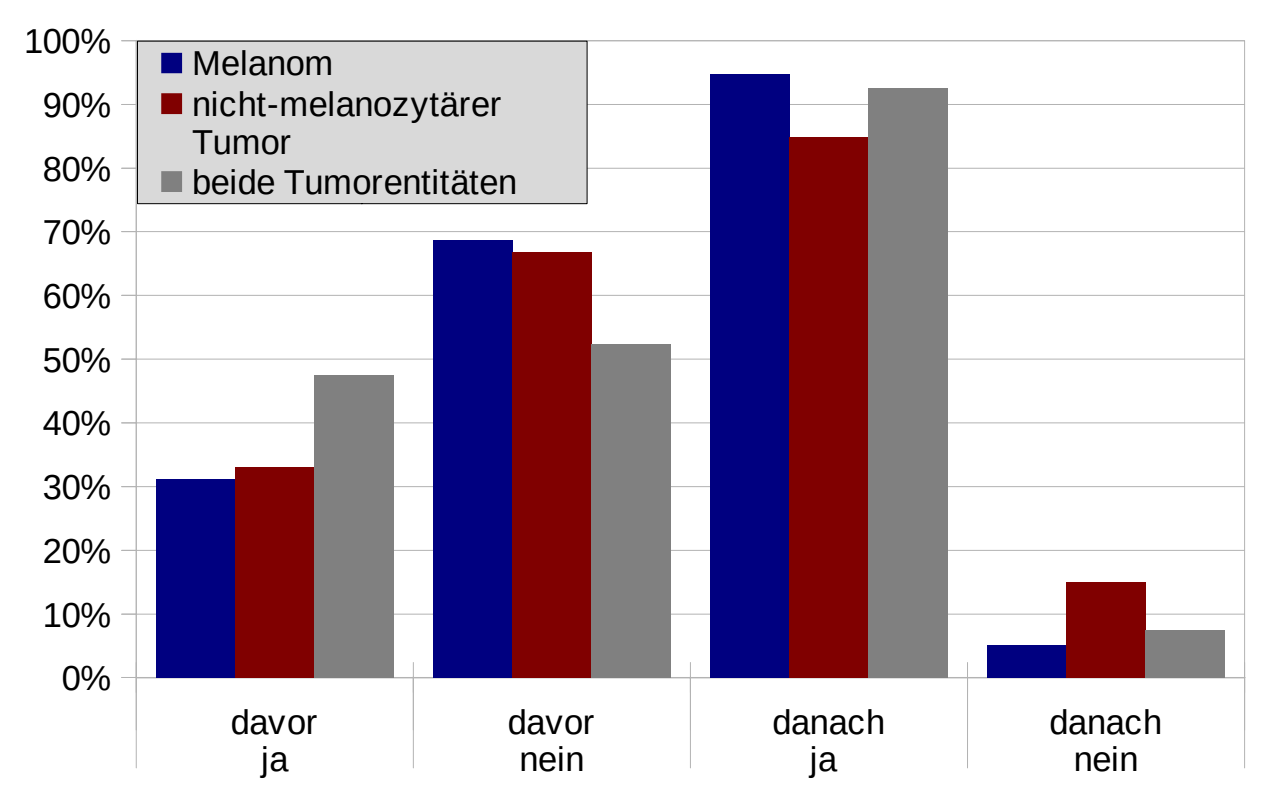

Abbildung 4.41: Sind Sie regelmäßig zur Hautkrebsvorsorgeuntersuchung gegangen? (Auswertung nach Grunddiagnose)

Auf die Frage, ob die Vorsorgeuntersuchung einmal oder mehrmals jährlich wahrgenommen wurde, zeigte sich, dass vor der Diagnose 33,3\% ( $n=8)$ der Melanompatienten, und 31,8\% (n=14) der Patienten mit nicht-melanozytären Tumoren öfter als einmal jährlich zur Vorsorge gegangen waren. Bei den Patienten mit beiden Tumorentitäten waren es nur 15,0\% (n=3). Der Unterschied war nicht statistisch signifikant $(\mathrm{p}=0,316)$.

Nach Bekanntwerden der Diagnose waren es vor allem die Melanompatienten (95,8\%; $n=69$ ) sowie Patienten mit beiden Tumorentitäten (86,1\%; $n=31)$, die öfter als einmal jährlich zur Vorsorge 
gingen. Bei Patienten mit nicht-melanozytärem Tumor waren es nur 63,2\% ( $n=72)$, welche die Vorsorge häufiger als einmal jährlich in Anspruch nahmen (Abbildung 4.42). Dieser Unterschied war statistisch signifikant $(\mathrm{p}<0,001)$.

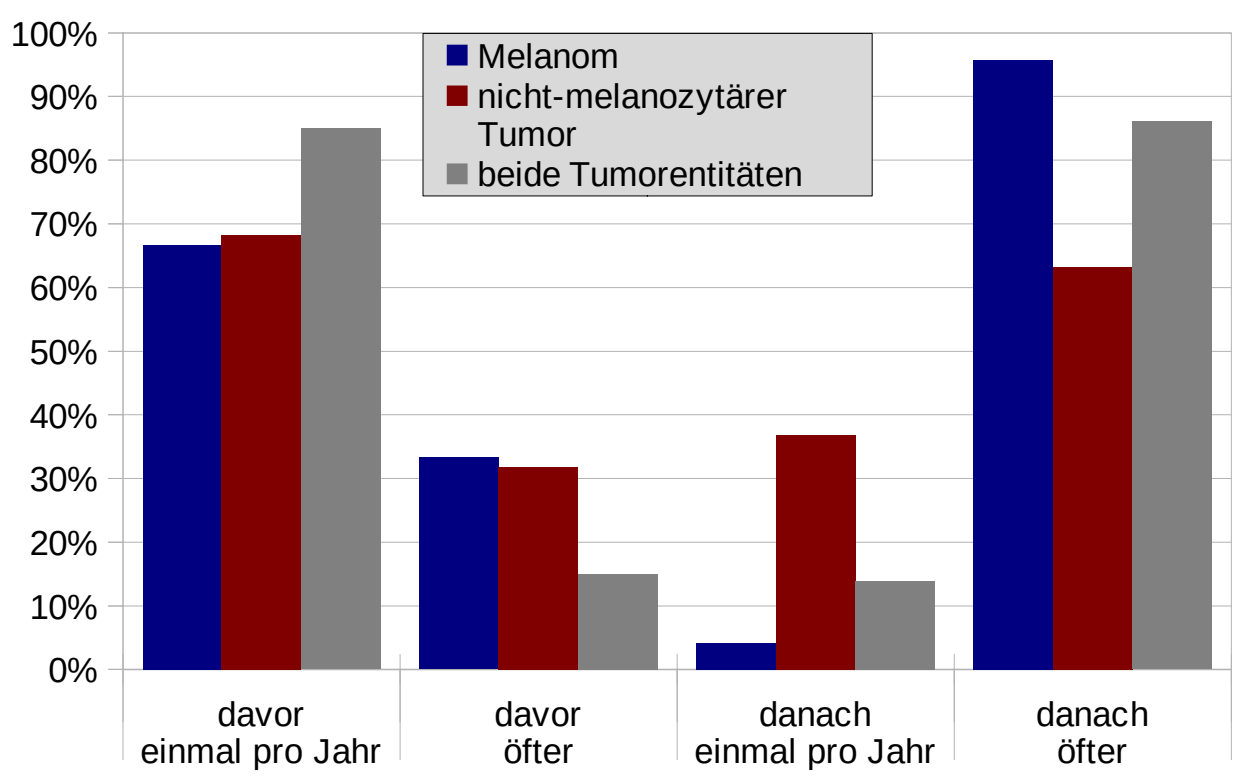

Abbildung 4.42: Frequenz der Vorsorgeuntersuchung (Auswertung nach der Grunddiagnose)

\subsubsection{Auswertung nach dem Schulabschluss}

Sowohl davor (geringere Qualifikation - 34,4\% ( $\mathrm{n}=66)$ vs. höhere Qualifikation - 35,6\% ( $\mathrm{n}=21$ ); $\mathrm{p}=0$,877), als auch danach (geringere Qualifikation 89,1\% ( $\mathrm{n}=172)$ vs. höhere Qualifikation 88,1\% $(n=52) ; p=0,815)$ ergaben sich keine signifikanten Unterschiede zwischen den Befragten, aufgeteilt nach ihrem Qualifikationsgrad (Abbildung 4.43).

Allerdings gingen vor Diagnosestellung die Befragten mit niedrigerem Qualifikationsgrad (34,8\%; $\mathrm{n}=23$ ) häufiger als einmal im Jahr zur Vorsorgeuntersuchung, als die Patienten mit höherer Qualifikation (10\%; $n=2)$. Das Ergebnis war signifikant ( $p=0,047)$.

Nach der Diagnose zeigte sich bei beiden Gruppen ein deutlicher Anstieg in der Frequenz des Wahrnehmens von Vorsorgeuntersuchungen. Bei Patienten mit niedrigerem Qualifikationsstand gingen 79,6\% ( $n=133)$ mehr als einmal pro Jahr zur Vorsorgeuntersuchung, bei denen mit höherem Qualifikationsstand waren es 73,1\% ( $\mathrm{n}=38)$. Der Unterschied war nicht signifikant $(\mathrm{p}=0,340)$. Eine Übersicht findet sich Abbildung 4.44. 


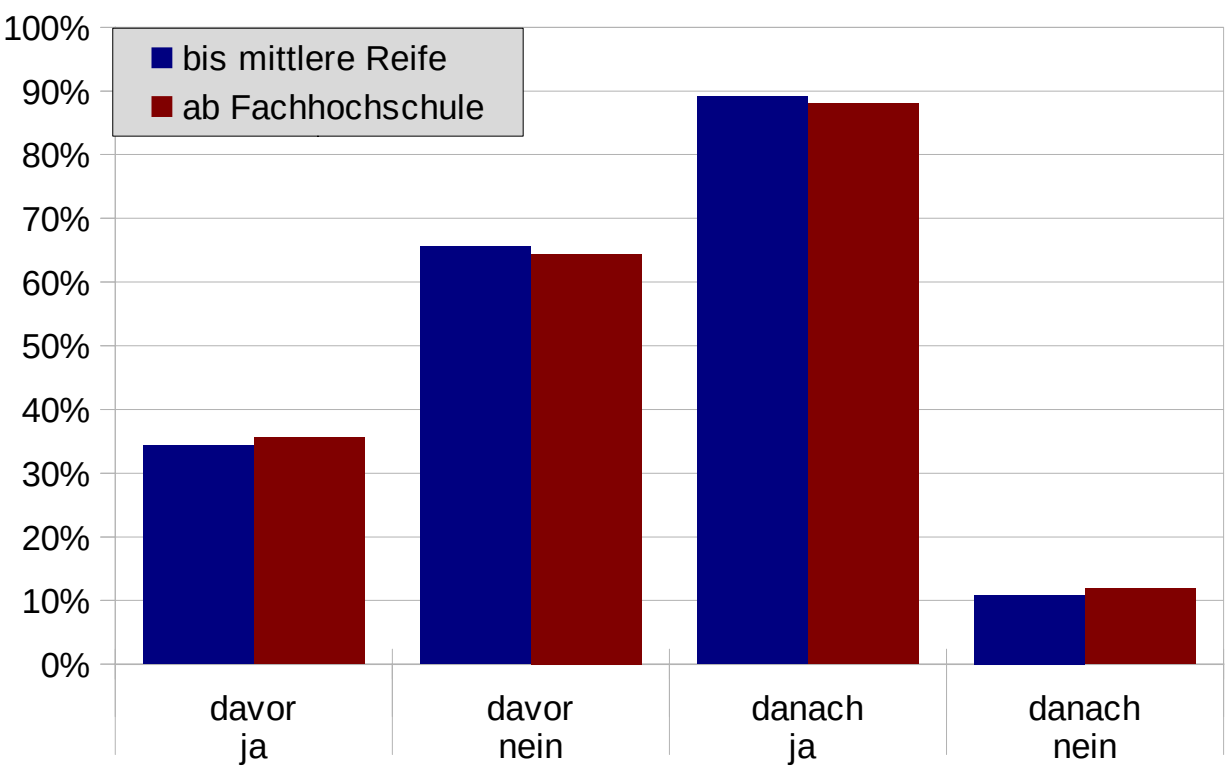

Abbildung 4.43: Sind Sie regelmäßig zur Hautkrebsvorsorgeuntersuchung gegangen? (Auswertung nach Schulabschluss)

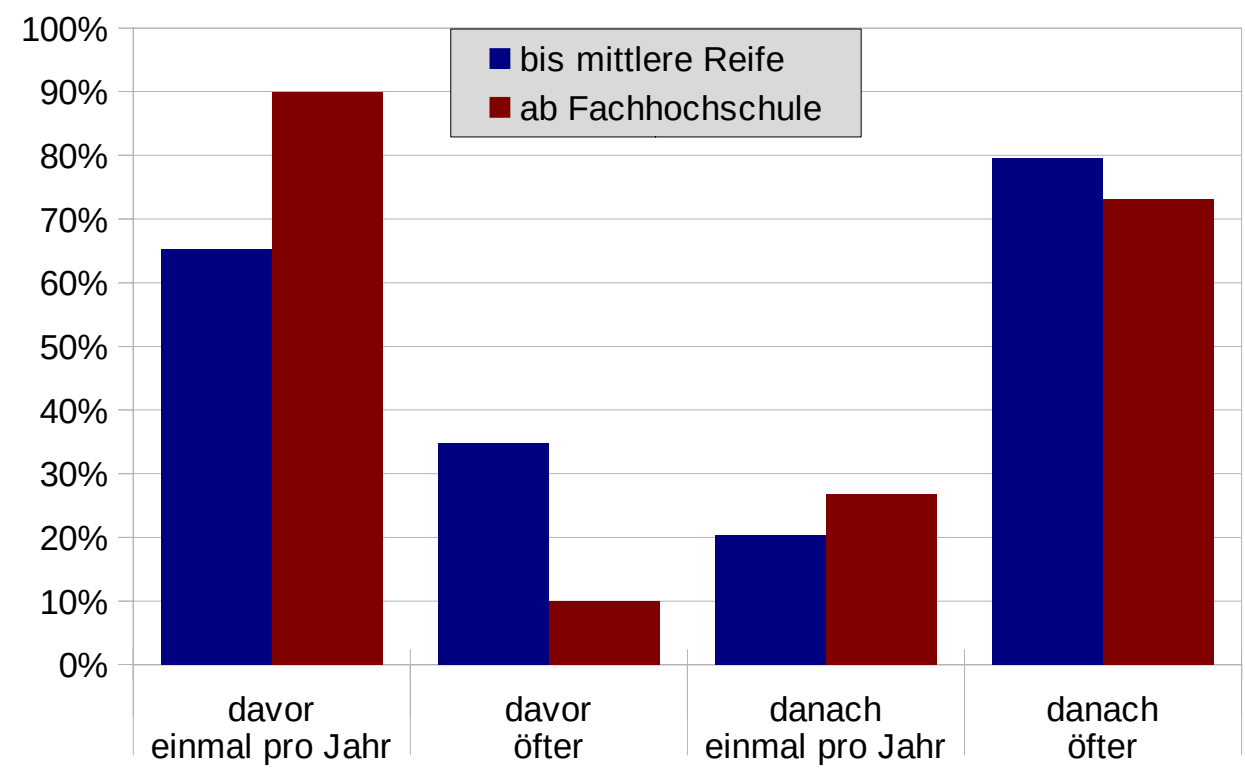

Abbildung 4.44: Frequenz der Vorsorgeuntersuchung (Auswertung nach Schulabschluss)

\subsubsection{Auswertung nach dem Familienstand}

Bei der Aufteilung der Patienten nach Familienstand zeigten sich folgende Ergebnisse. In beiden Gruppen (mit und ohne Partner lebend) gingen ähnlich viele Patienten vor der Diagnose zur Hautkrebsvorsorgeuntersuchung. Bei Patienten mit Partner waren es 33,5\% ( $n=64)$, bei denen ohne Partner 37,7\% ( $n=23)$. Nach der Diagnose waren es deutlich mehr Patienten, welche die 
Vorsorgeuntersuchung wahrnahmen. Bei den Patienten mit Partner waren es mit 90,5\% ( $\mathrm{n}=171$ ) etwas mehr als bei Patienten ohne $(84,4 \%$; $n=54)$.

Die Unterschiede zwischen beiden Gruppen zeigten sich allerdings weder davor $(p=0,541)$ noch danach $(p=0,247)$ signifikant. Eine graphische Darstellung findet sich in Abbildung 4.45.

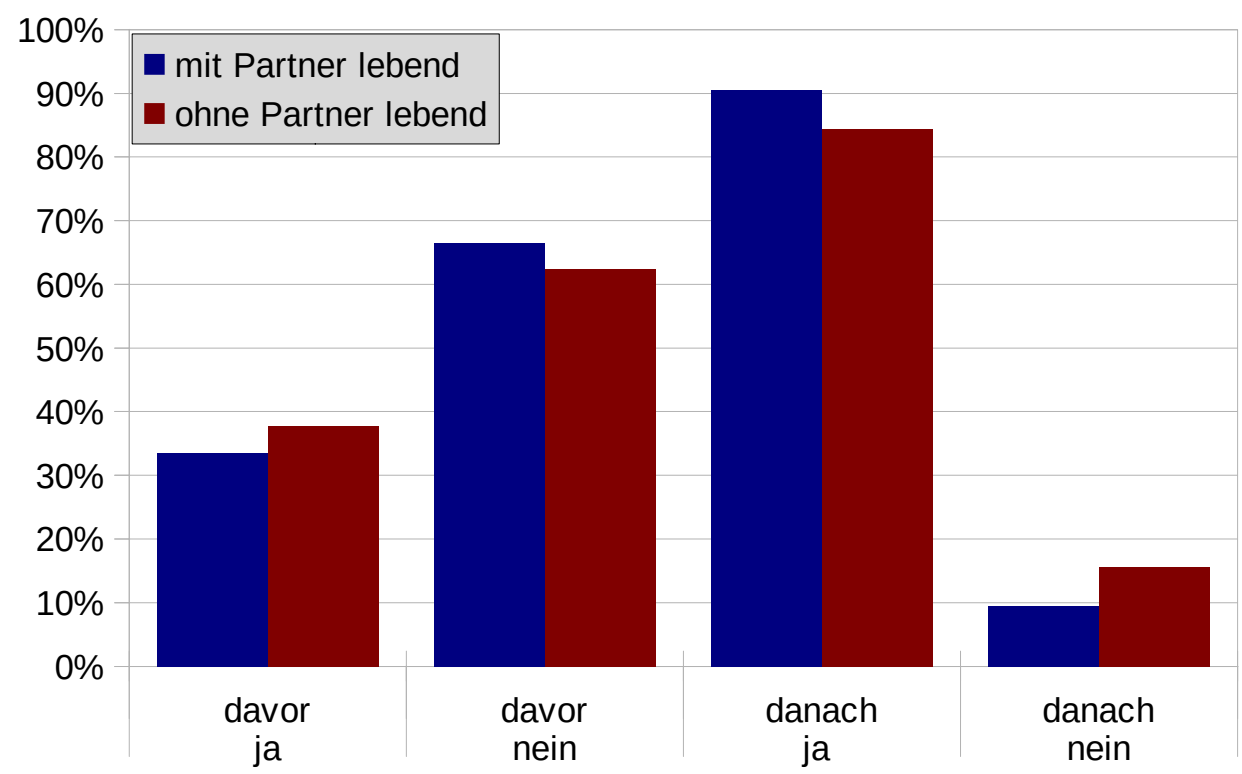

Abbildung 4.45: Sind Sie regelmäßig zur Hautkrebsvorsorgeuntersuchung gegangen? (Auswertung nach Familienstand)

Hinsichtlich der Wahrnehmung von Vorsorgeuntersuchungen vor Bekanntwerden der Diagnose (ein- bzw. mehrmals jährlich) zeigte sich folgendes Verhalten: Unter Patienten, die mit Partner lebten, waren 27\% ( $n=17)$, ohne Partner lebend 34,8\% $(n=8)$ öfter als einmal jährlich zur Vorsorgeuntersuchung gegangen (dieser Unterschied war statistisch nicht signifikant $-\mathrm{p}=0,593$ ). Nach Bekanntwerden der Diagnose waren es 78,7\% $(n=133)$ bei den Patienten mit Partner, die öfter als einmal jährlich zur Vorsorge gingen. Unter den Patienten ohne Partner waren es 74,5\% ( $\mathrm{n}=38$ ). Der Unterschied war statistisch nicht signifikant $(p=0,566)$. Eine graphische Darstellung findet sich in Abbildung 4.46. 


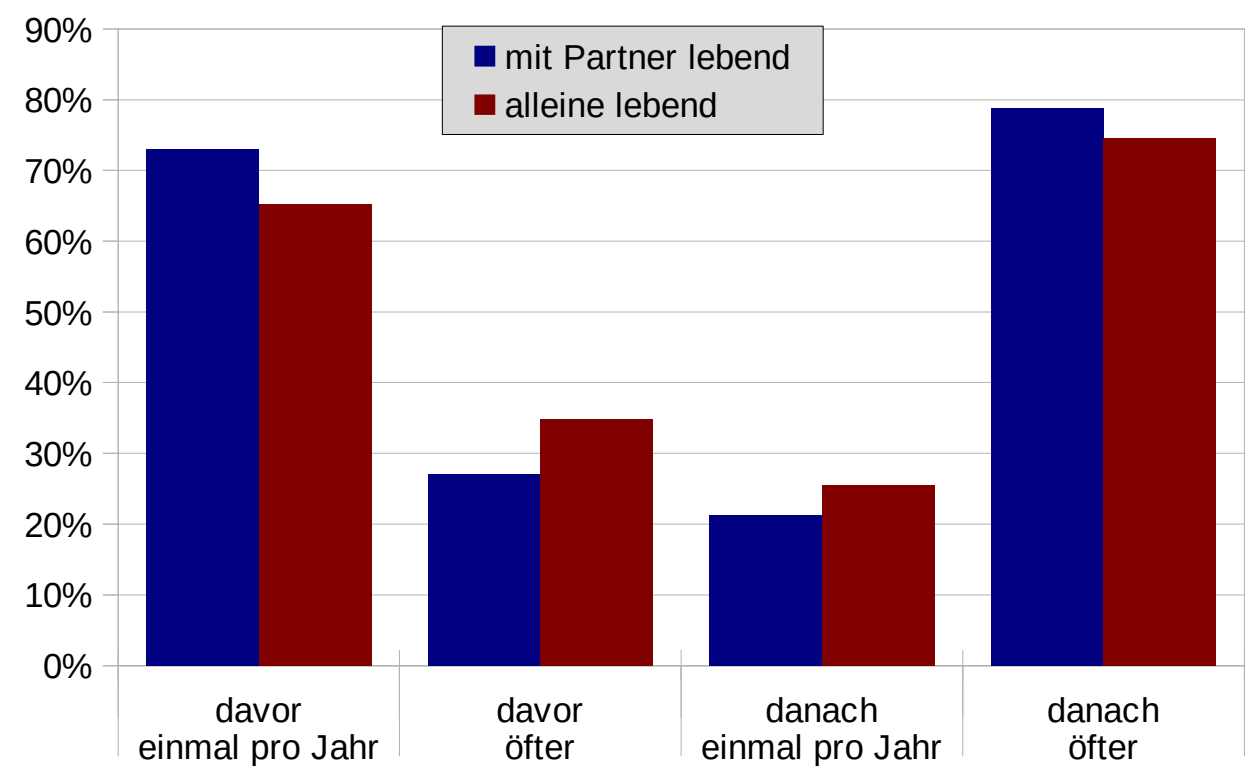

Abbildung 4.46: Frequenz der Vorsorgeuntersuchung (Auswertung nach Familienstand)

\subsubsection{Auswertung nach dem Alter}

Die unter 65-jährigen gingen vor der Diagnosestellung mit 33,3\% ( $n=29)$ etwas seltener zur Vorsorgeuntersuchung als ältere Patienten über 66 (35,7\%; $n=60)$. Der Unterschied war nicht signifikant $(\mathrm{p}=0,782)$. Nach Diagnosestellung waren es vor allem die jüngeren Patienten (97,6\%; $n=83$ ) die zur Hautkrebsvorsorge gingen. Bei den älteren Patienten waren es nur 84,8\% ( $n=145)$. Dieser Unterschied wies mit p=0,001 eine statistische Signifikanz auf (Abbildung 4.47).

In Bezug auf Vorsorgeuntersuchungen vor Bekanntwerden der Diagnose (ein- bzw. mehrmals jährlich) waren Patienten unter 65 Jahren zu 24,7\% (n=7), über 66 zu 30,5\% (n=18) öfter als einmal jährlich zur Vorsorge gegangen. Der Unterschied war statistisch insignifikant $(p=0,620)$.

Nach Bekanntwerden der Diagnose stiegen die Zahlen auf 80,0\% ( $n=64)$ unter den jüngeren bzw. 76,1\% ( $\mathrm{n}=108)$ unter den älteren Patienten. Der Unterschied war statistisch auch hier nicht signifikant $(\mathrm{p}=0,616)$. Eine graphische Darstellung findet sich in Abbildung 4.48. 


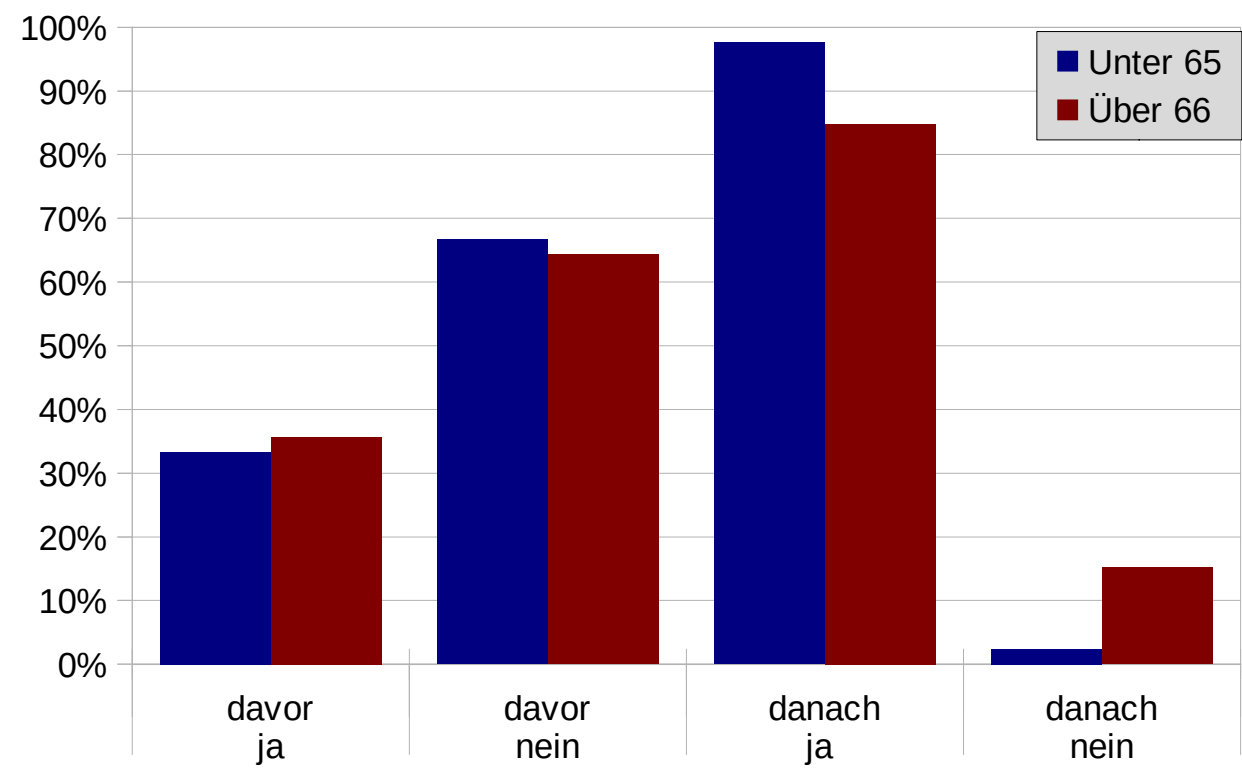

Abbildung 4.47: Sind Sie regelmäßig zur Hautkrebsvorsorgeuntersuchung gegangen? (Auswertung nach Alter)

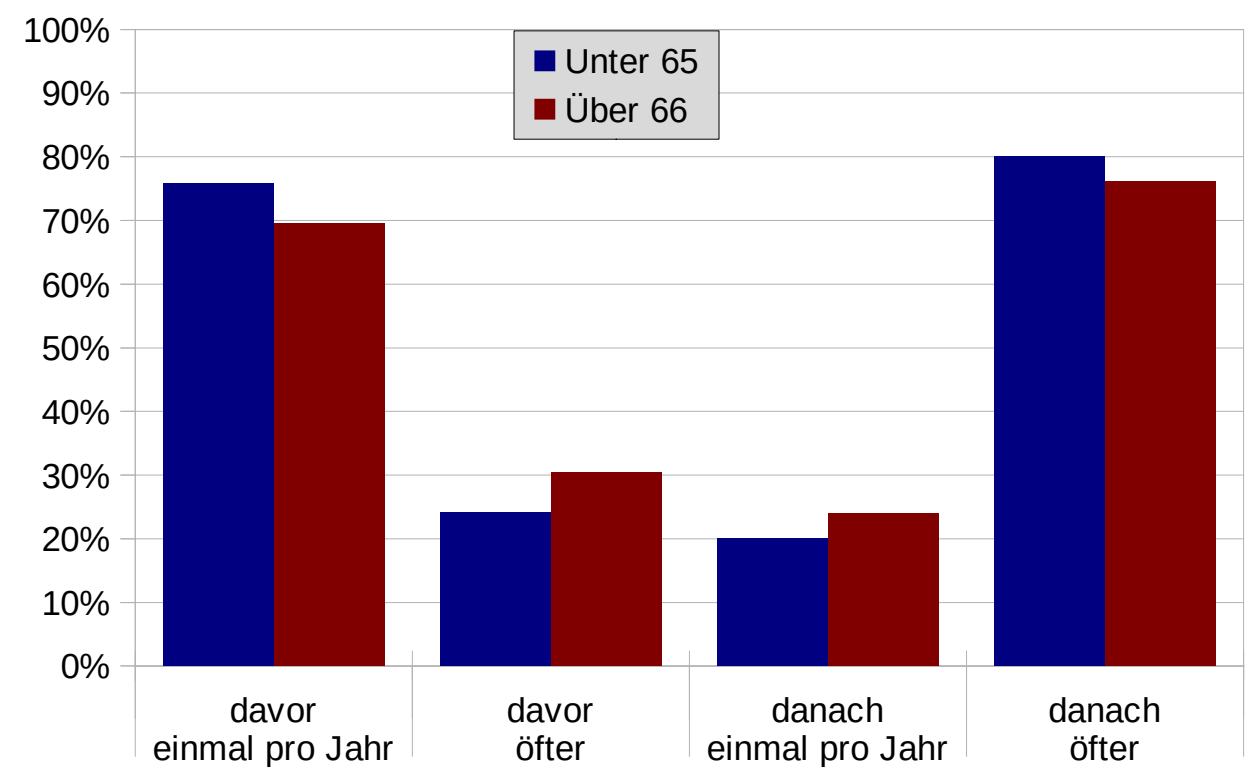

Abbildung 4.48: Frequenz der Vorsorgeuntersuchung (Auswertung nach dem Alter)

\subsubsection{Nikotinkonsum}

Unter den Befragten waren 14,8\% ( $\mathrm{n}=38)$ Raucher und 85,2\% ( $\mathrm{n}=219)$ Nicht-Raucher, wobei 6 Patienten diese Frage unbeantwortet ließen.

Nach dem Bekanntwerden ihrer Hauttumorerkrankung hatten in der Gruppe der Raucher 21,1\% 
$(n=8)$ mit dem Rauchen aufgehört, 42,1\% $(n=16)$ hatten das Rauchen reduziert und 36,8\% ( $n=14)$ hatten weiter geraucht (siehe Abbildung 4.49).

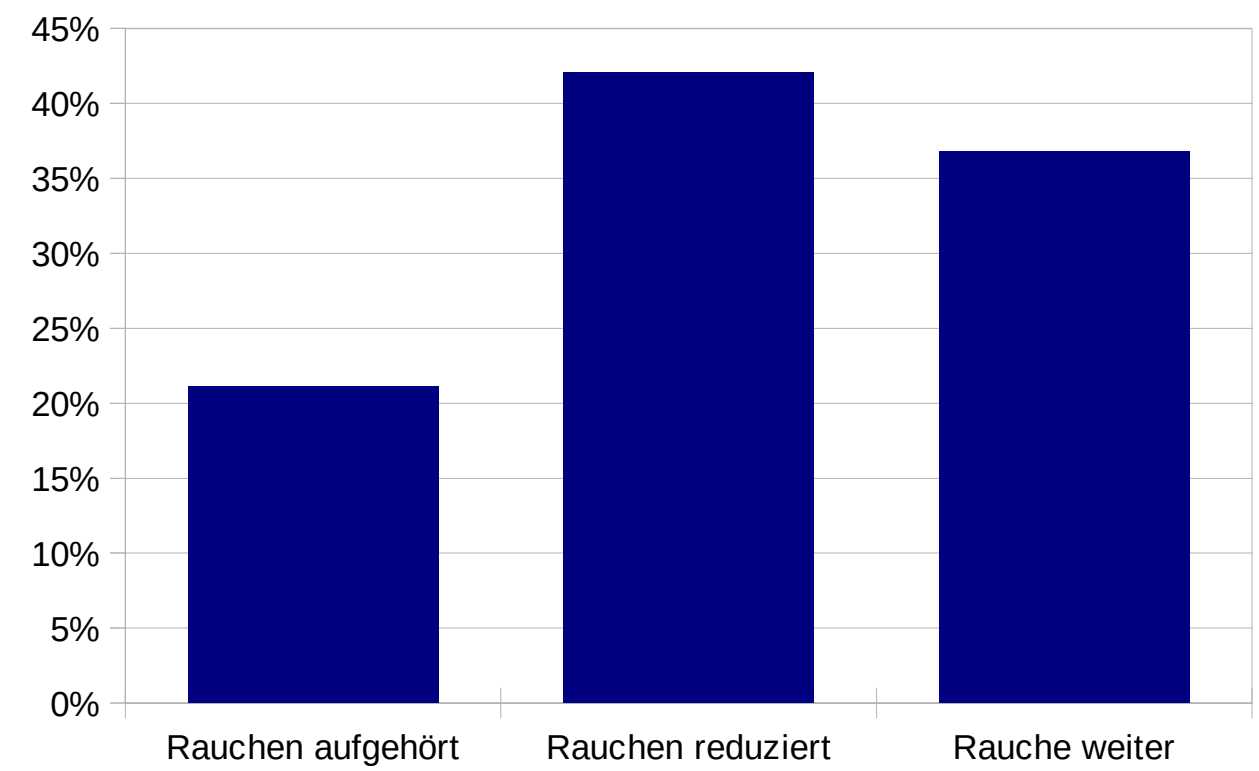

Abbildung 4.49: Nikotinkonsum nach Diagnose

\subsubsection{Geschlechtsspezifische Auswertung}

Insgesamt rauchten mehr Frauen (23,7\%; n=23) als Männer (9,4\%; n=15). Details, inwiefern der Nikotinkonsum durch die Diagnose beeinflusst wurde, sind Tabelle 4.81 zu entnehmen.

\begin{tabular}{|l|l|l|}
\hline & rauchende Männer & rauchende Frauen \\
\hline Rauchen aufgehört & $33,3 \%$ & $13,0 \%$ \\
\hline Rauchen reduziert & $40,0 \%$ & $43,5 \%$ \\
\hline Rauchen fortgesetzt & $26,7 \%$ & $43,5 \%$ \\
\hline P-Wert & \multicolumn{2}{|l|}{0,287} \\
\hline
\end{tabular}

Tabelle 4.81: Nikotinkonsum (geschlechtsspezifische Auswertung)

\subsubsection{Auswertung nach der Grunddiagnose}

19,5\% ( $n=15)$ der Melanompatienten, 12,9\% $(n=18)$ der Patienten mit nicht-melanozytären Tumor und 12,2\% $(\mathrm{n}=6)$ der Patienten mit beiden Tumoren waren Raucher. Details, inwiefern der Nikotinkonsum durch die Diagnose beeinflusst wurde, sind Tabelle 4.82 zu entnehmen. 


\begin{tabular}{|l|l|l|l|}
\hline & Raucher mit Melanom & $\begin{array}{l}\text { Raucher mit nicht- } \\
\text { melanozytärem Tumor }\end{array}$ & $\begin{array}{l}\text { Raucher mit Melanom und } \\
\text { nicht-melanozytärem } \\
\text { Tumor }\end{array}$ \\
\hline Rauchen aufgehört & $6,7 \%$ & $27,8 \%$ & $40,0 \%$ \\
\hline Rauchen reduziert & $40,0 \%$ & $44,4 \%$ & $40,0 \%$ \\
\hline Rauchen fortgesetzt & $53,3 \%$ & $27,8 \%$ & $20,0 \%$ \\
\hline P-Wert & \multicolumn{3}{|l}{0,325} \\
\hline
\end{tabular}

Tabelle 4.82: Nikotinkonsum (Auswertung nach Grunddiagnose)

\subsubsection{Auswertung nach dem Schulabschluss}

15,5\% ( $n=30)$ der Patienten mit niedrigerem und 13,3\% $(n=8)$ der Patienten mit höherem Bildungsabschluss waren Raucher. Details, inwiefern der Nikotinkonsum durch die Diagnose beeinflusst wurde, sind Tabelle 4.83 zu entnehmen.

\begin{tabular}{|l|l|l|}
\hline & Raucher, bis mittlere Reife & Raucher, ab Fachhochschulreife \\
\hline Rauchen aufgehört & $23,3 \%$ & $12,5 \%$ \\
\hline Rauchen reduziert & $43,3 \%$ & $37,5 \%$ \\
\hline Rauchen fortgesetzt & $33,3 \%$ & $50,0 \%$ \\
\hline P-Wert & \multicolumn{2}{|l|}{0,644} \\
\hline
\end{tabular}

Tabelle 4.83: Nikotinkonsum (Auswertung nach Schulabschluss)

\subsubsection{Auswertung nach dem Familienstand}

Mit 20,6\% ( $\mathrm{n}=13)$ waren es vor allem Patienten ohne Partner, die rauchten. Bei mit Partner lebenden Patienten waren 13,0\% $(n=25)$ Raucher. Details, inwiefern der Nikotinkonsum durch die Diagnose beeinflusst wurde, sind Tabelle 4.84 zu entnehmen.

\begin{tabular}{|l|l|l|}
\hline & Raucher ohne Partner & Raucher mit Partner \\
\hline Rauchen aufgehört & $30,8 \%$ & $16,0 \%$ \\
\hline Rauchen reduziert & $38,5 \%$ & $44,0 \%$ \\
\hline Rauchen fortgesetzt & $30,8 \%$ & $40,0 \%$ \\
\hline P-Wert & & \\
\hline
\end{tabular}

Tabelle 4.84: Nikotinkonsum (Auswertung nach Familienstand) 


\subsubsection{Auswertung nach dem Alter}

Unter den jüngeren waren weitaus mehr Raucher als unter den älteren Patienten (36,5\%; n=31 bei

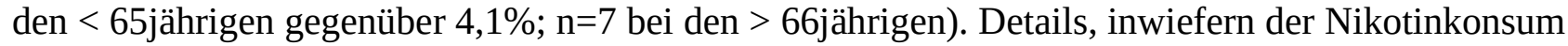
durch die Diagnose beeinflusst wurde, sind Tabelle 4.85 zu entnehmen.

\begin{tabular}{|l|l|l|}
\hline & Raucher unter 65 Jahre & Raucher über 66 Jahre \\
\hline Rauchen aufgehört & $16,1 \%$ & $42,9 \%$ \\
\hline Rauchen reduziert & $38,7 \%$ & $57,1 \%$ \\
\hline Rauchen fortgesetzt & $45,2 \%$ & $0,0 \%$ \\
\hline P-Wert & & \\
\hline
\end{tabular}

Tabelle 4.85: Nikotinkonsum (Auswertung nach Alter) 


\section{Diskussion}

\subsection{Allgemeine Limitationen}

Für unsere Studie wählten wir ein retrospektives Studiendesign. Diese Art des Designs ist ein hervorragendes Mittel zur raschen Erfassung und Auswertung einer großen Anzahl bereits bestehender Daten. Zudem lassen sich hiermit häufig Hypothesen entwickeln, die Ansatzpunkte für spätere prospektive Studien bieten können [76].

Allerdings gab es, wie üblich bei retrospektiven Studien, einige Limitationen, die HESS in seiner Arbeit erläuterte. Die Wichtigsten, insbesondere in Hinblick auf unsere Studie, sind unter den folgenden Stichpunkten aufgeführt [36].

- Wie auch im Falle des Homburger Gesundheitsbogens, fehlt bei retrospektiven Studien häufig die Kontrollgruppe (z.B. gesunde Gesamtpopulation oder dieselben Patienten vor Diagnosestellung), mit der die Daten verglichen werden können.

- Es werden Daten aus Patientenakten sowie aus den beantworteten Fragebögen erhoben. Daher sind die Ergebnisse von der Genauigkeit dieser Daten sehr abhängig. Es kann nicht immer davon ausgegangenen werden, dass alle Patientenakten in derselben Qualität geführt werden, oder dass sich die Patienten noch an die einzelnen Umstände erinnern, die ihnen erlaubten, den Fragebogen zu beantworten ('recall bias').

- Eine retrospektiven Studie kann nicht rückwirkend randomisiert oder verblindet werden.

- Da wir vor der Studie festgelegt hatten, nur Patienten zu berücksichtigen, die bereits an Hautkrebs erkrankt waren, fand im Vorfeld eine Selektion statt. Unsere Gruppe war somit nicht repräsentativ für die deutsche Allgemeinbevölkerung (`selection bias`).

Darüber hinaus fand eine Selektion durch die Patienten selbst statt. Beispielsweise untersuchten EYSENBACH und WYATT die Möglichkeit, Studien mit internetbasierten Fragebögen durchzuführen. Sie beschrieben diesbezüglich die Problematik eines sogenannten `volunteer effects '. Unter diesem Begriff versteht man, dass nur Menschen erfasst werden, die den Fragebogen auch ausfüllen und dazu bereit sind, teilzunehmen. Das ist die Selektion durch die Patienten selbst: es kommt zu einer Überrepräsentation von bestimmten Gruppen, die nicht die Durchschnittsbevölkerung abbilden [23].

Ein weiterer zu berücksichtigender Punkt ist, dass die von uns befragten Patienten auch durch uns 
behandelt wurden. Deshalb war es vorstellbar, dass die Befragten Antworten gaben, mit denen sie glaubten, auf unsere Zustimmung zu treffen (`social desirability bias’) [44].

\subsection{Patientenübersicht und häufigste Antworten}

Führt man die typischsten Merkmale aller Patienten der vorliegenden Studie zusammen, so war der häufigste Patient männlich, zwischen 66 und 85 Jahre alt, Nicht-Raucher und litt unter einem nichtmelanozytären Tumor. Er hatte als höchsten Abschluss einen Hauptschulabschluss und übte einen Beruf aus, der nicht mit vermehrter Sonnenexposition einherging. Er war verheiratet und zeigte sich in allen Domänen der Lebensqualität entsprechend des WHOQOL-BREF (Physisch, Psychisch, Soziale Beziehungen, Umwelt und Globalwert) zufriedener als die deutsche Allgemeinbevölkerung, wobei die Zufriedenheit mit der „Umwelt“ am ausgeprägtesten war. Er bewegte sich nicht mehr als vor Bekanntwerden seiner Hautkrebserkrankung und hatte auch keine neue Sportart begonnen. In Bezug auf die UV-Exposition achtete er darauf, sich weniger in der Sonne aufzuhalten, benutzte häufiger Sonnenschutzcremes und trug häufiger einen Sonnenhut. Außerdem achtete er darauf, das Haus in den Mittagsstunden nicht zu verlassen. Das Urlaubsverhalten wurde angepasst, es wurden keine Strandurlaube mehr unternommen. Das Freizeitverhalten (Radtouren etc.) hingegen wurde nicht angepasst. Der Alkoholkonsum wurde nach Bekanntwerden der Hauttumorerkrankung reduziert. Er ernährte sich gesünder und bewusster. Der häufigste Patient ging vor der Diagnose nicht zur Vorsorgeuntersuchung, danach hingegen öfter als einmal jährlich.

\subsection{Patientenstatistik}

Insgesamt waren 653 Patienten angeschrieben worden von denen 61,7\% männlich und 38,3\% weiblich waren. Die männlichen Patienten waren somit überrepräsentiert. Allerdings war die Rücklaufquote mit ca. $40 \%$ bei beiden Geschlechtern nahezu identisch, so dass es diesbezüglich kaum zu Verzerrungen kam.

Bei den Melanompatienten unserer Studie lag der Altersdurchschnitt bei 68,82, bei den Patientinnen bei 57,65 Jahren. Verglich man diese Werte mit denen des Robert-Koch-Instituts, ergaben sich keine großen Unterschiede zwischen dem Altersdurchschnitt unserer Patienten und dem mittleren Erkrankungsalter für das maligne Melanom in Deutschland (Mittleres Erkrankungsalter Männer 67 Jahre; Frauen 60 Jahre) [63].

Der Altersdurchschnitt der Patienten mit nicht-melanozytärem Tumor unserer Studie lag bei 75,7 Jahren bei den Männern und 69,5 Jahren bei den Frauen. Laut Robert-Koch-Institut lag das mittlere 
Erkrankungsalter für das Basaliom für Männer bei 73 und für Frauen bei 71 Jahren, beim Plattenepithelkarzinom für Männer bei 77 und für Frauen bei 79 Jahren [63]. Daher lag vor allem der Altersdurchschnitt der an unserer Studie teilnehmenden Frauen mit nicht-melanozytärem Tumor deutlich unter dem mittleren Erkrankungsalter für Basaliome und Plattenepithelkarzinome.

\subsubsection{Tumorentität}

Mit 53,6\% litten die meisten unserer Patienten unter einem nicht melanozytären Tumor. Darauf folgten mit 30\% die Patienten mit Melanom. 16,3\% litten unter beiden Tumorentitäten.

Der nicht-melanozytäre Hautkrebs ist in Deutschland 6,5mal häufiger als das Melanom [29]. Im Vergleich dieses Wertes mit unseren Ergebnissen zeigte sich, dass die Patienten mit Melanomen in unserer Studie deutlich überrepräsentiert waren. Am ehesten ist dies darauf zurückzuführen, dass die teilnehmenden Patienten sich bereits in der dermatologischen Abteilung des Universitätsklinikums des Saarlandes vorgestellt hatten. Es ist anzunehmen, dass viele dieser Patienten vorher beim niedergelassenen Dermatologen gewesen waren und bei entsprechender Schwere der Erkrankung, z.B. bei einem Melanom, eine Überweisung in die Universitätsklinik erhalten hatten. Diese Art der Verzerrung ('selection bias') wurde bereits unter 'Allgemeine Limitationen’ erwähnt [36].

\subsubsection{Qualifikation}

Unter den Befragten hatten 54,4\% einen Hauptschulabschluss als höchste Qualifikation; nur 23,5\% hatten einen höheren Abschluss als die Mittlere Reife.

Vergleicht man diese Werte mit denen des Statistischen Bundesamtes aus dem Jahre 2014, zeigt sich, dass hier 33,8\% der Bevölkerung einen Hauptschulabschluss, 22,7\% einen Realschulabschluss und 28,8\% eine (Fach-) Hochschulreife hatten [69].

Daraus geht hervor, dass der Bildungsstand unter den Teilnehmern in dieser Studie insgesamt niedriger als in der deutschen Allgemeinbevölkerung war.

Es ist zu bedenken, dass die Werte des Statistischen Bundesamtes nicht auf die hier vorliegenden Teilnehmer altersstandardisiert wurden. Der Altersdurchschnitt der Patienten unserer Studie liegt beträchtlich höher als der des Statistischen Bundesamtes. In der Tat geht aus dem Mikrozensus des Statistischen Bundesamtes des Jahres 2014 lt. Aufteilung nach Altersgruppen hervor, dass der Anteil der Hauptschüler bei den über 60 bis 65 jährigen bedeutend höher ist als bei jüngeren Gruppen [73]. 


\subsubsection{Familienstand}

Der Großteil der Patienten (67,3\%; $n=175)$ war verheiratet; mit 13,8\% (n=36) war ein relativ großer Teil verwitwet. Insgesamt lebten ca. 25\% der Patienten alleine (geschieden, ledig, verwitwet etc.) und ca. 75\% mit Partner (verheiratet oder in Partnerschaft). Vergleichsweise dazu sind unter der erwachsenen deutschen Bevölkerung 53\% verheiratet und ca. 26\% ledig [13]. Der große Anteil Verwitweter in dieser Studie ist aller Wahrscheinlichkeit nach auf das hohe Durchschnittsalter unserer Patienten zurückzuführen.

\subsection{WHOQOL-BREF}

Zum besseren Verständnis folgt eine kurze Erläuterung zu den Domänen des WHOQOL-BREF: Damit die Lebensqualität unserer Patienten mit den Werten der deutschen Allgemeinbevölkerung verglichen werden konnte, wurden die Antworten der insgesamt 26 Fragen 5 Domänen zugeordnet. So beinhaltete die Domäne „Physisch“ die Zufriedenheit in Bezug auf das körperliche Wohlbefinden (Schmerzen, Mobilität, Arbeitsfähigkeit, Bedarf von regelmäßiger medizinischer Behandlung, etc.). Die Domäne „Psychisch“ erfasste vor allem das psychische Wohl (Selbstbewusstsein, Zufriedenheit mit dem eigenen Körper, Glaubenseinstellung, etc.). In der Domäne „Soziale Beziehungen“ lag das Augenmerk v.a. auf der Zufriedenheit mit dem persönlichen Umfeld (Freunde, sexuelle Aktivität, etc.). Mit der Domäne „Umwelt“ wurde die Zufriedenheit mit der persönlichen Umgebung (häusliches Umfeld, finanzielle Rücklagen, das Angebot an Freizeitaktivitäten, Mobilität, Sicherheitsgefühl, etc.) erfasst. Mit dem „Globalwert“ wurden der „Allgemeine Gesundheitszustand“ bzw. die „Zufriedenheit mit dem Gesundheitszustand“ beschrieben [2].

Mit unserer Studie sollte geprüft werden, ob eine Erkrankung an Hautkrebs Einfluss auf die Lebensqualität hatte und sich somit Ansatzpunkte boten, die Compliance der Patienten zu verbessern. Entgegen unserer Erwartungen waren die von uns untersuchten Patienten in jeder der durch den WHOQOL-BREF geprüften fünf Dömanen zufriedener als die altersstandardisierte deutsche Allgemeinbevölkerung. Besonders zufrieden waren unsere Patienten mit ihrer psychischen Situation, ihrer Umwelt und ihren sozialen Beziehungen. Diesbezüglich ergab sich im Vergleich zur deutschen Allgemeinbevölkerung auch eine statistische Signifikanz. Die Zufriedenheit unserer Patienten mit ihrem allgemeinen Gesundheitszustand („Globalwert“) und mit ihrer physischen Situation war zwar immer noch größer als bei der deutschen Allgemeinbevölkerung, allerdings in geringerem Ausmaß als in den zuerst genannten Domänen. Der Unterschied zwischen den Patienten 
unserer Studie und den altersstandardisierten Normwerten zeigte sich dementsprechend in diesen zuletzt genannten Dömanen auch nicht statistisch signifikant.

Der Einfluss der Hauttumorerkrankung auf die Lebensqualität scheint daher geringer zu sein, als dies von uns erwartet worden war. Nennenswert ist, dass auch andere Studien, welche die Lebensqualität von Hauttumorpatienten beurteilten, einen geringen Einfluss der Diagnosestellung bzw. Erkrankung auf die Lebensqualität beobachtet hatten.

So konnten SCHLESINGER-RAAB et al. in ihrer Studie, die deutsche Melanompatienten 2 Jahre nach erfolgter Therapie untersuchten, zeigen, dass sich diese Patienten einer ähnlich guten Lebensqualität erfreuten wie die deutsche Allgemeinbevölkerung. Zwischen 40-50\% der Patienten hatten sogar angegeben, dass sich ihre Lebenseinstellung wie auch ihr Gesundheitsbewusstsein verbessert hatten [65].

Eine aktuellen Studie von JAIN et al., in der Patienten mit nicht-melanozytärem Hautkrebs vor und nach operativer Therapie untersucht wurden, weist ähnliche Ergebnisse auf: die Lebensqualität der Patienten hatte sich nach operativer Therapie sogar verbessert [40].

In unserem Fall erstaunt allerdings, dass signifikant bessere Werte erzielt wurden, als dies bei der deutschen Allgemeinbevölkerung der Fall war. Es liegt also nahe, dass es noch weitere Einflussfaktoren gab, die bei der Interpretation der durch uns erhobenen Ergebnisse berücksichtigt werden sollten.

So scheint eine Verzerrung der Daten durch regionale Effekte möglich. ANGERMEYER et al. erklärte, dass Umweltbedingungen sich signifikant auf die Globallebensqualität auswirken können [2]. Es ist also vorstellbar, dass die saarländische Bevölkerung von Grund auf subjektiv eine höhere Lebensqualität empfindet als die deutsche Allgemeinbevölkerung. Isolierte Vergleichsdaten der gesunden saarländischen Allgemeinbevölkerung, um dies zu prüfen, lagen uns leider nicht vor.

Ein weiterer zu berücksichtigender Faktor ist der von uns verwendete Fragebogen (WHOQOLBREF), der die Effekte, welche Hautkrebs auf die Lebensqualität hat, ggf. nicht spezifisch genug erfasst.

So sahen auch BURDEN-JONES und GIBBONS ein Problem darin, Fragebögen zu verwenden, welche die globale Lebensqualität zwar erfassten, jedoch vielleicht für andere Krankheitsbilder konzipiert worden waren. Im Rahmen einer Studie hatten sie einen Fragebogen („Skin Cancer Quality of Life Impact Tool“) validiert, mit dem sich die Lebensqualität von Patienten mit nicht- 
metastasiertem Hautkrebs untereinander vergleichen ließ [14]. Unseres Wissens existiert solch ein spezifischer Bogen zur Erfassung der Lebensqualität von Hautkrebspatienten allerdings nicht in deutscher Sprache.

Auch WALDMANN et al. hegten Zweifel, ob der in ihrem Fall verwendete Fragebogen zur Erfassung der Lebensqualität bei Krebspatienten (EORTCQLQ-C30) die krankheitsspezifischen Faktoren des Hautkrebses ausreichend erfasst hatte. In ihrer Studie waren 450 deutsche Melanompatienten 15 Monate nach Diagnosestellung, und ein zweites Mal 2 Jahre nach dem ersten Fragebogen, befragt worden. Im Vergleich der Melanompatienten zur deutschen Allgemeinpopulation hatten bezüglich der Lebensqualität keine Unterschiede beobachtet werden können. Ebenso wenig konnte eine Abhängigkeit der Lebensqualität von Tumorgröße oder Tumorlokalisation nachgewiesen werden. WALDMANN et al. vermuteten, dass die Patienten, die erst 15 Monate nach Diagnosestellung zu ihrer Lebensqualität befragt wurden, sich inzwischen an ihre Situation gewöhnt und ihre Antworten ihrem aktuellen Empfinden entsprechend angepasst hatten [81].

Schon HOWARD und DAILEY hatten diese Erscheinung ('response shift') bereits 1979 beschrieben. Hierbei änderte sich nach einem einschneidenden Erlebnis, beispielsweise der Diagnose einer Krebserkrankung, die Einstellung des Patienten zum Positiven [38].

Ein ähnlicher Effekt ist daher auch in unserer Studie vorstellbar. Da unsere Daten retrospektiv erhoben wurden, ist es möglich, dass unsere Patienten - nach erfolgter Therapie - bereits eine Verbesserung ihrer Lebensqualität verzeichnet hatten. Hier fehlen Vergleichswerte des zeitlichen Verlaufs (z.B. bei Diagnosestellung und 6 Monate danach) um zu klären, wie sich die Lebensqualität unserer Patienten entwickelt hatte.

Eine weitere Limitation ergab sich daraus, dass nicht erfasst wurde, welche Nebendiagnosen unsere Patienten hatten. SCHLESINGER-RAAB et al. sahen eine ausgeprägte Abhängigkeit zwischen Komorbiditäten und empfundener Lebensqualität. Da im Mittelpunkt unserer Studie die Verhaltensänderung nach Diagnose stand, und sich im retrospektiven Studiendesign das Erfassen und Kategorisieren der Komorbiditäten nur unzureichend durchführen ließ, wurden Komorbiditäten nicht berücksichtigt.

Weitere Forschung könnte an den oben genannten Punkten ansetzen und mittels einer prospektiven Studie klären, inwieweit sich die Lebensqualität saarländischer Hautkrebspatienten ab 
Diagnosestellung, bzw. im späteren zeitlichen Verlauf, entwickelt, und in welchen Bereichen die Patienten noch Unterstützung benötigen. Gegebenenfalls sollte hier ein geeigneter krankheitsspezifischer Fragebogen zur Erfassung der Lebensqualität verwendet werden. Erst kürzlich veröffentlichten MÜLLER et al. Ergebnisse, die belegten, dass der oben erwähnte EORTCQLQ-C30 Fragebogen, den auch WALDMANN et al. benutzt hatten, ein geeignetes Instrument zur Beurteilung der Lebensqualität bei Patienten mit nicht melanozytärem Tumoren darstellt [54].

Im Kontrast zu unserer Arbeit gab es aber auch Studien, die eine schlechtere Lebensqualität bei den an Hautkrebs erkrankten Patienten beobachteten. So zeigten CORNISH et al. in ihrem systematischen Literaturreview, dass der Einfluss einer Melanomerkrankung auf die Lebensqualität mit dem Einfluss von anderen Krebsarten vergleichbar war, und dass bei vielen Patienten der erlebte emotionale Stress, insbesondere bei Diagnosestellung und auch in Bezug auf die bevorstehende Behandlung, sehr ausgeprägt war. Am stärksten war dieser Einfluss bei systemischer Behandlung des Melanoms. Vor allem die psychische Stabilität hatte entscheidenden Einfluss auf die krankheitsbezogene Lebensqualität [17]. Beim Vergleich mit unserer Studie sollte hier allerdings beachtet werden, dass unsere Patienten sich zur Teilnahme qualifiziert hatten, indem sie an einem Hauttumor operiert worden waren. Wie viele von Ihnen auch eine systemische Chemotherapie bekommen hatten, wurde nicht erfasst.

Ein von uns erhoffter Ansatzpunkt zur Verbesserung der Compliance auf Grund schlechter Lebensqualität nach Hautkrebsdiagnose ergab sich im Gegensatz zu unseren Erwartungen also nicht. Sollte man vielleicht die subjektiv gute Lebensqualität unserer Patienten als Hinweis dafür betrachten, dass sie die Schwere ihrer Erkrankung nicht hinreichend erfassten und somit gesundheitspräventive Verhaltensweisen nicht angemessen umsetzten? In diesem Zusammenhang ist von Bedeutung, dass lt. RHEE et al. Patienten mit nicht melanozytärem Tumor ihr Risiko, in Zukunft an einem Melanom zu erkranken, völlig unterschätzten [62].

\subsubsection{Geschlechtsspezifische Betrachtung}

In der geschlechtsspezifischen Betrachtung der Lebensqualität sticht hervor, dass Frauen in unserer Studie mit ihrer Lebensqualität in allen Domänen zufriedener waren als Männer. In Bezug auf das soziale Umfeld war der Unterschied besonders ausgeprägt und zeigte hier auch eine statistische Signifikanz.

Dessen ungeachtet waren aber auch die männlichen Patienten zufriedener mit ihrer Lebensqualität als die deutsche Allgemeinbevölkerung. Eine Ausnahme stellte der „Globalwert“ dar. Im Vergleich 
mit den geschlechtsspezifischen, altersstandardisierten Normwerten der deutschen Allgemeinbevölkerung zeigten sich unsere männlichen Patienten marginal unzufriedener mit ihrem Gesundheitszustand.

SCHLESINGER-RAAB et al. beobachteten bei der Lebensqualität der von ihnen untersuchten Melanompatienten ebenfalls nur geringe geschlechtsspezifische Unterschiede, die denen der Allgemeinbevölkerung entsprachen [65].

Im Gegensatz zu unserer Studie sahen HOLTERHUES et al. in ihrer Studie dänischer Melanompatienten, dass die Erkrankung einen größeren Einfluss auf die Lebensqualität von Frauen hatte [37]. Ein ähnlicher Effekt wurde von WALDMANN et al. beschrieben. Weibliche Patienten litten mehr unter den Folgen der Tumorerkrankung und gaben insbesondere Probleme mit emotionaler Instabilität, Schlaflosigkeit und Müdigkeit an [81].

Die Ergebnisse unserer Studie bieten Grund zu der Annahme, dass bei den männlichen Patienten noch Bedarf für eine intensivere ärztliche Betreuung besteht. BURDEN-JONES und GIBBONS zeigten, dass Patienten mit anhaltend schlechter Lebensqualität von zusätzlicher Aufmerksamkeit profitierten [14]. Ein engerer Arzt-Patienten-Kontakt könnte sich insbesondere auf das Sozialleben und den emotionalen Zustand des Patienten positiv auswirken [65].

Allerdings sollte bei der Interpretation unserer Ergebnisse nicht aus dem Blick geraten, dass die männlichen Patienten in keiner der Domänen statistisch signifikant unzufriedener, sondern im Gegenteil eher zufriedener waren als die deutsche Allgemeinbevölkerung.

\subsubsection{Familienstand und Qualifikation}

In unserer Auswertung gemäß Familienstand (mit oder ohne Partner lebend) trat hervor, dass Patienten mit Partner in allen 5 Domänen zufriedener waren als Patienten ohne Partner. Insbesondere traf dies auf die Domäne „Umwelt“ zu und zeigte sich hier auch statistisch signifikant. Allerdings waren auch die Patienten, die ohne Partner lebten, in allen Domänen zufriedener als die deutsche Allgemeinbevölkerung.

Einen ähnlichen Effekt beobachteten GIESE-DAVIS et al. Sie hatten im Rahmen ihrer Studie 1196 Patienten mit unterschiedlichen Krebsdiagnosen (darunter 27.1\% gastrointestinale Tumoren, 19.2\% Prostata-, 11.1\% Haut- und 9.2\% gynäkologische Tumoren) untersucht und festgestellt, dass Patienten, die mit Partner lebten, die psychosozialen Effekte der Tumorerkrankung besser abfangen konnten. Ein Nebeneffekt dieses erfreulichen Umstandes war allerdings das Risiko, dass diese 
Patienten auch ihre Krankheit weniger wahrnahmen und häufiger auf unterstützende Maßnahmen verzichteten [30].

Gemäß Qualifikation war bei beiden Gruppen (niedrigeres wie höheres Bildungsniveau) in allen Domänen eine größere Zufriedenheit mit der Lebensqualität zu beobachten, als dies bei der deutschen Allgemeinbevölkerung der Fall war.

Wie von uns erwartet, waren die Patienten mit höherem Bildungsniveau in fast allen Domänen („Physisch“, „Psychisch“, „Umwelt“ und „Globalwert“) zufriedener, als die Patienten mit niedrigerem Bildungsniveau; allerdings zeigten sich die Unterschiede in keiner Domäne signifikant.

Sowohl in der Betrachtung der Unterschiede gemäß Familienstand als auch Qualifikation zeigten sich nur marginale Diskrepanzen, so dass eine Aussage, ob die eine Gruppe mehr Unterstützung nach einer Hautkrebsdiagnose benötigte als die andere, schwer fällt.

Das Zusammenleben mit einem Partner (ob verheiratet oder mit Partner lebend) scheint zu einer insgesamt besseren Lebensqualität zu führen und kann augenscheinlich auch die negativen Effekte einer Krebsdiagnose abmildern. Auch ein höheres Bildungsniveau scheint zu einer besseren Lebensqualität zu führen, allerdings, wie sich bei der Diskussion des Homburger Gesundheitsbogens noch zeigen wird, nicht zwingend, zu einer besseren Compliance der Patienten.

ROSS und VAN WILLIGEN hatten den Einfluss des Bildungsniveaus auf die Lebensqualität untersucht und herausgefunden, dass gebildetere Personen zwar weniger emotionalen Stress, körperliche Schmerzen und Gebrechen, beklagten. Allerdings konnten ROSS und VAN WILLIGEN bezüglich der allgemeinen Zufriedenheit keine Unterschiede gemäß des Bildungsniveaus erkennen [64].

\subsubsection{Tumorentität}

Auch in der differenzierten Betrachtung entsprechend der Tumorentität, unter der unsere Patienten litten, zeigte sich in jeder der Gruppen (Patienten mit Melanom, mit nicht-melanozytärem Tumor oder beiden Tumoren) durchweg eine höhere Zufriedenheit mit der Lebensqualität als bei der deutschen Allgemeinbevölkerung. Im Vergleich der verschiedenen Patientengruppen untereinander ergaben sich nur geringfügige Unterschiede, und daher ergab sich auch keine statistische Signifikanz.

Bislang existieren nur wenige Daten, welche die Lebensqualität von Patienten mit melanozytärem und nicht-melanozytärem Tumor vergleichen. Wie bereits erwähnt, hatten BURDON-JONES und GIBBONS diesbezüglich im Rahmen einer Studie einen Fragebogen mit dem Ziel validiert, 
insgesamt 120 Patienten nach der Behandlung und im weiteren Verlauf der Erkrankung zu befragen. Es zeigte sich, dass insbesondere die Patienten mit melanozytärem Tumor eine schlechtere Lebensqualität angaben, als solche mit nicht-melanozytärem Tumor [14].

Auch wir hatten bei unseren Patienten einen ähnlichen Effekt erwartet. Gleichwohl stellte sich heraus, dass die Patienten mit einem Melanom nur geringfügig zufriedener mit ihrem allgemeinen Gesundheitszustand („Globalwert“) waren als die deutsche Allgemeinbevölkerung, und etwas unzufriedener als die Patienten mit einem nicht-melanozytärem Tumor ( $\mathrm{p}=0,548)$. , Hingegen waren die Patienten mit Melanom in fast allen anderen Domänen („Umwelt“, „Soziale Beziehung“ und „Psychisch“) zufriedener, als Patienten mit nicht melanozytärem Tumor.

In einer früheren Studie hatten BURDON-JONES et al. Patienten mit nicht-metastasiertem Melanom solchen mit nicht-melanozytärem Tumor gegenübergestellt. Sie konnten zeigen, dass Melanompatienten nach erfolgter Therapie sogar eine Erleichterung verspürten und ihr Leben fortan genießen wollten. Allerdings empfanden sie auch signifikant mehr Angst, Schuld und Stress als Patienten mit nicht-melanozytären Tumoren [15].

\subsubsection{Alter}

Wie bereits einleitend beschrieben, wurden die Normwerte für unsere Gruppe altersstandardisiert. Ein Vergleich zwischen den Altersgruppen war somit nicht mehr möglich. Allerdings lagen uns auch Normwerte der deutschen Allgemeinbevölkerung in den verschiedenen Altersgruppen vor.

Diese konnten wir mit den Mittelwerten unserer Patienten vergleichen mit dem Ziel, altersspezifische Unterschiede in Bezug auf die Lebensqualität zu finden [2].

In dieser Auswertung zeigte sich, dass die jüngeren Patienten (bis 55 Jahre) unzufriedener mit ihrer Lebensqualität waren als die älteren. Eine Ausnahme stellten Patienten zwischen 26-35 Jahren dar, die bis auf den „Globalwert“ (Zufriedenheit mit dem allgemeinen Gesundheitszustand) in allen Domänen zufriedener waren, als die deutsche Allgemeinbevölkerung. Allerdings beinhaltete diese Gruppe insgesamt nur 6 Patienten, sodass man die Aussagekraft dieses Vergleiches kritisch sehen sollte. Gleichwohl lässt sich festhalten, dass gegenüber der altersgleichen deutschen Allgemeinbevölkerung vor allem in den Altersgruppen von 36-45 und 46-55 Jahren in den meisten Domänen eine geringere Lebensqualität verzeichnet wurde. Diese Unzufriedenheit war in diesen beiden Altersgruppen, bezüglich der physischen Konstitution und des psychischen Zustandes, besonders ausgeprägt. Hingegen war in den höheren Altersgruppen, also ab 56, eine stetig steigende Lebensqualität in fast allen Domänen evident, die ihre größte Ausprägung bei den über 85-jährigen 
erfuhr.

Es scheint daher, dass die Krankheit einen größeren Einfluss auf die Lebensqualität der jüngeren Patienten hatte, was andererseits (wie sich in der Diskussion des Homburger Gesundheitsbogens noch zeigen wird), zu einer größeren Compliance in einigen Bereichen der Lebensstilveränderung führte.

BIFULCO et al. untersuchten 263 Patientinnen mit einem gynäkologischen Tumor. Hierbei zeigte sich, das jüngere Patientinnen gehäuft unter verschiedenen Symptomen, wie beispielsweise Müdigkeit und gastrointestinalen Beschwerden, litten, und dass die Krebserkrankung einen größeren Einfluss auf das soziale Leben hatte, als dies bei einer Vergleichsgruppe der Fall war. Die jüngeren Patientinnen hatten offensichtlich eine schlechtere Lebensqualität als die Patientinnen mittleren Alters (welche sich nach Krebsdiagnose und Behandlung besser fühlten). Interessant war, wie sich dies auf eine Änderung der bisherigen Verhaltensweisen auswirkte. Die jüngeren Patientinnen hatten eher das Bedürfnis, etwas zu verändern. Somit konnte eine altersabhängige Veränderung des Lebensstils beobachtet werden [8].

Interessant, dass auch bei uns die Zufriedenheit mit der physischen Verfassung bei den älteren Patienten ausgeprägter war als bei den jüngeren.

Im Gegensatz zu uns hatten SCHLESINGER-RAAB et al. eine bedeutende Abhängigkeit zwischen jungem Lebensalter und guter Lebensqualität, insbesondere in Bezug auf die körperliche Verfassung, beobachtet. Hingegen waren in ihrer Studie eher die älteren Patienten emotional stabil [65].

Bezüglich unserer Auswertung nach den verschiedenen Altersgruppen muss noch eine Limitation erläutert werden. Die Mittelwerte der deutschen Normalbevölkerung (WHOQOL-BREF Werte) nehmen in allen Domänen mit steigendem Lebensalter kontinuierlich ab [2]. Auch die Mittelwerte unserer älteren Patienten in den einzelnen Domänen sind häufig schlechter als bei jüngeren Patienten. Allerdings wurde die Mittelwertdifferenz aus dem Normwert der deutschen Allgemeinbevölkerung einer bestimmten Altersgruppe (z.B. >85 Jahre) und dem Mittelwert unserer Patienten derselben Altersgruppe (auch >85 Jahre) berechnet. Somit ist nur die Aussage zulässig, dass die Lebensqualität der jüngeren Patienten (im Vergleich mit der gleichaltrigen deutschen Normalbevölkerung) geringer ist, als die älterer Patienten (also jeweils im Vergleich mit der gleichaltrigen deutschen Allgemeinbevölkerung). 


\subsection{Homburger Gesundheitsbogen}

\subsubsection{Allgemeine Limitationen des Homburger Gesundheitsbogens}

Bei der Auswertung des Homburger Gesundheitsbogens fiel vor allem im ersten Teil (also bei den Fragen, die mittels Likert-Skala zu beantworten waren) auf, dass Patienten, die sich beispielsweise bereits vor Bekanntwerden ihrer Hautkrebserkrankung viel bewegt hatten, die Frage, ob sie das Maß ihrer körperlichen Bewegung nach Bekanntwerden ihrer Diagnose gesteigert hatten, mit „trifft kaum zu“ oder „trifft gar nicht zu“ beantworteten. Bei der Auswertung zählten diese Patienten daher zu jenen, die ihr Verhalten nicht oder kaum verändert hatten. Man hätte also diesen Patienten die Möglichkeit geben sollen, einen entsprechenden Vermerk zu machen, oder erfassen sollen, wie das Bewegungsverhalten vor der Diagnose gewesen war. In einer künftigen prospektiven Studie sollte demzufolge den Patienten die Möglichkeit gegeben werden, ihr Verhalten vor einer Diagnose einzuordnen, um die Dynamik von Veränderungen zuverlässiger nachzuvollziehen.

Eine weitere Limitation ergab sich, weil die Angaben der Patienten zu Lebensstilveränderungen natürlich subjektiv waren. Generell wäre die objektive Erfassung von Veränderungen im Lebensstil von höherer Aussagekraft gewesen, auf Grund des Studiendesigns war solch eine Erhebung der Daten jedoch nicht vorgesehen.

Der Homburger Gesundheitsbogen wurde eigens für unsere Zwecke entwickelt und abgestimmt. Er wurde nicht in Testverfahren validiert.

Ein Vorteil unserer Studie war, dass der Fragebogen erst im Jahr 2014 durch die Patienten ausgefüllt wurde, wohingegen die Diagnose im Jahr 2013 gestellt worden war. Infolgedessen kann man davon ausgehen, dass die Veränderungen im Lebensstil permanent, und nicht nur temporärer Natur waren.

\subsubsection{Körperliche Bewegung}

Bei der Frage nach körperlicher Bewegung fiel auf, dass ein Großteil unserer Patienten sich nach der Diagnose nicht mehr bewegte als zuvor, (74,8\%; $n=195)$, und auch keine neue Sportart begonnen hatte (89,0\%; $n=232)$. Trotzdem erstaunt, dass immerhin 25,2\% $(n=66)$ angaben, sich mehr zu bewegen als vor der Diagnose ihrer Erkrankung.

Die Ergebnisse unserer Studie decken sich mit denen von RHEE et al., die Patienten mit nicht melanozytärem Hautkrebs untersucht hatten. Auch hier hatte sich die Diagnose nicht signifikant auf die physische Aktivität der Patienten ausgewirkt [62].

Verschiedene Studien konnten bereits die präventive Wirkung von körperlicher Bewegung auf 
unterschiedliche Krebserkrankungen feststellen [66], und in der Tat gibt es Hinweise, dass sich körperliche Aktivität präventiv auf die Entstehung von Melanomen auswirkt. In einer Studie, die dänische Briefträger, welche sich im Rahmen ihrer Arbeit verstärkt bewegten, untersuchte, konnte ein signifikant verringertes Risiko für die Entstehung von Melanomen nachgewiesen werden [66]. Zwar muss die präventive Wirkung von körperlicher Bewegung auf Hautkrebs noch genauer untersucht werden [50, 49], allerdings sollten Patienten insbesondere in Hinsicht auf die positiven Auswirkungen körperlicher Bewegung auf die Lebensqualität [18], in der Nachsorge vermehrt auf den hohen Stellenwert körperlicher Aktivität aufmerksam gemacht werden.

\subsubsection{Vergleich der Subgruppen}

Weder das Geschlecht noch der Familienstand unserer Patienten hatten einen signifikanten Einfluss darauf, ob sie sich zu mehr körperlicher Bewegung oder einer neuen Sportart motiviert fühlten. Auch BLANCHARD et al. - siehe Einleitung - kamen zu ähnlichen Ergebnissen [11].

Zwar hatten weder Grunddiagnose noch Bildungsstand einen signifikanten Einfluss, trotzdem ist erwähnenswert, dass sich mit 28,0\% $(n=55)$ vor allem die Patienten mit niedrigem Bildungsniveau mehr bewegten als solche mit hohem $(18,0 \% ; n=11 ; p=0,051)$. Zudem waren es vornehmlich Patienten, die unter beiden Tumorentitäten litten, die sich mehr bewegten $(38,1 \%$; $=16 ; p=0,095)$, jedoch seltener eine neue Sportart begannen $(7,1 \% ; n=10 ; p=0,055)$.

Da insbesondere die nicht melanozytären Tumoren eine Erkrankung des fortgeschrittenen Lebensalters sind [63], und ein Großteil unserer Patienten unter diesem Tumor litt, stellte sich die Frage, ob die Ergebnisse unserer Studie durch den hohen Altersdurchschnitt unserer Patienten (knapp 70 Jahre) beeinflusst wurden.

Unter den jüngeren (bis 65 Jahre) waren es 4\% mehr als bei den älteren Patienten (ab 66 Jahre), die sich mehr bewegten als noch vor der Diagnose $(\mathrm{p}=0,282)$. Die größte Diskrepanz zeigte sich bei der Frage, ob eine neue Sportart begonnen wurde. Hier fanden sich 18,6\% $(n=16)$ unter den jüngeren gegenüber nur 7,4\% $(n=13)$ bei den älteren Patienten $(p=0,001)$. Die jüngeren hatten signifikant häufiger als die älteren Patienten eine neue Sportart begonnen.

Wie auch die o.a. Studien erkennen lassen, benötigten auch unsere Patienten noch Beratung bezüglich vermehrter körperlicher Aktivität und den daraus erwachsenden positiven Auswirkungen. Information über mögliche Sportangebote, und der Stellenwert körperlicher Aktivität sollte in der Nachsorge einen festen Platz einnehmen. Die Ergebnisse unserer Studie sind ein Hinweis, dass vor 
allem ältere Patienten im Fokus einer eingehenderen Beratung stehen sollten.

Wie bereits in der Einleitung erwähnt, steht dem leider entgegen, dass selbst bei Krebsarten, deren Assoziation mit verminderter körperlicher Bewegung deutlich zum Ausdruck kommt, Lebensstilveränderungen von Patienten nur unzureichend verwirklicht wurden. Im Gegenteil, viele Patienten waren sogar weniger körperlich aktiv als noch vor der Krebserkrankung [10, 11]. Eine intensive Beratung von Patienten rückt aus dieser Sicht noch weiter in den Vordergrund.

Offensichtlich ist vielen Patienten die Beziehung zwischen physischer Aktivität und positiver Auswirkung auf ihren Gesundheitszustand nicht bewusst. Da jedoch Patienten mit Hautkrebs ein erhöhtes Risiko dafür haben, erneut von Hautkrebs, aber auch von Krebsformen anderer Organsysteme heimgesucht zu werden [25], tritt ein gesundheitsförderlicher Lebensstil umso dringlicher zu Tage.

\subsubsection{Sonnenexposition, Sonnenschutz und Freizeitverhalten bzw. Urlaubsverhalten}

Unter Beachtung der Genese von Hautkrebs und der immensen Bedeutung, die der Vermeidung von UV-Exposition zukommt [20], fand die Auseinandersetzung mit den Fragen dieses Themenkomplexes unser besonderes Interesse.

Die Arbeitsgemeinschaft Dermatologische Prävention hebt hervor, dass ein textiler Schutz und insbesondere die Abschirmung (Vermeiden von Exposition) vor UV-Strahlung, dem Schutz durch Sonnencremes überlegen ist. Bei starker Sonneneinstrahlung soll der Aufenthalt im Freien, z.B. um die Mittagszeit, auf ein Minimum reduziert werden. Erledigungen (z.B. Gartenarbeit etc.) sollten am frühen Morgen oder späten Abend durchgeführt werden. Sonnenbrände sind auf keinen Fall akzeptabel. Die Exposition der Haut sollte generell, besonders im Frühjahr oder im Urlaub, langsam und graduiert erfolgen [20].

OWEN et al. untersuchten zufällig ausgewählte Einwohner Nordirlands. Sie fanden, dass ein Großteil der Befragten Hautbräunung immer noch als gesund und attraktiv wahrnahmen [57]. 2017 wurde von Kirchberger et al. anhand einer Google-basierten Analyse das Internet-Sucherverhalten bzgl. der Begriffe „Sonnencreme“ und „Solarium“ im deutschsprachigen Raum mit dem weltweit verglichen. Es konnte leider gezeigt werden, dass in den deutschsprachigen Ländern regional unterschiedlich ein ungünstiges Suchverhalten zu Gunsten „Solarium“ zu beobachten ist. Dies steht im Kontrast zur weltweiten Tendenz, der abnehmenden Suchanfragen zu „Solarium“ und zunehmenden Suchanfragen zu „Sonnencreme“ [45]. 
Betrachtet man die Ergebnisse unserer Studie, so sticht hervor, dass 88,5\% ( $n=232)$ der Patienten ihre Sonnenexposition reduzierten und 90,2\% ( $n=207)$ auch darauf achteten, ihr Haus nicht bei voller Sonneneinstrahlung zu verlassen. Insofern schien den Patienten die Bedeutung der Expositionsprophylaxe nach Diagnose ihrer Hauttumorerkrankung bewusst geworden zu sein - und wurde von den meisten Patienten auch umgesetzt.

Auch RHEE et al., die 211 amerikanische Patienten in einer prospektiven Studie mit nichtmelanozytärem Hautkrebs untersuchten, zeigten, dass Patienten nach erfolgter Behandlung die Sonnenexposition signifikant verminderten (weniger Sonnenexposition um die Mittagszeit, sich mehr im Schatten aufhielten) [62].

Allerdings zeigte sich in Bezug auf Freizeitaktivitäten, dass unsere Patienten ihr Urlaubsverhalten (56,5\%; n=144), Radtouren bzw. Freibadbesuche etc. $(54,4 \% ; n=141)$ nur in etwas mehr als der Hälfte der Fälle angepasst hatten. Auch in Bezug auf die Verwendung von topischem und textilem Sonnenschutz zeigten sich nur moderate Verbesserungen der Verhaltensweisen. 66,8\% (n=181) der Patienten trugen nach Bekanntwerden der Erkrankung häufiger Sonnencreme auf. 58,1\% (n=166) trugen Sonnenhüte, 57,2\% ( $n=150)$ häufiger eine Sonnenbrille.

Die von RHEE et al. untersuchten Patienten benutzten nach erfolgter Behandlung signifikant mehr textilen und topischen Sonnenschutz (Sonnencreme, Sonnenhut, schützende Kleidung) [62]. MEYER et al. untersuchten 198 Patienten mit nicht-melanozytärem Hautkrebs, von denen 73\% angaben, textilen Sonnenschutz zu tragen [53]. Auch in der Studie von MASER et al., die 260 Patienten mit nicht melanozytärem Tumor untersucht hatten, zeigte sich, dass mit 70,1\% etwas mehr Patienten Sonnencreme benutzten als dies in unserer Studie der Fall war [52].

Es erstaunt daher, dass in unserer Studie immer noch Patienten auf die Benutzung von topischem und textilem Sonnenschutz verzichteten; insbesondere, da es sich um Patienten handelte, die bereits mit Hautkrebs diagnostiziert wurden und eben daher eine besondere Motivation, ihr Verhalten zu ändern, haben sollten. Hier besteht offensichtlich dringend weiterer Aufklärungsbedarf. Erfreulicherweise wurde die direkte Exposition mit UV-Licht durch die meisten unserer Patienten vermieden. In der Studie von MASER et al. gaben nur 32,2\% der Befragten an, weniger Zeit draußen zu verbringen [52]. In der Studie von MEYER et al. hingegen sagten 59\%, dass sie Außenaktivitäten um die Mittagszeit meiden würden [53].

Die oft ausgeprägten Unterschiede (auch in den Subgruppen, z.B. männlich vs. weiblich) im 
Vergleich zu anderen Studien machen es unbedingt erforderlich, regionale Unterschiede zu berücksichtigen. So verglichen GILLESPIE et al. Australier und Schotten und konnten dokumentieren, dass Australier mehr Wissen über Hautkrebs hatten und sich dementsprechend auch besser gegen die Entstehung von Hautkrebs schützten, als ihre schottischen Zeitgenossen [31].

Bezüglich des Freizeit- und Urlaubsverhaltens kommt man zu dem Schluss, dass möglicherweise vielen Patienten der von uns geschilderte Zusammenhang zwischen Freizeit und UV-Exposition nicht bewusst geworden war.

\subsubsection{Geschlechtsspezifische Betrachtung}

Im geschlechtsspezifischen Vergleich waren es die Patientinnen, die nach der Diagnose signifikant mehr Sonnencremes verwendeten $(\mathrm{p}=0,001)$. NOVAK et al. untersuchten in ihrer Studie 50 Patienten nach Entfernung einer Hautläsion und kamen zu ähnlichen Ergebnissen [55]. Nun ist bekannt, dass Frauen generell häufiger als Männer Cremes verwenden und dass diese oft bereits einen gewissen Sonnenschutzfaktor aufweisen. Trotzdem sehen AL-DUJAILI et al. weiterhin die Notwendigkeit, Frauen darauf aufmerksam zu machen, dass kosmetische Cremes nicht die Wirksamkeit einer Sonnenschutzcreme aufweisen [1]. In unserer Studie benutzten nur knapp zwei Drittel der Männer (61,3\%; n=100) mehr Sonnencremes, als noch vor der Diagnose. Hier besteht also noch weiterer Beratungsbedarf.

Um durch Sonnencremes einen wirksamen Schutz zu erreichen, sollten $2 \mathrm{mg} / \mathrm{cm}^{2}$ aufgetragen werden. Für ausreichenden Schutz kommen demzufolge je Erwachsenem ca. 30-40ml zur Anwendung [20]. Diesbezüglich betrachteten PETERSEN und WULF mehrere Studien und konnten zeigen, dass von den Teilnehmern nur 20-50\% der empfohlenen Menge benutzt wurde [58]. Dass zu wenig Creme verwendet wird, ist jedoch nicht der einzige Missgriff beim Benutzen von Sonnencremes. So untersuchten PRATT et al. bezüglich der Effektivität des Eincremens des Gesichts Patienten mit einer speziellen Kamera. Sie fanden heraus, dass vor allem die Region um die Augen von den meisten Patienten nur unzureichend eingecremt wurde [60]. Im übrigen vermittelt das Auftragen von Cremes ein falsches Schutzgefühl. Wie bereits herausgestellt, sollten daher textiler Schutz und Expositionsprophylaxe im Vordergrund stehen [20]. Gleichwohl kann aber auch öffentliche Aufklärungsarbeit helfen, den Sonnenschutz mit Sonnencremes zu einem effektiveren Instrument der Vorsorge zu machen [60].

Die von uns gesichteten Studien haben ergeben, dass vor allem Frauen ein besseres gesundheitspräventives Verhalten bezüglich Sonnenexposition aufwiesen [42, 9, 37]. 
In unserer Studie trugen Patientinnen häufiger Sonnenbrillen (65,0\%; n=65) als Patienten (52,5\%; n=85; p=0,008). Im Gegensatz dazu schützten sich eher die Männer gegen direkte Sonnenexposition und trugen mit 74,3\% $(n=121)$ signifikant häufiger einen Sonnenhut als Frauen (45\%; n=45\%; $p<0,001)$. Bezüglich des Freizeit- (Radtouren/Freibadbesuche etc. $(p=0,543)$ ), und Urlaubsverhaltens $(\mathrm{p}=0,701)$, sowie der Exposition mit UV-Strahlung in den Mittagsstunden $(p=0,198)$, ergaben sich keine signifikanten Unterschiede. Erfreulicherweise reduzierten sowohl Männer (87\%; $n=141)$ als auch Frauen (91\%; n=91) die Aufenthaltsdauer in der Sonne deutlich $(\mathrm{p}=0,096)$.

\subsubsection{Tumorentität}

Bei der Betrachtung von Patienten nach Tumorentität gab es bezüglich der Vermeidung von direkter Sonnenexposition, wie auch des topischen und textilen Sonnenschutzes, zwar keine signifikanten Unterschiede, allerdings fiel auf, dass vor allem Patienten, welche unter beiden Tumorentitäten litten, ihr Verhalten änderten. Beispielsweise mieden unter den Melanompatienten 80,8\% ( $\mathrm{n}=63$ ) eine direkte Sonnenexposition vis-à-vis 90,8\% ( $\mathrm{n}=128)$ unter Patienten mit nicht melanozytären Tumor. Bei Patienten mit beiden Tumoren waren es 95,3\% ( $n=41)$.

Es scheint, dass eine Diagnose mit beiden Tumoren (ob zum selben Zeitpunkt oder bereits im Vorfeld) gesundheitsförderndes Verhalten begünstigt. Wenngleich RHEE et al. nur Patienten mit nicht-melanozytären Tumoren untersucht hatten, konnten auch sie eine ähnliche Tendenz feststellen. Patienten, die sich wiederholt zur Hautkrebsbehandlung vorstellten (also bereits früher einen Hauttumor hatten) wiesen ein ausgeprägteres gesundheitsförderndes Verhalten auf als Patienten, die sich erstmalig in Behandlung befanden [62]. Man kann daraus schließen, dass vor allem erstmalig mit der Diagnose Hautkrebs in Kontakt kommende Patienten ausführlich über notwendige Präventivmaßnahmen aufgeklärt werden sollten.

Unseres Wissens gab es bis dato nur wenige Daten, die Patienten getrennt nach Tumorentität bezüglich ihres gesundheitspräventiven Verhaltens verglichen haben. NOVAK et al. hatten 50 Patienten vor und nach Entfernung einer tumorverdächtigen Hautläsion nach ihrem präventiven Gesundheitsverhalten gefragt. Es zeigte sich, dass die Patienten mit nicht-melanozytären Tumoren häufiger Hüte trugen, als solche mit Melanom, hingegen waren es die Melanompatienten, die eher ihre tägliche Exposition mit Sonnenlicht verminderten [55]. In unserer Studie hingegen hatten eher die Patienten mit nicht-melanozytärem Tumor, sowie mit beiden Tumorentitäten, ihre Sonnenexposition vermindert. 
In Hinsicht auf die große Rolle, welche die akkumulierte Sonnenexposition bei der Entstehung von nicht-melanozytären Tumoren spielt [80], ist es erfreulich, dass in dieser Gruppe fast alle Patienten eine weitere UV-Exposition vermieden.

\subsubsection{Qualifikation}

Im Vergleich zu Patienten mit höherer Qualifikation schienen Patienten mit niedrigerem Qualifikationsniveau (bis einschließlich mittlerer Reife) ihre Sonnenexposition (Zeit in der Sonne, Haus zur Mittagszeit verlassen, Urlaubs- und Freizeitaktivitäten mit erhöhter Sonnenexposition) nach Diagnosestellung in einem größeren Ausmaß reduziert zu haben. Auch in Bezug auf den Schutz mit Sonnencremes hatten Patienten mit niedrigerem Qualifikationsniveau (71,7\%; $n=142$ ), angegeben, mehr Sonnencreme zu verwenden als Patienten mit höherem Qualifikationsniveau (58,9\%; n=36). Beim Thema Sonnenschutz mit Hut und Sonnenbrille waren es eher die Patienten mit höherem Qualifikationsniveau, die diese Schutzmaßnahmen nach der Diagnose verwendeten. Allerdings zeigte sich bei unseren Patienten keiner der Unterschiede statistisch signifikant.

KASPARIAN et al. sahen in ihrem systematischen Literaturreview eher Hinweise dafür, dass sich ein höherer Bildungsabschluss positiv auf den persönlichen Sonnenschutz auswirkte [42]. Es kann diskutiert werden, ob die Patienten unserer Studie mit niedrigerem Schulabschluss bereits vor der Tumordiagnose weniger protektive Sonnenschutzmaßnahmen durchführten und somit mehr Raum für Veränderung blieb - das wäre eine Erklärung, warum diese Patientengruppe bei der Frage nach Verhaltensänderung häufiger mit „,trifft zu“ antwortete.

\subsubsection{Familienstand}

Bei Patienten mit Partner wurde sowohl der topische, als auch der textile Sonnenschutz etwas öfter beobachtet. Bei dem Tragen eines Sonnenhutes erwies sich dieser Unterschied, im Vergleich zu den Patienten ohne Partner, auch als statistisch signifikant $(p=0,042)$.

Auch waren es eher die Patienten mit Partner, die ihr Urlaubs- und Freizeitverhalten anpassten ( $\mathrm{p}=0,579$ und $\mathrm{p}=0,465$ ). Bezüglich der zeitlichen Beschränkung der Sonnenexposition (Haus zur Mittagszeit nicht verlassen, Zeit in der Sonne reduzieren) zeigten sich nur marginale, nicht signifikante, Unterschiede.

Ob eine der Gruppen spezielle Beratung bezüglich des protektiven Verhaltens gegenüber UVExposition benötigt, ließ sich bei unseren Patienten nicht eindeutig identifizieren. Tendenziell praktizierten eher die Patienten mit Partner eine Verbesserung ihres gesundheitsförderlichen 
Verhaltens. Möglich ist auch, dass der Patient durch seinen Partner zu einer Verbesserung seines Verhaltens motiviert worden war.

GIESE-DAVIS et al. beobachteten, dass ein Partner die psychosozialen Effekte der Erkrankung abfangen kann [30]. Siehe auch unsere Diskussion des WHOQOL-BREF zu diesem Thema.

\subsubsection{Alter}

In unserer Studie hatten sich zwischen den jüngeren (unter 65) und den älteren Patienten (über 66) einige signifikanten Unterschiede gezeigt. Mit 84,3\% $(n=144)$ bei den älteren waren es deutlich mehr als bei den jüngeren $(72,4 \%$; $n=63)$, die darauf verzichteten, das Haus bei voller Sonneneinstrahlung zu verlassen $(\mathrm{p}=0,013)$. Allerdings kann man davon ausgehen, dass Patienten ab einem gewissen Lebensalter ihre Wohnung ohnehin seltener verlassen als jüngere. Die älteren $(68,8 \% ; n=121)$ trugen signifikant häufiger $(p=0,002)$ Sonnenhüte als die jüngeren Patienten $(51,7 \% n=45)$.

Hingegen trugen statistisch signifikant $(\mathrm{p}=0,003)$ mehr jüngere Patienten $(79,3 \%$; $n=69)$ nach Diagnosestellung Sonnencremes auf, bei den Älteren waren es nur 63,6\% (n=112). Zu den Fragen nach Urlaubsverhalten, Freizeitverhalten oder nach einer Reduzierung der Aufenthaltsdauer in der Sonne zeigten sich zwischen beiden Altersgruppen keine signifikanten Unterschiede.

Auch RHEE et al. hatten beobachtet, dass ältere Patienten seltener das Haus zur Mittagszeit verließen [62]. In dem systematischen Literaturreview von KASPARIAN et al. konnte kein eindeutiger Zusammenhang zwischen Lebensalter und dem Verhalten gegenüber Sonnenschutzmaßnahmen nachgewiesen werden. Allerdings wurde anhand der vorliegenden Literatur vermutet, dass vor allem junge ein schlechteres gesundheitspräventives Verhalten aufwiesen als ältere Erwachsene [42].

Ein solcher Trend ist bei uns nicht greifbar. Wie bereits erwähnt, gibt es Verhaltensweisen, die eher von den älteren, und solche, die eher von den jüngeren Patienten verwirklicht wurden.

Die Interpretation unserer Ergebnisse setzt das Wissen voraus, dass bei der Aufteilung unserer Patienten nach Alter, mit 65 Jahren eine relativ hohe Grenze zwischen „jungen“ und „alten“ Patienten gewählt wurde. Dies war allerdings erforderlich, da sonst die Gruppe der jüngeren Patienten zu klein geworden wäre.

\subsubsection{Hautkrebsvorsorgeuntersuchung}

In unserer Studie gingen vor Bekanntwerden der Tumorerkrankung 34,9\% ( $n=89)$ der Patienten 
regelmäßig zur Hautkrebsvorsorgeuntersuchung. Im Anschluss waren es 89,1\% (n=228). Das Ergebnis zeigte eine hohe statistische Signifikanz $(\mathrm{p}<0,001)$. Aber auch bezüglich der Frequenz zeigte sich eine Zunahme. Vor der Diagnose gingen (von den 34,9\%) 28,4\% ( $n=25)$ öfter als einmal jährlich zur Vorsorge, danach gingen (von den 89,1\%) 77,5\% (n=172) öfter als einmal jährlich. Auch dieses Ergebnis zeigte sich statistisch signifikant $(\mathrm{p}<0,001)$.

Die Kosten für die Hautkrebsvorsorge in Deutschland werden ab dem 35. Lebensjahr von den gesetzlichen Krankenkassen übernommen. Die Leitlinien zur Prävention von Hautkrebs schlagen zudem vor, dass Risikopersonen (z.B. genetische Prädisposition etc.) zur Selbstinspektion angeleitet werden und in regelmäßigen Abständen von einem geschulten Arzt am ganzen Körper inspiziert werden. Das Intervall sollte hierbei individuell, je nach Risikoprofil, festgelegt werden [20]. Laut einer Studie von OLIVERIA et al. betrug die Sensitivität bzw. Spezifität einer durch den Patienten selbst durchgeführten Untersuchung, die der Entdeckung bzw. Veränderung suspekter Nävi dienen sollte, 60,2\% bzw. 96,2\% [56].

Vergleicht man nun diese Vorgaben mit den Daten unserer Studie, erstaunt es, dass unter unseren Patienten über ein Drittel mindestens einmal jährlich zur Vorsorgeuntersuchung gegangen war. Dies erklärt sich a.e. dadurch, dass es sich bei unseren Patienten vielleicht um Risikopatienten handelte, die bereits durch den Hausarzt gescreent wurden und ggf. bereits in fachdermatologische Behandlung übergeben worden waren.

Dem Nachsorgeschema der S3-Leitlinien zum Melanom ist zu entnehmen, dass sich Patienten regelmäßig selbst untersuchen sollten und, je nach Tumorstadium, in den ersten 3 Jahren nach einem Tumor 3-6 monatlich, im Anschluss für mindestens 10 Jahre 6 monatlich bis jährlich zur Nachsorge gehen sollten. Nach 10 Jahren sollte unabhängig vom Tumorstadium immer noch eine jährliche Ganzkörperuntersuchung erfolgen [21]. Ähnliches findet sich in den Leitlinien für Plattenepithelkarzinome bzw. Basalzellkarzinome. Diese Patienten sollten für mindestens 5 Jahre bzw. 3 Jahre halbjährlich kontrolliert werden, bei entsprechendem Risikoprofil auch häufiger und länger. Im Anschluss sollten lebenslang jährliche Ganzkörperuntersuchungen stattfinden [7, 6].

Folglich hatten knapp 90\% unserer Patienten die Kontrollintervalle eingehalten; hiervon waren wiederum knapp 80\% öfter als einmal jährlich zur Nachsorge gegangen. Mithin waren die meisten dem Nachsorgeschema nachgekommen.

Interessant wäre gewesen, wie viele unserer Patienten eine Selbstuntersuchung durchführten. Dies 
hätte allerdings einen sehr voluminösen Fragebogen erfordert; möglicherweise hätten die Patienten auch den Unterschied zwischen Nachsorgeuntersuchung durch einen Arzt, und einer Eigeninspektion ihres Körpers nicht ausreichend zu differenzieren gewusst.

GILLESPIE et al. verglichen Patienten aus Schottland und Australien, von denen einige bereits Hautkrebs gehabt hatten, andere lediglich jemanden mit Hautkrebs kannten. Die Ergebnisse deckten sich mit denen unserer Studie. Patienten, die bereits Hautkrebs gehabt hatten, gingen signifikant häufiger zum Arzt, um suspekte Hautveränderungen untersuchen zu lassen, untersuchten sich aber auch selbst häufiger [31].

Auch MASER et al. hatten untersucht, inwiefern Patienten ihr Verhalten nach operativer Therapie (Mohs micrographic surgery) eines Tumors verändert hatten. 53,3\% dieser Patienten gaben an, sich regelmäßig selbst zu untersuchen, indes suchten nur 28\% ihren Arzt häufiger auf, als vor der Operation - deutlich weniger als in unserem Fall.

Trotzdem fiel im Rahmen unserer Studie auf, dass 10,9\% der Patienten im Anschluss an eine OP nicht zur Hautkrebsnachsorgeuntersuchung gegangen waren. Nur 77,5\% derer, die zur Hautkrebsnachsorge gingen, taten dies öfter als einmal jährlich.

\subsubsection{Vergleich der Subgruppen}

Bei der Betrachtung der Unterschiede zwischen unseren einzelnen Gruppen tritt bezüglich des Alters ein signifikanter Unterschied hervor. Waren es in beiden Altersgruppen vor der Diagnose noch ungefähr gleich viele, die zur Vorsorge gingen, waren es im Anschluss vor allem die Patienten unter 65, die regelmäßig den Arzt aufsuchten (97,6\%; $n=83)$. Bei den älteren Patienten waren es nur 84,8\% ( $n=145)$; $(p=0,001)$. Insbesondere bei älteren Patienten sollte daher auf die Wichtigkeit des Wahrnehmens von Nachsorgeterminen hingewiesen werden.

Auch GILLESPIE et al. hatten vor allem bei jüngeren eine bessere Compliance bei den Vorsorgeuntersuchungen beobachtet als bei älteren Patienten. Bezüglich des Geschlechts ergaben sich in ihrer Studie keine signifikanten Unterschiede [31].

In unserer Studie hatten eher Frauen Untersuchungen, sowohl vor als nach der Diagnose, wahrgenommen, allerdings war auch bei uns dieser Unterschied nicht signifikant (davor, $\mathrm{p}=0,345$; danach, $p=0,151)$. Schulausbildung wie auch Familienstand hatten keinen signifikanten Einfluss auf die Compliance bezüglich Vorsorgeuntersuchungen. Allerdings gab es Hinweise dafür, dass Patienten mit Partner nach einer Tumorerkrankung etwas häufiger und zuverlässiger (über 1x/Jahr) 
zur Vorsorgeuntersuchung gingen, als solche ohne Partner.

Betrachtet man die Patienten entsprechend der Grunddiagnose, so fiel auf, dass vor allem Patienten mit beiden Tumorentitäten (47,6\%; $n=20)$ bereits vor der Diagnose zur Vorsorgeuntersuchung gegangen waren. Bei Patienten mit Melanom waren es hingegen nur 31,2\% ( $n=24)$, und bei Patienten mit nicht-melanozytärem Tumor 33,1\% (n=45). Möglicherweise hatten sich die Patienten, die unter beiden Tumorentitäten litten, auf Grund eines zweiten Tumors für diese Studie qualifiziert, waren jedoch wegen eines ersten Hauttumors in ihrer Vorgeschichte bereits engmaschig an einen Arzt gebunden. Dies würde die hohe Zahl an Patienten erklären, die bereits vor der Diagnose zur Vorsorgeuntersuchung gegangen waren. Nach der Diagnose waren es vor allem Patienten mit Melanom (94,8\%; n=73) und Patienten mit beiden Tumorentitäten (92,5\%; $n=37)$ die zur Nachsorge gingen. Bei den Patienten mit nicht melanozytärem Tumor waren es 84,9\% $(n=118) ;(p=0,062)$. Ein signifikanter Unterschied ergab sich in der Frequenz der Wahrnehmung von Vorsorgeuntersuchungen nach Diagnosestellung. Nur 68,2\% (n=72) der Patienten mit nichtmelanozytärem Tumor gingen häufiger als einmal jährlich zur Vorsorgeuntersuchung. Solche mit Melanom sowie mit beiden Tumorentitäten waren in der Mehrheit (95,8\% bzw. 86,1\%; p<0,001).

Zusammenfassend lässt sich festhalten, dass ein Großteil unserer Patienten den Empfehlungen der Nachsorge nach kam, aber auch bereits im Vorfeld auf eine regelmäßige ärztliche Untersuchung der Haut geachtet hatte. Bei älteren, sowie bei Patienten mit nicht-melanozytären Tumoren sollte eine engmaschigere Anbindung erfolgen.

\subsubsection{Ernährung und Alkoholkonsum}

Wie bereits im Rahmen der Einleitung erwähnt, gibt es Hinweise für und wider einen Zusammenhang zwischen ungesunder Ernährung und der Entstehung von Hauttumoren [39, 75, 28]. Gleiches gilt für den Alkoholkonsum (siehe 2.3.4.2). Allerdings wurde bereits erörtert, dass Hautkrebspatienten ein erhöhtes Risiko für die Entstehung von Tumoren anderer Organsysteme haben [25]. Ein gesunder Lebensstil scheint insofern unabdingbar.

Bei unseren Patienten ernährten sich nach eigener Aussage etwas über die Hälfte (59,2\%; n=154) gesünder und bewusster. Etwas mehr Patienten (60,9\%; n=157) gaben an, weniger Alkohol zu trinken als vor ihrer Tumorerkrankung. Aus unserer Sicht ein erstaunliches Ergebnis, insbesondere wenn man sich die Zahlen aus Kapitel 5.5.3 in Erinnerung ruft: Dort hatten 56,5\% (n=144) ihr Freizeitverhalten angepasst, 58,1\% $(n=166)$ trugen regelmäßig Sonnenhüte, 57,2\% $(n=150)$ trugen regelmäßig eine Sonnenbrille und 66,8\% benutzten häufiger Sonnencreme. 
Hier wird deutlich, dass die Ernährungsumstellung und Reduzierung des Alkoholkonsums für unsere Patienten einen ähnlichen Stellenwert hatte wie die Benutzung topischer oder textiler Sonnenschutzmittel. Ein Ergebnis, das wir in dieser Form nicht erwartet hatten - namentlich nicht in Anbetracht anderer von uns berücksichtigter Studien.

MASER et al. hatten bei 214 Patienten nach dermatochirurgischer Intervention (Mohs Micrographic Surgery) keine signifikanten Veränderungen in ihrer Ernährung oder ihrem Alkoholkonsum beobachtet (beide Kategorien <20\%). FALK et al. hatten schwedische 55-69jährige Hautkrebspatienten untersucht und sie mit vergleichbaren Patienten, die allerdings anstelle von Hautkrebs unter seborrhoischer Keratose litten, verglichen. Es stellte sich heraus, dass Hautkrebs in der Vorgeschichte signifikant häufiger mit einem höheren Alkoholkonsum einherging[24].

Bereits wiederholt wurde die Studie von BLANCHARD et al. zum Vergleich herangezogen. Diese Studie hatte in einer Gruppe von Patienten, die unter einem Melanom litten, nur bei 14,8\% eine gesunde Diät (entsprechend den Empfehlungen: „5-A-Day consumed five servings of fruit and vegetables each day“) beobachtet (insgesamt der niedrigste Wert im Vergleich mit Patienten mit anderen Tumorarten (z.B. Brustkrebs, Prostatakrebs etc.)[10].

\subsubsection{Vergleich der Subgruppen}

Die Unterteilung unserer Patienten in verschiedene Subgruppen mit anschließendem Vergleich deutet auf nur einen signifikanten Unterschied hin. Es waren vor allem Patienten mit niedrigerem Bildungsabschluss, die ihren Alkoholkonsum reduzierten (66\%; $\mathrm{n}=128$ ). Dies sind 17,6\% mehr als bei der Gruppe mit höherem Bildungsabschluss (48,4\%; $n=29)$; $(p=0,02)$. Auch bei der Frage nach einer Ernährungsumstellung waren es eher die Patienten mit niedrigem Bildungsniveau, die mit

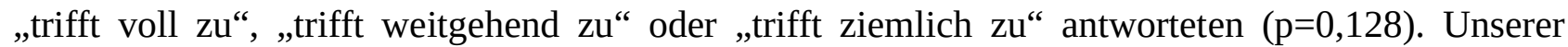
Meinung nach ist dies darin begründet, dass sich die Patienten mit niedrigerem Bildungsstand bereits vor ihrer Erkrankung schlechter ernährt und mehr Alkohol getrunken hatten.

Nun ist weitläufig bekannt, und in diesem Zusammenhang von besonderem Belang, dass Alkoholmissbrauch in Gruppen mit geringerem Bildungsstand höher ist [79, 24]. Diesbezüglich wäre also die Erfragung des Alkoholkonsums unserer Patienten vor der Diagnose angebracht und im nach hinein aufschlussreich gewesen.

Nachfolgend soll noch eine ausgewählte Differenz zwischen unseren Subgruppen erwähnt werden, die wir ungeachtet des Fehlens eines signifikanten Unterschiedes für nennenswert hielten. 
Bereits in Kapitel 5.5.3 waren wir darauf eingegangen, dass Patienten, die unter beiden Tumorentitäten litten, am ehesten bereit waren, ihr Gesundheitsverhalten zu verbessern. Auch bei der Frage nach der Ernährung und dem Alkoholkonsum zeigte sich, dass es wiederum vor allem diese Patienten waren, die ihr Verhalten änderten.

\subsubsection{Nikotinkonsum}

14,8\% ( $n=38$ ) unserer Patienten gaben an, Raucher zu sein bzw. bis zur Diagnosestellung geraucht zu haben. Entsprechend der Zahlen des Statistischen Bundesamtes rauchten beim Mikrozensus 2013 in Deutschland 24,5\% der Befragten. In unserer Studie lag der Altersdurchschnitt bei knapp 70 Jahren. Nach den Daten des Statistischen Bundesamtes für die Altersgruppe der 65-70jährigen rauchen nur noch 15,2\%; denn mit steigendem Lebensalter nimmt der Anteil der Raucher stetig ab. Demzufolge entsprach der Anteil der Raucher unter unseren Patienten in etwa der Anzahl an Rauchern unter der deutschen Allgemeinbevölkerung [70].

Von den 14,8\% ( $n=38)$ der Patienten, die sich als Raucher identifiziert hatten, gaben nur 21,1\% $(n=8)$ an, mit dem Rauchen aufgehört zu haben, 42,1\% ( $n=16)$ hatten das Rauchen nach Diagnosestellung reduziert und 36,8\% $(n=14)$ gaben an, weiterzurauchen.

FALK et al. hatten in ihrer Studie keine Unterschiede bezüglich des Rauchens zwischen den Patienten mit Hautkrebs und der Kontrollgruppe gesehen. In beiden Gruppen rauchten etwas über $10 \%$ [24]. BLANCHARD et al. zeigten, dass insgesamt $11 \%$ der Patienten nach einer Melanomerkrankung rauchten, allerdings wurde nicht erhoben, wie viele der Patienten wegen der Tumorerkrankung mit dem Rauchen aufgehört hatten [10]. Auch MASER et al. beobachteten bei ihren Patienten keine signifikanten Änderungen bezüglich des Rauchverhaltens nach operativem Eingriff [52].

Insbesondere in Hinblick auf die einleitend erläuterte Rolle, die Nikotin bei der Entstehung von Hautkrebs zukommt [67, 35], ist aus unserer Sicht der Anteil an Patienten, die das Rauchen aufgegeben haben, zu gering. Vor allem der Anteil der jungen Raucher ist sehr hoch. So rauchten 36,5\% ( $\mathrm{n}=31$ ) der unter 65jährigen, indes hatten nur 16,1\% nach der Tumorerkrankung aufgehört. Unter den über 66jährigen befanden sich hingegen nur 4,1\% $(n=7)$ Raucher. Von ihnen hatten 42,9\% das Rauchen nach der Diagnose aufgegeben.

\subsubsection{Beruf}

Die Frage, ob ein Beruf mit erhöhter UV-Exposition ausgeübt wird/wurde, bejahten 18,0\% (n=46) 
unserer Patienten. Über die Hälfte (52,2\%; n=24) dieser Patienten gab an, ihren Beruf nach der Diagnose gewechselt zu haben.

Die Bedeutung des Schutzes vor UV-Exposition bei der Arbeit wird auch in den aktuellen Leitlinien zur Prävention von Hautkrebs explizit erwähnt. Es ist Aufgabe des Arbeitgebers, die primären Präventionen umzusetzen [20].

Bei unserer Studie hat uns die hohe Anzahl an Patienten, die angaben, ihren Beruf gewechselt zu haben, erstaunt. Dies sehen wir primär vor dem Hintergrund des Alters der Betroffenen: Zum einen gab es mehr ältere $(n=33)$ als jüngere Patienten $(n=13)$, die einen Beruf mit erhöhter Sonnenexposition angegeben hatten. Zum anderen hatten 60,6\% der über 66jährigen angegeben, ihren Beruf gewechselt zu haben. Bei den jüngeren Patienten (unter 65 Jahren) hatten ihren Beruf indes nur 30,8\% gewechselt. Der Schluss drängt sich auf, dass viele Patienten die Frage nach dem Berufswechsel deshalb bejahen konnten, weil sie sich bereits im Renteneintrittsalter befanden.

\subsection{Fazit}

Soweit bekannt, ist dies die erste Studie, die sich ausführlich mit Verhaltensänderungen saarländischer Patienten nach Hauttumorerkrankungen beschäftigte. Indes spiegelten unsere Ergebnisse weitestgehend auch andere nationale und internationale Studien wider. Sehr erfreulich war die Erkenntnis, dass in Bezug auf Sonnenexposition viele unserer Patienten das empfohlene Verhalten umsetzten.

Auch einer bewussteren Ernährung und Reduzierung des Alkoholkonsums begegnete über die Hälfte unserer der Patienten mit Einsicht und verwirklichte eine Besserung des Lebensstils nach Diagnosestellung. Ein ermutigender Trend, der durch zusätzliche Motivation verstärkt werden sollte.

Die Hautkrebsvorsorge und insbesondere die Nachsorge werden in erfreulich hohem Maße wahrgenommen. Vor allem bei jungen Patienten trifft die Notwendigkeit dieser Schutzmaßnahmen auf gute Resonanz. Gleichzeitig war erkennbar, dass ältere Patientinnen und Patienten mit nichtmelanozytärem Tumor von einer engeren medizinischen Anbindung und größeren Intensität ärztlicher Kontrollen profitieren könnten.

Gleichwohl existieren nach wie vor Bereiche (z.B. textiler Sonnenschutz), sowie andere, von den Patienten nicht direkt in Verbindung mit Hautkrebs gebrachte Risikofaktoren (Rauchen, Bewegung), die eindeutig großen Handlungsbedarf für eine intensivere Aufklärung der Patienten 
erfordern. Auch eine ausführliche Unterweisung erstmals mit Hautkrebs diagnostizierter Patienten in die Tragweite gesundheitsfördernder Verhaltensweisen scheint dringend geboten.

Erstaunlicherweise war die Zufriedenheit mit der Lebensqualität unserer Patienten größer als die der deutschen Allgemeinbevölkerung. Die Auswirkungen der Hautkrebserkrankung auf die Lebensqualität waren geringer als von uns erwartet. In diesem an und für sich erfreulichen Zustand sehen wir einen möglichen Ursprung für den verminderten Antrieb zur Lebensstilveränderung. Die daraus erwachsende Zurückhaltung gegenüber gesundheitsfördernden Veränderungen stellt eine Herausforderung dar.

Weitere Studien wären sinnvoll, um unsere Ergebnisse ggf. zu bestätigen, aber auch um zu klären, in welcher Tiefe Aufklärungsarbeit im Saarland bereits stattfindet. Zudem könnte geklärt werden, welche Maßnahmen sinnvoll wären, um die Compliance der Patienten in den von uns identifizierten Bereichen, zu verbessern. 


\section{Verzeichnisse}

\subsection{Abkürzungsverzeichnis}

Abb.

AWMF

bspw.

bzgl.

bzw.

ca.

etc.

ggf.

i.S.e.

lt.

o.a.

p.a.

PD

Tab.

u.a.

UV

vS.

WHOQOL

z.Zt.
Abbildung

Arbeitsgemeinschaft der Wissenschaftlichen

Medizinischen Fachgesellschaften

beispielsweise

bezüglich

beziehungsweise

circa

et cetera

gegebenenfalls

im Sinne einer

laut

oben aufgeführten

per annum

Privat-Dozent

Tabelle

und andere

ultraviolett

versus

Word Health Organisation Quality of Life

zur Zeit 


\subsection{Abbildungsverzeichnis}

Abbildung 2.1: Todesursachen nach Krankheitsarten 2015 (Statistisches Bundesamt (Destatis), 2017. In www.destatis.de (Thematische Recherche: Zahlen \&

Fakten - Gesellschaft \& Staat - Gesundheit - Todesursachen - Dokumentart:

Abbildung). Abrufdatum: 09.10.2017.................................................................14

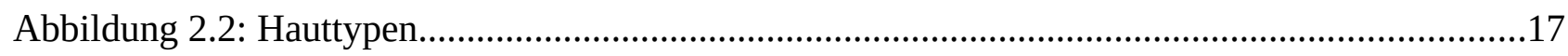

Abbildung 4.1: Patientenübersicht und Rücklaufquote...............................................................30

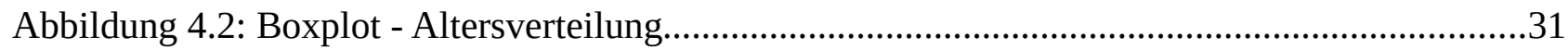

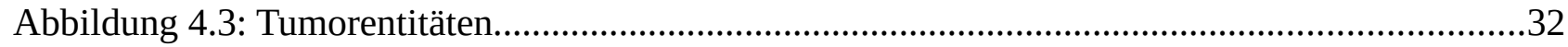

Abbildung 4.4: Tumorentitäten - geschlechtsspezifisch...............................................................32

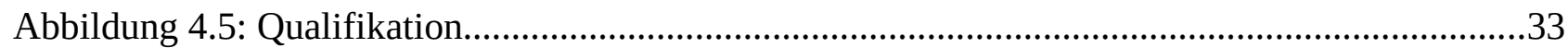

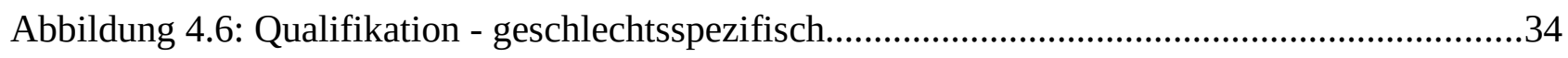

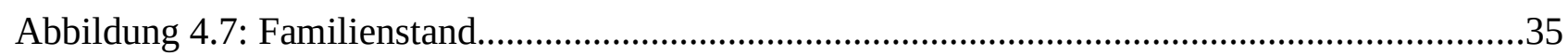

Abbildung 4.8: Familienstand - geschlechtsspezifisch.........................................................35

Abbildung 4.9: Vergleich der Lebensqualität aller Patienten mit den altersstandardisierten

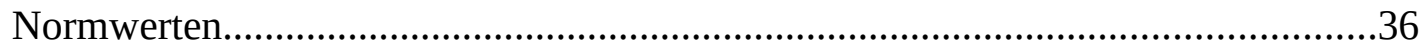

Abbildung 4.10: Vergleich der Lebensqualität von Männern mit den altersstandardisierten Normwerten.

Abbildung 4.11: Vergleich der Lebensqualität von Frauen mit den altersstandardisierten Normwerten. .39

Abbildung 4.12: Vergleich der Lebensqualität gemäß der verschiedenen Altersgruppen..... .43

Abbildung 4.13: Vergleich der Lebensqualität gemäß Bildungsniveau.....

Abbildung 4.14: Vergleich der Lebensqualität von mit Partner lebenden Patienten mit den altersstandardisierten Normwerten.

Abbildung 4.15: Vergleich der Lebensqualität von ohne Partner lebenden Patienten mit den altersstandardisierten Normwerten. .48

Abbildung 4.16: Vergleich der Lebensqualität gemäß Familienstand. .49

Abbildung 4.17: ... bewege ich mich körperlich mehr als vorher...... .52

Abbildung 4.18: ...habe ich eine neue Sportart angefangen, die ich nun regelmäßig verfolge. .52

Abbildung 4.19: ...habe ich eine neue Sportart begonnen, die ich regelmäßig verfolge (Auswertung nach Alter). .57

Abbildung 4.20: ... achte ich mehr darauf, mich nicht lange in der Sonne aufzuhalten. .58 
Abbildung 4.21: ...benutze ich häufiger als früher Sonnenschutzcremes mit Lichtschutzfaktor.......61

Abbildung 4.22: ...trage ich regelmäßig einen Sonnenhut.

Abbildung 4.23: ...benutze ich häufiger als früher Sonnenschutzcremes mit Lichtschutzfaktor (geschlechtsspezifische Auswertung)

Abbildung 4.24: ...trage ich regelmäßig einen Sonnenhut (geschlechtsspezifische Auswertung)....64 Abbildung 4.25: ...trage ich regelmäßig eine Sonnenbrille (geschlechtsspezifische Auswertung)....65 Abbildung 4.26: ...trage ich regelmäßig einen Sonnenhut (Auswertung nach Familienstand)..........69 Abbildung 4.27: ...benutze ich häufiger als früher Sonnenschutzcremes mit Lichtschutzfaktor (Auswertung nach Alter) .70

Abbildung 4.28: ...trage ich regelmäßig einen Sonnenhut (Auswertung nach Alter)........................71

Abbildung 4.29: ...habe ich mein Urlaubsverhalten geändert. .72

Abbildung 4.30: ...vermeide ich es in den Mittagsstunden, bei voller Sonneneinstrahlung, das Haus zu verlassen

Abbildung 4.31: ...habe ich mein Freizeitverhalten (bspw. Freibadbesuche, Radtouren)

verändert.

Abbildung 4.32: ...vermeide ich es in den Mittagsstunden, bei voller Sonneneinstrahlung, das Haus zu verlassen (geschlechtsspezifische Auswertung).

Abbildung 4.33: ...vermeide ich es in den Mittagsstunden, bei voller Sonneneinstrahlung, das Haus zu verlassen (Auswertung nach Alter).

Abbildung 4.34: ...ernähre ich mich gesünder und bewusster......................................................82

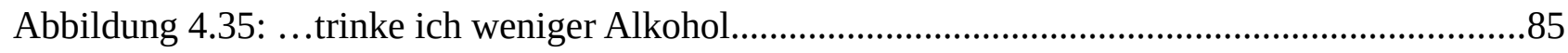

Abbildung 4.36: ...trinke ich weniger Alkohol (Auswertung nach Schulabschluss).........................87

Abbildung 4.37: Sind Sie regelmäßig zur Hautkrebsvorsorgeuntersuchung gegangen?..................91

Abbildung 4.38: Frequenz der Vorsorgeuntersuchung..............................................................91

Abbildung 4.39: Sind Sie regelmäßig zur Hautkrebsvorsorgeuntersuchung gegangen?

(geschlechtsspezifische Auswertung).............................................................92

Abbildung 4.40: Frequenz der Vorsorgeuntersuchung (geschlechtsspezifische Auswertung)...........92

Abbildung 4.41: Sind Sie regelmäßig zur Hautkrebsvorsorgeuntersuchung gegangen?

(Auswertung nach Grunddiagnose)...................................................................93

Abbildung 4.42: Frequenz der Vorsorgeuntersuchung (Auswertung nach der Grunddiagnose)........94

Abbildung 4.43: Sind Sie regelmäßig zur Hautkrebsvorsorgeuntersuchung gegangen? 
Abbildung 4.44: Frequenz der Vorsorgeuntersuchung (Auswertung nach Schulabschluss). .95

Abbildung 4.45: Sind Sie regelmäßig zur Hautkrebsvorsorgeuntersuchung gegangen?

(Auswertung nach Familienstand) ..................................................................96

Abbildung 4.46: Frequenz der Vorsorgeuntersuchung (Auswertung nach Familienstand)...............97

Abbildung 4.47: Sind Sie regelmäßig zur Hautkrebsvorsorgeuntersuchung gegangen?

(Auswertung nach Alter)........................................................................98

Abbildung 4.48: Frequenz der Vorsorgeuntersuchung (Auswertung nach dem Alter)....................98

Abbildung 4.49: Nikotinkonsum nach Diagnose.......................................................................99 


\subsection{Tabellenverzeichnis}

Tabelle 4.1: Vergleich der Lebensqualität aller Patienten mit den altersstandardisierten

Normwerten

Tabelle 4.2: Vergleich der Lebensqualität von Männern mit den altersstandardisierten

Normwerten

Tabelle 4.3: Vergleich der Lebensqualität von Frauen mit den altersstandardisierten

Normwerten .38

Tabelle 4.4: Vergleich der Lebensqualität von Männern und Frauen. .39

Tabelle 4.5: Vergleich der Lebensqualität von 26-35 jährigen mit den Normwerten. .40

Tabelle 4.6: Vergleich der Lebensqualität von 36-45 jährigen mit den Normwerten. .40

Tabelle 4.7: Vergleich der Lebensqualität von 46-55 jährigen mit den Normwerten. .41

Tabelle 4.8: Vergleich der Lebensqualität von 56-65 jährigen mit den Normwerten. .41

Tabelle 4.9: Vergleich der Lebensqualität von 66-75 jährigen mit den Normwerten.

Tabelle 4.10: Vergleich der Lebensqualität von 76-85 jährigen mit den Normwerten.

Tabelle 4.11: Vergleich der Lebensqualität von über 85 jährigen mit den Normwerten.

Tabelle 4.12: Vergleich der Lebensqualität von Patienten mit Melanom mit den altersstandardisierten Normwerten

Tabelle 4.13: Vergleich der Lebensqualität von Patienten mit nicht-melanozytärem Tumor mit den altersstandardisierten Normwerten

Tabelle 4.14: Vergleich Lebensqualität von Patienten mit beiden Tumorentitäten und den altersstandardisierten Normwerten.

Tabelle 4.15: Vergleich der Lebensqualität gemäß verschiedener Tumorentitäten.....

Tabelle 4.16: Vergleich der Lebensqualität von Patienten mit geringerem Bildungsniveau mit den altersstandardisierten Normwerten.....

Tabelle 4.17: Vergleich der Lebensqualität von Patienten mit höherem Bildungsniveau mit den altersstandardisierten Normwerten. .46

Tabelle 4.18: Vergleich der Lebensqualität gemäß Bildungsniveau..... .46

Tabelle 4.19: Vergleich der Lebensqualität von mit Partner lebenden Patienten mit den altersstandardisierten Normwerten

Tabelle 4.20: Vergleich der Lebensqualität von ohne Partner lebenden Patienten mit den altersstandardisierten Normwerten. .49

Tabelle 4.21: Vergleich der Lebensqualität gemäß Familienstand. 
Tabelle 4.22: ...bewege ich mich körperlich mehr als vorher (geschlechtsspezifische

Auswertung).

Tabelle 4.23: ... habe ich eine neue Sportart begonnen, die ich regelmäßig verfolge

(geschlechtsspezifische Auswertung).

Tabelle 4.24: ...bewege ich mich körperlich mehr als vorher (Auswertung nach

Grunddiagnose)

Tabelle 4.25: ...habe ich eine neue Sportart begonnen, die ich regelmäßig verfolge

(Auswertung nach Grunddiagnose).

Tabelle 4.26: ... bewege ich mich körperlich mehr als vorher (Auswertung nach

Schulabschluss)

Tabelle 4.27: ...habe ich eine neue Sportart begonnen, die ich regelmäßig verfolge

(Auswertung nach Schulabschluss).

Tabelle 4.28: ...bewege ich mich körperlich mehr als vorher (Auswertung nach

Familienstand)

Tabelle 4.29: ...habe ich eine neue Sportart begonnen, die ich regelmäßig verfolge

(Auswertung nach Familienstand). .56

Tabelle 4.30: ...bewege ich mich körperlich mehr als vorher (Auswertung nach Alter)

Tabelle 4.31: ...habe ich eine neue Sportart begonnen, die ich regelmäßig verfolge

(Auswertung nach Alter)

Tabelle 4.32: ... achte ich mehr darauf, mich nicht lange in der Sonne aufzuhalten

(geschlechtsspezifische Auswertung)....

Tabelle 4.33: ...achte ich mehr darauf, mich nicht lange in der Sonne aufzuhalten

(Auswertung nach der Grunddiagnose).

Tabelle 4.34: ...achte ich mehr darauf, mich nicht lange in der Sonne aufzuhalten

(Auswertung nach Schulabschluss)....

Tabelle 4.35: ...achte ich mehr darauf, mich nicht lange in der Sonne aufzuhalten

(Auswertung nach Familienstand)

Tabelle 4.36: ...achte ich mehr darauf, mich nicht lange in der Sonne aufzuhalten

(Auswertung nach Alter)

Tabelle 4.37: ...benutze ich häufiger als früher Sonnenschutzcremes mit Lichtschutzfaktor (geschlechtsspezifische Auswertung).

Tabelle 4.38: ...trage ich regelmäßig einen Sonnenhut (geschlechtsspezifische Auswertung). 
Tabelle 4.39: ...trage ich regelmäßig eine Sonnenbrille (geschlechtsspezifische Auswertung).........64

Tabelle 4.40: ...benutze ich häufiger als früher Sonnenschutzcremes mit Lichtschutzfaktor (Auswertung nach der Grunddiagnose)..................................................................65

Tabelle 4.41: ...trage ich regelmäßig einen Sonnenhut (Auswertung nach der Grunddiagnose).......66 Tabelle 4.42: ...trage ich regelmäßig eine Sonnenbrille (Auswertung nach der Grunddiagnose).....66 Tabelle 4.43: ...benutze ich häufiger als früher Sonnenschutzcremes mit Lichtschutzfaktor (Auswertung nach Schulabschluss).

Tabelle 4.44: ...trage ich regelmäßig einen Sonnenhut (Auswertung nach Schulabschluss).....

Tabelle 4.45: ...trage ich regelmäßig eine Sonnenbrille (Auswertung nach dem Schulabschluss)...67

Tabelle 4.46: ...benutze ich häufiger als früher Sonnenschutzcremes mit Lichtschutzfaktor (Auswertung nach Familienstand).

Tabelle 4.47: ...trage ich regelmäßig einen Sonnenhut (Auswertung nach Familienstand)...... .68

Tabelle 4.48: ...trage ich regelmäßig eine Sonnenbrille (Auswertung nach Familienstand).

Tabelle 4.49: ...benutze ich häufiger als früher Sonnenschutzcremes mit Lichtschutzfaktor

(Auswertung nach Alter)

Tabelle 4.50: ...trage ich regelmäßig einen Sonnenhut (Auswertung nach Alter)...... .71

Tabelle 4.51: ...trage ich regelmäßig eine Sonnenbrille (Auswertung nach Alter).....

Tabelle 4.52: ...habe ich mein Urlaubsverhalten geändert (geschlechtsspezifische Auswertung).....73

Tabelle 4.53: ...habe ich mein Urlaubsverhalten geändert (Auswertung nach der Grunddiagnose). .73

Tabelle 4.54: ...habe ich mein Urlaubsverhalten geändert (Auswertung nach Schulabschluss)........74

Tabelle 4.55: ...habe ich mein Urlaubsverhalten geändert (Auswertung nach Familienstand)..........74

Tabelle 4.56: ...habe ich mein Urlaubsverhalten geändert (Auswertung nach Alter).......................75

Tabelle 4.57: ...habe ich mein Freizeitverhalten (bspw. Freibadbesuche, Radtouren) verändert (geschlechtsspezifische Auswertung).....

Tabelle 4.58: ...vermeide ich es in den Mittagsstunden, bei voller Sonneneinstrahlung, das Haus zu verlassen (Auswertung nach der Grunddiagnose).

Tabelle 4.59: ...habe ich mein Freizeitverhalten (bspw. Freibadbesuche, Radtouren) verändert (Auswertung nach der Grunddiagnose).....

Tabelle 4.60: ...vermeide ich es in den Mittagsstunden, bei voller Sonneneinstrahlung, das Haus zu verlassen (Auswertung nach Schulabschluss)... .78

Tabelle 4.61: ...habe ich mein Freizeitverhalten (bspw. Freibadbesuche, Radtouren) verändert (Auswertung nach Schulabschluss). 
Tabelle 4.62: ...vermeide ich es in den Mittagsstunden, bei voller Sonneneinstrahlung,

das Haus zu verlassen (Auswertung nach Familienstand)......

Tabelle 4.63: ...habe ich mein Freizeitverhalten (bspw. Freibadbesuche, Radtouren) verändert

(Auswertung nach Familienstand).

Tabelle 4.64: ...vermeide ich es in den Mittagsstunden, bei voller Sonneneinstrahlung, das Haus zu verlassen (Auswertung nach Alter).

Tabelle 4.65: ...habe ich mein Freizeitverhalten (bspw. Freibadbesuche, Radtouren) verändert

(Auswertung nach Alter)

Tabelle 4.66: ...ernähre ich mich gesünder und bewusster (geschlechtsspezifische Auswertung).....82

Tabelle 4.67: ...ernähre ich mich gesünder und bewusster (Auswertung nach der

Grunddiagnose).

Tabelle 4.68: ...ernähre ich mich gesünder und bewusster (Auswertung nach Schulabschluss)........83

Tabelle 4.69: ...ernähre ich mich gesünder und bewusster (Auswertung nach Familienstand)..........84

Tabelle 4.70: ...ernähre ich mich gesünder und bewusster (Auswertung nach Alter)......................84

Tabelle 4.71: ...trinke ich weniger Alkohol (geschlechtsspezifische Auswertung)..........................85

Tabelle 4.72: ...trinke ich weniger Alkohol (Auswertung nach der Grunddiagnose).......................86

Tabelle 4.73: ...trinke ich weniger Alkohol (Auswertung nach Schulabschluss).............................86

Tabelle 4.74: ...trinke ich weniger Alkohol (Auswertung nach Familienstand)..............................87

Tabelle 4.75: ...trinke ich weniger Alkohol (Auswertung nach Alter)...........................................88

Tabelle 4.76: Berufswechsel (geschlechtsspezifische Auswertung)...............................................88

Tabelle 4.77: Berufswechsel (Auswertung nach der Grunddiagnose).........................................89

Tabelle 4.78: Berufswechsel (Auswertung nach Schulabschluss)................................................89

Tabelle 4.79: Berufswechsel (Auswertung nach Familienstand)................................................89

Tabelle 4.80: Berufswechsel (Auswertung nach Alter)...........................................................90

Tabelle 4.81: Nikotinkonsum (geschlechtsspezifische Auswertung)..........................................99

Tabelle 4.82: Nikotinkonsum (Auswertung nach Grunddiagnose).............................................100

Tabelle 4.83: Nikotinkonsum (Auswertung nach Schulabschluss).............................................100

Tabelle 4.84: Nikotinkonsum (Auswertung nach Familienstand)..............................................100

Tabelle 4.85: Nikotinkonsum (Auswertung nach Alter)............................................................101 


\subsection{Literaturverzeichnis}

1. Al-Dujaili Z, Henry M, Dorizas AS, Sadick NS (2015) Skin cancer concerns particular to women. International journal of women's dermatology 1:123-125.

2. Angermeyer MC, Kilian R, Matschinger H (2000) WHOQOL-100 und WHOQOL-BREF. Handbuch für die deutschsprachige Version der WHO-Instrumente zur Erfasssung von Lebensqualität. Hogrefe, Göttingen Bern Toronto Seattle.

3. Armstrong BK, Kricker A (2001) The epidemiology of UV induced skin cancer. Journal of Photochemistry and Photobiology B: Biology 63:8-18.

4. Augustin M, Amon U, Bullinger M, Gieler U (2000) Empfehlungen zur Erfassung von Lebensqualität in der Dermatologie. Dermatol Psychosom 1:76-82.

5. Autier P, Boniol M, Doré J-F (2007) Sunscreen use and increased duration of intentional sun exposure. Still a burning issue. International journal of cancer. Journal international du cancer 121:1-5.

6. AWMF (2013) Kurzleitlinie - Basalzellkarzinom der Haut (AWMF-Register Nr. 032/02). Online verfügbar unter http://www.awmf.org/uploads/tx_szleitlinien/032-

021l_S2k_Basalzellkarzinom_2013-verlaengert.pdf, zuletzt geprüft am 12.12.2017.

7. AWMF (2013) Kurzleitlinie - Plattenepithelkarzinom der Haut (AWMF-Register Nr. 032/022). Online verfügbar unter http://www.awmf.org/uploads/tx_szleitlinien/032022l_S2k_Plattenepithelkarzinom_2013-verlaengert.pdf, zuletzt geprüft am 12.12.2017.

8. Bifulco G, Rosa N de, Tornesello ML, Piccoli R, Bertrando A, Lavitola G, Morra I, Di Spiezio Sardo A, Buonaguro FM, Nappi C (2012) Quality of life, lifestyle behavior and employment experience. A comparison between young and midlife survivors of gynecology early stage cancers. Gynecologic oncology 124:444-451.

9. Blacam C de, Dermott CM, Sugrue C, Kilmartin D, Kelly J (2017) Patient awareness and sun protection behaviour following excision of basal cell carcinoma. The surgeon : journal of the Royal Colleges of Surgeons of Edinburgh and Ireland 15:12-17.

10. Blanchard CM, Courneya KS, Stein K (2008) Cancer survivors' adherence to lifestyle behavior recommendations and associations with health-related quality of life. Results from the American Cancer Society's SCS-II. Journal of clinical oncology : official journal of the American Society of Clinical Oncology 26:2198-2204.

11. Blanchard CM, Denniston MM, Baker F, Ainsworth SR, Courneya KS, Hann DM, Gesme DH, Reding D, Flynn T, Kennedy JS (2003) Do adults change their lifestyle behaviors after a cancer diagnosis? American journal of health behavior 27:246-256.

12. Bundesamt für Strahlenschutz Wie schütze ich mich vor UV-Strahlung? Online verfügbar unter https://www.bfs.de/DE/themen/opt/uv/sonne/grundlagen/schutz.html, zuletzt geprüft am 10.10.2017.

13. Bundesinstitut für Bevölkerungsforschung Familien und Lebensform. Online verfügbar unter http://www.bibdemografie.de/DE/ZahlenundFakten/12/familien_und_lebensformen_node.html, zuletzt geprüft am 18.01.2018.

14. Burdon-Jones D, Gibbons K (2013) The Skin Cancer Quality of Life Impact Tool 
(SCQOLIT). A validated health-related quality of life questionnaire for non-metastatic skin cancers. Journal of the European Academy of Dermatology and Venereology 27:1109-1113.

15. Burdon-Jones D, Thomas P, Baker R (2010) Quality of life issues in nonmetastatic skin cancer. The British journal of dermatology 162:147-151.

16. Chesnut C, Kim J (2012) Is there truly no benefit with sunscreen use and Basal cell carcinoma? A critical review of the literature and the application of new sunscreen labeling rules to real-world sunscreen practices. Journal of skin cancer 2012:480985.

17. Cornish D, Holterhues C, van de Poll-Franse LV, Coebergh JW, Nijsten T (2009) A systematic review of health-related quality of life in cutaneous melanoma. Annals of oncology : official journal of the European Society for Medical Oncology 20 Suppl 6:51-8.

18. Demark-Wahnefried W, Jones LW (2008) Promoting a healthy lifestyle among cancer survivors. Hematology/oncology clinics of North America 22:319-42.

19. Demark-Wahnefried W, Peterson B, McBride C, Lipkus I, Clipp E (2000) Current health behaviors and readiness to pursue life-style changes among men and women diagnosed with early stage prostate and breast carcinomas. Cancer 88:674-684.

20. Deutsche Krebsgesellschaft, Deutsche Krebshilfe, AWMF (Leitlinienprogramm Onkologie) (2014) S3-Leitlinie Prävention von Hautkrebs (AWMF-Registrierungsnummer: 032/052OL). Online verfügbar unter http://leitlinienprogramm-onkologie.de/Leitlinien.7.0.html, zuletzt geprüft am 28.01.2016.

21. Deutsche Krebsgesellschaft, Deutsche Krebshilfe, AWMF (Leitlinienprogramm Onkologie) (2016) Diagnostik, Therapie und Nachsorge des Melanoms (AWMF Registriernummer: 032/024OL). Online verfügbar unter http://leitlinienprogrammonkologie.de/Melanom.65.0.html, zuletzt geprüft am 09.01.2018.

22. Eisemann N, Waldmann A, Geller AC, Weinstock MA, Volkmer B, Greinert R, Breitbart EW, Katalinic A (2014) Non-melanoma skin cancer incidence and impact of skin cancer screening on incidence. The Journal of investigative dermatology 134:43-50.

23. Eysenbach G, Wyatt J (2002) Using the Internet for surveys and health research. Journal of medical Internet research 4:E13.

24. Falk M, Faresjö A, Faresjö T (2013) Sun exposure habits and health risk-related behaviours among individuals with previous history of skin cancer. Anticancer research 33:631-638.

25. Frisch M, Hjalgrim H, Olsen JH, Melbye M (1996) Risk for subsequent cancer after diagnosis of basal-cell carcinoma. A population-based, epidemiologic study. Annals of internal medicine 125:815-821.

26. Gallagher RP, Hill GB, Bajdik CD, Coldman AJ, Fincham S, McLean DI, Threlfall WJ (1995) Sunlight exposure, pigmentation factors, and risk of nonmelanocytic skin cancer. II. Squamous cell carcinoma. Archives of dermatology 131:164-169.

27. Gallagher RP, Hill GB, Bajdik CD, Fincham S, Coldman AJ, McLean DI, Threlfall WJ (1995) Sunlight exposure, pigmentary factors, and risk of nonmelanocytic skin cancer. I. Basal cell carcinoma. Archives of dermatology 131:157-163.

28. Gamba CS, Stefanick ML, Shikany JM, Larson J, Linos E, Sims ST, Marshall J, van Horn L, Zeitouni N, Tang JY (2013) Low-fat diet and skin cancer risk. The women's health initiative randomized controlled dietary modification trial. Cancer epidemiology, biomarkers \& 
prevention : a publication of the American Association for Cancer Research, cosponsored by the American Society of Preventive Oncology 22:1509-1519.

29. Gesellschaft der epidemiologischen Krebsregister in Deutschland e.V. (Hrsg.) (2012) Atlas der Krebsinzidenz und - mortalität in Deutschland (GEKID-Atlas). Online verfügbar unter www.gekid.de, zuletzt geprüft am 28.01.2016.

30. Giese-Davis J, Waller A, Carlson LE, Groff S, Zhong L, Neri E, Bachor SM, AdamykSimpson J, Rancourt KMS, Dunlop B, Bultz BD (2012) Screening for distress, the 6th vital sign. Common problems in cancer outpatients over one year in usual care: associations with marital status, sex, and age. BMC cancer 12:441.

31. Gillespie HS, Watson T, Emery JD, Lee AJ, Murchie P (2011) A questionnaire to measure melanoma risk, knowledge and protective behaviour. Assessing content validity in a convenience sample of Scots and Australians. BMC medical research methodology 11:123.

32. Green A, Williams G, Neale R, Hart V, Leslie D, Parsons P, Marks GC, Gaffney P, Battistutta D, Frost C, Lang C, Russell A (1999) Daily sunscreen application and betacarotene supplementation in prevention of basal-cell and squamous-cell carcinomas of the skin: a randomised controlled trial. Lancet (London, England) 354:723-729.

33. Green AC, Williams GM, Logan V, Strutton GM (2011) Reduced melanoma after regular sunscreen use: randomized trial follow-up. Journal of clinical oncology : official journal of the American Society of Clinical Oncology 29:257-263.

34. Han J, Colditz GA, Hunter DJ (2006) Risk factors for skin cancers: a nested case-control study within the Nurses' Health Study. International journal of epidemiology 35:1514-1521.

35. Hertog SA de, Wensveen CA, Bastiaens MT, Kielich CJ, Berkhout MJ, Westendorp RG, Vermeer BJ, Bouwes Bavinck JN (2001) Relation between smoking and skin cancer. Journal of clinical oncology : official journal of the American Society of Clinical Oncology 19:231238.

36. Hess DR (2004) Retrospective studies and chart reviews. Respiratory care 49:1171-1174.

37. Holterhues C, Cornish D, van de Poll-Franse, Lonneke, Veronique, Krekels G, Koedijk F, Kuijpers D, Coebergh JW, Nijsten T (2011) Impact of melanoma on patients' lives among 562 survivors. A Dutch population-based study. Archives of dermatology 147:177-185.

38. Howard GS, Dailey PR (1979) Response-shift bias. A source of contamination of self-report measures. Journal of Applied Psychology 64:144-150.

39. Ibiebele TI, van der Pols, Jolieke C, Hughes MC, Marks GC, Williams GM, Green AC (2007) Dietary pattern in association with squamous cell carcinoma of the skin: a prospective study. The American journal of clinical nutrition 85:1401-1408.

40. Jain S, Jain P, Singh A, Goel S, Goyal P, Tank R (2016) An evaluation of quality of life of skin cancer patients after surgery using dermatology life quality index tool. Int J Res Med Sci:3235-3239.

41. Jensen A, Birch-Johansen F, Olesen AB, Christensen J, Tjønneland A, Kjær SK (2012) Intake of alcohol may modify the risk for non-melanoma skin cancer: results of a large Danish prospective cohort study. The Journal of investigative dermatology 132:2718-2726.

42. Kasparian NA, McLoone JK, Meiser B (2009) Skin cancer-related prevention and screening behaviors. A review of the literature. Journal of behavioral medicine 32:406-428. 
43. Katalinic A, Kunze U, Schäfer T (2003) Epidemiology of cutaneous melanoma and nonmelanoma skin cancer in Schleswig-Holstein, Germany: incidence, clinical subtypes, tumour stages and localization (epidemiology of skin cancer). The British journal of dermatology 149:1200-1206.

44. King MF, Bruner GC (2000) Social desirability bias. A neglected aspect of validity testing. Psychol. Mark. 17:79-103.

45. Kirchberger MC, Kirchberger LF, Eigentler TK, Reinhard R, Berking C, Schuler G, Heinzerling L, Heppt MV (2017) Interest in tanning beds and sunscreen in German-speaking countries. Journal der Deutschen Dermatologischen Gesellschaft 15:1192-1198.

46. Kraywinkel K, Bertz J, Laudi A, Wolf U (2012) Epidemiologie und Früherkennung häufiger Krebserkrankungen in Deutschland. Robert-Koch-Institut (GBE kompakt). Online verfügbar unter

http://www.rki.de/DE/Content/Gesundheitsmonitoring/Gesundheitsberichterstattung/GBEDo wnloadsK/2012_4_Krebserkrankungen.pdf?_blob=publicationFile, zuletzt geprüft am 09.10.2017.

47. Kraywinkel K, Wolf U, Katalinic A (2012) Hautkrebs - Epidemiologie und Früherkennung. UMID - Umwelt und Mensch:30-34.

48. Krengel S, Hauschild A, Schäfer T (2006) Melanoma risk in congenital melanocytic naevi. A systematic review. The British journal of dermatology 155:1-8.

49. Lahmann PH, Russell A, Green AC (2011) Prospective study of physical activity and risk of squamous cell carcinoma of the skin. BMC cancer 11:516.

50. Lee TK, MacArthur AC, Gallagher RP, Elwood MJ (2009) Occupational physical activity and risk of malignant melanoma. The Western Canada Melanoma Study. Melanoma research 19:260-266.

51. Marehbian J, Colt JS, Baris D, Stewart P, Stukel TA, Spencer SK, Karagas MR (2007) Occupation and keratinocyte cancer risk. A population-based case-control study. Cancer causes \& control : CCC 18:895-908.

52. Maser E, Berg D, Solish N (2001) Changes in patient perception and behavior following Mohs micrographic surgery. Journal of cutaneous medicine and surgery 5:14-17.

53. Meyer N, Pruvost-Balland C, Bourdon-Lanoy E, Maubec E, Avri M-F (2007) Awareness, knowledge and attitudes towards sun protection among skin cancer-treated patients in France. Journal of the European Academy of Dermatology and Venereology : JEADV 21:520-525.

54. Müller K, Karrer S, Szeimies R-M, Steinbauer J, Kohl E, Steinbauer D, Zeman F, Berneburg M, Koller M (2017) Beurteilung der Lebensqualität bei Patienten mit nicht-melanozytärem Hautkrebs - psychometrische Validierung des EORTC QLQ-C30-Fragebogens. Journal der Deutschen Dermatologischen Gesellschaft 15:1090-1102.

55. Novak CB, Young DS, Lipa JE, Neligan PC (2007) Evaluation of sun protection behaviour in patients following excision of a skin lesion. The Canadian journal of plastic surgery $=$ Journal canadien de chirurgie plastique 15:38-40.

56. Oliveria SA, Chau D, Christos PJ, Charles CA, Mushlin AI, Halpern AC (2004) Diagnostic accuracy of patients in performing skin self-examination and the impact of photography. Archives of dermatology 140:57-62. 
57. Owen T, Fitzpatrick D, Dolan O, Gavin A (2004) Knowledge, attitudes and behaviour in the sun. The barriers to behavioural change in Northern Ireland. The Ulster medical journal 73:96-104.

58. Petersen B, Wulf HC (2014) Application of sunscreen--theory and reality. Photodermatology, photoimmunology \& photomedicine 30:96-101.

59. Petres J, Rompel R (2007) Operative Dermatologie. Lehrbuch und Atlas. 2., erweiterte und vollständig überarbeitete Auflage. Springer, Berlin Heidelberg.

60. Pratt H, Hassanin K, Troughton LD, Czanner G, Zheng Y, McCormick AG, Hamill KJ (2017) UV imaging reveals facial areas that are prone to skin cancer are disproportionately missed during sunscreen application. PloS one 12:e0185297.

61. Rausch SM, Millay S, Scott C, Pruthi S, Clark MM, Patten C, Stan D, Sellers T, Vachon C (2012) Health behaviors among cancer survivors receiving screening mammography. American journal of clinical oncology 35:22-31.

62. Rhee JS, Davis-Malesevich M, Logan BR, Neuburg M, Burzynski M, Nattinger AB (2008) Behavior modification and risk perception in patients with nonmelanoma skin cancer. WMJ : official publication of the State Medical Society of Wisconsin 107:62-68.

63. Robert Koch-Institut (Hrsg.) und die Gesellschaft der epidemiologischen Krebsregister in Deutschland e.V. (Hrsg.) (2017) Krebs in Deutschland für 2013/2014. 11. Ausgabe, Berlin.

64. Ross CE, van Willigen M (1997) Education and the subjective quality of life. Journal of health and social behavior 38:275-297.

65. Schlesinger-Raab A, Schubert-Fritschle G, Hein R, Stolz W, Volkenandt M, Hölzel D, Engel J (2010) Quality of life in localised malignant melanoma. Annals of oncology : official journal of the European Society for Medical Oncology 21:2428-2435.

66. Soll-Johanning H, Bach E (2004) Occupational exposure to air pollution and cancer risk among Danish urban mail carriers. Etiologic evidence and biological mechanisms. International archives of occupational and environmental health 77:351-356.

67. Song F, Qureshi AA, Gao X, Li T, Han J (2012) Smoking and risk of skin cancer: a prospective analysis and a meta-analysis. International journal of epidemiology 41:16941705.

68. Stang A, Stausberg J, Boedeker W, Kerek-Bodde H, Jöckel K-H (2008) Nationwide hospitalization costs of skin melanoma and non-melanoma skin cancer in Germany. Journal of the European Academy of Dermatology and Venereology 22:65-72.

69. Statistisches Bundesamt (destatis.de) Bildungsstand. Bevölkerung nach Bildungsabschluss in Deutschland. Online verfügbar unter www.destatis.de/DE/ZahlenFakten/GesellschaftStaat/BildungForschungKultur/Bildungsstand/ Tabellen/Bildungsabschluss.html, zuletzt geprüft am 18.01.2018.

70. Statistisches Bundesamt (destatis.de) Rauchgewohnheiten nach Altersgruppen und Geschlecht. Ergebnisse des Mikrozensus 2013. Online verfügbar unter https://www.destatis.de/DE/ZahlenFakten/GesellschaftStaat/Gesundheit/GesundheitszustandR elevantesVerhalten/Tabellen/Rauchverhalten.html, zuletzt geprüft am 19.01.2018.

71. Statistisches Bundesamt (destatis.de) (2013) Krebs immer häufiger Todesursache. Online verfügbar unter 
https://www.destatis.de/DE/PresseService/Presse/Pressemitteilungen/2013/02/PD13_042_232 pdf.pdf?_blob=publicationFile, zuletzt geprüft am 09.10.2017.

72. Statistisches Bundesamt (destatis.de) (2013) Todesursachen in Deutschland 2013. Online verfügbar unter https://www.destatis.de/DE/Publikationen/Thematisch/Gesundheit/Todesursachen/Todesursac hen2120400137004.pdf?__blob=publicationFile, zuletzt geprüft am 09.10.2017.

73. Statistisches Bundesamt (destatis.de) (2015) Bildungsstand der Bevölkerung. Online verfügbar unter www.destatis.de/DE/Publikationen/Thematisch/BildungForschungKultur/Bildungsstand/Bildu ngsstandBevoelkerung5210002157004.pdf?_blob=publicationFile, zuletzt geprüft am 19.01.2018.

74. Statistisches Bundesamt (destatis.de) (2015) Todesursachen in Deutschland 2015. Online verfügbar unter https://www.destatis.de/DE/Publikationen/Thematisch/Gesundheit/Todesursachen/Todesursac hen2120400157004.pdf?_blob=publicationFile, zuletzt geprüft am 09.10.2017.

75. Tang JY, Henderson MT, Hernandez-Boussard T, Kubo J, Desai M, Sims ST, Aroda V, Thomas F, McTiernan A, Stefanick ML (2013) Lower skin cancer risk in women with higher body mass index: the women's health initiative observational study. Cancer epidemiology, biomarkers \& prevention : a publication of the American Association for Cancer Research, cosponsored by the American Society of Preventive Oncology 22:2412-2415.

76. Tofthagen C (2012) Threats to validity in retrospective studies. Journal of the advanced practitioner in oncology 3:181-183.

77. Tüchler H, Lutz D (1991) Lebensqualität und Krankheit. Auf dem Weg zu einem medizinischen Kriterium Lebensqualität. Deutscher Ärzte-Verlag, Köln.

78. Valenti M, Porzio G, Aielli F, Verna L, Cannita K, Manno R, Masedu F, Marchetti P, Ficorella C (2008) Physical exercise and quality of life in breast cancer survivors. International journal of medical sciences 5:24-28.

79. van Oers JA, Bongers IM, van de Goor LA, Garretsen HF (1999) Alcohol consumption, alcohol-related problems, problem drinking, and socioeconomic status. Alcohol and alcoholism (Oxford, Oxfordshire) 34:78-88.

80. Vries E de, Trakatelli M, Kalabalikis D, Ferrandiz L, Ruiz-de-Casas A, Moreno-Ramirez D, Sotiriadis D, Ioannides D, Aquilina S, Apap C, Micallef R, Scerri L, Ulrich M, Pitkänen S, Saksela O, Altsitsiadis E, Hinrichs B, Magnoni C, Fiorentini C, Majewski S, Ranki A, Stockfleth E, Proby C (2012) Known and potential new risk factors for skin cancer in European populations: a multicentre case-control study. The British journal of dermatology 167 Suppl 2:1-13.

81. Waldmann A, Nolte S, Pritzkuleit R, Breitbart EW, Katalinic A (2011) Different aspects of self-reported quality of life in 450 german melanoma survivors. Cancers 3:2316-2332.

82. Warthan MM, Sewell DS, Marlow RA, Warthan ML, Wagner RF (2003) The economic impact of acute sunburn. Archives of dermatology 139:1003-1006.

83. World Health Organisation (2017) Cancer. Fact Sheet. Online verfügbar unter www.who.int/mediacentre/factsheets/fs297/en, zuletzt geprüft am 10.10.2017. 
84. Wu S, Han J, Laden F, Qureshi AA (2014) Long-term ultraviolet flux, other potential risk factors, and skin cancer risk: a cohort study. Cancer epidemiology, biomarkers \& prevention : a publication of the American Association for Cancer Research, cosponsored by the American Society of Preventive Oncology 23:1080-1089.

85. Wu S, Li W-Q, Qureshi AA, Cho E (2015) Alcohol consumption and risk of cutaneous basal cell carcinoma in women and men. 3 prospective cohort studies. The American journal of clinical nutrition 102:1158-1166.

\subsection{Eigene und geplante Publikationen}

- Hoernecke DPW, Wagenpfeil S, Vogt T, Müller CSL. Evaluation of Health Behavior and Quality of Life dermato oncology Patients after dermatosurgical Interventions. Journal der deutschen dermatologischen Gesellschaft. Meeting Abstract: FV11/10 Volume: 13 Pages: 107-108 Supplement: 1 Published: APR 2015

- Hoernecke, DPW; Wagenpfeil, S; Pföhler C, Vogt, T; Müller, CSL. Evaluation des Gesundheitsverhaltens und der Lebensqualität dermatoonkologischer Patienten nach dermatochirurgischen Interventionen. Manuscript in preparation. 


\section{Danksagung}

Meine besondere Dankbarkeit gilt meiner Doktormutter Fr. PD Dr. Cornelia Müller für die Überlassung des Themas und die kompetente, zuverlässige und äußerst freundschaftliche Unterstützung während der gesamten Promotion.

Ich danke dem gesamten dermatologischen Institut der Universitätsklinik des Saarlandes um Prof. Dr. med. Vogt für ihre Hilfe.

Zudem danke ich dem Biometrischen Institut der Universität des Saarlandes um Prof. Dr. Wagenpfeil für die Unterstützung bei der statistischen Auswertung. Insbesondere möchte ich hier Herrn Daniel Rapp erwähnen.

Ich danke allen Patienten, die sich die Zeit genommen haben, die Fragebögen auszufüllen.

Meinen Eltern, Detlef und Christa, danke ich von ganzem Herzen für die unermessliche Unterstützung während des gesamten Studiums sowie für die interessanten Anregungen und Ideen beim Verfassen dieser Arbeit.

Meiner Ehefrau, Sabine, möchte ich für die liebevolle Unterstützung, unermüdliche Motivation und die unerschöpfliche Hilfe danken.

Zudem möchte ich mich bei meinem Bruder Carl-Christian mit Ehefrau Sarah bedanken, mit denen ich das Glück hatte, in Homburg/Saar gemeinsam studieren zu dürfen und die mir stets zur Seite standen. 


\section{Lebenslauf}

Aus datenschutzrechtlichen Gründen findet sich der Lebenslauf nur in der gedruckten Version. 


\title{
9 Anhang
}

\subsection{Anschreiben an Patienten}

\author{
Sehr geehrter Herr / Frau XXX
}

In Zusammenarbeit mit der Dermatologischen Klinik der Universität des Saarlandes führe ich zur Zeit eine Untersuchung zum Therapieerfolg bei Hautkrebserkrankungen (z.B. Melanome, etc.) durch. Mit ihrer Teilnahme können sie einen massgeblichen Beitrag zur Verbesserung bestehender Therapien leisten.

Nach Aufzeichnungen der Deutschen Krebshilfe hat sich die Zahl der Hautkrebsfälle in den letzten 10 Jahren verdoppelt. Jährlich erkranken etwa 234.000 Menschen in Deutschland neu an einem Tumor der Haut. Eine wesentliche Ursache ist die vermehrte Sonnenexposition unserer Gesellschaft.

Mit beiliegender Erhebung befragen wir alle bei uns im Jahr 2013 stationär behandelten Patienten. Wir möchten ermitteln, ob und wie sich unsere Empfehlungen bezüglich Verhaltensänderungen in der Praxis umsetzen ließen. Ziel ist, festzustellen, ob eine Veränderung bezüglich der Behandlung (und vor allem der Aufklärung nach erfolgter Behandlung) unsere Therapie weiter verbessern können. Von den Ergebnissen dieser Arbeit, die anonymisiert in meine Promotionsarbeit (Doktorarbeit) an der Dermatologischen Klinik der Universität des Saarlandes einfliessen, erhoffen wir uns Rückschlüsse auf mögliche Therapieverbesserungen.

Ihre Antworten werden verschlüsselt (pseudonymisiert), sodass sie für Dritte nicht nachzuvollziehen sind und die Ergebnisse der Auswertung werden selbstverständlich vollständig anonymisiert ausgewertet. Ihre künftige Therapie, falls erforderlich, wird davon in keiner Weise beeinflusst.

Ihre Gesundheit liegt uns am Herzen. Sollten Sie Fragen zu dieser Untersuchung haben oder an den Ergebnissen dieser Arbeit interessiert sein, können Sie uns unter der unten angegebenen Adresse erreichen.

Bitte senden Sie uns die ausgefüllten Fragebögen möglichst bis zum 31.08.2014 auf postalischem Wege an:

PD Dr. Cornelia Müller

Klinik für Dermatologie, Venerologie und Allergologie, Geb. 18

Universitätsklinikum

66424 Homburg Saar

oder per Fax an PD Dr. Müller 06841/1613816 zurück.

Mit freundlichen Grüßen

Detlef Hoernecke 


\subsection{Homburger Gesundheitsbogen}

Es handelt sich um zwei Fragebögen, die zum einen Ihre Lebensqualität (WHOQOL-BREF Fragebogen) erfassen und zum anderen feststellen sollen wie Sie Ihren Lebensstil seit Bekanntwerden Ihrer Erkrankung verändert haben.

\section{Fragebogen 1 (Homburger Gesundheitsbogen)}

- Dieser Test dauert ca. 5-8min

- Die Fragen des Fragebogens lassen sich beantworten, indem Sie die vorgegebenen Kästchen ankreuzen.

- Bitte machen Sie pro Zeile nur ein Kreuz in die vorgesehenen Kästchen! Entscheiden Sie sich bitte für eine der Antwortmöglichkeiten.

- Wenn Sie Anmerkungen zu den Fragen haben, können Sie diese auf der letzten Seite des Fragebogens notieren.

- Bitte beantworten Sie jede Frage.

- Kreuzen Sie bitte die Antwort an, die am ehesten auf Sie zutrifft.

Bitte machen Sie im folgenden in jeder Zeile jeweils nur ein Kreuz. 
Welche Aussagen trifft am ehesten auf sie zu...

1. Seit Bekanntwerden meiner Hautkrebserkrankung....

\begin{tabular}{|c|c|c|c|c|c|}
\hline $\begin{array}{l}\text {...bewege ich mich } \\
\text { körperlich mehr als vorher }\end{array}$ & $\begin{array}{c}\text { Trifft } \\
\text { voll zu } \\
\square\end{array}$ & $\begin{array}{c}\text { Trifft weitgehend } \\
\text { zu } \\
\square\end{array}$ & $\begin{array}{c}\text { Trifft } \\
\text { ziemlich zu } \\
\square\end{array}$ & $\begin{array}{c}\text { Trifft kaum } \\
\text { zu } \\
\square\end{array}$ & $\begin{array}{c}\text { Trifft gar } \\
\text { nicht zu } \\
\square\end{array}$ \\
\hline $\begin{array}{l}\text {...habe ich eine neue } \\
\text { Sportart angefangen, die ich } \\
\text { nun regelmäßig verfolge }\end{array}$ & $\begin{array}{c}\text { Trifft } \\
\text { voll zu } \\
\square\end{array}$ & $\begin{array}{c}\text { Trifft weitgehend } \\
\text { zu } \\
\square\end{array}$ & $\begin{array}{c}\text { Trifft } \\
\text { ziemlich zu } \\
\square\end{array}$ & $\begin{array}{c}\text { Trifft kaum } \\
\text { zu } \\
\square\end{array}$ & $\begin{array}{c}\text { Trifft gar } \\
\text { nicht zu } \\
\square\end{array}$ \\
\hline $\begin{array}{l}\text {...achte ich mehr darauf, } \\
\text { mich nicht lange in der } \\
\text { Sonne aufzuhalten }\end{array}$ & $\begin{array}{c}\text { Trifft } \\
\text { voll zu } \\
\square\end{array}$ & $\begin{array}{c}\text { Trifft weitgehend } \\
\text { zu } \\
\square\end{array}$ & $\begin{array}{c}\text { Trifft } \\
\text { ziemlich zu } \\
\square\end{array}$ & $\begin{array}{c}\text { Trifft kaum } \\
\text { zu } \\
\square\end{array}$ & $\begin{array}{c}\text { Trifft gar } \\
\text { nicht zu } \\
\square\end{array}$ \\
\hline
\end{tabular}

\begin{tabular}{|c|c|c|c|c|c|}
\hline $\begin{array}{l}\text {...benutze ich häufiger als } \\
\text { früher Sonnenschutzcremes } \\
\text { mit Lichtschutzfaktor }\end{array}$ & $\begin{array}{c}\text { Trifft } \\
\text { voll zu } \\
\square\end{array}$ & $\begin{array}{c}\text { Trifft weitgehend } \\
\text { zu } \\
\square\end{array}$ & $\begin{array}{c}\text { Trifft } \\
\text { ziemlich zu } \\
\square\end{array}$ & $\begin{array}{c}\text { Trifft kaum } \\
\text { zu } \\
\square\end{array}$ & $\begin{array}{c}\text { Trifft gar } \\
\text { nicht zu } \\
\square\end{array}$ \\
\hline
\end{tabular}

\begin{tabular}{|l|c|c|c|c|c|}
\hline ...trage ich regelmäßig einen & $\begin{array}{c}\text { Trifft } \\
\text { voll zu }\end{array}$ & $\begin{array}{c}\text { Trifft weitgehend } \\
\text { zu }\end{array}$ & $\begin{array}{c}\text { Trifft } \\
\text { ziemlich zu }\end{array}$ & $\begin{array}{c}\text { Trifft kaum } \\
\text { zu }\end{array}$ & $\begin{array}{c}\text { Trifft gar } \\
\text { nicht zu }\end{array}$ \\
& $\square$ & $\square$ & $\square$ & $\square$ & $\square$ \\
\hline
\end{tabular}

\begin{tabular}{|l|c|c|c|c|c|}
\hline ...trage ich regelmäßig eine & Trifft & Trifft weitgehend & Trifft & Trifft kaum \\
Soll zu & zu & Trifft gar \\
nicht zu \\
\end{tabular}

\begin{tabular}{|l|c|c|c|c|c|}
\hline $\begin{array}{l}\text {...habe ich mein } \\
\text { Urlaubsverhalten geändert } \\
\text { (bespielsweise keinen }\end{array}$ & $\begin{array}{c}\text { Trifft } \\
\text { voll zu }\end{array}$ & $\begin{array}{c}\text { Trifft } \\
\text { weitgehend zu }\end{array}$ & $\begin{array}{c}\text { Trifft ziemlich } \\
\text { zu }\end{array}$ & $\begin{array}{c}\text { Trifft kaum } \\
\text { zu }\end{array}$ & $\begin{array}{c}\text { Trifft gar } \\
\text { nicht zu }\end{array}$ \\
$\begin{array}{l}\text { typischen Strandurlaub } \\
\text { mehr) }\end{array}$ & $\square$ & $\square$ & $\square$ & $\square$ & $\square$ \\
\hline
\end{tabular}

\begin{tabular}{|l|c|c|c|c|c|}
\hline ...vermeide ich es in den & Trifft & Trifft weitgehend & Trifft & Trifft kaum & Trifft gar \\
Mittagsstunden, bei voller & voll zu & zu & nicht zu \\
$\begin{array}{l}\text { Sonneneinstrahlung, das } \\
\text { Haus zu verlassen }\end{array}$ & $\square$ & $\square$ & $\square$ & $\square$ & $\square$ \\
\hline
\end{tabular}

\begin{tabular}{|l|c|c|c|c|c|}
\hline ...habe ich mein & Trifft & Trifft weitgehend & Trifft & Trifft kaum & Trifft gar \\
Freizeitverhalten & voll zu & zu & zuemlich zu & zu \\
(Freibadbesuche, & $\square$ & $\square$ & $\square$ & $\square$ & $\square$ \\
Radtouren) verändert & $\square$ & $\square$ & $\square$ \\
\hline
\end{tabular}

\begin{tabular}{|c|c|c|c|c|c|}
\hline $\begin{array}{l}\text {...ernähre ich mich gesünder } \\
\text { und bewusster }\end{array}$ & $\begin{array}{c}\text { Trifft } \\
\text { voll zu } \\
\square\end{array}$ & $\begin{array}{c}\text { Trifft weitgehend } \\
\text { zu } \\
\square\end{array}$ & $\begin{array}{c}\text { Trifft } \\
\text { ziemlich zu } \\
\square\end{array}$ & $\begin{array}{c}\text { Trifft kaum } \\
\text { zu } \\
\square\end{array}$ & $\begin{array}{c}\text { Trifft gar } \\
\text { nicht zu } \\
\square\end{array}$ \\
\hline ...trinke ich weniger Alkohol & $\begin{array}{c}\text { Trifft } \\
\text { voll zu } \\
\square\end{array}$ & $\begin{array}{c}\text { Trifft weitgehend } \\
\text { zu } \\
\square\end{array}$ & $\begin{array}{c}\text { Trifft } \\
\text { ziemlich zu } \\
\square\end{array}$ & $\begin{array}{c}\text { Trifft kaum } \\
\text { zu } \\
\square\end{array}$ & $\begin{array}{c}\text { Trifft gar } \\
\text { nicht zu } \\
\square\end{array}$ \\
\hline
\end{tabular}


2. Üben oder haben Sie einen Beruf ausgeübt, der vor allem draußen unter Sonneneinstrahlung stattfindet?

$$
\text { Ja } \square \quad \text { Nein } \square
$$

Wenn Sie mit Ja geantwortet haben:

Haben Sie Ihren Beruf nach der Diagnose Ihrer Hautkrebserkrankung gewechselt, bzw werden Sie nun in einem Bereich eingesetzt in dem Sie der Sonneneinstrahlung nicht so sehr ausgesetzt sind?

$$
\text { Ja } \square \quad \text { Nein } \square
$$

3. Sind Sie vor der Diagnose Ihrer Hautkrebserkrankung regelmäßig zur Hautkrebs Vorsorgeuntersuchung gegangen?

Ja

Nein

wenn ja, wie oft?

1 mal pro Jahr $\square \quad$ öfter

4. Gehen Sie nun, nach der Diagnose Ihrer Hautkrebserkrankung regelmäßig zur Vorsorgeuntersuchung?

$$
\text { Ja } \square \quad \text { Nein } \square
$$

wenn ja, wie oft?

1 mal pro Jahr

öfter

5. Rauchen Sie oder haben Sie bis zur Feststellung Ihrer Hautkrebserkrankung geraucht?

Ja $\square \quad$ Nein

Wenn Sie Ja angekreuzt haben:

Wie haben Sie inr Verhalten bezüglich des Rauchens nach bekanntwerden Ihrer Hautkrebserkrankung geändert? Bitte kreuzen Sie die Antwort an die am ehesten auf Sie zutrifft:

$\square$ Ich rauche seit Bekanntwerden meiner Hautkrebserkrankung nicht mehr.

$\square \quad$ Ich habe mein Rauchverhalten seit Bekanntwerden meiner Hautkrebserkrankung deutlich reduziert.

$\square$ Ich habe mein Rauchverhalten nach Bekanntwerden meiner Hautkrebserkrankung nicht verändert.

6. Haben Sie Anmerkungen oder ein Anliegen? Bitte schreiben Sie diese nachfolgend auf: 